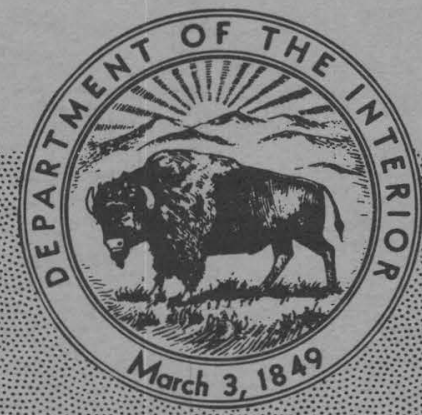

\title{
THE INDUSTRIAL UTILITY OF PUBLIC WATER SUPPLIES IN THE
}

SOUTH ATLANTIC STATES, 1952

\author{
PRDPERTY DF \\ PRDPERTY QF
U. 5. GEDLDGICAL SURVEY,
PUELIE INQUIRIES OFFICE \\ SAN FRANCISFR. CALIFLRNIA
}



UNITED STATES DEPARTMENT OF THE INTERIOR

Douglas McKay, Secretary

GEOLOGICAL SURVEY

W. E. Wrather, Director

GEOLOGICAL SURVEY CIRCULAR 269

\section{THE INDUSTRIAL UTILITY OF PUBLIC WATER SUPPLIES IN THE SOUTH ATLANTIC STATES, 1952}

By E. W. Lohr, F. H. Pauszek, J. G. Connor, W. L. Lamar, and E. F. McCarren 



\section{CONTENTS}

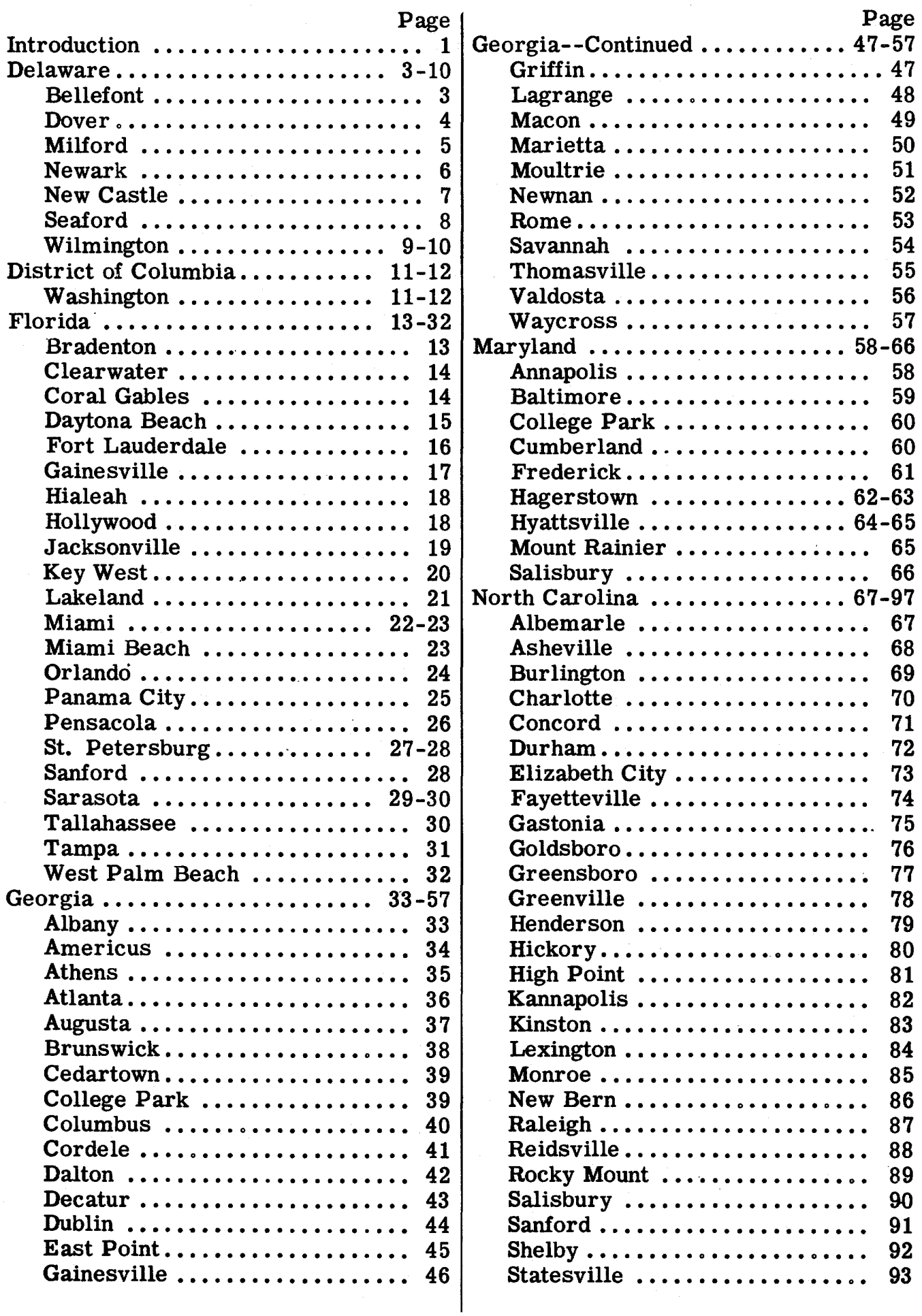




\section{CONTENTS}

\begin{tabular}{|c|c|}
\hline orth Carolina--Continued ..... $\begin{array}{r}\text { Page } \\
94-97\end{array}$ & Virginia--Continued......... 129-147 \\
\hline Thomasville.............. 94 & Danville ................ 129 \\
\hline Wilson $\ldots \ldots \ldots \ldots \ldots \ldots \ldots . \ldots 5$ & Falls Church ....... \\
\hline Wilmington $\ldots \ldots \ldots \ldots \ldots \ldots 96$ & Fredricksburg . . . . . . . . \\
\hline Winston-Salem. . . . . . . . . . 97 & Harrisonburg .......... \\
\hline suth Carolina ............98-124 & Hopewell............. \\
\hline Aiken $\ldots \ldots \ldots \ldots \ldots \ldots \ldots \ldots \ldots 98$ & Lynchburg . . . . . . . . . . 133 \\
\hline Anderson $\ldots \ldots \ldots \ldots \ldots \ldots \ldots 99$ & Martinsville ............. 134 \\
\hline fort ............... 100 & Newport News ........... \\
\hline ettsville............. 101 & olk ............... \\
\hline den $\ldots \ldots \ldots \ldots \ldots \ldots \ldots 102$ & Petersburg .............. \\
\hline Charleston............... 103 & Portsmouth .......... \\
\hline$\ldots \ldots \ldots \ldots \ldots$ & Pulaski ............. \\
\hline$\ldots \ldots \ldots \ldots \ldots \ldots \ldots \ldots 105$ & nond $\ldots \ldots \ldots \ldots$ \\
\hline$\ldots \ldots \ldots \ldots \ldots \ldots$ & Roanoke ......... \\
\hline ................. 107 & Norfolk............. \\
\hline igton $\ldots \ldots \ldots \ldots \ldots \ldots \ldots 108$ & on $\ldots \ldots \ldots \ldots \ldots$ \\
\hline y $\ldots \ldots \ldots \ldots \ldots \ldots \ldots 109$ & Suffolk $\ldots \ldots \ldots \ldots \ldots \ldots \ldots$ \\
\hline Florence .............. 110 & boro .............. \\
\hline ............. 111 & Winchester......... \\
\hline Georgetown $\ldots \ldots \ldots \ldots \ldots \ldots \ldots 112$ & West Virginia $\ldots \ldots \ldots \ldots$ \\
\hline ille $\ldots \ldots \ldots \ldots \ldots \ldots \ldots$ & ............ \\
\hline $\operatorname{wood} \ldots \ldots \ldots \ldots \ldots \ldots \ldots 114$ & ......... \\
\hline $\ldots \ldots \ldots \ldots \ldots \ldots \ldots \ldots 115$ & leston $\ldots \ldots \ldots \ldots \ldots \ldots \ldots 150$ \\
\hline ille $\ldots \ldots \ldots \ldots \ldots \ldots \ldots 116$ & Clarksburg.$\ldots \ldots \ldots \ldots \ldots$ \\
\hline$s \ldots \ldots \ldots \ldots \ldots \ldots \ldots 117$ & Elkins $\ldots \ldots \ldots \ldots \ldots \ldots$ \\
\hline$\ldots \ldots \ldots \ldots \ldots \ldots \ldots \ldots 118$ & Fair \\
\hline$y \ldots \ldots \ldots$ & Hunt \\
\hline ngeburg $\ldots \ldots \ldots \ldots$ & Martinsburg.... \\
\hline ....... & ntown $\ldots \ldots$ \\
\hline lburg . . . . . . & dsville ... \\
\hline Sumter $\ldots \ldots \ldots \ldots \ldots \ldots \ldots \ldots \ldots 123$ & Nitro $\ldots \ldots \ldots \ldots \ldots \ldots \ldots \ldots$ \\
\hline$\ldots \ldots \ldots \ldots \ldots \ldots \ldots \ldots 124$ & rkersburg $\ldots \ldots \ldots \ldots \ldots$ \\
\hline $\ldots \ldots \ldots \ldots \ldots 125-147$ & $\ldots 160$ \\
\hline & South \\
\hline ...... & ... 161 \\
\hline Charlottesville & \\
\hline & \\
\hline
\end{tabular}

\section{ILLUSTRATION}

Figure 1. Map of the United States showing sections covered by the nine circulars on the Industrial Utility of Public Water Supplies of the United States, 1952. The shaded portion represents the section of the country covered by this cir-

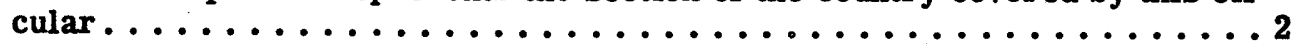




\title{
THE INDUSTRIAL UTILITY OF PUBLIC WATER SUPPLIES IN THE SOUTH ATLANTIC STATES, 1952
}

\author{
By E. W. Lohr, F. H. Pauszek, J. G. Connor, \\ W. L. Lamar, and E. F. McCarren
}

\section{INTRODUCTION}

The location of industrial plants is dependent on an ample water supply of suitable quality. Information relating to the chemical characteristics of the water supplies is not only essential to the location of many plants but also is an aid in the manufacture and distribution of many commodities.

Public water supplies are utilized extensively as a source of supply for many industrial plants, used either as delivered for domestic consumption or with further treatment if necessary to meet specific needs of the plant, such as water for processing, cooling, and steam generation. The industrial use of water in the United States in 1950 was estimated to be more than $\mathbf{7 5}$ billion gallons per day from private sources. In addition, about 6 billion gallons per day was estimated to be taken from public water supplies.

U. S. Geological Survey Water-Supply Paper 658, "The industrial utility of public water supplies in the United States, 1932" contains information pertaining to the public water supplies of 670 of the larger cities throughout the United States. This report, which is still in print and being distributed, has filled an important need in the field of water-supply engineering. The demand for more up-to-date information and more extended coverage has led to studies by the Geological Survey for revision of the information contained in the 1932 report. The revised report, which will include data pertaining to public water supplies of more than 1,200 cities in the United States, will eventually be published as a Geological Survey Water-Supply Paper. However, in order that the information might be available at the earliest possible time, nine preliminary reports are being issued which give data on the larger cities in each state. These nine reports are being released as Geological Survey Circulars, each covering a group of states as delineated by the Bureau of Census in taking the census of the population of the country. (See fig. 1). The reports give descriptive information and analytical data for approximately three-fourths of the cities that will be included in the final report for each of the states.

This circular is the seventh of the series and includes data for the States of Delaware, Florida, Georgia, Maryland, North Carolina, South Carolina, Virginia, and West Virginia, and the District of Columbia. (See fig. 1). The report gives the population (1950) of the city, the population supplied, ownership, sources and treatment of supplies, storage facilities for both raw and finished waters, and chemical analyses of the water for a total of 162 of the larger cities in the states of the South Atlantic group. The data for each city are essentially the same as will appear in the complete report for the whole country.

Data for the supplies in Delaware were compiled by E. F. McCarren, chemist,

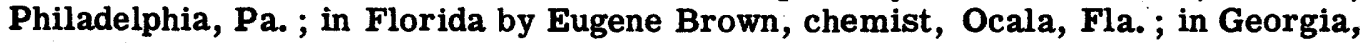
North Carolina, and South Carolina by F. H. Pauszek, district chemist, Raleigh, N. C. ; in Maryland and the District of Columbia by E. W. Lohr; in Virginia by J. G. Connor, district chemist, Charlottesville, Va. ; and in West Virginia by P. N. Brown, chemist, under the supervision of W. L. Lamar, district chemist, Columbus, Ohio. Review and final assembly of the data were made by E. W. Lohr in the Washington office under the direction of $\mathrm{S}$. $\mathrm{K}$. Love, Chief, Quality of Water Branch. 


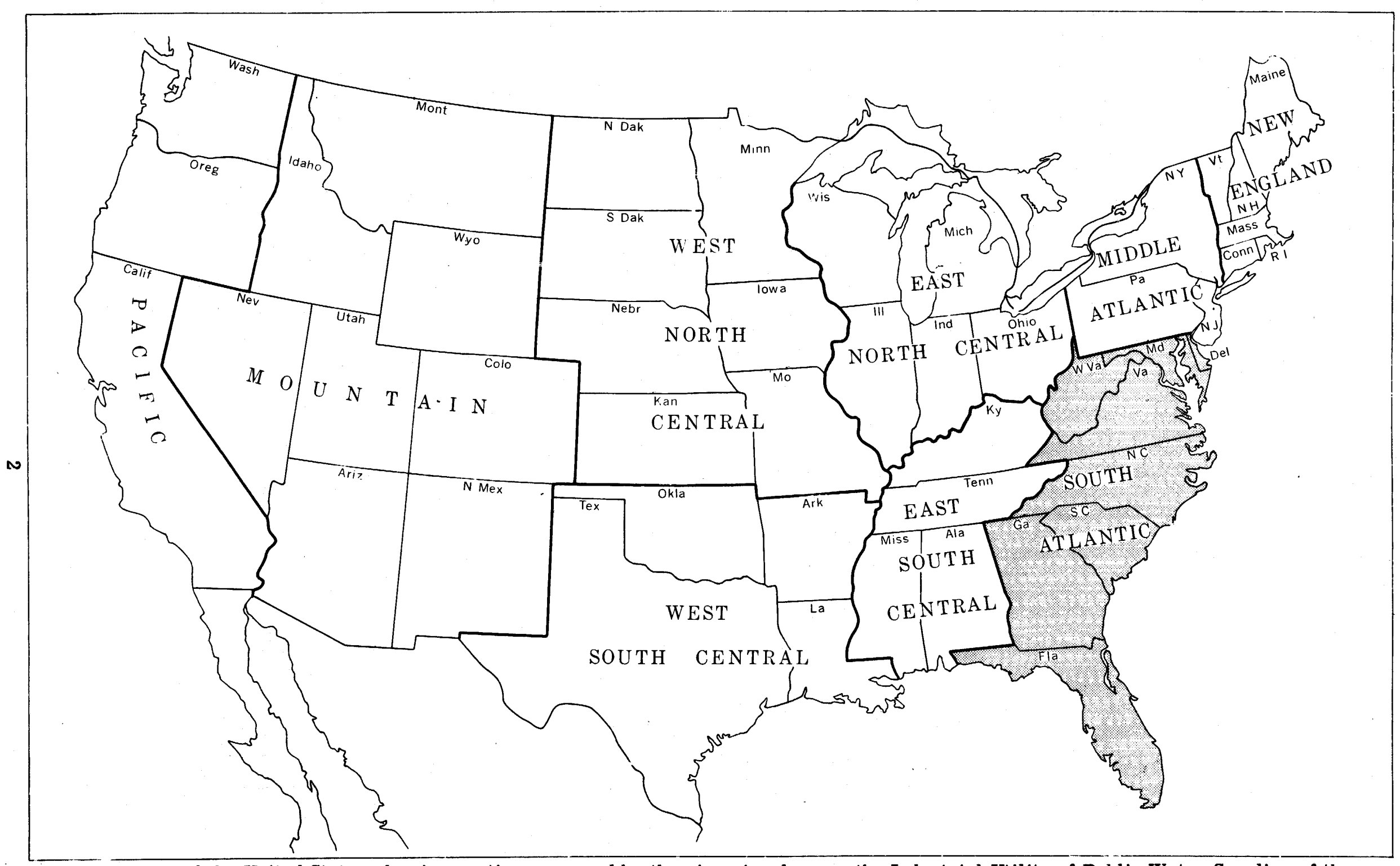

Figure 1. -Map of the United States showing sections covered by the nine circulars on the Industrial Utility of Public Water Supplies of the United States, 1952. The shaded portion represents the section of the country covered by this circular. 


\section{BELLEFONT}

(Population, 1, 472)

Ownership: Wilmington Suburban Water Co. ; supplies also Bellevue, Claymont, Gwinhurst, Holly Oak, Silverside, and other communities in New Castle County. Total population supplied, about 18,000.

Source: Bellevue Quarry (largely spring fed) about 75 percent of the supply. The remaining 25 percent of the supply is furnished by the Delaware Water Co. and Chester Municipal Authority.

Treatment: Wilmington Suburban Water Co. supply: prechlorination, coagulation with alum, sedimentation, rapid sand filtration, postchlorination, and adjustment of $\mathrm{pH}$ with lime.

Rated capacity of treatment plant: $1,440,000$ gpd.

Raw-water storage: 100,000,000 gal. (Bellevue quarry).

Finished-water storage: 600,000 gal.

The treatment plant is at Bellevue.

\section{ANALYSES}

(Analyses, in parts per million, by U. S. Geological Survey)

\begin{tabular}{|c|c|c|c|c|c|}
\hline & $\begin{array}{c}\text { Raw } \\
\text { water a }\end{array}$ & $\begin{array}{c}\text { Finished } \\
\text { water } \\
\text { (city tap) }\end{array}$ & & $\begin{array}{c}\text { Raw } \\
\text { water a }\end{array}$ & $\begin{array}{l}\text { Finished } \\
\text { water } \\
\text { (city tap) }\end{array}$ \\
\hline 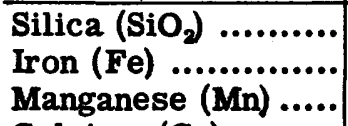 & $\begin{array}{l}-- \\
--\end{array}$ & \begin{tabular}{|c|}
9.9 \\
.24 \\
--
\end{tabular} & $\begin{array}{r}\text { Hardness as } \mathrm{CaCO}_{3}: \\
\text { Total } . . . . . . . . . . . . . . . . \\
\text { Noncarbonate...... }\end{array}$ & $\begin{array}{r}22 \\
9\end{array}$ & $\begin{array}{l}70 \\
25 \\
\end{array}$ \\
\hline 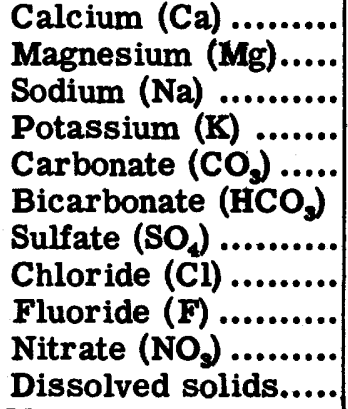 & $\begin{array}{c}-- \\
-- \\
0 \\
0 \\
16 \\
20 \\
6.0 \\
1.4 \\
--\end{array}$ & $\begin{array}{c}22 \\
3.8 \\
5.3 \\
2.2 \\
0 \\
55 \\
24 \\
12 \\
.2 \\
1.4 \\
122\end{array}$ & 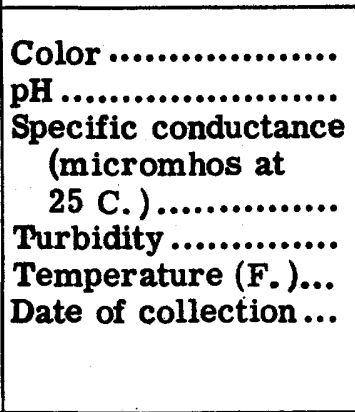 & $\begin{array}{r}103 \\
-- \\
50 \\
\text { May 24, } \\
1951\end{array}$ & $\begin{array}{r}197 \\
54 \\
54 \\
\text { May } 24, \\
1951\end{array}$ \\
\hline
\end{tabular}

a Bellevue Quarry. 


\section{DOVER \\ (Population, 6, 223)}

Ownership: Municipal; also supplies about 500 people outside the city limits.

Total population supplied, about 6,700 .

Source: 3 deep wells (1 to 3) 208, 221 , and $222 \mathrm{ft}$ deep; 1 ("A") open well. The deep wells are at the power plant, on Division Street, and on Dover Street, respectively, and are reported to yield 505, 650, and $657 \mathrm{gpm}$.

Treatment: None.

Storage: Tank, 100,000 gal.

\section{ANALYSES}

(Analyses, in parts per million, by U. S. Geological Survey)

\begin{tabular}{|c|c|c|c|c|c|}
\hline & $\begin{array}{l}\text { Well 1, } \\
\text { Power } \\
\text { Plant }\end{array}$ & $\begin{array}{c}\text { Well 2, } \\
\text { Division } \\
\text { Street }\end{array}$ & $\begin{array}{l}\text { Well 3, } \\
\text { Dover St. }\end{array}$ & $\begin{array}{c}\text { Well "A", } \\
\text { Open } \\
\text { well }\end{array}$ & $\begin{array}{l}\text { City tap } \\
\text { sample }\end{array}$ \\
\hline 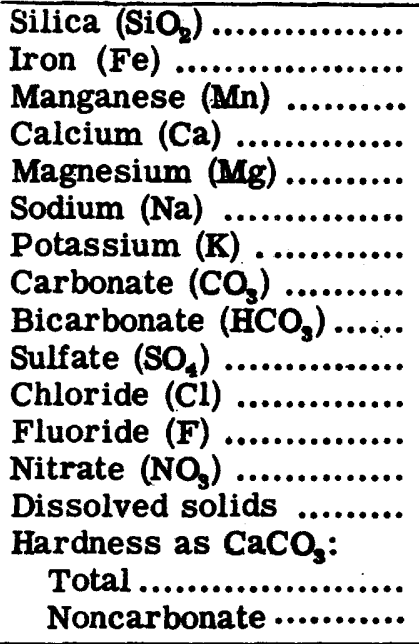 & $\begin{array}{r}-- \\
-- \\
-- \\
-- \\
-- \\
-- \\
0 \\
0 \\
157 \\
6.6 \\
3.4 \\
-- \\
.4 \\
--\end{array}$ & $\begin{array}{r}-- \\
-- \\
-- \\
0 \\
0 \\
0 \\
153 \\
8.9 \\
3.4 \\
-- \\
.5 \\
--\end{array}$ & $\begin{array}{r}-- \\
-- \\
-- \\
-- \\
-- \\
0 \\
0 \\
153 \\
7.5 \\
2.8 \\
-- \\
.4 \\
--\end{array}$ & $\begin{array}{r}-- \\
--- \\
-- \\
-- \\
-- \\
0 \\
0 \\
153 \\
7.8 \\
3.4 \\
--5 \\
--\end{array}$ & $\begin{array}{c}35 \\
.08 \\
33 \\
5.0 \\
14 \\
2.9 \\
0 \\
153 \\
8.0 \\
3.0 \\
.1 \\
.6 \\
191\end{array}$ \\
\hline $\begin{array}{l}\text { Color ........................ } \\
\text { pH .............................. } \\
\text { Specific conductance } \\
\quad \text { (micromhos at } 25 \text { C. }) \\
\text { Turbidity ................... } \\
\text { Temperature }(\mathrm{F} . \text { )......... } \\
\text { Date of colection ........ }\end{array}$ & $\begin{array}{r}5 \\
8.0 \\
259 \\
-- \\
60 \\
\text { Apr. 25, } \\
1951\end{array}$ & $\begin{array}{r}5 \\
8.1 \\
\\
254 \\
-- \\
58 \\
\text { Apr. } 25, \\
1951 \\
\end{array}$ & $\begin{array}{r}8 \\
8.0 \\
252 \\
-- \\
61 \\
\text { Apr. 25, } \\
1951 \\
\end{array}$ & $\begin{array}{r}5.0 \\
261 \\
-- \\
68 \\
\text { Apr. } 25, \\
1951 \\
\end{array}$ & $\begin{array}{r}7 \\
8.0 \\
263 \\
-- \\
64 \\
\text { Apr. } 25, \\
1951 \\
\end{array}$ \\
\hline $\begin{array}{l}\text { Depth (feet) } \ldots . . . \ldots . . . \ldots . . \\
\text { Diameter (inches)......... } \\
\text { Date drilled ............... } \\
\text { Percent of supply ........ }\end{array}$ & $\begin{array}{r}208 \\
12 \\
1926 \\
--\end{array}$ & $\begin{array}{r}231 \\
12 \\
1938 \\
--\end{array}$ & $\begin{array}{r}222 \\
16-10 \\
1948 \\
--\end{array}$ & $\begin{array}{l}-- \\
-- \\
--\end{array}$ & $\begin{array}{l}-- \\
-- \\
--\end{array}$ \\
\hline
\end{tabular}




\section{MILFORD}

(Population, 5, 179)

Ownership: Municipal.

Source: 3 deep wells ( 1 to 3 ) 150,150 , and $220 \mathrm{ft}$ deep. Well 3 is reported to yield 346 gpm.

Treatment: Aeration and chlorination.

Rated capacity of treatment plant: $500,000 \mathrm{gpd}$.

Raw-water storage: None.

Finished-water storage: 250,000 gal.

\section{ANALYSES}

(Analyses, in parts per million, by U. S. Geological Survey)

\begin{tabular}{|c|c|c|c|}
\hline & Well 1 & Well 3 & $\begin{array}{c}\text { Finished } \\
\text { water a }\end{array}$ \\
\hline 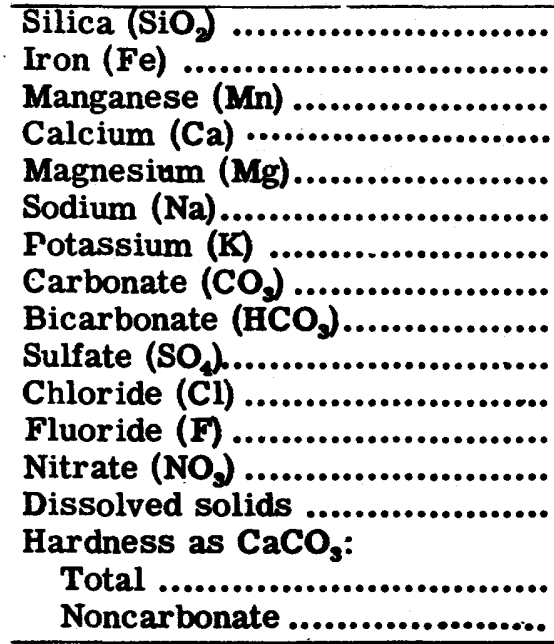 & $\begin{array}{r}-- \\
-- \\
-- \\
-- \\
-- \\
0 \\
189 \\
2.4 \\
2.8 \\
.- \\
.- \\
140 \\
0\end{array}$ & $\begin{array}{c}55 \\
.13 \\
.01 \\
48 \\
6.9 \\
6.4 \\
2.5 \\
0 \\
192 \\
2.0 \\
3.4 \\
.1 \\
.0 \\
217 \\
148 \\
0\end{array}$ & $\begin{array}{c}36 \\
.27 \\
46 \\
6.6 \\
5.6 \\
2.9 \\
0 \\
187 \\
2.9 \\
2.9 \\
.0 \\
.3 \\
216 \\
142 \\
0 \\
\end{array}$ \\
\hline 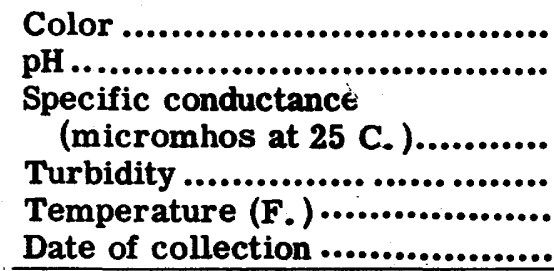 & $\begin{array}{r}2 \\
7.9 \\
298 \\
-- \\
60 \\
\text { Apr. } 20,1951 \\
\end{array}$ & $\begin{array}{r}2 \\
7.9 \\
296 \\
-- \\
\text { Dec. } 12,195 \overline{-} \\
\end{array}$ & \begin{tabular}{|r|}
4 \\
8.1 \\
301 \\
-- \\
57 \\
Apr. 20, 1951
\end{tabular} \\
\hline
\end{tabular}

a Well 2 out of operation at time of sampling. 
NEWARK

(Population, 6, 731)

Ownership: Municipal。

Source: 4 wells $(1,4,5,7) 72,62,70$, and $64 \mathrm{ft}$ deep; yield reported to be 146 , 160,200 , and $300 \mathrm{gpm}$.

Treatment: Chlorination.

Rated capacity of treatment plant: $1,296,000$ gpd.

Raw-water storage: 150,000 gal.

Finished-water storage: 752,000 gal.

ANALYSES

(Analyses, in parts per million, by U. S. Geological Survey)

\begin{tabular}{|c|c|c|c|c|c|}
\hline & Well 1 & Well 4 & Well 5 & Well 7 & $\begin{array}{l}\text { Finished } \\
\text { water } \\
\text { (city tap) }\end{array}$ \\
\hline 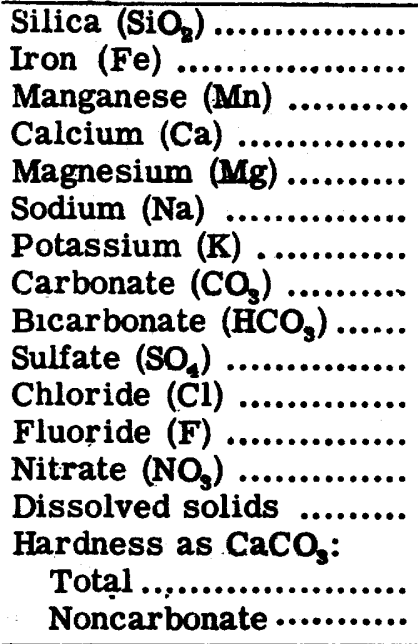 & $\begin{array}{l}-- \\
-- \\
-- \\
-- \\
0 \\
9 \\
5.8 \\
12 \\
19 \\
\\
\\
30 \\
23\end{array}$ & 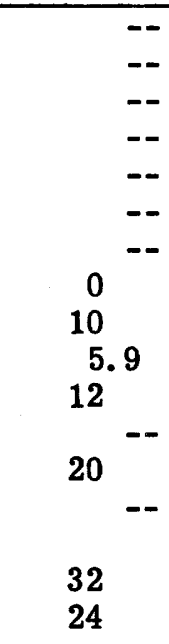 & 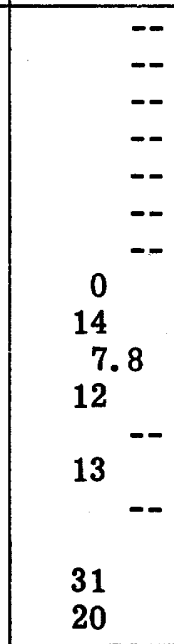 & 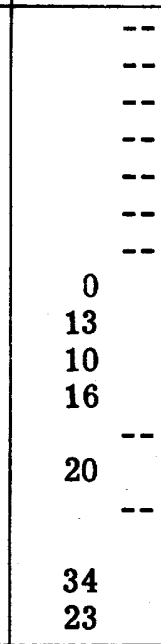 & $\begin{array}{c}13 \\
.61 \\
-{ }^{-} \\
6.8 \\
4.3 \\
7.6 \\
1.1 \\
0 \\
11 \\
8.2 \\
15 \\
16 \\
16 \\
95\end{array}$ \\
\hline 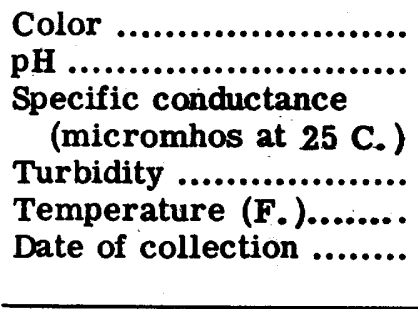 & $\begin{array}{r}5 \\
5.9 \\
114 \\
-- \\
56 \\
\text { Apr. } 26, \\
1951\end{array}$ & $\begin{array}{r}5 \\
5.8 \\
\\
113 \\
-- \\
58 \\
\text { Apr. } 26, \\
1951\end{array}$ & $\begin{array}{r}5 \\
6.5 \\
111 \\
-- \\
58 \\
\text { Apr. 26, } \\
1951\end{array}$ & $\begin{array}{r}5 \\
6.0 \\
144 \\
- \\
56 \\
\text { Apr. 26, } \\
1951\end{array}$ & $\begin{array}{r}3 \\
6.8 \\
136 \\
- \\
57 \\
\text { Apr. 26, } \\
1951\end{array}$ \\
\hline $\begin{array}{l}\text { Depth (feet) } \ldots . . . . . . . . . . . . \\
\text { Diameter (inches)......... } \\
\text { Date drilled ............... } \\
\text { Percent of supply ........ }\end{array}$ & $\begin{array}{r}72 \\
6 \\
1906 \\
--\end{array}$ & $\begin{array}{r}62 \\
10 \\
1920 \\
--\end{array}$ & $\begin{array}{r}70 \\
10 \\
1920 \\
--\end{array}$ & $\begin{array}{r}64 \\
16 \\
1931 \\
--\end{array}$ & \\
\hline
\end{tabular}




\section{NEW CASTLE \\ (Population, 5, 396)}

Ownership: Municipal.

Source: 3 interconnected wells (Cd1, Cd2, Cd3) each $24 \mathrm{ft}$ deep and 3 infiltration galleries which drain into the wells. Water from well Cd1 flows by gravity through an infiltration gallery to well Cd2; water from well Cd2 flows by gravity to well $\mathrm{Cd} 3$, which also receives the combined flow of water from the other two infiltration galleries. Wells $\mathrm{Cd} 1$ and $\mathrm{Cd} 2$ furnish 50 percent of the supply; well Cd3 with the combined flow of the 2 galleries furnish 50 percent of the supply.

Treatment: Aeration, coagulation with alum, sedimentation, rapid (pressure) filtration, and chlorination.

Rated capacity of treatment plant: $1,000,000$ gpd.

Raw-water storage: 645,000 gal.

Finished-water storage: $1,500,000 \mathrm{gal}$.

\section{ANALYSES}

(Analyses, in parts per million, by U. S. Geological Survey)

\begin{tabular}{|c|c|c|c|c|}
\hline & Well Cd1 & Well Cd2 & $\begin{array}{c}\text { Infiltration } \\
\text { galleries }\end{array}$ & $\begin{array}{c}\text { Finished } \\
\text { water }\end{array}$ \\
\hline 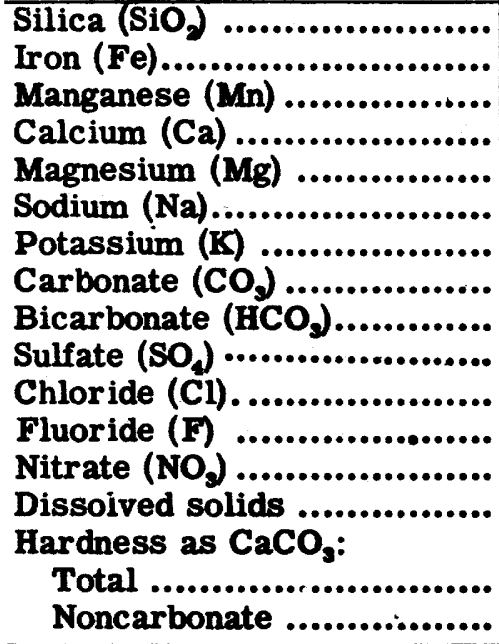 & 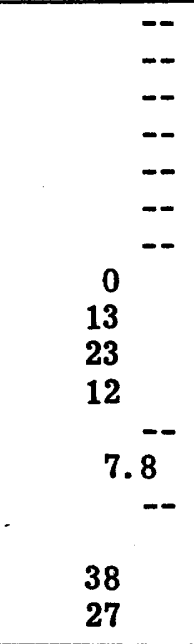 & $\begin{array}{l}-- \\
-- \\
-- \\
-- \\
-- \\
0 \\
-- \\
12 \\
16 \\
9.8 \\
8.8 \\
\\
-- \\
29 \\
19\end{array}$ & $\begin{array}{r}-- \\
-- \\
-- \\
-- \\
0 \\
03 \\
100 \\
240 \\
1.6 \\
1 .- \\
161 \\
126\end{array}$ & $\begin{array}{l}16 \\
.40 \\
28 \\
18 \\
80 \\
3.9 \\
0 \\
52 \\
49 \\
162 \\
.0 \\
4.1 \\
445 \\
144 \\
101\end{array}$ \\
\hline 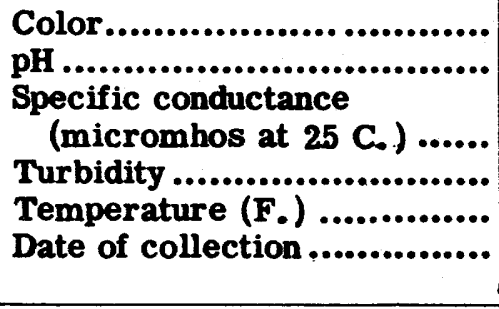 & $\begin{array}{r}5 \\
6.2 \\
138 \\
-- \\
57 \\
\text { Apr. } 26, \\
1951 \\
\end{array}$ & $\begin{array}{r}5 \\
6.3 \\
114 \\
-- \\
56 \\
\text { Apr. } 26, \\
1951\end{array}$ & $\begin{array}{r}10 \\
6.5 \\
1,000 \\
-\overline{58} \\
\text { Apr. } 26, \\
1951\end{array}$ & $\begin{array}{r}3 \\
7.7 \\
716 \\
-- \\
56 \\
\text { Apr. 26, } \\
1951\end{array}$ \\
\hline 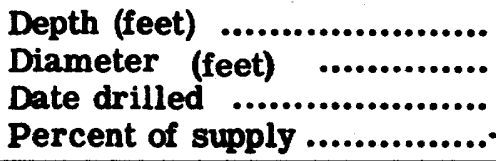 & $\begin{array}{l}24 \\
12 \\
-- \\
--\end{array}$ & $\begin{array}{l}24 \\
12 \\
-- \\
--\end{array}$ & & \\
\hline
\end{tabular}

a Sample from the combined flow from the two galleries flowing into well Cd3. 


\section{SEAFORD}

(Population, 3, 087)

Ownership: Municipal.

Source: 8 wells: 5 wells, each $80 \mathrm{ft}$ deep; 2 wells, each $40 \mathrm{ft}$ deep; 1 well, 73 $\mathrm{ft}$ deep. The wells are pumped in groups of 5,3 , and 1 wells by 3 pumping units, Standby, Power Plant, and Spruce St., respectively.

Treatment: Adjustment of $\mathrm{pH}$ with lime.

Rated capacity of treatment plant: 2,016,000 gpd.

Raw-water storage: None.

Finished-water storage: 300,000 gal.

\section{ANALYSES}

(Analyses, in parts per million, by U. S. Geological Survey)

\begin{tabular}{|c|c|c|c|c|}
\hline & 5 wells & 2 wells & $\begin{array}{c}1 \text { well } \\
\text { Spruce St. }\end{array}$ & $\begin{array}{l}\text { Finished } \\
\text { water } \\
\text { (city tap) }\end{array}$ \\
\hline Silica $\left(\mathrm{SiO}_{2}\right) \ldots \ldots \ldots \ldots \ldots \ldots \ldots$ & -- & -- & -- & 14 \\
\hline Iron $(\mathrm{Fe}) \ldots \ldots \ldots \ldots \ldots \ldots \ldots \ldots . . . . . . .$. & -- & -- & -- & .07 \\
\hline Manganese (Mn) ................. & -- & -- & -- & -- \\
\hline Calcium (Ca) ..................... & -- & -- & -- & 5.3 \\
\hline Magnesium (Mg) ................ & -- & -- & -- & 3.0 \\
\hline Sodium (Na)....................... & -- & -- & -- & 5.9 \\
\hline Potassium (K) .................. & -- & -- & -- & 2.6 \\
\hline Carbonate $\left(\mathrm{CO}_{3}\right) \ldots \ldots \ldots \ldots \ldots$ & & 0 & 0 & 0 \\
\hline Bicarbonate $\left(\mathrm{HCO} \mathrm{O}_{3}\right) . . . . . . . . . .$. & 7 & 20 & 5 & 6 \\
\hline Sulfate $\left(\mathrm{SO}_{4}\right) \ldots \ldots \ldots \ldots \ldots \ldots \ldots$ & 7.8 & 8.7 & 5.1 & 7.7 \\
\hline Chloride (Cl) .................... & 9.8 & 11 & 8.2 & 9.0 \\
\hline Fluoride $(\boldsymbol{F})$ & & -- & -- & .1 \\
\hline Nitrate $\left(\mathrm{NO}_{3}\right) \ldots \ldots \ldots \ldots \ldots \ldots \ldots$ & 23 & 23 & 19 & 22 \\
\hline Dissolved solids ................ & -- & -- & & 89 \\
\hline $\begin{array}{l}\text { Hardness as } \mathrm{CaCO}_{\mathbf{3}}: \\
\text { Total }\end{array}$ & 23 & 26 & 20 & 24 \\
\hline Noncarbonate .................. & 17 & 9.6 & 16 & 21 \\
\hline Color........................... & 4 & 2 & 2 & \\
\hline pH $\ldots \ldots \ldots \ldots \ldots \ldots \ldots \ldots \ldots$ & 5.8 & 6.2 & 5.4 & 5.5 \\
\hline $\begin{array}{l}\text { Specific conductance } \\
\text { (micromhos at } 25 \mathrm{C} \text { ) }\end{array}$ & 114 & 124 & 961 & 109 \\
\hline Turbidity...................... & 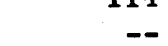 & -- & - & 100 \\
\hline Temperature (F.) ............... & 59 & 58 & 57 & 68 \\
\hline Date of collection ................. & $\begin{array}{l}\text { Apr. } 20 \\
1951\end{array}$ & $\begin{array}{l}\text { Apr. } 20, \\
1951\end{array}$ & $\begin{array}{l}\text { Apr. } 20, \\
1951\end{array}$ & $\begin{array}{l}\text { Apr. } 20, \\
1951\end{array}$ \\
\hline
\end{tabular}

Regular determinations at treatment plant, 1950

\begin{tabular}{l|r|r|r|r|r|r|r|r|r|r|r|r}
\hline & \multicolumn{3}{|c|}{$\begin{array}{c}\text { Alkalinity } \\
\text { as CaCO } \\
\text { (ppm) }\end{array}$} & \multicolumn{3}{c|}{ pH } & \multicolumn{3}{c|}{$\begin{array}{c}\text { Hardness } \\
\text { as CaCO } \\
\text { (ppm) }\end{array}$} & \multicolumn{3}{c}{ Turbidity } \\
\cline { 2 - 22 } & Av & Max & Min & Av & Max & Min & Av & Max & Min & Av & Max & Min \\
\hline Raw water........ & 6 & 7 & 5 & 5.7 & -- & -- & 34 & 38 & 32 & 0 & -- & -- \\
Finished water... & -- & -- & -- & 7.0 & -- & -- & -- & -- & -- & -- & -- & - \\
\hline
\end{tabular}




\section{WILMINGTON}

(Population, 110, 356)

Ownership: Municipal; supplies also about 13,500 people outside the city limits. Total population supplied, about 123,900 .

Source: Brandywine Creek.

Treatment: Porter plant: sedimentation, slow sand filtration, and chlorination. Walnut St. plant: Coagulation with alum and lime, sedimentation, rapid sand filtration, and chlorination.

Rated capacity of treatment plants: Porter plant, 12,000,000 gpd; Walnut St. plant, 20,000,000 gpd.

Raw-water storage: Hoopes Reservoir, 2, 235,000,000 gal.

Finished-water storage: Cool Spring Reservoir, 40,000,000 gal; Rodney St. Reservoir, 7,00C,000 gal; clear well, 6, 000,000 gal; Rockford Tower, 500, 000 gal; Monroe Park tank and Faulk Road tank, each 100,000 gal.

In normal operation, water from Brandywine Creek is pumped to Hoopes Reservoir, which serves as a reserve supply in the event of periods of low flow in the Creek. Water is also taken directly from the Creek to the rapid sand filter plant, from which point a portion of the raw water is lifted to the Porter sedimentation reservoir which serves the Porter slow sand filter plant. The finished water from the Porter treatment plant is used in the high service lines. The Walnut Street piant supplies the low service lines.

\section{ANALYSES}

(Analyses, in parts per million, by U. S. Geological Survey)

\begin{tabular}{|c|c|c|c|}
\hline & \multicolumn{2}{|c|}{ Brandywine Creek } & \multirow{2}{*}{$\begin{array}{l}\text { Finished } \\
\text { water } \\
\text { (city tap) }\end{array}$} \\
\hline & Raw water a & Raw water & \\
\hline Silica $\left(\mathrm{SiO}_{2}\right) \ldots \ldots \ldots \ldots \ldots \ldots \ldots \ldots$ & 12 & 12 & 12 \\
\hline Iron $(\mathrm{Fe})$ & .08 & .08 & .10 \\
\hline Manganese (Mn) ..................... & & -- & -- \\
\hline Calcium (Ca) $\ldots . . . \ldots \ldots \ldots \ldots \ldots . . . . . . . .$. & 14 & 11 & 12 \\
\hline Magnesium (Mg).................... & 5.2 & 4.5 & 4.4 \\
\hline Sodium $(\mathrm{Na}), \ldots . . . \ldots \ldots \ldots \ldots \ldots . . . \ldots \ldots$ & -- & -- & 5.4 \\
\hline Fotassium $(\mathbf{K})$...................... & -- & -- & 1.0 \\
\hline Carbonate $\left(\mathrm{CO}_{3}\right)$...................... & 0 & & 0 \\
\hline Bicarbonate $\left(\mathrm{HCO}_{3}\right) \ldots \ldots \ldots \ldots \ldots$ & 41 & 38 & 27 \\
\hline Sulfate $\left(\mathrm{SO}_{4}\right) \ldots \ldots \ldots \ldots \ldots \ldots \ldots$ & 22 & 17 & 28 \\
\hline Chloride $(\mathrm{Cl}) \ldots \ldots \ldots \ldots \ldots \ldots \ldots$ & 6.5 & 4.8 & 6.0 \\
\hline Fluoride $(F) \ldots \ldots \ldots \ldots \ldots \ldots$ & .1 & .1 & .0 \\
\hline Nitrate $\left(\mathrm{NO}_{3}\right)$......................... & 5.7 & 5.7 & 3.9 \\
\hline Dissolved solids .................... & 103 & 89 & 89 \\
\hline Hardness as $\mathrm{CaCO}_{3}:$ & & & \\
\hline Total $\ldots \ldots \ldots \ldots \ldots \ldots \ldots \ldots$ & 56 & 46 & 48 \\
\hline Noncarbonate ...................... & 23 & 15 & 26 \\
\hline Color .................................... & 20 & 5 & 3 \\
\hline 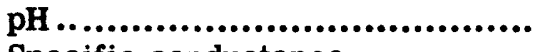 & 6.5 & 6.8 & 7.1 \\
\hline Specific conductance & & & \\
\hline 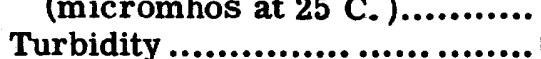 & 158 & 131 & 137 \\
\hline 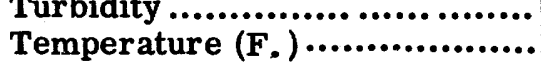 & $\overline{36}$ & $\overline{64}$ & $\overline{60}$ \\
\hline Date of collection..........$\ldots \ldots \ldots$ & $\begin{array}{c}\text { Oct: } 1948, \\
\text { Apr. } 1951\end{array}$ & $\begin{array}{c}\text { May 31 } \\
1950\end{array}$ & $\begin{array}{c}\text { Apr. 25, } \\
1951\end{array}$ \\
\hline
\end{tabular}

a Average of analyses of $\mathbf{3 0}$ monthly samples. 
DELAWARE

WILMINGTON--Continued

Regular determinations at treatment plant, 1950

\begin{tabular}{l|r|r|r|r|r|r|r|r|r|r|r|r}
\hline & \multicolumn{3}{|c|}{$\begin{array}{c}\text { Alkalinity } \\
\text { as CaCO, } \\
\text { (ppm) }\end{array}$} & \multicolumn{3}{c|}{ pH } & \multicolumn{3}{c|}{$\begin{array}{c}\text { Hardness } \\
\text { as CaCO } \\
\text { (ppm) }\end{array}$} & \multicolumn{3}{c}{ Turbidity } \\
\cline { 2 - 13 } & Av & Max & Min & Av & Max & Min & Av & Max & Min & Av & Max & Min \\
\hline Raw water.......... & 35 & 49 & 10 & 7.2 & 8.4 & 6.9 & -- & -- & -- & 40 & 800 & 8 \\
Finished water... & 27 & 40 & 3 & 6.5 & 6.9 & 5.3 & 52 & 62 & 40 & 0 & 0 & 0 \\
\hline
\end{tabular}




\author{
WASHINGTON \\ (Population, 802, 178)
}

Ownership: Department of the Army and the District of Columbia; also supplies Arlington County, Virginia; part of Fairfax County, Virginia; the City of Falls Church, Virginia; and a small area in Montgomery County, Maryland. Total population supplied, about $1,000,000$.

Source: Potomac River. Emergency supply, connections to the extent of $9.2 \mathrm{mgd}$ to the treated supply of the Washington Suburban Sanitary District. (See Hyattsville, Maryland.)

Treatment: Dalecarlia Plant: Prechlorination, coagulation with alum, sedimentation, rapid sand filtration, postchlorination or dechlorination as may be necessary, chlorine dioxide when necessary for control of tastes and odors, and addition of lime for adjustment of pH. McMillan Plant: Addition of chlorine and alum at the Dalecarlia Plant. The water then flows by gravity to the Georgetown Reservoir, 2 miles to the southeast, part of which is constructed to serve as a sedimentation basin. The water then flows by gravity through the Washington Tunnel to the McMillan Reservoir near the center of the District where further settling takes place; and slow sand filtration. The remainder of the treatment is the same as at the Dalecarlia Plant.

Rated capacity of treatment plants: Dalecarlia Plant, 100, 000,000 gpd; McMillan Plant, 125, 000, 000 gpd.

Raw-water storage: $560,000,000$ gal (30 percent available). The three reservoirs, Dalecarlia, Georgetown, and McMillan serve as storage reservoirs for unfiltered water.

Finished-water storage: Clear-water basins, 47, 700,000 gal; ground-surface reservoirs, 67,200,000 gal; elevated tanks, 2, 740,000 gal.

The water system of the District has two components, the supply division and the distribution system. The supply division comprised of the collection and purification systems is under the control of the Department of the Army and is operated by the Washington District Office of the Corps of Engineers. The distribution system is owned and operated by the District of Columbia.

The diversion dam and the raw-water intake are located at Great Falls, Montgomery County, Maryland, about 10 miles from the District line. The raw water flows by gravity through two conduits into the forebay of the Dalecarlia Reservoir at the District line, from which it is lifted into Dalecarlia Reservoir. This reservoir serves not only as storage reservoir but also as a plain sedimentation basin, from which the water flows by gravity to the treatment plants.

Arlington County, Falls Church, and areas in Fairfax County are served principally with water from the Dalecarlia Plant. 
WASHINGTON--Continued

\section{ANALYSES}

(Analyses, in parts per million, by Dalecarlia Laboratory, Washington, D. C.)

\begin{tabular}{|c|c|c|c|c|}
\hline & \multirow{2}{*}{$\begin{array}{c}\text { Raw } \\
\text { water a }\end{array}$} & \multicolumn{2}{|c|}{ Finished water b } & \multirow{2}{*}{$\begin{array}{l}\text { Finished } \\
\text { water c } \\
\text { (city tap) }\end{array}$} \\
\hline & & $\begin{array}{l}\text { Dalecarlia } \\
\text { Plant }\end{array}$ & $\begin{array}{l}\text { McMillan } \\
\text { Plant }\end{array}$ & \\
\hline Silica (SiO $)$...................... & 5.5 & 5.6 & 5.5 & 6.6 \\
\hline 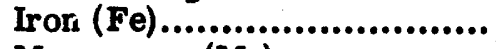 & .05 & .05 & .03 & .00 \\
\hline Manganese $(\mathbf{M n})$.................. & -- & & & \\
\hline Calcium (Ca) ...................... & 25 & 30 & 28 & 31 \\
\hline Sodium $(\mathrm{Na}) . . . . . . . . . . . . .$. & & & 0.4 & \\
\hline Potassium (K) ..... & 6.1 & 4.0 & 3.5 & 8.0 \\
\hline Carbonate $\left(\mathrm{CO}_{3}\right) \ldots \ldots \ldots \ldots \ldots$ & & & & $\mathbf{0}$ \\
\hline Bicarbonate $\left(\mathrm{HCO}_{3}\right) \ldots . . . . . . . .$. & & & 64 & 79 \\
\hline Sulfate $\left(\mathrm{SO}_{4}\right) \ldots \ldots \ldots \ldots \ldots \ldots \ldots$ & & & 38 & 39 \\
\hline Chloride (Cl) ..................... & 3.8 & 5.1 & 5.1 & 7.0 \\
\hline Fluoride $(F)$ & & & & $\mathrm{d}_{1.0}$ \\
\hline Nitrate $\left(\mathrm{NO}_{3}\right)$ & 1.0 & 1.0 & 1.0 & 2.6 \\
\hline Dissolved solids $. . . . \ldots \ldots \ldots \ldots . .$. & 139 & 154 & 149 & 156 \\
\hline Hardness as $\mathrm{CaCO}_{3}$ : & & & & \\
\hline Total & 84 & 99 & 93 & 102 \\
\hline Noncarbonate ................... & 26 & 41 & 40 & 38 \\
\hline Color................ & - & -- & -- & \\
\hline pH $\ldots \ldots \ldots \ldots \ldots \ldots \ldots \ldots \ldots \ldots \ldots \ldots$ & 7.7 & 7.7 & 7.5 & 7.5 \\
\hline Specific conductance & & & & \\
\hline (micromhos at 25 C. $)$ & -- & -- & -- & 236 \\
\hline Turbidity ........................... & -- & -- & -- & -- \\
\hline Temperature (F.) .............. & 55 & 57 & 57 & -- \\
\hline Date of collection ................ & 1950 & 1950 & 1950 & $\begin{array}{c}\text { June } 30, \\
1952\end{array}$ \\
\hline
\end{tabular}

Regular determinations at treatment plants, 1950

\begin{tabular}{l|c|c|c|c|c|c|c|c|c|c|c|c}
\hline & \multicolumn{3}{|c|}{$\begin{array}{c}\text { Alkalinity } \\
\text { as CaCO } \\
\text { (ppm) }\end{array}$} & \multicolumn{3}{c|}{ pH } & \multicolumn{3}{c|}{$\begin{array}{c}\text { Hardness } \\
\text { as CaCO, } \\
\text { (ppm) }\end{array}$} & \multicolumn{2}{c}{$\begin{array}{c}\text { Turbidity } \\
\text { July 1948 to } \\
\text { July 1949 }\end{array}$} \\
\cline { 2 - 14 } & Av & Max & Min & Av & Max & Min & Av & Max & Min & Av & Max & Min \\
\hline Raw water..... & 59 & 82 & 41 & 7.7 & 7.9 & 7.5 & 84 & 114 & 70 & 69 & 165 & 21 \\
\hline $\begin{array}{l}\text { Dalecarlia Plant } \\
\text { Finished water. }\end{array}$ & 58 & 79 & 40 & 7.7 & 7.8 & 7.6 & 99 & 127 & 79 & .05 & .30 & .00 \\
\hline $\begin{array}{l}\text { McMillan Plant } \\
\text { Finished water.. }\end{array}$ & 62 & 74 & 37 & 7.5 & 7.8 & 7.3 & 93 & 120 & 77 & 0 & 0 & 0 \\
\hline
\end{tabular}

a Average of monthly analyses of composites of daily samples, Dalecarlia Reservoir outlet.

b Average of monthly analyses of composites of daily samples.

c Analysis by U. S. Geological Survey.

d Fluoridation of supply was begun June 23, 1952. 


\section{BRADENTON}

(Population, 13, 604)

Ownerśhip: Municipal.

Source: Lake Ward (Braden River).

Treatment: Aeration, coagulation with alum, sedimentation, rapid sand filtration, chlorination, and adjustment of $\mathrm{pH}$ with lime.

Rated capacity of treatment plant: 4,000,000 gpd.

Raw-water storage: Lake Ward.

Finished-water storage: 2,000,000 gal.

\section{ANALYSIS}

(Analysis, in parts per million, by U. S. Geological Survey)

\begin{tabular}{|c|c|c|c|}
\hline & $\begin{array}{l}\text { Finished } \\
\text { water }\end{array}$ & & $\begin{array}{c}\text { Finished } \\
\text { water }\end{array}$ \\
\hline $\begin{array}{l}\text { Silica }\left(\mathrm{SiO}_{2}\right) \ldots \ldots \ldots \ldots \ldots \\
\text { Iron }(\mathrm{Fe}) \quad \ldots \ldots \ldots \ldots \ldots \ldots \\
\text { Manganese }(\mathrm{Mn}) \ldots \ldots \ldots \ldots\end{array}$ & $\begin{array}{c}7.8 \\
.03 \\
.00\end{array}$ & $\begin{array}{l}\text { Hardness as } \mathrm{CaCO}_{3}: \\
\text { Total ...................... } \\
\text { Noncarbonate .......... }\end{array}$ & $\begin{array}{r}113 \\
79\end{array}$ \\
\hline $\begin{array}{l}\text { Calcium }(\mathrm{Ca}) \ldots \ldots \ldots \ldots \\
\text { Magnesium }(\mathrm{Mg}) \\
\text { Sodium }(\mathrm{Na}) \quad \ldots \ldots \ldots \ldots \ldots \\
\text { Potassium }(\mathrm{K}) \\
\text { Carbonate }\left(\mathrm{CO}_{3}\right) \\
\text { Bicarbonate }\left(\mathrm{HCO}_{3}\right)\end{array}$ & $\begin{array}{c}29 \\
10 \\
61 \\
2.5 \\
0 \\
42 \\
48 \\
116 \\
.2 \\
4.0 \\
308\end{array}$ & 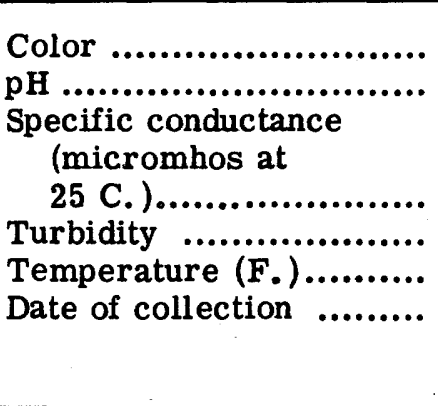 & $\begin{array}{r}555 \\
.4 \\
\operatorname{Jan} .19, \\
1952\end{array}$ \\
\hline
\end{tabular}

Regular determinations at treatment plant, 1951

\begin{tabular}{l|r|r|r|r|r|r|r|r|r|r|r|r}
\hline & \multicolumn{3}{|c|}{$\begin{array}{c}\text { Alkalinity } \\
\text { as } \begin{array}{c}\text { CaCO } \\
\text { (ppm) }\end{array}\end{array}$} & \multicolumn{4}{c|}{ pH } & \multicolumn{3}{c|}{$\begin{array}{c}\text { Hardness } \\
\text { as } \mathrm{CaCO}_{3} \\
\text { (ppm) }\end{array}$} & \multicolumn{3}{c}{ Turbidity } \\
\cline { 2 - 13 } & Av & Max & Min & Av & Max & Min & Av & Max & Min & Av & Max & Min \\
\hline Raw water......... & 42 & 58 & 30 & 7.0 & 7.2 & 6.8 & 67 & 91 & 43 & -- & -- & -- \\
Finished water... & 25 & 31 & 17 & 8.8 & 9.0 & 8.6 & 91 & 111 & 74 & -- & -- & -- \\
\hline
\end{tabular}




\section{CLEARWATER}

(Population, 15, 581)

Ownership: Municipal.

Source: 11 wells $(7,8,11$ to $13,16,18,19$, and 21 to 23$)$ ranging in depth from 135 to $296 \mathrm{ft}$ (depth not reported for wells 7 and 8).

Treatment: Partial aeration.

Storage: --

Analyses of samples from all of the wells indicate that the chloride content of the water from the wells ranged from 16 to $153 \mathrm{ppm}$.

\section{ANALYSES}

Analyses, in parts per million, by Black Laboratory, Inc., Gainesville, Fla.

\begin{tabular}{|c|c|c|c|c|c|}
\hline & Well 7 & Well 11 & Well 16 & Well $19^{a}$ & Well 21 \\
\hline 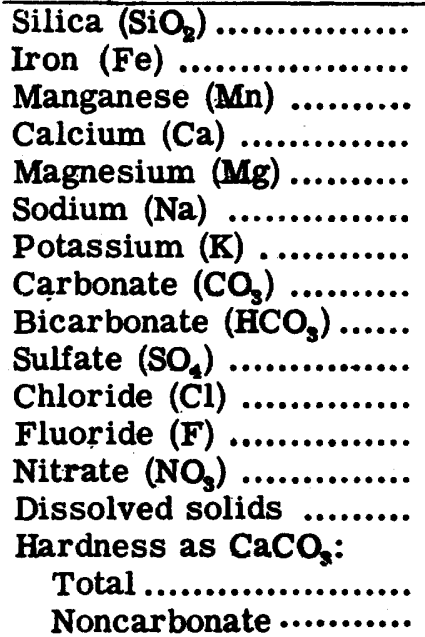 & 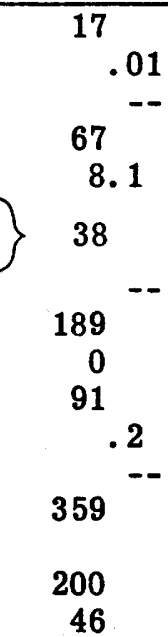 & $\begin{array}{r}21 \\
.14 \\
55 \\
6.1 \\
4.7 \\
183 \\
0 \\
16 \\
\quad .- \\
219 \\
163 \\
13\end{array}$ & $\begin{array}{r}14 \\
.03 \\
61 \\
7.3 \\
7.1 \\
194^{--} \\
0 \\
27 \\
.2 \\
261^{--} \\
182 \\
23\end{array}$ & $\begin{array}{c}24 \\
.05 \\
.00 \\
61 \\
9.7 \\
34 \\
.9 \\
0 \\
182 \\
3.0 \\
80 \\
.3 \\
.6 \\
336\end{array}$ & 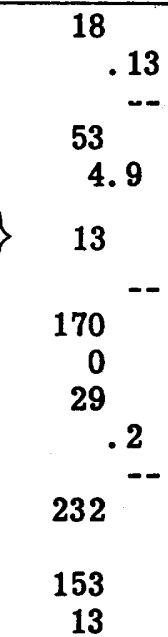 \\
\hline 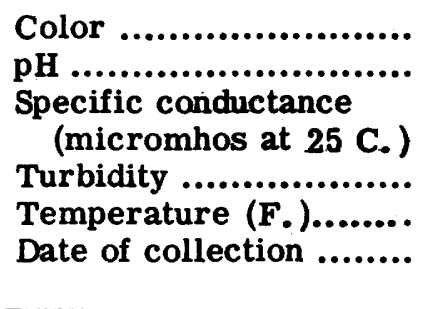 & $\begin{array}{r}7.6 \\
-- \\
.6 \\
\text { Mar. } 14, \\
1950\end{array}$ & 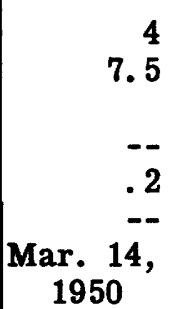 & $\begin{array}{r}7.5 \\
-- \\
.4 \\
-- \\
\text { Mar. } 14 \\
1950\end{array}$ & $\begin{array}{r}5 \\
7.5 \\
550 \\
8.2 \\
-- \\
\text { Nov. } 22, \\
1951\end{array}$ & 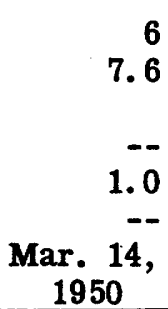 \\
\hline $\begin{array}{l}\text { Depth (feet) } \\
\text { Diameter (inches)........... } \\
\text { Date drilled ................ } \\
\text { Percent of supply ......... }\end{array}$ & $\begin{array}{r}-- \\
1924 \\
--\end{array}$ & $\begin{array}{r}206 \\
-- \\
-- \\
--\end{array}$ & $\begin{array}{r}135 \\
-- \\
1938 \\
--\end{array}$ & $\begin{array}{r}215 \\
10 \\
1941 \\
--\end{array}$ & $\begin{array}{r}296 \\
-- \\
1943 \\
--\end{array}$ \\
\hline
\end{tabular}

a Analyzed by U. S. Geological Survey.

CORAL GABLES

(Population, 19, 837)

Ownership: Supplied by Miami. (See Miami.) Population supplied, outside the city limits, about 21,200. Total population supplied, about 41,000. 
DAYTONA BEACH

(Population, 30, 187)

Ownership: Municipal; also supplies about 6,000 people outside the city limits, and a transient population of about 25,000. Total population supplied, about 61,000 .

Source: 26 wells ranging in depth from 165 to $220 \mathrm{ft}$.

Treatment: Softening with lime and soda ash, coagulation with alum, sedimentation, recarbonation, rapid sand filtration, and chlorination.

Rated capacity of treatment plants: Two plants: one, 4,000,000 gpd; the other, $1,000,000$ gpd.

Raw-water storage: None.

Finished-water storage: 2,850,000 gal.

\section{ANALYSES}

(Analyses, in parts per million, by Black Laboratory, Inc., Gainesville, Fla.)

\begin{tabular}{|c|c|c|c|}
\hline & $\begin{array}{l}\text { Raw water } \\
\text { (composite) }\end{array}$ & $\begin{array}{c}\text { Finished } \\
\text { water } \\
\text { (composite) }\end{array}$ & $\begin{array}{c}\text { Finished } \\
\text { water a } \\
\text { (composite) }\end{array}$ \\
\hline Silica $\left(\mathrm{SiO}_{2}\right) \quad \ldots \ldots \ldots \ldots \ldots \ldots \ldots \ldots$ & 17 & 22 & -- \\
\hline 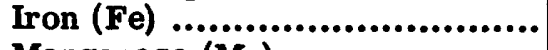 & .10 & .01 & .05 \\
\hline Manganese (Mn) .... & & -- & -- \\
\hline Calcium (Ca) ......................... & 105 & 11 & -- \\
\hline Magnesium (Mg)..................... & 20 & 14 & -- \\
\hline $\begin{array}{l}\text { Sodium }(\mathrm{Na}) \ldots \ldots \ldots \ldots \ldots \ldots \ldots \ldots \ldots \ldots \ldots \ldots \\
\text { Potassium (K) }\end{array}$ & 59 & 75 & 69 \\
\hline Carbonate $\left(\mathrm{CO}_{3}\right) \ldots \ldots \ldots \ldots \ldots \ldots$ & & 9 & 7 \\
\hline Bicarbonate $\left(\mathrm{HCO}_{3}\right) \ldots \ldots \ldots \ldots \ldots$ & 338 & 46 & 57 \\
\hline 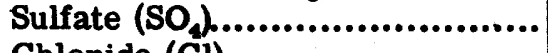 & & 13 & 15 \\
\hline Chloride $(\mathrm{Cl}) \ldots \ldots \ldots \ldots \ldots \ldots \ldots$ & & 140 & 112 \\
\hline Fluoride (F) $\ldots \ldots \ldots \ldots \ldots \ldots \ldots \ldots \ldots$ & .1 & .1 & .2 \\
\hline Nitrate $\left(\mathrm{NO}_{3}\right) \ldots \ldots \ldots \ldots \ldots \ldots \ldots \ldots$ & -- & & 4 \\
\hline Dissolved solids ................... & 618 & 314 & -- \\
\hline $\begin{array}{l}\text { Hardness as } \mathrm{CaCO}_{3} \text { : } \\
\text { Total }\end{array}$ & 345 & & \\
\hline 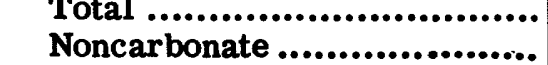 & $\begin{array}{r}345 \\
68\end{array}$ & $\begin{array}{l}85 \\
47\end{array}$ & $\begin{array}{l}82 \\
24\end{array}$ \\
\hline Color & & & \\
\hline pH.................. & 7.6 & 8.9 & 8.7 \\
\hline Specific cond & & & \\
\hline & - & & 491 \\
\hline 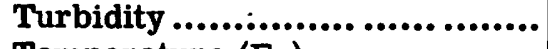 & & & -- \\
\hline Temperature (F.) $\ldots . . . . . . . . . . . .$. & & & -- \\
\hline Date of collection ..................... & Mar. 15, 1950 & Mar. 15, 1950 & Jan. 1952 \\
\hline
\end{tabular}

a Analysis by U. S. Geological Survey. 


\section{FORT LAUDERDALE}

(Population, 36, 328)

Ownership: Municipal; supplies about 4,500 people outside the city limits, and also about 50,000 seasonal visitors. Total maximum population supplied, about 91,000 .

Source: 18 wells ranging in depth from 130 to $185 \mathrm{ft}$.

Treatment: Aeration, softening with lime, sedimentation, rapid sand filtration, and chlorination.

Rated capacity of treatment plant: $14,000,000$ gpd.

Raw-water storage: None.

Finished-water storage: 3, 750,000 gal.

\section{ANALYSES}

(Analyses, in parts per million, by Black Laboratory, Inc., Gainesville; Fla.)

\begin{tabular}{|c|c|c|c|}
\hline & $\begin{array}{l}\text { Raw water }{ }^{a} \\
\text { (composite) }\end{array}$ & $\begin{array}{l}\text { Finished } \\
\text { water a } \\
\text { (composite) }\end{array}$ & $\begin{array}{l}\text { Finished } \\
\text { water b } \\
\text { (composite) }\end{array}$ \\
\hline 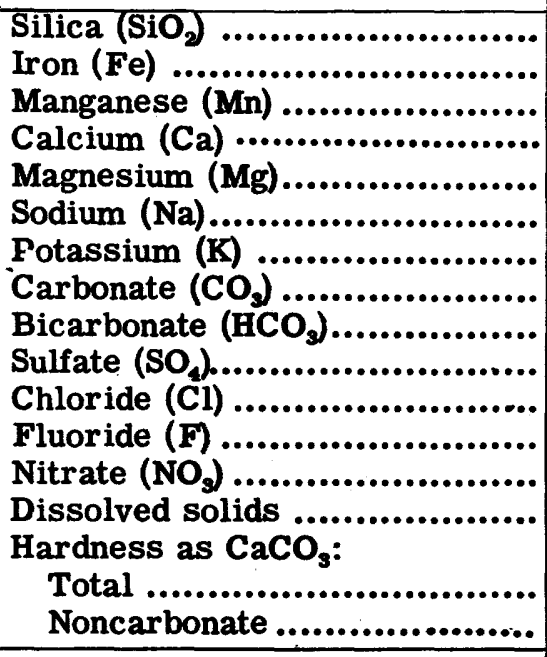 & $\begin{array}{r}22 \\
1.6 \\
92 \\
2.3 \\
8.6 \\
287^{--} \\
16^{-1} \\
314 \\
239 \\
4\end{array}$ & $\begin{array}{c}9.1 \\
.13 \\
23 \\
1.6 \\
3.0 \\
5 \\
39 \\
21 \\
.1 \\
130 \\
\\
\\
64 \\
23 \\
\end{array}$ & $\begin{array}{r}. \\
12 \\
12 \\
7 \\
7 \\
36 \\
10 \\
23 \\
.- \\
.2 \\
.2 \\
--\end{array}$ \\
\hline 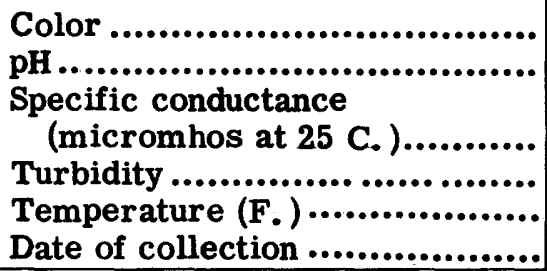 & $\begin{array}{r}59 \\
7.7 \\
-- \\
3 \\
-- \\
\text { Feb. } 8,1950\end{array}$ & $\begin{array}{r}22 \\
9.4 \\
-- \\
\text { Feb. } 8,1950\end{array}$ & $\begin{array}{r}29 \\
8.9 \\
169 \\
-- \\
-- \\
\text { Nov. } 23,1951 \\
\end{array}$ \\
\hline
\end{tabular}

Regular determinations at treatment plant, 1951

\begin{tabular}{l|r|r|r|r|r|r|r|r|r|r|r|r}
\hline & \multicolumn{3}{|c|}{$\begin{array}{c}\text { Alkalinity } \\
\text { as CaCO } \\
\text { (ppm) }\end{array}$} & \multicolumn{3}{c|}{ pH } & \multicolumn{3}{c|}{$\begin{array}{c}\text { Hardness } \\
\text { as CaCO } \\
\text { (ppm) }\end{array}$} & \multicolumn{3}{c}{ Turbidity } \\
\cline { 2 - 12 } & Av & Max & Min & Av & Max & Min & Av & Max & Min & Av & Max & Min \\
\hline Raw water........ & 250 & -- & - & 7.4 & -- & --260 & -- & -- & 0 & 0 & 0 \\
Finished water... & 40 & -- & -- & 9.6 & -- & -- & 53 & -- & -- & -- & --1 \\
\hline
\end{tabular}

${ }^{a}$ Wells 1 through 12 .

b 18 wells. Analysis by U. S. Geological Survey. 
GAINESVILLE

(Population, 26, 861)

Ownership: Municipal; also supplies the University of Florida.

Source: 4 wells (1 to 4) $365,407,421$, and $464 \mathrm{ft}$ deep.

Treatment: Aeration, prechlorination, coagulation with alum, softening with lime and soda ash, sedimentation, recarbonation, fluoridation, and rapid sand filtration.

Rated capacity of treatment plant: $3,500,000$ gpd.

Raw-water storage: None.

Finished-water storage: 1,500,000 gal.

The fluoride content of the raw water is about $0.3 \mathrm{ppm}$. The fluoride content of the treated water is maintained at about $0.7 \mathrm{ppm}$ from May 1 through October, and about $0.9 \mathrm{ppm}$ from November 1 to May.

\section{ANALYSES}

(Analyses, in parts per million, by Black Laboratory, Inc., Gainesville, Fla.)

\begin{tabular}{|c|c|c|c|}
\hline & Well 1 & Well 4 & $\begin{array}{l}\text { Finished } \\
\text { water } \\
\text { (composite) }\end{array}$ \\
\hline Silica $\left(\mathrm{SiO}_{2}\right) \ldots \ldots \ldots \ldots$ & 20 & 21 & 17 \\
\hline Iron $(\mathrm{Fe})$............................ & .10 & .03 & .0 \\
\hline Manganese (Mn) ..................... & -- & -- & -- \\
\hline Calcium (Ca) $\ldots \ldots \ldots \ldots \ldots \ldots . . . . . . . .$. & 51 & 55 & 17 \\
\hline Magnesium (Mg) ..................... & 13 & 13 & 3.6 \\
\hline Sodium (Na) ............................ & 11 & 3.8 & 22 \\
\hline Carbonate $\left(\mathrm{CO}_{3}\right) \ldots . . .$. & -- & -- & -- \\
\hline Bicarbonate $\left(\mathrm{HCO}_{3}\right) . . . . . . . . . . . . .$. & 200 & 210 & 58 \\
\hline 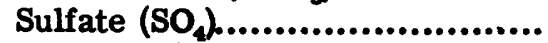 & 30 & 13 & 43 \\
\hline Chloride (Cl) $\ldots . \ldots \ldots \ldots \ldots \ldots \ldots \ldots \ldots$ & 7 & 9 & 9 \\
\hline Fluoride $(F)$. $\ldots \ldots \ldots \ldots \ldots \ldots \ldots \ldots \ldots$ & -- & .2 & .3 \\
\hline Nitrate $\left(\mathrm{NO}_{3}\right)$.......................... & & -- & -- \\
\hline Dissolved solids .................... & 246 & 248 & 140 \\
\hline Hardness as $\mathrm{CaCO}_{3}$ : & & & \\
\hline Total & 181 & 191 & 57 \\
\hline Noncarbonate ...................... & 17 & 19 & 10 \\
\hline Color ...................................... & 25 & & 0 \\
\hline pH & 7.6 & 7.8 & 8.5 \\
\hline $\begin{array}{l}\text { Specific conductance } \\
\text { (micromhos at } 25 \text { C.)..... }\end{array}$ & _ & & - \\
\hline Turbidity ............................. & - & .2 & .2 \\
\hline Temperature (F.) & & & -- \\
\hline Date of collection.................. & Mar. 13, 1948 & July 26, 1949 & Jan. 19,1948 \\
\hline
\end{tabular}

Regular determinations at treatment plant, 1951

\begin{tabular}{l|r|r|r|r|r|r|r|r|r|r|r|r}
\hline & \multicolumn{3}{|c|}{$\begin{array}{c}\text { Alkalinity } \\
\text { as } \begin{array}{c}\text { CaCO } \\
\text { (ppm) }\end{array}\end{array}$} & \multicolumn{3}{c|}{ pH } & \multicolumn{3}{c|}{$\begin{array}{c}\text { Hardness } \\
\text { as CaCO } \\
\text { (ppm) }\end{array}$} & \multicolumn{3}{c}{ Turbidity } \\
\cline { 2 - 12 } & Av & Max & Min & Av & Max & Min & Av & Max & Min & Av & Max & Min \\
\hline Raw water........ & 180 & -- & - & 7.8 & -- & -- & 200 & -- & -- & -- & -- & -- \\
Finished water... & 55 & -- & -- & 8.4 & -- & -- & 65 & -- & -- & -- & -- & - \\
\hline
\end{tabular}




\section{HIALEAH}

(Population, 19, 676)

Ownership: Supplied by Miami. (See Miami.)

\section{HOLLYWOOD}

(Population, 14, 351)

Ownership: Municipal. Population supplied, highly variable, in winter months, about 35,000; in summer, about 21,000.

Source: 5 wells (1 to 5). Wells 1, 2, and 4 are each $85 \mathrm{ft}$ deep. (Depths not reported for wells 3 and 5).

Treatment: Zeolite softening, chlorination, stabilization, and chlorination.

Rated capacity of treatment plant: 4,200,000 gpd.

Raw-water storage: 515,000 gal.

Finished-water storage: 2,000,000 gal.

\section{ANALYSES}

(Analyses, in parts per million, by U. S. Geological Survey)

\begin{tabular}{|c|c|c|c|c|c|}
\hline & $\underset{\text { water }}{\text { Raw }}$ & $\begin{array}{c}\text { Finished } \\
\text { water a }\end{array}$ & & $\begin{array}{c}\text { Raw } \\
\text { water a }\end{array}$ & $\begin{array}{l}\text { Finished } \\
\text { water a }^{\text {a }}\end{array}$ \\
\hline $\begin{array}{l}\text { Silica }\left(\mathrm{SiO}_{2}\right) \ldots \ldots \ldots \\
\text { Iron }(\mathrm{Fe}) \ldots \ldots . . . . . . . . \\
\text { Manganese }(\mathrm{Mn}) \ldots . .\end{array}$ & .08 & $\begin{array}{r}-- \\
.06 \\
--\end{array}$ & $\begin{array}{r}\text { Hardness as } \mathrm{CaCO}_{3} \text { : } \\
\text { Total ................... } \\
\text { Noncarbonate..... }\end{array}$ & $\begin{array}{r}273 \\
22\end{array}$ & $\begin{array}{r}86 \\
0\end{array}$ \\
\hline $\begin{array}{l}\text { Calcium (Ca) .......... } \\
\text { Magnesium (Mg)..... }\end{array}$ & $\begin{array}{r}102 \\
4.4\end{array}$ & $\begin{array}{l}30 \\
2.7\end{array}$ & Color ..................... & 25 & 19 \\
\hline $\begin{array}{l}\text { Sodium (Na) ........... } \\
\text { Potassium (K) ....... }\end{array}$ & 31 & 116 & pH ........................ & 6.8 & 6.7 \\
\hline $\begin{array}{l}\text { Carbonate }\left(\mathrm{CO}_{3}\right) \ldots . . . \\
\text { Bicarbonate }\left(\mathrm{HCO}_{3}\right)\end{array}$ & $\begin{array}{r}0 \\
306\end{array}$ & $\begin{array}{r}0 \\
300\end{array}$ & 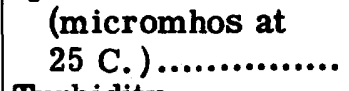 & 632 & 620 \\
\hline Sulfate $\left(\mathrm{SO}_{4}\right) \ldots \ldots \ldots$ & $\begin{array}{l}28 \\
42\end{array}$ & 35 & Turbidity ................ & -- & -- \\
\hline $\begin{array}{l}\text { Chloride }(\mathrm{Cl}) \ldots \ldots \ldots \\
\text { Fluoride }(\mathrm{F}) \ldots \ldots \ldots \\
\text { Nitrate }\left(\mathrm{NO}_{3}\right) \ldots \ldots . . . . \\
\text { Dissolved solids...... }\end{array}$ & $\begin{array}{r}.1 \\
359^{.7}\end{array}$ & $\begin{array}{r}40 \\
372^{.3}\end{array}$ & $\begin{array}{l}\text { Temperature (F.)... } \\
\text { Date of collection ... }\end{array}$ & $\begin{array}{c}\text { Mar. 29, } \\
1948\end{array}$ & $\begin{array}{c}\text { Mar.29, } \\
1948\end{array}$ \\
\hline
\end{tabular}

Regular determinations at treatment plant, 1950

\begin{tabular}{|c|c|c|c|c|c|c|c|c|c|c|c|c|}
\hline & \multicolumn{3}{|c|}{$\begin{array}{c}\text { Alkalinity } \\
\text { as CaCO } \\
\text { (ppm) }\end{array}$} & \multicolumn{3}{|c|}{ pH } & \multicolumn{3}{|c|}{$\begin{array}{l}\text { Hardness } \\
\text { as } \mathrm{CaCO} \\
(\mathrm{ppm})\end{array}$} & \multicolumn{3}{|c|}{$\begin{array}{c}\text { Tempera- } \\
\text { ture } \\
\left({ }^{\circ} \mathrm{F} .\right)\end{array}$} \\
\hline & Av & $\operatorname{Max}$ & Min & Av & Max & Min & Av & Max & $\mathrm{M}$ in & Av & $\operatorname{Max}$ & $\operatorname{Min}$ \\
\hline $\begin{array}{l}\text { Raw water......... } \\
\text { Finished water... }\end{array}$ & $\begin{array}{r}225 \\
--\end{array}$ & $\overline{140}$ & $\overline{87}$ & $\begin{array}{l}7.2 \\
7.2\end{array}$ & $\begin{array}{l}-- \\
--\end{array}$ & $\begin{array}{l}-- \\
--\end{array}$ & $\begin{array}{r}266 \\
--\end{array}$ & -- & -- & $\begin{array}{l}56 \\
60\end{array}$ & -- & - \\
\hline
\end{tabular}

a Composite. 


\section{JACKSONVILLE}

(Population, 204, 517)

Ownership: Municipal; supplies also about 20,000 people outside the city limits.

Total population supplied, about 224,500 .

Source: 44 artesian wells most of which range in depth from about 1,000 to about $1,300 \mathrm{ft}$.

Treatment: Aeration at reservoirs, and chlorination.

Storage: Ground reservoirs, 18,000,000 gal; elevated tanks, 3,000,000 gal.

All of the wells yield water of similar chemical composition. Hydrogen sulfide $\left(\mathrm{H}_{2} \mathrm{~S}\right)$ in quantities of 1.5 to $2.5 \mathrm{ppm}$ is present in all the well supplies. The fluoride content is reported to be 0.6 to $0.7 \mathrm{ppm}$.

\section{ANALYSES}

(Analyses, in parts per million, by Department of Chemistry, University of Fla.)

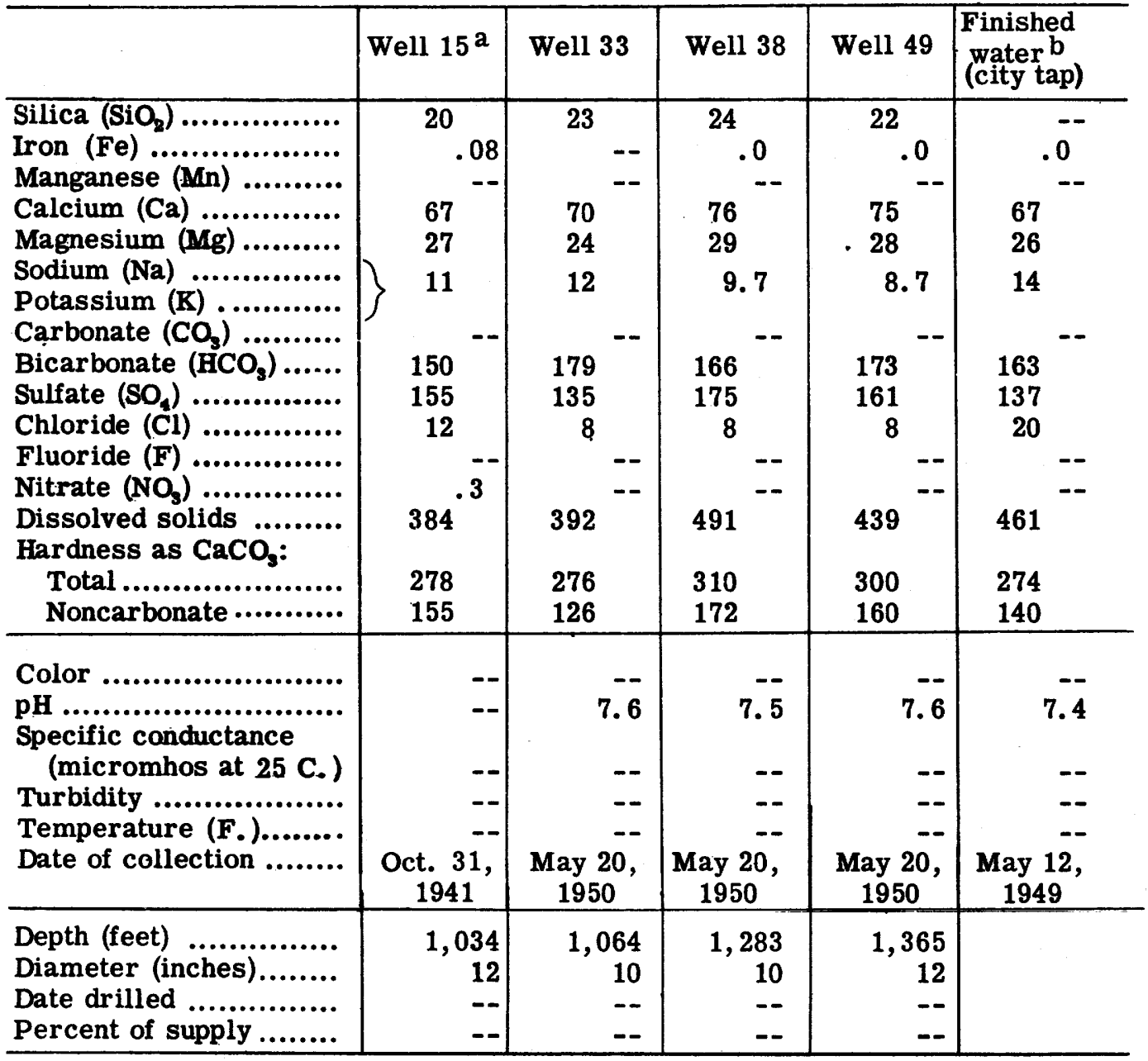

a Analysis by U. S. Geological Survey.

b Analysis by Fla. State Board of Health, Tallahassee, Fla. 


\section{KEY WEST}

(Population, 26, 433)

Ownership: U. S. Navy; also supplies about 5,000 people outside the city limits, and other communities on the Florida Keys. Total population supplied, about 35,000 .

Source: 4 wells (1 to 4$) 62,62,48$, and $53 \mathrm{ft}$ deep. The wells are located at Florida City and the water is piped to Key West, a distance of 127 miles. Treatment: Softening with lime, sedimentation, filtration, and chlorination. Rated capacity of treatment plant: 3,000,000 gpd.

Raw-water storage: None.

Finished-water storage: 300,000 gal.

\section{ANALYSIS}

(Analysis, in parts per million, by U. S. Geological Survey)

\begin{tabular}{|c|c|c|c|c|c|c|c|c|c|c|c|c|}
\hline & \multicolumn{2}{|c|}{$\begin{array}{c}\text { Wells, } \\
\text { Finished } \\
\text { water } \\
\text { (composite) }\end{array}$} & & \multicolumn{3}{|c|}{$\begin{array}{c}\text { Wells, } \\
\text { Finished } \\
\text { water } \\
\text { (composite) }\end{array}$} \\
\hline \multirow{9}{*}{\multicolumn{3}{|c|}{ 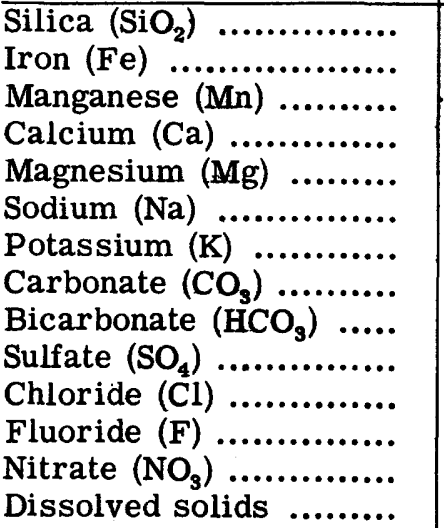 }} & \multirow{2}{*}{\multicolumn{2}{|c|}{$\begin{array}{r}3.3 \\
.12 \\
.00\end{array}$}} & \multicolumn{6}{|c|}{$\begin{array}{l}\text { Hardness as } \mathrm{CaCO}_{3}: \\
\text { Total ....................... } \\
\text { Noncarbonate ........... }\end{array}$} & \multicolumn{2}{|r|}{$\begin{array}{l}60 \\
10\end{array}$} \\
\hline & & & & & \multirow{8}{*}{\multicolumn{5}{|c|}{ 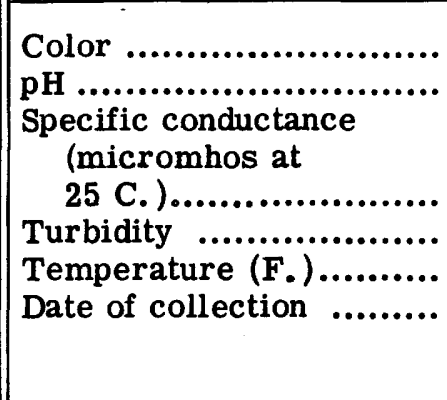 }} & \multirow{2}{*}{\multicolumn{3}{|c|}{$\begin{array}{r}2 \\
8.1\end{array}$}} \\
\hline & & & \multicolumn{2}{|c|}{$\begin{array}{l}1.8 \\
6.3\end{array}$} & & & & & & & & \\
\hline & & & \multicolumn{2}{|r|}{$0^{.5}$} & & & & & & \multirow{2}{*}{\multicolumn{3}{|c|}{150}} \\
\hline & & & & 1 & & & & & & & & \\
\hline & & & & 6.5 & & & & & & \multirow{4}{*}{\multicolumn{3}{|c|}{$\underset{1952}{\operatorname{Jan} 3 \overline{0}}$}} \\
\hline & & & & 10 & & & & & & & & \\
\hline & & & & $\begin{array}{r}1 \\
2\end{array}$ & & & & & & & & \\
\hline & & & & $5^{\circ}$ & & & & & & & & \\
\hline \multicolumn{13}{|c|}{ Regular determinations at treatment plant, 1951} \\
\hline & \multicolumn{3}{|c|}{$\begin{array}{l}\text { Alkalinity } \\
\text { as } \mathrm{CaCO}_{3} \\
\text { (ppm) }\end{array}$} & \multicolumn{3}{|c|}{ pH } & \multicolumn{3}{|c|}{$\begin{array}{l}\text { Hardness } \\
\text { as } \mathrm{CaCO}_{3} \\
\text { (ppm) }\end{array}$} & \multicolumn{3}{|c|}{ Turbidity } \\
\hline & & & & & & & & & & & & \\
\hline & Av & $\operatorname{Max}$ & Min & Av & $\operatorname{Max}$ & Min & Av & $\operatorname{Max}$ & Min & Av & Max & Min \\
\hline Raw & 180 & 185 & 170 & 7.4 & 7.4 & 7.4 & 180 & 185 & 170 & 0 & 0 & 0 \\
\hline & 86 & 115 & 65 & 8.4 & & & 76 & 100 & 54 & 0 & 0 & 0 \\
\hline
\end{tabular}




\section{LAKELAND \\ (Population, 30, 851)}

Ownership: Municipal; supplies also about 5,000 people outside the city limits.

Total population supplied, about 35,900.

Source: 7 deep wells (1 to 7). The depths of wells 1 to 5 are reported to be 741 , $846,1,201,683$, and $828 \mathrm{ft}$. The yield of well 5 is reported to be $4,500 \mathrm{gpm}$. Wells 1 and 2 are used in emergencies.

Treatment: Aeration and chlorination.

Rated capacity of treatment plant: --

Raw-water storage: 800,000 gal.

Finished-water storage: 750,000 gal.

\section{ANALYSES}

(Analyses, in parts per million, by U. S. Geological Survey)

\begin{tabular}{|c|c|c|c|}
\hline & Well 3 a & Well $4^{\text {b }}$ & Well 5 \\
\hline 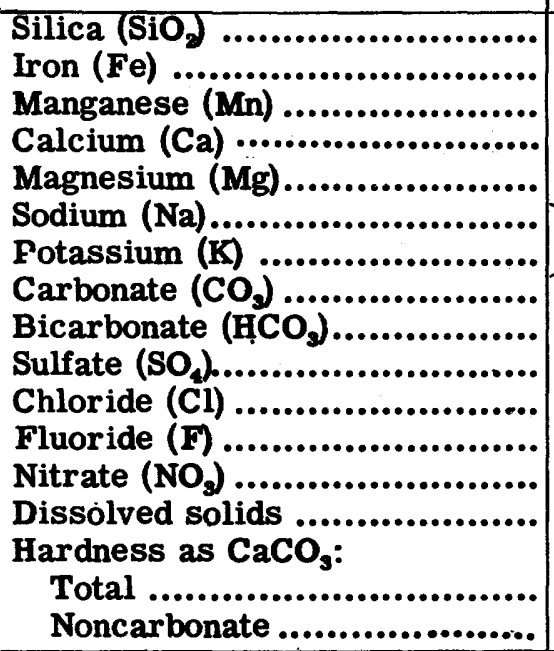 & \begin{tabular}{rrr}
22 & \\
& .16 \\
& -- \\
91 & \\
21 & \\
7.4 \\
7. & \\
\multicolumn{2}{c}{--} \\
250 & - \\
100 & \\
15 & \\
& -- \\
& -- \\
425 & \\
& \\
314 & \\
109 &
\end{tabular} & \begin{tabular}{rr}
\multicolumn{2}{c}{.05} \\
54 & -- \\
18 & \\
8.8 \\
266 & \\
0 & -- \\
7 & \\
& -- \\
245 & -- \\
209 & \\
0 &
\end{tabular} & $\begin{array}{c}19 \\
.02 \\
54 \\
54 \\
16 \\
6.8 \\
1.0 \\
0 \\
242 \\
2.0 \\
10 \\
.4 \\
.2 \\
236 \\
201 \\
2\end{array}$ \\
\hline 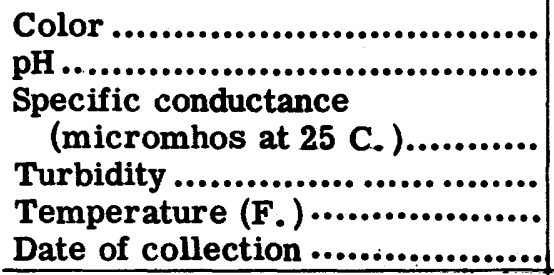 & Feb. 18,1935 & $\begin{array}{r}12 \\
7.2 \\
-- \\
-- \\
-\overline{-} \\
\text { Mar. } 18,1946\end{array}$ & $\begin{array}{r}10 \\
7.2 . \\
393 \\
7.0 \\
80 \\
\text { Nov. } 24,1951 \\
\end{array}$ \\
\hline 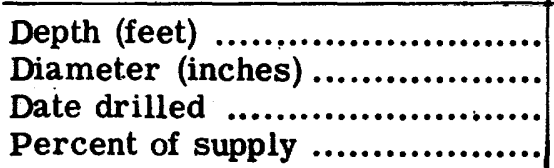 & $\begin{array}{r}1,201 \\
18 \\
1926 \\
--\end{array}$ & $\begin{array}{r}683 \\
-- \\
-- \\
--\end{array}$ & $\begin{array}{r}828 \\
24 \\
1945 \\
--\end{array}$ \\
\hline
\end{tabular}

a Analysis by Southern Analytical Laboratory.

b Analysis by Florida State Board of Health, Jacksonville, Fla. 


\section{MIAMI \\ (Population, 249, 276)}

Ownership: Municipal; also supplies Coral Gables, El Portal, Hialeah, Miami Beach, Miami Shores, Miami Springs, Surfside, and other communities. Total population supplied, about 350,000 .

Source: 22 wells ( 1 to 8,11 to 24 ) 62 to $110 \mathrm{ft}$ deep serving the Hialeah Plant, and 4 wells (1 to 4) 98 to $101 \mathrm{ft}$ deep serving the Southwest Plant.

Treatment: Hialeah Plant: Softening with lime, sedimentation, recarbonation, rapid sand filtration, chlorination, and fluoridation. Southwest Plant: . Softening with lime, sedimentation, and chlorination.

Rated capacity of treatment plants: Hialeah Plant, 60,000,000 gpd; Southwest Plant, 40, 000, 000 gpd.

Raw-water storage: None.

Finished-water storage: 24,000,000 gal.

The Southwest supply system is under development. Ultimately it is to provide $80,000,000$ gpd from the new well field located about 1 mile west of the County Hospital at Kindall and 4 miles west of the Southwest treatment plant at Galloway Road and Seaboard Airline Railroad, which is $6 \frac{1}{2}$ miles from the southwest section of Miami. The treatment for the water will be similar to that given at the Hialeah plant.

The water from the wells of both fields is similar in chemical composition.

\section{ANALYSES}

(Analyses, in parts per million, by City of Miami Water Department)

\begin{tabular}{|c|c|c|c|c|c|}
\hline & $\begin{array}{c}\text { Well 1, } \\
\text { Hialeah } \\
\text { Plant }\end{array}$ & $\begin{array}{c}\text { Well 24, } \\
\text { Hialeah } \\
\text { Plant }\end{array}$ & $\begin{array}{l}\text { Well 2, } \\
\text { South- } \\
\text { west } \\
\text { Field }\end{array}$ & $\begin{array}{c}\text { Raw } \\
\text { water } \\
\text { composite) }\end{array}$ & $\begin{array}{c}\text { Finished } \\
\text { water } \\
\text { (composite) }\end{array}$ \\
\hline Silica $\left(\mathrm{SiO}_{2}\right) \ldots \ldots \ldots \ldots \ldots$ & 6.8 & 10 & 4.8 & 9.0 & 9.0 \\
\hline Iron $(\mathrm{Fe}) \ldots \ldots \ldots \ldots \ldots \ldots$ & 1.7 & 1.2 & .62 & .9 & .02 \\
\hline Manganese $(\mathbf{M n})$........... & -- & & -- & -- & -- \\
\hline Calcium (Ca) ............... & & 89 & 95 & & 25 \\
\hline Magnesium (Mg) ........... & 4.9 & 4.4 & 2.9 & 7.1 & 4.0 \\
\hline Sodium (Na) ............... & 13 & 8.3 & 3.2 & 17 & 27 \\
\hline Carbonate $\left(\mathrm{CO}_{3}\right)$ & 0 & 0 & 0 & 0 & 3 \\
\hline Bicarbonate $\left(\mathrm{HCO}_{3}\right) \ldots . .$. & 255 & 278 & 264 & 268 & 49 \\
\hline Sulfate $\left(\mathrm{SO}_{4}\right) \ldots \ldots \ldots \ldots \ldots$ & 39 & 7.4 & 18 & 37 & 40 \\
\hline Chloride (CI) .............. & 22 & 16 & 15 & 35 & 35 \\
\hline Fluoride $(F)$............... & - & -- & -- & & a. 2 \\
\hline Nitrate $\left(\mathrm{NO}_{3}\right) \ldots \ldots \ldots \ldots \ldots$ & & -- & -- & 2.2 & 2.2 \\
\hline Dissolved solids ......... & 330 & 310 & 275 & 350 & 190 \\
\hline Hardness as $\mathrm{CaCO}_{3}$ : & & & & & \\
\hline Total.................. & 252 & 240 & 249 & 274 & 79 \\
\hline Noncarbonate ............ & 43 & 12 & 32 & 56 & 34 \\
\hline 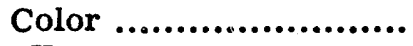 & - & -- & -- & 80 & 23 \\
\hline pH & 7.3 & 7.2 & 7.2 & 7.3 & 8.7 \\
\hline Specific conductance & & & & & \\
\hline (micromhos at $25 \mathrm{C}$. ) & -- & -- & - & - & -- \\
\hline Turbidity............$\ldots \ldots$. & & -- & - & - & -- \\
\hline Temperature (F.)........ & $10 \overline{4}$ & $10 \overline{1}$ & $10 \overline{\overline{1}}$ & hovoman & 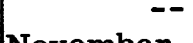 \\
\hline Date of analysis .... & 1946 & 1951 & 1951 & $\begin{array}{c}\text { November, } \\
1948\end{array}$ & $\begin{array}{c}\text { November, } \\
1948\end{array}$ \\
\hline
\end{tabular}

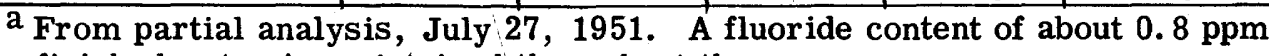
in the finished water is maintained throughout the year. 


\section{MIAMI--Continued}

Regular determinations at treatment plant, 1951

\begin{tabular}{|c|c|c|c|c|c|c|c|c|c|c|c|c|}
\hline & \multicolumn{3}{|c|}{$\begin{array}{l}\text { Alkalinity } \\
\text { as } \mathrm{CaCO}_{3} \\
\text { (ppm) }\end{array}$} & \multicolumn{3}{|c|}{ pH } & \multicolumn{3}{|c|}{$\begin{array}{l}\text { Hardness } \\
\text { as } \mathrm{CaCO}_{3} \\
\text { (ppm) }\end{array}$} & \multicolumn{3}{|c|}{ Turbidity } \\
\hline & Av & $\operatorname{Max}$ & Min & Av & $\operatorname{Max}$ & Min & Av & $\operatorname{Max}$ & Min & Av & $\operatorname{Max}$ & Min \\
\hline $\begin{array}{l}\text { Raw water......... } \\
\text { Finished water... }\end{array}$ & $\begin{array}{r}220 \\
43\end{array}$ & $\begin{array}{r}230 \\
52 \\
\end{array}$ & $\begin{array}{r}215 \\
36 \\
\end{array}$ & $\begin{array}{l}7.3 \\
8.8 \\
\end{array}$ & $9 .--$ & \begin{tabular}{|l|}
7.2 \\
8.4 \\
\end{tabular} & $\begin{array}{r}260 \\
85 \\
\end{array}$ & $\begin{array}{l}265 \\
100 \\
\end{array}$ & $\begin{array}{r}250 \\
80 \\
\end{array}$ & $\begin{array}{r}0.1 \\
.1 \\
\end{array}$ & $\begin{array}{l}-- \\
--\end{array}$ & -- \\
\hline
\end{tabular}

\section{MIAMI BEACH}

(Population, 46, 282)

Ownership: Supplied by Miami. (See Miami.) 


\section{ORLANDO}

(Population, 52, 367)

Ownership: Municipal; also supplies about 17,000 people outside the city limits.

Total population supplied, about 69,000 .

Source: Lake Underhill, Lake Ivanhoe, and 1 well $908 \mathrm{ft}$ deep. Well water is pumped into Lake Underhill to maintain the water level of the lake. Lake water is then pumped to the treatment plant.

Treatment: Aeration, coagulation with alum and activated silica, lime, sedimentation, rapid sand filtration, and chlorination.

Rated capacity of treatment plant: 20,000, 000 gpd.

Raw-water storage: 737, 000, 000 gal.

Finished-water storage: 5, 000,000 gal.

\section{ANALYSES}

(Analyses, in parts per million, by BlackLaboratory, Inc., Gainesville, Fla.).

\begin{tabular}{|c|c|c|c|c|}
\hline & $\begin{array}{c}\text { Lake } \\
\text { Underhill }\end{array}$ & $\begin{array}{c}\text { Lake } \\
\text { Ivanhoe }\end{array}$ & Well 1 & $\begin{array}{l}\text { Finished } \\
\text { water } \\
\text { (composite) }\end{array}$ \\
\hline 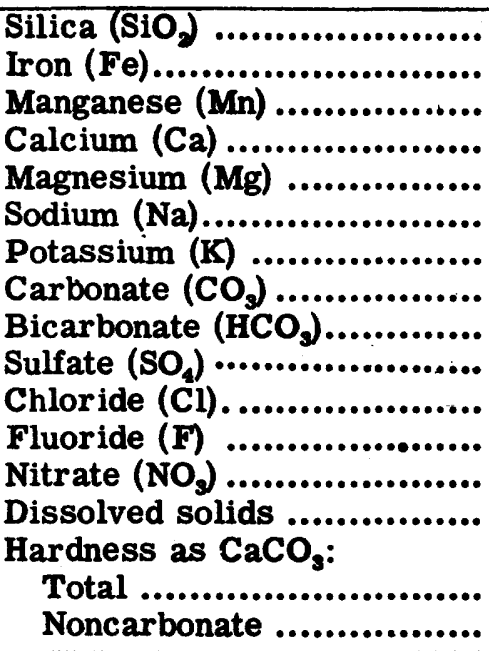 & $\begin{array}{c}1.4 \\
.05 \\
27^{--} \\
3.2 \\
12 \\
102^{--} \\
6.5 \\
12 \\
\quad-- \\
125^{--} \\
81 \\
0\end{array}$ & 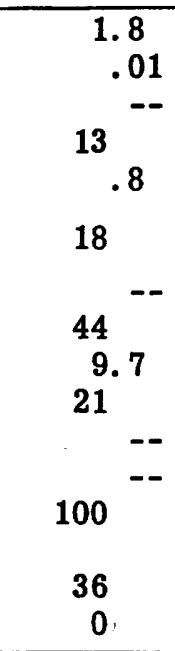 & $\begin{array}{c}5.7 \\
.01 \\
30 \\
7.4 \\
11 \\
132 \\
4.2 \\
12 \\
.1 \\
135^{--} \\
105 \\
0\end{array}$ & $\begin{array}{c}4.0 \\
.06 \\
39 \\
6.3 \\
20^{--} \\
93^{--} \\
60^{-1} \\
20 \\
180^{--} \\
123 \\
47\end{array}$ \\
\hline 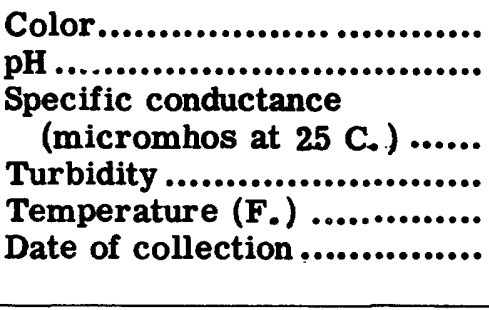 & $\begin{array}{r}7.4 \\
-- \\
-- \\
-- \\
\text { Dec. } 15, \\
1947\end{array}$ & $\begin{array}{r}7.3 \\
-- \\
-- \\
\text { Dec. } 15, \\
1947^{--}\end{array}$ & $\begin{array}{c}-- \\
\text { Apr. } 21, \\
1948\end{array}$ & $\begin{array}{l}-- \\
7.3 \\
-- \\
-- \\
--\end{array}$ \\
\hline
\end{tabular}

Regular determinations at treatment plant, 1951

\begin{tabular}{|c|c|c|c|c|c|c|c|c|c|c|c|c|}
\hline & \multicolumn{3}{|c|}{$\begin{array}{l}\text { Alkalinity } \\
\text { as } \mathrm{CaCO}_{3} \\
\text { (ppm) }\end{array}$} & \multicolumn{3}{|c|}{$\mathrm{pH}$} & \multicolumn{3}{|c|}{$\begin{array}{c}\text { Hardness } \\
\text { as } \mathrm{CaCO} \mathrm{O}_{3} \\
(\mathrm{ppm})\end{array}$} & \multicolumn{3}{|c|}{ Turbidity } \\
\hline & Av & $\operatorname{Max}$ & Min & Av & $\operatorname{Max}$ & Min & Av & Max & Min & Av & $\operatorname{Max}$ & Min \\
\hline $\begin{array}{l}\text { Raw water......... } \\
\text { Finished water... }\end{array}$ & $\begin{array}{l}95 \\
80 \\
\end{array}$ & $\begin{array}{l}99 \\
87\end{array}$ & $\begin{array}{l}74 \\
57 \\
\end{array}$ & $\begin{array}{l}8.4 \\
8.4\end{array}$ & $\begin{array}{l}8.8 \\
8.5 \\
\end{array}$ & $\begin{array}{l}7.6 \\
8.1 \\
\end{array}$ & $\begin{array}{r}98 \\
105 \\
\end{array}$ & $\begin{array}{l}105 \\
115 \\
\end{array}$ & $\begin{array}{l}86 \\
95\end{array}$ & $\begin{array}{r}2.0 \\
.5 \\
\end{array}$ & $\begin{array}{r}2.7 \\
.5 \\
\end{array}$ & $\begin{array}{r}1.4 \\
.5\end{array}$ \\
\hline
\end{tabular}


PANAMA CITY

(Population, 25, 814)

Ownership: Municipal; also supplies about 500 people outside the city limits.

T'otal population supplied, about 26,300 .

Source: 2 well fields: Millville, 4 wells ( 1 to 4 ) 80 to $130 \mathrm{ft}$ deep; St. Andrews, 7 wells, each $600 \mathrm{ft}$ deep.

Treatment: Millville plant: Softening with lime, sedimentation, recarbonation, filtration, and chlorination. St. Andrews: Chlorination only.

Rated capacity of treatment plant: Millville plant, 1, 000,000 gpd.

Raw-water storage: St. Andrews water, 1,000,000 gal.

Finished-water storage: Millville water: 3 elevated tanks, 100,000 gal each; clear wells, 400, 000 gal.

\section{ANALYSES}

(Analyses, in parts per million, by U. S. Geological Survey)

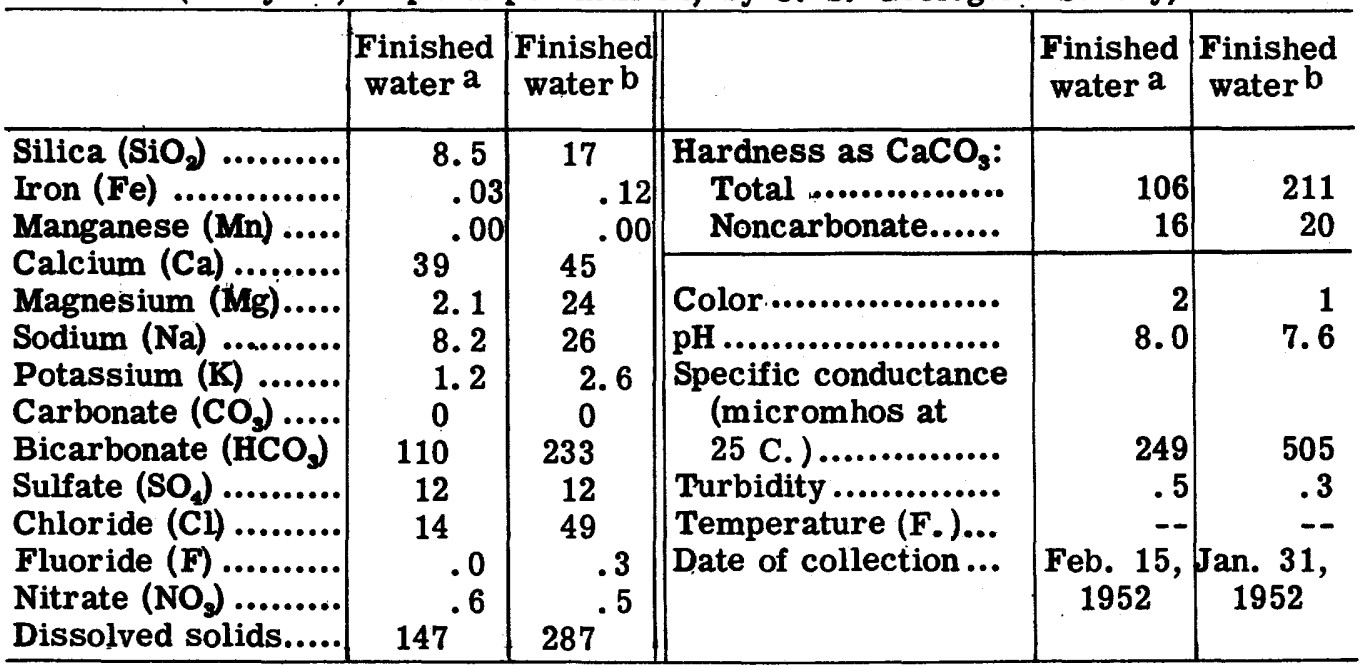

a Composite, Millville well field.

b Composite, St. Andrews well field. 


\section{PENSACOLA}

(Population, 43, 479)

Ownership: Municipal; total population supplied, about 48, 000 .

Source: 6 wells: Main Plant wells (6 to 9) $245,240,169$, and $252 \mathrm{ft}$ deep; West Plant well, $226 \mathrm{ft}$ deep; East Plant well, $260 \mathrm{ft}$ deep.

Treatment: Aeration, $\mathrm{pH}$ control, and chlorination.

Rated capacity of plants: $19,440,000$ gpd.

Raw-water storage: 1,600,000 gal.

Finished-water storage: 1,000,000 gal.

\section{ANALYSIS}

(Analysis, in parts per million, by U. S. Geological Survey)

\begin{tabular}{|c|c|c|c|}
\hline & $\begin{array}{l}\text { Finished } \\
\text { water a }\end{array}$ & & $\begin{array}{c}\text { Finished } \\
\text { water a }\end{array}$ \\
\hline \multirow{2}{*}{ 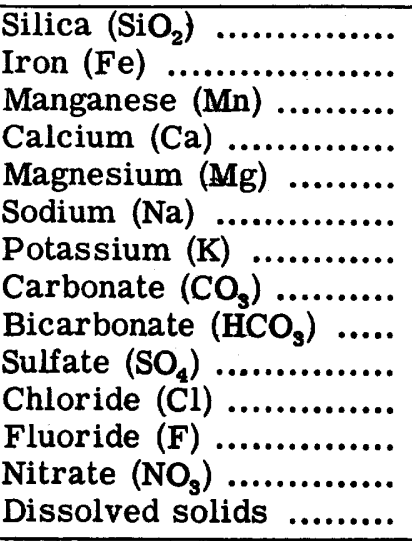 } & \multirow{2}{*}{$\begin{array}{c}9.0 \\
.0 \\
.00 \\
5.0 \\
1.8 \\
7.2 \\
.6 \\
0 \\
11 \\
5.8 \\
9.8 \\
.0 \\
8.5 \\
56\end{array}$} & $\begin{array}{l}\text { Hardness as } \mathrm{CaCO}_{3}: \\
\text { Total ...................... } \\
\text { Noncarbonate .......... }\end{array}$ & $\begin{array}{l}20 \\
11\end{array}$ \\
\hline & & 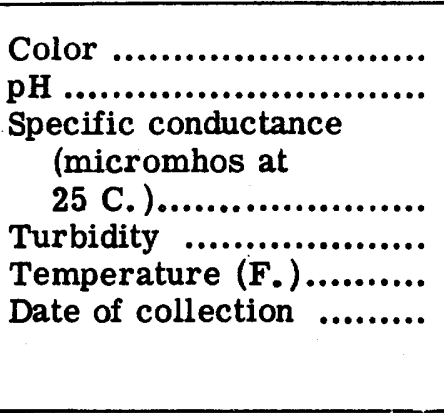 & $\begin{array}{r}87.0 \\
3.0 \\
-- \\
\text { Jan. } 5 \text {, } \\
1952\end{array}$ \\
\hline \multicolumn{3}{|c|}{ 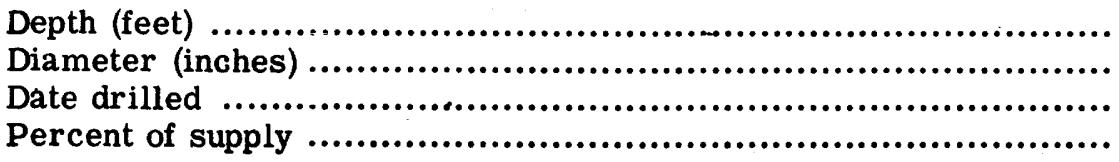 } & $\begin{array}{r}245,252 \\
30, \quad 16 \\
1940,1945\end{array}$ \\
\hline
\end{tabular}

a Composite, wells 6 and 9. 
ST. PETERSBURG

(Population, 96, 738)

Ownership: Municipal; supplies also Gulport, Pinellas Park, Bay Pines Hospital, and other consumers. Total population regularly supplied, about 110, 000 . Population supplied during winter tourist season may reach a peak total of $275,000$.

Source: 14 wells ranging in depth from 300 to $350 \mathrm{ft}$ located near Cosme in northwest part of Hillsborough County. Emergency supplies from Mirror Lake (Plant) and Crescent Lake (Plant).

Treatment: Softening with lime, coagulation with ferric sulfate, sedimentation, rapid sand filtration, and chlorination.

Rated capacity of treatment plant: $12,000,000$ gpd.

Raw-water storage: None.

Finished-water storage: 330,000 gal.

Analyses of samples from all of the wells indicate that the wells yield water of about the same composition.

\section{ANALYSES}

(Analyses, in parts per million, by Black Laboratory, Inc., Gainesville, Fla.)

\begin{tabular}{|c|c|c|c|c|c|}
\hline & Well 1C & Well 5 & Well 7A & $\underset{\text { water }}{\text { Raw }}$ & $\begin{array}{l}\text { Finished } \\
\text { water a }\end{array}$ \\
\hline 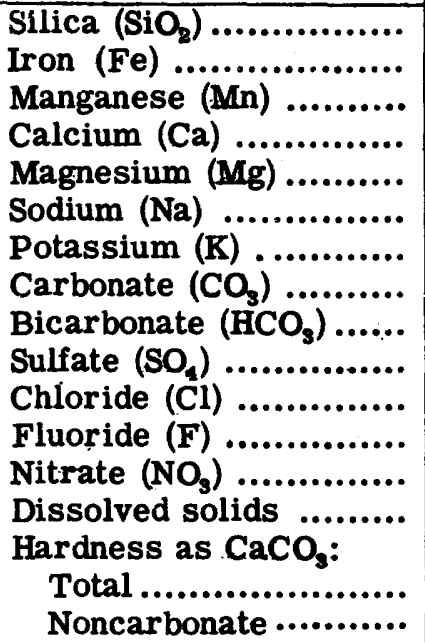 & 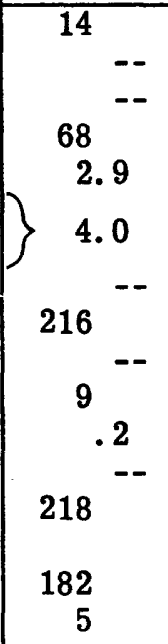 & \begin{tabular}{r}
16 \\
76 \\
4. \\
$4 .-$ \\
4.5 \\
244 \\
1.5 \\
10 \\
\multicolumn{2}{c}{.2} \\
245
\end{tabular} & $\begin{array}{c}16 \\
.10 \\
80 \\
4.9 \\
3.6 \\
256 \\
4.3 \\
9 \\
.2 \\
264 \\
220 \\
10\end{array}$ & \begin{tabular}{rr}
14 & \\
\multicolumn{2}{c}{.10} \\
68 & -- \\
2.5 \\
5.5 \\
\multicolumn{2}{c}{} \\
221 & -- \\
1 & \\
7 & \\
0 & \\
221 & --
\end{tabular} & $\begin{array}{c}11 \\
.04 \\
38 \\
2.5 \\
3.4 \\
109 \\
8.8 \\
10 \\
0 \\
149 \\
105 \\
16\end{array}$ \\
\hline 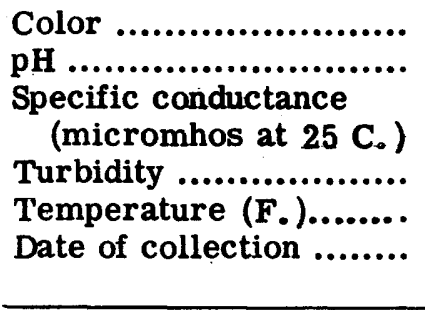 & $\begin{array}{r}7.7 \\
-- \\
-- \\
\text { Aug. } 24, \\
1950\end{array}$ & $\begin{array}{r}7.6 \\
-- \\
-- \\
\text { Aug. } 24, \\
1950\end{array}$ & $\begin{array}{r}7.5 \\
-- \\
-- \\
-- \\
\text { Aug. } 24, \\
1950\end{array}$ & $\begin{array}{r}10 \\
7.5 \\
-- \\
1 \\
-- \\
1949 \\
\end{array}$ & $\begin{array}{r}4 \\
7.8 \\
-- \\
.8 \\
-- \\
\text { May } 21, \\
1949\end{array}$ \\
\hline $\begin{array}{l}\text { Depth (feet) } \ldots . . . . . . . . . . . \\
\text { Diameter (inches)........ } \\
\text { Date drilled ............... } \\
\text { Percent of supply ........ }\end{array}$ & $\begin{array}{r}300 \\
-- \\
1941 \\
--\end{array}$ & $\begin{array}{r}350 \\
-- \\
1930\end{array}$ & $\begin{array}{r}300 \\
-- \\
1943 \\
--\end{array}$ & & \\
\hline
\end{tabular}


ST. PETERSBURG--Continued

Regular determinations at treatment plant, 1951

\begin{tabular}{|c|c|c|c|c|c|c|c|c|c|c|c|c|}
\hline & \multicolumn{3}{|c|}{$\begin{array}{c}\text { Alkalinity } \\
\text { as } \mathrm{CaCO}_{3} \\
\text { (ppm) }\end{array}$} & \multicolumn{3}{|c|}{ pH } & \multicolumn{3}{|c|}{$\begin{array}{c}\text { Hardness } \\
\text { as } \mathrm{CaCO} \mathrm{S}_{3} \\
\text { (ppm) }\end{array}$} & \multicolumn{3}{|c|}{$\begin{array}{c}\text { Tempera- } \\
\text { ture } \\
\left({ }^{\circ} \mathrm{F} .\right)\end{array}$} \\
\hline & $\mathbf{A v}$ & $\operatorname{Max}$ & Min & Av & $\operatorname{Max}$ & Min & $\mathbf{A v}$ & $\operatorname{Max}$ & Min & Av & $\operatorname{Max}$ & Min \\
\hline $\begin{array}{l}\text { Raw water......... } \\
\text { Finished water... }\end{array}$ & $\begin{array}{r}186 \\
88 \\
\end{array}$ & $\begin{array}{l}196 \\
100\end{array}$ & $\begin{array}{l}191 \\
186\end{array}$ & $\begin{array}{l}7.3 \\
7.8\end{array}$ & $\begin{array}{l}7.4 \\
8.0\end{array}$ & $\begin{array}{l}7.2 \\
7.6\end{array}$ & $\begin{array}{r}183 \\
92 \\
\end{array}$ & $\begin{array}{r}192 \\
99 \\
\end{array}$ & $\begin{array}{r}180 \\
88 \\
\end{array}$ & $\begin{array}{l}76 \\
79\end{array}$ & \begin{tabular}{l|}
76 \\
82
\end{tabular} & $\begin{array}{l}76 \\
76\end{array}$ \\
\hline
\end{tabular}

\section{SANFORD}

(Population, 11, 935)

Ownership: Municipal; also supplies about 1,000 people outside the city limits.

Total population supplied, about 12,900 .

Source: 7 wells ( 1 to 7$) 135,80,135,135,135,135$, and $180 \mathrm{ft}$ deep.

Treatment: Aeration, and chlorination.

Raw-water storage: 1, 000, 000 gal.

Finished-water storage: 200,000 gal.

\section{ANALYSES}

(Analyses, in parts per million, by Louis C. Herring \& Co.)

\begin{tabular}{|c|c|c|c|c|}
\hline & Well 1 & Well 3 & Well 6 & $\begin{array}{c}\text { Finished } \\
\text { water a }\end{array}$ \\
\hline 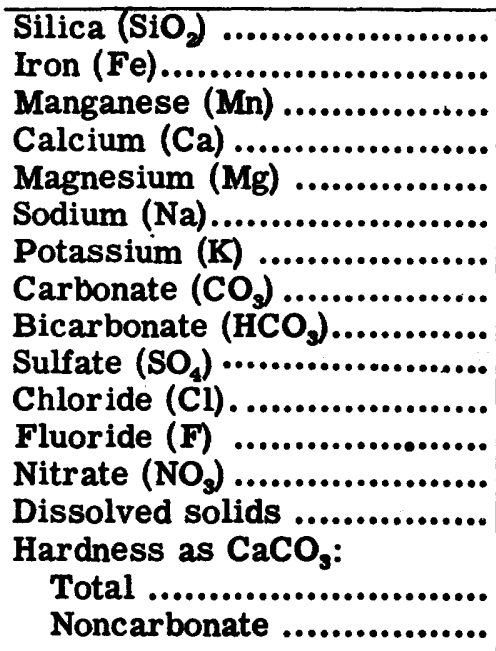 & $\begin{array}{c}7.2 \\
1.6 \\
-- \\
50 \\
9.1 \\
37 \\
180^{--} \\
7.3 \\
62 \\
1.2 \\
275 \\
162 \\
15\end{array}$ & $\begin{array}{c}6.8 \\
1.2 \\
44 \\
9.0 \\
36 \\
168 \\
6.3 \\
56 \\
1.2 \\
245 \\
147 \\
9\end{array}$ & $\begin{array}{c}7.4 \\
1.8 \\
43 \\
8.3 \\
29 \\
{ }^{--} \\
161 \\
6.3 \\
46 \\
1.2^{--} \\
238 \\
141 \\
10\end{array}$ & $\begin{array}{l}10 \\
.24 \\
.00 \\
46 \\
8.7 \\
29 \\
1.6 \\
0 \\
162 \\
7 \\
55 \\
.1 \\
.6 \\
246 \\
151 \\
18\end{array}$ \\
\hline 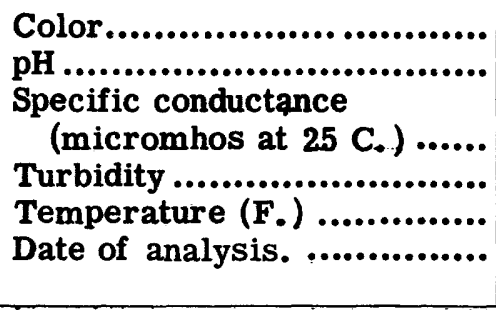 & $\begin{array}{r}7.6 \\
-- \\
-- \\
-- \\
\text { Aug. } 8, \\
1950\end{array}$ & $\begin{array}{r}7.7 \\
-- \\
-- \\
\text { Aug. } 8, \\
1950\end{array}$ & $\begin{array}{r}7 . \overline{7} \\
-- \\
-- \\
-- \\
\text { Aug. } 8, \\
1950\end{array}$ & $\begin{array}{r}2 \\
7.6 \\
430 \\
.3 \\
-- \\
\text { Feb. } 7 \\
1952 \\
\end{array}$ \\
\hline 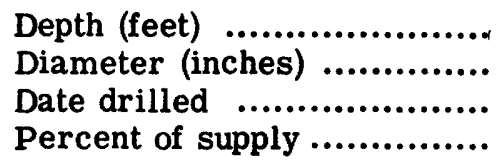 & $\begin{array}{r}135 \\
8 \\
-- \\
--\end{array}$ & $\begin{array}{r}135 \\
8 \\
-- \\
--\end{array}$ & $\begin{array}{r}135 \\
8 \\
-- \\
--\end{array}$ & $\begin{array}{r}80 \text { to } 180 \\
8 \\
--\end{array}$ \\
\hline
\end{tabular}

a Composite, wells 1 to 7 . Analysis by U. S. Feological Survey. 
FLORIDA

SARASOTA

(Population, 18, 896)

Ownership: Municipal.

Source: 16 deep wells in 5 well fields: Plant well field, 5 wells (1 to 5) 640 to $758 \mathrm{ft}$ deep; Northeast well field, 4 wells (1 to 4) each $600 \mathrm{ft}$ deep; Northwest well field, 3 wells (1 to 3) 350,580 , and $570 \mathrm{ft}$ deep; Payne Terminal wells (1 and 2) 529 and $475 \mathrm{ft}$ deep; St. Armands Key wells (1 and 2) 340 and $400 \mathrm{ft}$ deep. The Plant well field is pumped by means of gasoline power and can be used in case of electric power failure.

Treatment: Softening with zeolite (both pressure and gravity type softeners, sea water regenerated).

Rated capacity of treatment plant: 5, 000,000 gpd.

Raw-water storage: 950,000 gal.

Finished-water storage: 1, 935,000 gal.

Partial analyses of samples from all of the wells indicate that the water is of similar chemical composition. The St. Armands Key wells yield water that is lower in dissolved solids and hardness than that from the other wells. The Plant well field yields water with the highest dissolved solids.

\section{ANALYSES}

(Analyses, in parts per million, by Black Laboratory, Inc. ; Gainesville, Fla.)

\begin{tabular}{|c|c|c|c|c|}
\hline & \multicolumn{2}{|c|}{ Northwest well field } & \multirow{2}{*}{ Well $1^{\mathrm{a}}$} & \multirow{2}{*}{$\begin{array}{l}\text { Finished } \\
\text { water }^{b}\end{array}$} \\
\hline & \multicolumn{2}{|l|}{ Well 1} & & \\
\hline \multirow{14}{*}{ 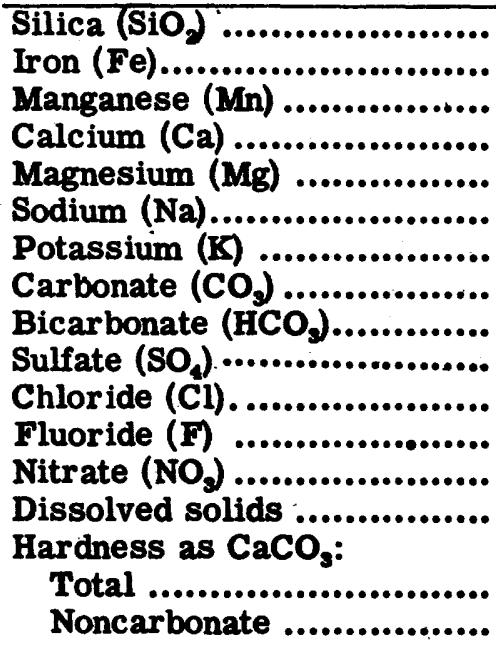 } & 23 & 24 & 30 & 24 \\
\hline & -- & -- & -- & \\
\hline & & -- & -- & \\
\hline & 220 & 196 & 73 & 14 \\
\hline & 100 & 101 & & $530^{\circ}$ \\
\hline & 90 & 37 & 32 & 16 \\
\hline & -- & -- & -- & \\
\hline & 146 & 149 & 193 & 161 \\
\hline & 156 & 180 & 210 & 160 \\
\hline & -- & -- & - & 1.5 \\
\hline & & -- & -- & \\
\hline & c 1,480 & $c_{1}, 500$ & c 573 & $c_{1,650}$ \\
\hline & & & & \\
\hline & $\begin{array}{l}985 \\
866\end{array}$ & $\begin{array}{r}1,110 \\
988\end{array}$ & $\begin{array}{l}359 \\
201\end{array}$ & $\begin{array}{r}36 \\
0\end{array}$ \\
\hline $\begin{array}{l}\text { Color } . \ldots \ldots \ldots \ldots \ldots \ldots \ldots \\
\text { pH } . \ldots \ldots \ldots \ldots \ldots \ldots \ldots \ldots \ldots\end{array}$ & $7 . \overline{5}$ & $7 . \overline{4}$ & $7 . \overline{6}$ & $\begin{array}{r}2 \\
7.5\end{array}$ \\
\hline Specific conductance & & & & \\
\hline (micromhos at 25 C. ) ...... & -- & -- & & 2,420 \\
\hline Turbidity ......................... & & & & \\
\hline Date of analysis & Dec. 11, & Dec, $11^{--}$ & Dec. 11, & Feb. 12, \\
\hline & 1950 & 1950 & 1950 & 1952 \\
\hline Depth (feet) ......... & 350 & 570 & 340 & \\
\hline Diameter (inches) ............... & -- & -- & & \\
\hline Date drilled $\quad . . . . \ldots \ldots \ldots \ldots \ldots \ldots$ & 1950 & 1950 & 1950 & \\
\hline Percent of supply ................ & -- & -- & & \\
\hline
\end{tabular}

a St. Armands Key well field.

b Composite, wells $1,2,3$, and 4, Plant well field; wells 1 and 2 North well

field; well 2, West well. Analysis by U. S. Geological Survey.

c Sum of determined constituents. 
SARASOTA--Continued

Regular determinations at treatment plant, 1951

\begin{tabular}{|c|c|c|c|c|c|c|c|c|c|c|c|c|}
\hline & \multicolumn{3}{|c|}{$\begin{array}{c}\text { Alkalinity } \\
\text { as } \mathrm{CaCO} \\
\text { (ppm) }\end{array}$} & \multicolumn{3}{|c|}{ pH } & \multicolumn{3}{|c|}{$\begin{array}{l}\text { Hardness } \\
\text { as } \mathrm{CaCO}_{3} \\
(\mathrm{ppm})\end{array}$} & \multicolumn{3}{|c|}{ Turbidity } \\
\hline & Av & $\operatorname{Max}$ & Min & Av & $\operatorname{Max}$ & Min & Av & Max & Min & Av & $\operatorname{Max}$ & Min \\
\hline $\begin{array}{l}\text { Raw water......... } \\
\text { Finished water... }\end{array}$ & $\begin{array}{l}140 \\
140 \\
\end{array}$ & $\begin{array}{l}145 \\
150\end{array}$ & $\begin{array}{l}136 \\
136 \\
\end{array}$ & $\begin{array}{l}7.8 \\
8.0 \\
\end{array}$ & $\begin{array}{l}7.9 \\
8.2 \\
\end{array}$ & $\begin{array}{l}7.6 \\
7.9\end{array}$ & $\begin{array}{r}1060 \\
45 \\
\end{array}$ & $\begin{array}{r}1120 \\
55 \\
\end{array}$ & $\begin{array}{r}985 \\
36 \\
\end{array}$ & $\begin{array}{l}-- \\
--\end{array}$ & -- & $=-$ \\
\hline
\end{tabular}

TALLAHASSEE

(Population, 27, 237)

Ownership: Municipal; supplies also about 7,500 people outside the city limits.

Source: 9 wells ( 1 to 9 ). Wells 1 and 2 are obsolete; 3 and 4 , for emergency use only. The depths of wells 5 to 9 are reported to be 246, 414, 400, 427, and $350 \mathrm{ft}$, respectively. (Wells 10 and 11 were under construction at the time of obtaining well data).

Treatment: Chlorination and fluoridation. a

Storage: 1 ground reservoir, 400,000 gal; 2 elevated tanks, 400,000 and 500,000 gal.

\section{ANALYSES}

(Analyses, in parts per million, by U. S. Geological Survey)

\begin{tabular}{|c|c|c|c|c|c|}
\hline & $\begin{array}{l}\text { Wells } \\
8 \text { and } 9 b\end{array}$ & Well 9c & & $\begin{array}{c}\text { Wells } \\
8 \text { and } 9 b\end{array} \mid$ & Well $9 \mathrm{c}$ \\
\hline $\begin{array}{l}\text { Silica }\left(\mathrm{SiO}_{2}\right) \\
\text { Iron }(\mathrm{Fe}) \ldots \ldots \ldots \ldots \\
\text { Manganese }(\mathrm{Mn}) \ldots \ldots\end{array}$ & $\begin{array}{r}14 \\
.00 \\
.00\end{array}$ & $\begin{array}{r}8 \\
.02 \\
.00\end{array}$ & 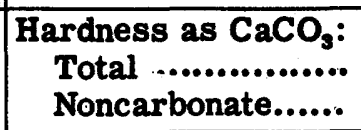 & $\begin{array}{r}152 \\
10\end{array}$ & $\begin{array}{r}124 \\
4\end{array}$ \\
\hline $\begin{array}{l}\text { Calcium }(\mathrm{Ca}) \ldots \ldots \\
\text { Magnesium }(\mathrm{Mg}) \ldots . \\
\text { Sodium }(\mathrm{Na}) \ldots \ldots \\
\text { Potassium }(\mathrm{K}) \\
\text { Carbonate }\left(\mathrm{CO}_{3}\right) \ldots \ldots \\
\text { Bicarbonate }\left(\mathrm{HCO}_{3}\right) \\
\text { Sulfate }\left(\mathrm{SO}_{4}\right) \ldots \ldots \\
\text { Chloride }(\mathrm{Cl}) \\
\text { Fluoride }(\mathrm{F}) \\
\text { Nitrate }(\mathrm{NO} \\
\text { Dissolved }\end{array}$ & $\begin{array}{c}41 \\
12 \\
3.0 \\
.2 \\
0 \\
173 \\
6.5 \\
5.4 \\
.1 \\
.9 \\
164\end{array}$ & $\begin{array}{c}36 \\
8.3 \\
7.8 \\
146 \\
5.4 \\
10 \\
1.8 \\
173\end{array}$ & $\begin{array}{l}\text { Color ................... } \\
\text { pH ........................ } \\
\text { Specific conductance } \\
\text { (micromhos at } \\
25 \text { C.) ............... } \\
\text { Turbidity .............. } \\
\text { Temperature (F.)... } \\
\text { Date of collection... }\end{array}$ & $\begin{array}{r}2 \\
7.8 \\
\\
287 \\
2.4 \\
-- \\
\text { Jan. 4, } \\
1952\end{array}$ & $\begin{array}{r}10 \\
7.8 \\
0 \\
-- \\
--\end{array}$ \\
\hline $\begin{array}{l}\text { Depth (feet) } \\
\text { Diameter (inches) } \\
\text { Date drilled ........ } \\
\text { Percent of supply }\end{array}$ & & & & $\begin{array}{r}427,350 \\
-- \\
1946 \\
--\end{array}$ & $\begin{array}{r}350 \\
-- \\
1946 \\
--\end{array}$ \\
\hline
\end{tabular}

a Fluoridation was begun about Mar. 1, 1952

b Composite. Collected from distribution system. Not fluoridated.

c Analysis by Pittsburgh Testing Laboratory, Pittsburgh, Pa. 
TAMPA

(Population, 124, 681)

Ownership: Municipal; supplies also about 50,000 people outside the city limits. Total population supplied, about 175,000 .

Source: Hillsborough River, 97 percent of supply; wells, 3 percent.

Treatment: The treatment varies from mainly color removal with alum during wet seasons to lime softening during dry seasons when the use of well water increases and the hardness of the river water increases.

Rated capacity of treatment plant: $30,000,000 \mathrm{gpd}$.

Raw-water storage: None.

Finished-water storage: 5, 200, 000 gal.

The composition of the river water varies considerably throughout the year.

\section{ANALYSES}

(Analyses, in parts per million, by U. S. Geological Survey)

\begin{tabular}{|c|c|c|c|c|c|}
\hline & $\begin{array}{l}\text { Finished } \\
\text { water }\end{array}$ & $\begin{array}{c}\text { Finished } \\
\text { water }\end{array}$ & & $\begin{array}{c}\text { Finished } \\
\text { water }\end{array}$ & $\begin{array}{l}\text { Finished } \\
\text { water }\end{array}$ \\
\hline $\begin{array}{l}\text { Silica }\left(\mathrm{SiO}_{2}\right) \\
\text { Iron }(\mathrm{Fe}) \ldots \ldots \ldots \ldots \\
\text { Manganese }(\mathrm{Mn}) \ldots \ldots . . . .\end{array}$ & $\begin{array}{l}5.3 \\
.02 \\
.0\end{array}$ & $\begin{array}{l}- \\
.15 \\
--\end{array}$ & $\begin{array}{r}\text { Hardiness as } \mathrm{CaCO}_{3}: \\
\text { Total .................. } \\
\text { Noncarbonate...... }\end{array}$ & $\begin{array}{l}91 \\
34\end{array}$ & $\begin{array}{l}72 \\
43\end{array}$ \\
\hline $\begin{array}{l}\text { Calcium (Ca) .......... } \\
\text { Magnesium (Mg)..... }\end{array}$ & $\begin{array}{r}29 \\
4.5\end{array}$ & & Color...................... & 2 & 30 \\
\hline $\begin{array}{l}\text { Sodium (Na) .... } \\
\text { Potassium (K) }\end{array}$ & 4.9 & 7.7 & pH ....................... & 7.5 & 7.5 \\
\hline $\begin{array}{l}\text { Carbonate }\left(\mathrm{CO}_{3}\right) \ldots . . . \\
\text { Bicarbonate }\left(\mathrm{HCO}_{3}\right)\end{array}$ & $\begin{array}{r}0 \\
69\end{array}$ & $\begin{array}{r}0 \\
35\end{array}$ & 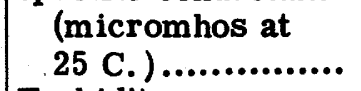 & 203 & 192 \\
\hline Sulfate $\left(\mathrm{SO}_{4}\right) \ldots \ldots \ldots$ & 21 & 37 & Turbidity ............... & -- & -- \\
\hline Chloride (Cl) .......... & 16 & & Temperature (F.)... & & \\
\hline Fluoride $(\mathrm{F}) \ldots \ldots \ldots$ & $\begin{array}{r}.1 \\
.4\end{array}$ & $\begin{array}{l}.2 \\
.3\end{array}$ & Date of collection... & $\begin{array}{c}\text { Mar. 11, } \\
1949\end{array}$ & $\begin{array}{c}\text { Nov. } 9 \\
1951\end{array}$ \\
\hline Dissolved solids..... & 123 & & & & \\
\hline
\end{tabular}

Regular determinations at treatment plant, 1950-1951

\begin{tabular}{|c|c|c|c|c|c|c|c|c|c|c|c|c|}
\hline & \multicolumn{3}{|c|}{$\begin{array}{c}\text { Alkalinity } \\
\text { as } \mathrm{CaCO} \\
\text { (ppm) }\end{array}$} & \multicolumn{3}{|c|}{ pH } & \multicolumn{3}{|c|}{$\begin{array}{c}\text { Hardness } \\
\text { as } \mathrm{CaCO} \\
\text { (ppm) }\end{array}$} & \multicolumn{3}{|c|}{ Color } \\
\hline & Av & Max & Min & Av & $\operatorname{Max}$ & Min & Av & $\operatorname{Max}$ & Min & Av & $\operatorname{Max}$ & Min \\
\hline Raw water.......... & 89 & 130 & 46 & 7.3 & 8.3 & 6.8 & 111 & 144 & 60 & 96 & 190 & 30 \\
\hline Finished water... & 59 & 96 & 34 & 8.3 & 8.7 & 7.8 & 115 & 160 & 58 & 16 & 25 & 8 \\
\hline
\end{tabular}




\section{WEST PALM BEACH \\ (Population, 43, 162)}

Ownership: West Palm Beach Water Company; supplies also Palm Beach. Total population supplied, about 47,000 .

Source: Clear Lake and Lake Mangonea (Loxahatchee Marsh).

Treatment: Aeration, coagulation with alum, sedimentation, rapid sand filtration, chlorination, and adjustment of $\mathrm{pH}$ with lime.

Rated capacity of treatment plant: 26,000,000 gpd.

Raw-water storage: 2, 100,000,000 gal.

Finished-water storage: 4,000,000 gal.

At certain periods of the year, well water having an alkalinity of $120 \mathrm{ppm}$ is added to the raw-water in amounts varying between 3 to 10 percent of the total to increase the alkalinity of the raw water. The wells are considered as an adjunct to the treatment plant rather than as an auxiliary supply.

\section{ANALYSES}

(Analyses, in parts per million, by So. Analytical Laboratory, Jacksonville)

\begin{tabular}{|c|c|c|c|}
\hline & $\begin{array}{c}\text { Raw } \\
\text { water }\end{array}$ & $\begin{array}{l}\text { Finished } \\
\text { water }\end{array}$ & $\begin{array}{r}\text { Finished } \\
\text { water a }\end{array}$ \\
\hline 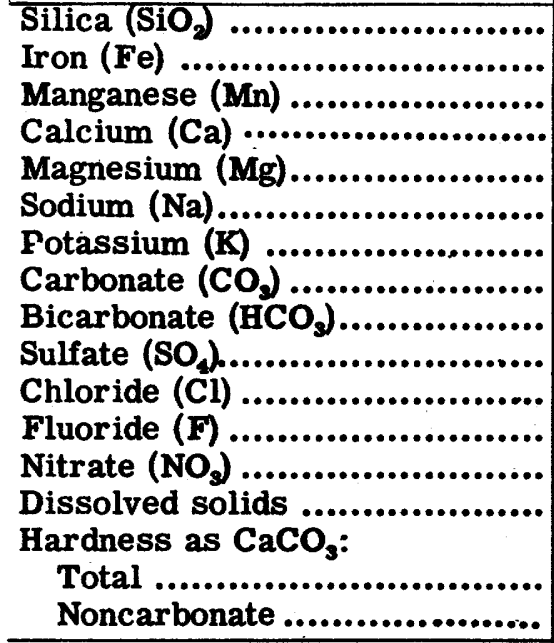 & 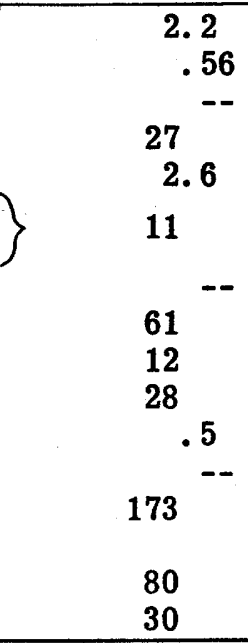 & 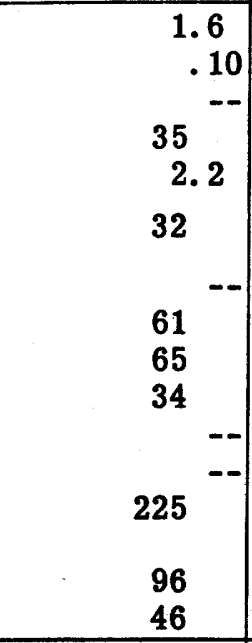 & $\begin{array}{r}3.7 \\
3 .- \\
0 \\
26 \\
23 \\
16 \\
.1 \\
.3 \\
\quad-- \\
\quad \\
60 \\
39 \\
\end{array}$ \\
\hline 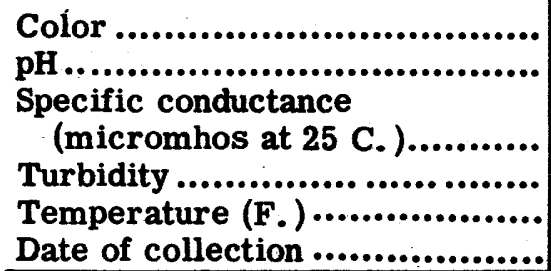 & $\begin{array}{r}25 \\
7.7 \\
-- \\
18 \\
\text { June } 21,19 \overline{4}\end{array}$ & $\begin{array}{r}7.9 \\
-- \\
5 \\
\text { June } 21,1949\end{array}$ & $\begin{array}{r}5 \\
8.1 \\
155 \\
-- \\
\text { Nov. } 21,195 \overline{1}\end{array}$ \\
\hline
\end{tabular}

Regular determinations at treatment plant, 1950

\begin{tabular}{|c|c|c|c|c|c|c|c|c|c|c|c|c|}
\hline & \multicolumn{3}{|c|}{$\begin{array}{c}\text { Alkalinity } \\
\text { as } \mathrm{CaCO} \\
\text { (ppm) }\end{array}$} & \multicolumn{3}{|c|}{ pH } & \multicolumn{3}{|c|}{$\begin{array}{l}\text { Hardness } \\
\text { as CaCO, } \\
\text { (ppm) }\end{array}$} & \multicolumn{3}{|c|}{ Color } \\
\hline & Av & Max & Min & Av & Max & Min & Av & Max & Min & Av & $\operatorname{Max}$ & Min \\
\hline $\begin{array}{l}\text { Raw water......... } \\
\text { Finished water... }\end{array}$ & $\begin{array}{l}34 \\
22\end{array}$ & \begin{tabular}{|l|}
66 \\
40
\end{tabular} & $\begin{array}{l}16 \\
14\end{array}$ & $\begin{array}{l}7.6 \\
8.5\end{array}$ & \begin{tabular}{|l|}
8.5 \\
9.7 \\
\end{tabular} & $\begin{array}{l}6.8 \\
7.1\end{array}$ & $\begin{array}{l}56 \\
76\end{array}$ & $\begin{array}{l}82 \\
93 \\
\end{array}$ & $\begin{array}{l}29 \\
49\end{array}$ & $\begin{array}{r}71 \\
6\end{array}$ & $\begin{array}{l}95 \\
20 \\
\end{array}$ & $\begin{array}{r}35 \\
3\end{array}$ \\
\hline
\end{tabular}

${ }^{a}$ Analysis by U. S. Geological Survey. 


\section{ALBANY \\ (Population, 31, 155)}

Ownership: Municipal; also supplies suburban areas and Turner Air Force Base. Total population supplied, about 37,000 .

Source: 7 deep wells (6 to 12). (Wells 7 and 8 under repair and not in use as of Feb. 2, 1952). The depths of wells 6, 9, 10, 11, 12 are 955, 795, 868, 915, and $725 \mathrm{ft}$, respectively; yields reported to be $1,500,1,669,1,248,1,250$ and $1,175 \mathrm{gpm}$.

Treatment: Chlorination at wells.

Raw-water storage: None.

Finished-water storage: 3 reservoirs, 310,000, 244,000, and 998, 000 gal; 2 elevated tanks, 500,000 gal each.

\section{ANALYSES}

(Analyses, in parts per million, by U. S. Geological Survey)

\begin{tabular}{|c|c|c|c|}
\hline & $\begin{array}{c}\text { Well } 11 \\
\text { (raw water) }\end{array}$ & $\begin{array}{l}\text { Finished } \\
\text { water a }\end{array}$ & $\begin{array}{c}\text { Well } 9 \\
\text { (finished water) }\end{array}$ \\
\hline 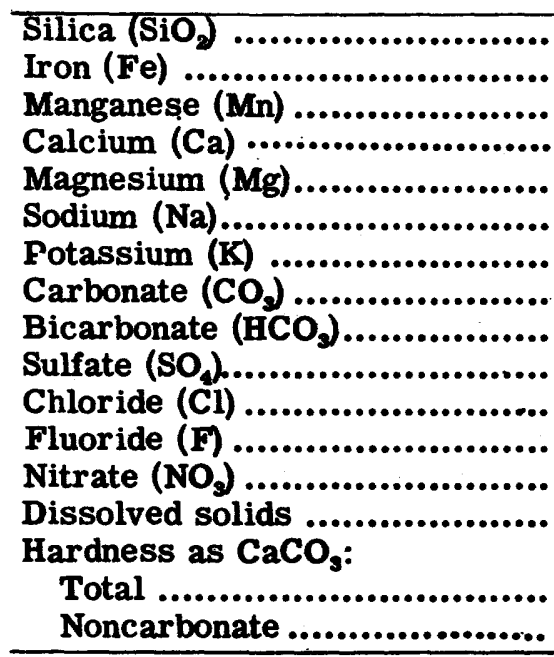 & $\begin{array}{c}26 \\
.11 \\
26 \\
.00 \\
7.7 \\
28 \\
0 \\
173 \\
11 \\
2.9 \\
.2 \\
.3 \\
188 \\
96 \\
0\end{array}$ & $\begin{array}{c}26 \\
.10 \\
30 \\
6.5 \\
33 \\
0 \\
191 \\
10 \\
4.4 \\
.3 \\
.2 \\
202 \\
102 \\
0\end{array}$ & $\begin{array}{c}32.11 \\
32.00 \\
6.5 \\
24 \\
0 \\
176 \\
8.6 \\
3.2 \\
.2 \\
.2 \\
194 \\
107 \\
0\end{array}$ \\
\hline 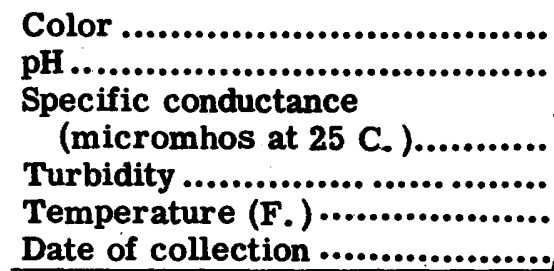 & \begin{tabular}{r|}
2 \\
8.0 \\
295 \\
0 \\
73 \\
Dec. 1,1951
\end{tabular} & $\begin{array}{r}7.8 \\
7.8 \\
325 \\
0 \\
69 \\
\text { Dec. } 1,1951\end{array}$ & $\begin{array}{r}1 \\
7.8 \\
297 \\
1 \\
72 \\
\text { Dec. } 1,1951\end{array}$ \\
\hline 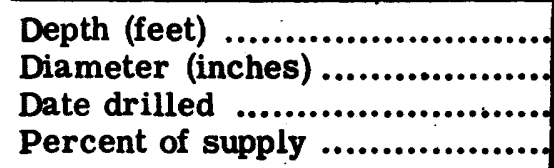 & $\begin{array}{r}915 \\
20 \\
1950 \\
--\end{array}$ & & $\begin{array}{r}795 \\
20 \\
1947 \\
--\end{array}$ \\
\hline
\end{tabular}

a Mixed water from wells 7,8 , and 10. 


\section{AMERICUS \\ (Population, 11, 389)}

Ownership: Municipal; also supplies suburban districts. Total population supplied, about 11,400 .

Source: 5 wells ( 1 to 5 ) $259,168,635,635$, and $450 \mathrm{ft}$ deep.

Treatment: Chlorination.

Raw-water storage: None.

Finished-water storage: 4 reservoirs, 422,000 gal; elevated tank, 350,000 gal.

\section{ANALYSES}

(Analyses, in parts per million, by U. S. Geological Survey)

\begin{tabular}{|c|c|c|c|}
\hline & Well 1 & Well 2 & $\begin{array}{c}\text { Well } 4 \\
\text { (finished water) }\end{array}$ \\
\hline 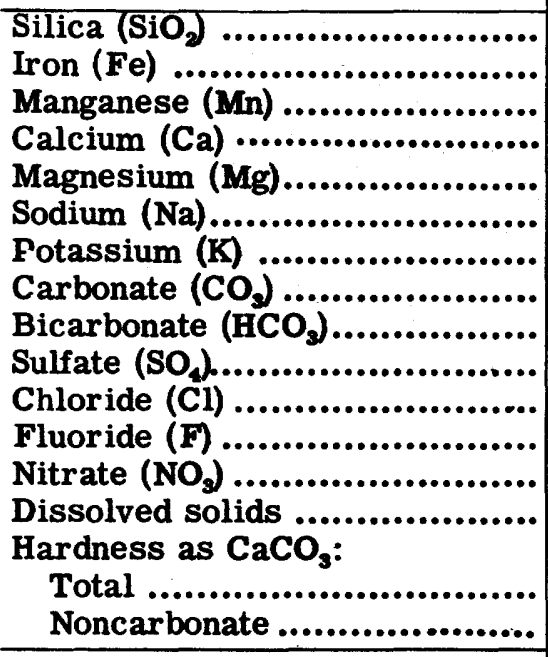 & $\begin{array}{c}28 \\
.18 \\
46 \\
4.5 \\
4.5 \\
3.4 \\
3.4 \\
0 \\
152 \\
16 \\
2.0 \\
.0 \\
.1 \\
177 \\
133 \\
9\end{array}$ & $\begin{array}{c}50 \\
.36 \\
30 \\
30 \\
3.1 \\
3.3 \\
3.0 \\
0 \\
96 \\
14 \\
2.0 \\
.0 \\
.1 \\
155 \\
88 \\
9\end{array}$ & $\begin{array}{c}62 \\
.38 \\
.03 \\
27 \\
3.0 \\
2.0 \\
0 \\
85 \\
9.2 \\
3.2 \\
.1 \\
.1 \\
154 \\
80 \\
10\end{array}$ \\
\hline 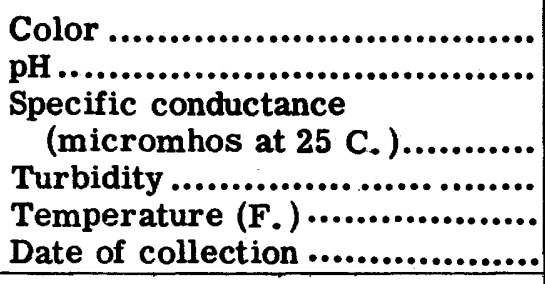 & $\begin{array}{r}-- \\
- \\
-- \\
-- \\
67 \\
\text { Jan. } 21,1938 \\
\end{array}$ & $\begin{array}{r}-- \\
-- \\
-- \\
-- \\
-- \\
\text { Jan. } 21,1938 \\
\end{array}$ & $\begin{array}{r}7.7 \\
169 \\
0 \\
72 \\
\text { Dec. } 1,1951 \\
\end{array}$ \\
\hline 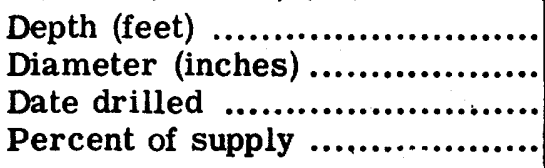 & $\begin{array}{r}259 \\
12 \\
1927 \\
--\end{array}$ & $\begin{array}{r}168 \\
10 \\
1914 \\
--\end{array}$ & $\begin{array}{r}635 \\
12 \\
1947 \\
--\end{array}$ \\
\hline
\end{tabular}




\section{ATHENS}

(Population, 28, 180)

Ownership: Municipal; also supplies suburban districts. Total population supplied, about 32,000 .

Source: Oconee River and/or Sandy Creek.

Treatment: Prechlorination, ammoniation, coagulation with alum and lime, carbon for taste and odor control, sedimentation, fluoridation, rapid sand filtration, and adjustment of $\mathrm{pH}$ with lime.

Rated capacity of treatment plant: 3,000,000 gpd.

Raw-water storage: Reservoir, 100,000,000 gal.

Finished-water storage: 2 clear wells, 500,000 and 750,000 gal; 2 elevated tanks, 750,000 and 250,000 gal.

The intake on the Oconee River is adjacent to the treatment plant in the north section of the city. Water is pumped from the river directly to the treatment plant. The intake on Sandy Creek is about $\frac{1}{2}$ mile north of the city. Normally, creek water is pumped to a 100,000,000 gal raw-water reservoir, thence to the treatment plant.

ANALYSES

(Analyses, in parts per million, by U. S. Geological Survey)

\begin{tabular}{|c|c|c|c|c|}
\hline & $\begin{array}{c}\text { Oconee } \\
\text { River } \\
\text { (raw water) }\end{array}$ & $\begin{array}{c}\text { Sandy } \\
\text { Creek } \\
\text { (raw water) }\end{array}$ & $\begin{array}{l}\text { Finished } \\
\text { water a }\end{array}$ & $\begin{array}{l}\text { Finished } \\
\text { water b }\end{array}$ \\
\hline Silica $\left(\mathbf{S i O}_{2}\right) \ldots \ldots \ldots \ldots \ldots \ldots \ldots$ & 15 & 14 & 12 & 14 \\
\hline Iron $(\mathrm{Fe}) \ldots . . . \ldots \ldots \ldots \ldots \ldots . . . . . .$. & .05 & .05 & .10 & .06 \\
\hline Manganese $(\mathrm{Mn})$................. & -- & .00 & .00 & .03 \\
\hline Calcium (Ca) ..................... & 3.9 & 3.6 & 8.8 & 13 \\
\hline Magnesium (Mg) ................ & 1.8 & 1.3 & 1.4 & 1.6 \\
\hline Sodium $(\mathrm{Na}) \ldots \ldots \ldots \ldots \ldots \ldots \ldots$ & 3.8 & 4.2 & 3.9 & 4.4 \\
\hline 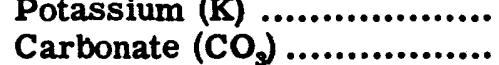 & 0 & 0 & 0 & 0 \\
\hline Bicarbonate $\left(\mathrm{HCO}_{3}\right) . . . . . . . . . . . .$. & 25 & 21 & 22 & 30 \\
\hline Sulfate $\left(\mathrm{SO}_{4}\right) \ldots \ldots \ldots \ldots \ldots \ldots \ldots$ & 2.9 & 2.1 & 13 & 17 \\
\hline Chloride (C1) ...................... & 2.1 & 2.4 & 3.0 & 3.2 \\
\hline Fluoride $(F)$.................... & .1 & .1 & .0 & .6 \\
\hline Nitrate $\left(\mathrm{NO}_{s}\right), \ldots . . . \ldots \ldots \ldots \ldots . . . . . .$. & .3 & .5 & .4 & $70^{.4}$ \\
\hline Dissolved solids ................. & 43 & 40 & 55 & 73 \\
\hline Hardness as $\mathrm{CaCO}_{3}$ : & 17 & 14 & 28 & 39 \\
\hline 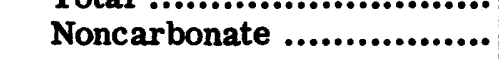 & 0 & 0 & 10 & 14 \\
\hline Color................................ & 6 & 3 & 2 & \\
\hline 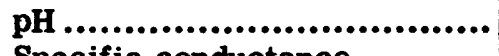 & -- & 6.6 & 6.8 & 8.2 \\
\hline $\begin{array}{l}\text { specinc conauctance } \\
\text { (micromhos at } 25 \mathrm{C}_{\text {. }} \text { )...... }\end{array}$ & -- & 42.6 & 79.4 & 107 \\
\hline Turbidity..................... & -- & -- & & -- \\
\hline Temperature (F.) ............... & & & & - \\
\hline Date of collection ................ & $\begin{array}{c}\text { Nov. } 15, \\
1940\end{array}$ & $\underset{1951}{\operatorname{Mar}_{.1} 1,}$ & $\underset{1951}{\operatorname{Mar} .1}$ & $\begin{array}{c}\text { July } 31, \\
1951\end{array}$ \\
\hline
\end{tabular}

Regular determinations at treatment plant, 1950

\begin{tabular}{|c|c|c|c|c|c|c|c|c|c|c|c|c|}
\hline & \multicolumn{3}{|c|}{$\begin{array}{c}\text { Alkalinity } \\
\text { as CaCO, } \\
\text { (ppm) }\end{array}$} & \multicolumn{3}{|c|}{ pH } & \multicolumn{3}{|c|}{$\begin{array}{c}\text { Hardness } \\
\text { as } \mathrm{CaCO}_{3} \\
\text { (ppm) }\end{array}$} & \multicolumn{3}{|c|}{ Turbidity } \\
\hline & Av & $\operatorname{Max}$ & Min & Av & Max & Min & Av & $\operatorname{Max}$ & Min & Av & $\operatorname{Max}$ & Min \\
\hline $\begin{array}{l}\text { Raw water.......... } \\
\text { Finished water... }\end{array}$ & $\begin{array}{l}14 \\
20\end{array}$ & $\begin{array}{l}18 \\
26\end{array}$ & $\begin{array}{l}10 \\
15\end{array}$ & $\begin{array}{l}6.6 \\
8.3\end{array}$ & \begin{tabular}{|l|}
6.7 \\
8.3 \\
\end{tabular} & $\begin{array}{l}6.5 \\
8.2\end{array}$ & $\begin{array}{l}-- \\
--\end{array}$ & $\begin{array}{l}-- \\
--\end{array}$ & - & $\begin{array}{r}20 \\
0\end{array}$ & $\begin{array}{r}25 \\
0\end{array}$ & $\begin{array}{r}15 \\
0\end{array}$ \\
\hline
\end{tabular}

a Sandy Creek.

b Mixed sample, Sandy Creek and Oconee River. 


\section{ATLANTA}

(Population, 331, 314)

Ownership: Municipal; also supplies suburban areas, the cities of Forest Park, Hapeville, Marietta, Smyrna, and communities in Cobb, DeKalb, and Fulton counties. Total population supplied, about 550,000.

Source: Chattahoochee River.

Treatment: Coagulation with alum and bleaching clay, chlorination, ammoniation, activated carbon, sedimentation, rapid sand filtration, and adjustment of $\mathrm{pH}$ with lime. Copper sulphate is used for the control of algae.

Rated capacity of treatment plant: $70,000,000$ gpd.

Raw-water storage: Reservoirs, 500,000,000 gal.

Finished-water storage: Clear water well, 10,000,000 gal; standpipe, 500,000 gal; elevated tank, 2, 000, 000 gal.

The intake is about 7 miles northwest of the center of the city and about 4 miles from the treatment plant. The water is pumped from the river to the raw-water reservoirs from which it flows to the treatment plant. The finished water is pumped into the distribution system and to elevated storage.

\section{ANALYSES}

(Analyses, in parts per million, by U. S. Geological Survey)

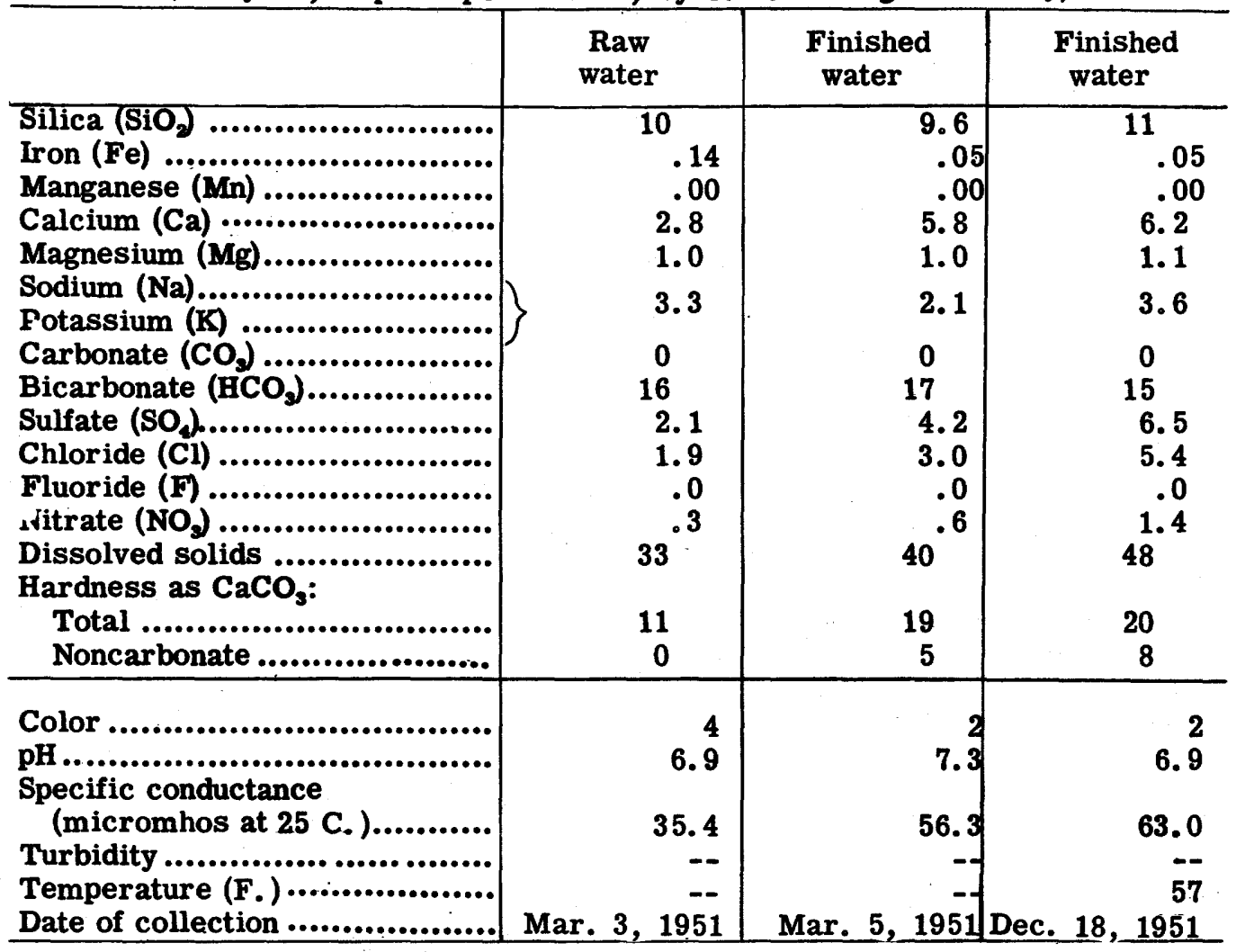

Regular determinations at treatment plant, 1950

\begin{tabular}{|c|c|c|c|c|c|c|c|c|c|c|c|c|}
\hline & \multicolumn{3}{|c|}{$\begin{array}{c}\text { Alkalinity } \\
\text { as CaCO, } \\
\text { (ppm) }\end{array}$} & \multicolumn{3}{|c|}{ pH } & \multicolumn{3}{|c|}{$\begin{array}{l}\text { Hardness } \\
\text { as CaCO, } \\
\text { (ppm) }\end{array}$} & \multicolumn{3}{|c|}{ Turbidity } \\
\hline & $\mathrm{AV}$ & $\operatorname{Max}$ & Min & $\mathbf{A V}$ & Max & Min & $A \nabla$ & $\operatorname{Max}$ & Min & Av & $\operatorname{Max}$ & Min \\
\hline $\begin{array}{l}\text { Raw water......... } \\
\text { Finished' water... }\end{array}$ & $\begin{array}{l}12 \\
16\end{array}$ & $\begin{array}{l}14 \\
17\end{array}$ & $\begin{array}{l}10 \\
14\end{array}$ & $\begin{array}{l}6.9 \\
8.7\end{array}$ & \begin{tabular}{|l|}
7.2 \\
8.9
\end{tabular} & $\begin{array}{l}6.5 \\
8.5\end{array}$ & $\begin{array}{l}10 \\
19\end{array}$ & $\begin{array}{l}11 \\
23\end{array}$ & $\begin{array}{r}8 \\
18\end{array}$ & $\begin{array}{r}26 \\
.10\end{array}$ & $\begin{array}{l}150 \\
.30\end{array}$ & $\begin{array}{r}7 \\
.10\end{array}$ \\
\hline
\end{tabular}




\section{AUGUSTA}

(Population, 71, 508)

Ownership: Municipal; also supplies suburban areas. Total population supplied, about 110,000 .

Source: Savannah River. The intake is 3 miles north of the city.

Treatment: Coagulation with alum, sedimentation, rapid sand filtration, chlorination, and adjustment of $\mathrm{pH}$ with lime.

Rated capacity of treatment plant: $21,000,000 \mathrm{gpd}$.

Raw-water storage: 2 reservoirs, 60,000,000 gal each.

Finished-water storage: 2 clear wells, 3,000,000 and 1,000,000 gal; elevated tank, 500,000 gal; standpipe, 250,000 gal.

\section{ANALYSES}

(Analyses, in parts per million, by U. S. Geological Survey)

\begin{tabular}{|c|c|c|c|c|c|}
\hline & $\begin{array}{l}\text { Raw } \\
\text { water }\end{array}$ & $\begin{array}{c}\text { Finished } \\
\text { water }\end{array}$ & & $\begin{array}{c}\text { Raw } \\
\text { water }\end{array}$ & $\begin{array}{c}\text { Finished } \\
\text { water }\end{array}$ \\
\hline $\begin{array}{l}\text { Silica }\left(\mathrm{SiO}_{2}\right) \\
\text { Iron }(\mathrm{Fe}) \ldots \ldots \ldots \ldots \\
\text { Manganese }(\mathrm{Mn}) \ldots \ldots . . . . .\end{array}$ & $\begin{array}{r}12 \\
.10 \\
.00\end{array}$ & $\begin{array}{r}12 \\
.03 \\
.00\end{array}$ & $\begin{array}{r}\text { Hardness as } \mathrm{CaCO}_{3} \text { : } \\
\text { Total } \ldots . . . . . . . . . . . . . \\
\text { Noncarbonate...... }\end{array}$ & $\begin{array}{r}13 \\
0\end{array}$ & $\begin{array}{r}20 \\
5\end{array}$ \\
\hline $\begin{array}{l}\text { Calcium }(\mathrm{Ca}) . . . . . . . . \\
\text { Magnesium }(\mathrm{Mg}) . . . .\end{array}$ & $\begin{array}{l}3.0 \\
1.3\end{array}$ & 1.3 & Color....................... & 8 & 3 \\
\hline $\begin{array}{l}\text { Sodium (Na) ........... } \\
\text { Potassium (K) ....... }\end{array}$ & 3.6 & 4.0 & $\begin{array}{l}\text { pH ......................... } \\
\text { Specific conductance }\end{array}$ & 6.9 & 7. 0 \\
\hline $\begin{array}{l}\text { Carbonate }\left(\mathrm{CO}_{3}\right) \ldots . . . \\
\text { Bicarbonate }(\mathrm{HCO})\end{array}$ & $\begin{array}{r}0 \\
17\end{array}$ & $\begin{array}{r}0 \\
18\end{array}$ & (micromhos at & 44.2 & 62.7 \\
\hline Sulfate $\left(\mathrm{SO}_{4}\right) \ldots . . . . .$. & 2.8 & 8.8 & Turbidity ............... & - & - \\
\hline Chloride (Cl) . & 2.6 & 3.1 & Temperature (F.)... & $\quad--$ & \\
\hline $\begin{array}{l}\text { Fluoride }(\mathrm{F}) \ldots \ldots \ldots \\
\text { Nitrate }\left(\mathrm{NO}_{\mathrm{g}}\right) \ldots \ldots \ldots \\
\text { Dissolved } \ldots \ldots . . . .\end{array}$ & $\begin{array}{r}.0 \\
.3\end{array}$ & $\begin{array}{r}.0 \\
.3\end{array}$ & Date of collection... & $\begin{array}{l}\text { Apr. 7, } \\
1951\end{array}$ & $\begin{array}{c}\text { Apr. } 7, \\
1951\end{array}$ \\
\hline & & & & & \\
\hline
\end{tabular}

Regular determinations at treatment plant, 1950

\begin{tabular}{l|r|r|r|r|r|r|r|r|r|r|r|r}
\hline & \multicolumn{3}{|c|}{$\begin{array}{c}\text { Alkalinity } \\
\text { as CaCO } \\
\text { (ppm) }\end{array}$} & \multicolumn{3}{c|}{ pH } & \multicolumn{3}{c|}{$\begin{array}{c}\text { Hardness } \\
\text { as CaCO } \\
\text { (ppm) }\end{array}$} & \multicolumn{3}{c}{ Turbidity } \\
\cline { 2 - 15 } & Av & Max & Min & Av & Max & Min & Av & Max & Min & Av & Max & Min \\
\hline Raw water......... & 16 & 17 & 15 & 7.2 & 7.3 & 7.0 & -- & - & -- & 66 & 150 & 25 \\
Finished water... & 15 & 16 & 14 & 7.5 & 7.2 & 7.2 & -- & -- & -- & 1 & 1 & 1 \\
\hline
\end{tabular}


BRUNSWICK

(Population, 17, 954)

Ownership: Peoples Water Service Company of Georgia; also supplies suburban areas. Total population supplied, about 22,000.

Source: 4 wells (1 to 4) 1,027, 1,000,1,000, and $750 \mathrm{ft}$ deep. The wells flow, but wells 1,3 , and 4 are equipped with turbine pumps.

Treatment: Chlorination.

Raw-water storage: 3 reservoirs, 200,000, 100,000, and 100,000 gal.

Finished-water storage: None.

\section{ANALYSES}

(Analyses, in parts per million, by U. S. Geological Survey)

\begin{tabular}{|c|c|c|c|}
\hline & $\begin{array}{c}\text { Well } 3 \\
\text { (raw water) }\end{array}$ & $\begin{array}{c}\text { Well } 4 \\
\text { (raw water) }\end{array}$ & $\begin{array}{c}\text { Wells } 1 \text { and } 2 \\
\text { (finished } \\
\text { water) }\end{array}$ \\
\hline 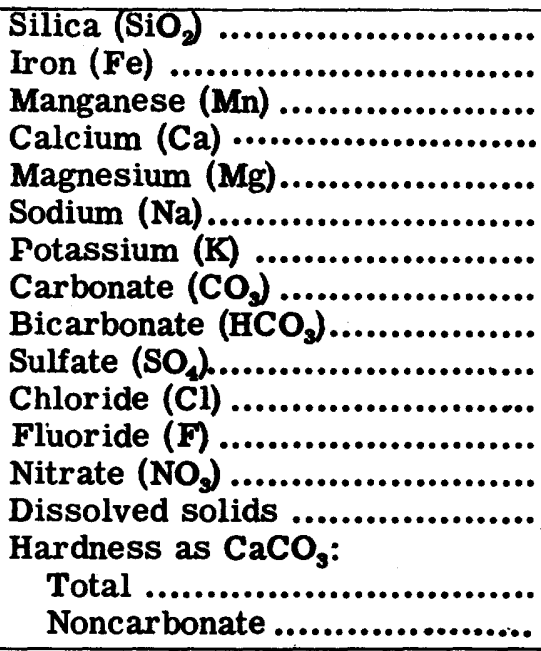 & $\begin{array}{c}36 \\
.18 \\
.00 \\
39 \\
24 \\
12 \\
0 \\
143 \\
78 \\
15 \\
.6 \\
.2 \\
283 \\
196 \\
79 \\
\end{array}$ & $\begin{array}{c}37 \\
.10 \\
40 \\
40 \\
24 \\
12 \\
0 \\
143 \\
79 \\
17 \\
.5 \\
.0 \\
290 \\
198 \\
81 \\
\end{array}$ & $\begin{array}{r}41 \\
.06 \\
102 \\
67 \\
108 \\
0 \\
142 \\
376 \\
181 \\
.7 \\
.2 \\
1,000 \\
530 \\
414\end{array}$ \\
\hline 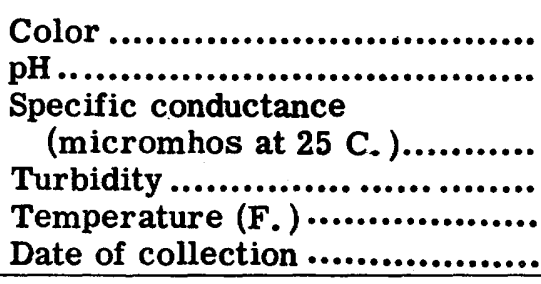 & $\begin{array}{r}3 \\
7.3 \\
430 \\
0 \\
75 \\
\text { Dec. } 4,1951 \\
\end{array}$ & $\begin{array}{r}2 \\
7.4 \\
436 \\
1 \\
76 \\
\text { Dec. } 4,1951 \\
\end{array}$ & $\begin{array}{r}7.3 \\
7 \\
1,450 \\
0 \\
80 \\
\text { Dec. } 4,1951 \\
\end{array}$ \\
\hline 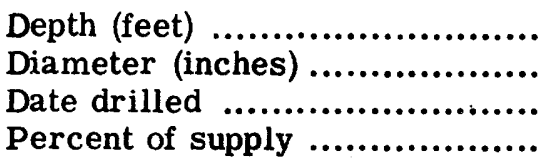 & $\begin{array}{r}1,000 \\
18-12 \\
1944 \\
--\end{array}$ & $\begin{array}{r}750 \\
8 \\
1945 \\
--\end{array}$ & \\
\hline
\end{tabular}




\section{CEDARTOWN \\ (Population, 9, 470)}

Ownership: Municipal; also supplies suburban districts. Total population supplied, about 10,250 .

Source: Spring.

Treatment: Chlorination.

Raw-water storage: 2 standpipes, 185,000 and 1,575,000 gal.

Finished-water storage: None.

\section{ANALYSIS}

(Analysis, in parts per million, by U. S. Geological Survey)

\begin{tabular}{|c|c|c|c|}
\hline & $\begin{array}{c}\text { Finished } \\
\text { water }\end{array}$ & & $\begin{array}{c}\text { Finished } \\
\text { water }\end{array}$ \\
\hline $\begin{array}{l}\text { Silica }\left(\mathrm{SiO}_{2}\right) \quad \ldots \ldots \ldots \ldots \ldots . . \\
\text { Iron }(\mathrm{Fe}) \quad \ldots \ldots \ldots \ldots \ldots \ldots . . \\
\text { Manganese }(\mathrm{Mn}) \ldots \ldots \ldots \ldots\end{array}$ & $\begin{array}{l}9.1 \\
.02 \\
.00\end{array}$ & $\begin{array}{l}\text { Hardness as } \mathrm{CaCO}_{\mathrm{s}}: \\
\text { Total .......................... } \\
\text { Noncarbonate .............. }\end{array}$ & $\begin{array}{r}139 \\
8 \\
\end{array}$ \\
\hline 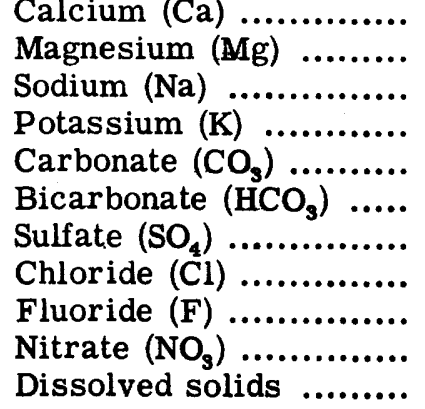 & $\begin{array}{c}31 \\
15 \\
.7 \\
0 \\
160 \\
3.5 \\
2.8 \\
.2 \\
1.7 \\
144\end{array}$ & 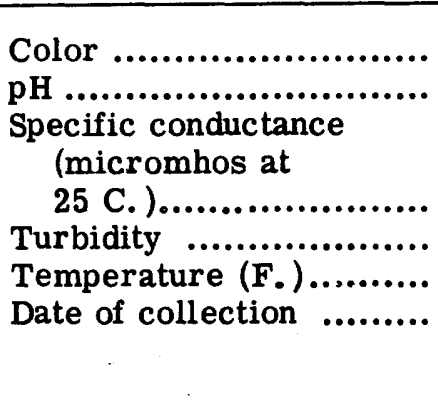 & $\begin{array}{r}253 \\
0 \\
62 \\
\text { Dec. } 29, \\
1951\end{array}$ \\
\hline
\end{tabular}

COLLEGE PARK

(Population, 14, 535)

Cwnership: Municipal.

Source: Supplied by East Point. (See East Point.) 


\section{COLUMBUS}

(Population, 79, 611)

Ownership: Municipal; also supplies Bealwood, Bibb City, and suburban districts. Total population supplied, about 120,000.

Source: Chattahoochee River.

Treatment: Coagulation with alum, sedimentation, rapid sand filtration, chlorination, and adjustment of $\mathrm{pH}$ with lime.

Rated capacity of treatment plant: $18,000,000 \mathrm{gpd}$.

Raw-water storage: Reservoir, 30,000,000 gal.

Finished-water storage: 3 clear wells, 3,000,000, 2, 500,000, and 250, $000 \mathrm{gal}$; 3 elevated tanks, 1,000,000 gal.

The treatment plant is about 4 miles north of the center of the city near the intake in the river. The water is pumped from the river into a raw-water reservoir from which it flows to the treatment plant.

\section{ANALYSES}

(Analyses, in parts per million, by U. S. Geological Survey)

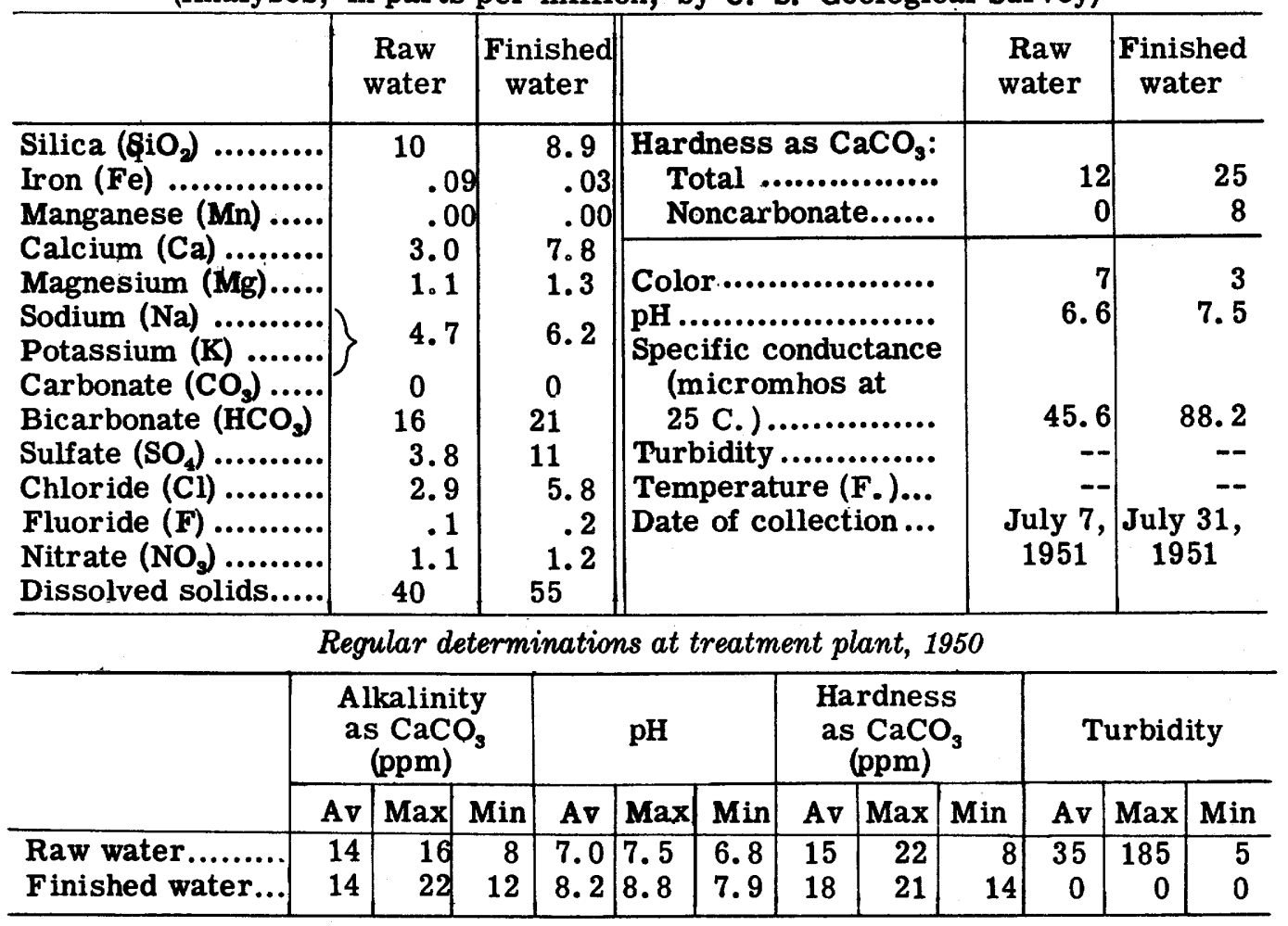




\section{CORDELE}

(Population, 9, 462)

Ownership: Municipal; also supplies suburban districts. Total population supplied, about 9,500 .

Source: 3 wells ( 1 to 3 ) 396,735 , and $540 \mathrm{ft}$ deep. The yield of well 1 is reported to be $900 \mathrm{gpm}$; of well 3, 1, $000 \mathrm{gpm}$; of well 2, not reported.

Treatment: Chlorination.

Raw-water storage: None.

Finished-water storage: Reservoir, 250,000 gal; standpipe, 250,000 gal.

\section{ANALYSES}

(Analyses, in parts per million, by U. S. Geological Survey)

\begin{tabular}{|c|c|c|c|c|}
\hline & Well 1 & Well 1 & $\begin{array}{c}\text { Well } 3 \\
\text { (raw water) }\end{array}$ & $\begin{array}{c}\text { Wells } 1,2,3 \\
\text { (finished } \\
\text { water) }\end{array}$ \\
\hline 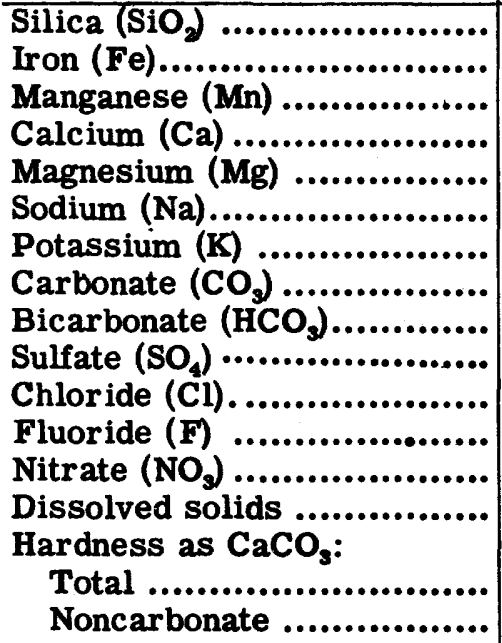 & $\begin{array}{r}13 \\
.01 \\
-- \\
44 \\
1.5 \\
3.4 \\
.5 \\
--- \\
135 \\
1.4 \\
4.8 \\
.0 \\
3.2 \\
142 \\
116 \\
5\end{array}$ & $134 \begin{array}{rr} & -- \\
0 & -- \\
& -- \\
- & -- \\
-- & -\end{array}$ & $\begin{array}{c}32 \\
.06 \\
.00 \\
52 \\
2.4 \\
2.5 \\
0 \\
163 \\
7.5 \\
2.4 \\
.1 \\
.0 \\
184 \\
140 \\
6\end{array}$ & $\begin{array}{c}31 \\
.09 \\
52.00 \\
2.4 \\
\\
0 \\
160 \\
7.0 \\
.9 \\
.0 \\
.0 \\
180 \\
140 \\
8\end{array}$ \\
\hline 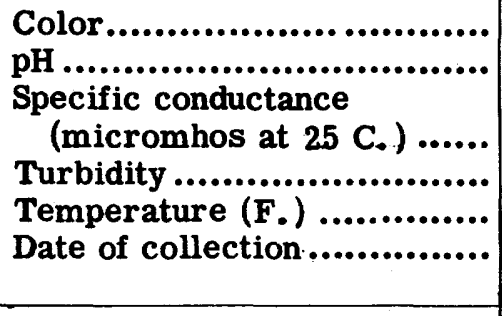 & $\begin{array}{r}-- \\
-- \\
-- \\
\text { Jan. } 21, \\
1938 \\
\end{array}$ & $\begin{array}{r}243 \\
-- \\
62 \\
1, \\
1951\end{array}$ & $\begin{array}{r}1 \\
7.5 \\
271 \\
0 \\
70 \\
\text { Dec. } 1 \\
1951 \\
\end{array}$ & $\begin{array}{r}3 \\
7.3 \\
269 \\
1 \\
63 \\
\text { Dec. } 1 \text {, } \\
1951 \\
\end{array}$ \\
\hline 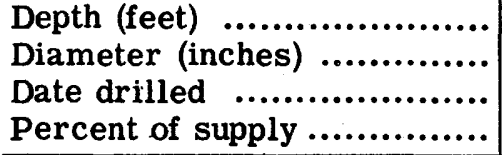 & $\begin{array}{r}396 \\
8 \\
1900 \\
--\end{array}$ & $\begin{array}{r}396 \\
8 \\
1900 \\
--\end{array}$ & $\begin{array}{r}540 \\
26-10 \\
1948 \\
-\end{array}$ & \\
\hline
\end{tabular}




\section{DALTON \\ (Population, 15, 968)}

Ownership: Municipal; also supplies suburban areas. Total population supplied, about 19,000 .

Source: Mill Creek. The intake is at the treatment plant about 1.4 miles northwest of the center of the city.

Treatment: Softening with lime, coagulation with ferric sulfate or alum, sedimentation, rapid sand filtration, and chlorination.

Rated capacity of treatment plant: 5,000,000 gpd.

Raw-water storage: None.

Finished-water storage: Clear well, 200,000 gal; 2 elevated tanks, 2, 000,000, and 750,000 gal.

\section{ANALYSES}

(Analyses, in parts per million, by U. S. Geological Survey)

\begin{tabular}{|c|c|c|c|c|c|}
\hline & $\begin{array}{c}\text { Raw } \\
\text { water }\end{array}$ & $\begin{array}{c}\text { Finished } \\
\text { water }\end{array}$ & & $\begin{array}{c}\text { Raw } \\
\text { water }\end{array}$ & $\begin{array}{l}\text { Finished } \\
\text { water }\end{array}$ \\
\hline \multirow{9}{*}{ 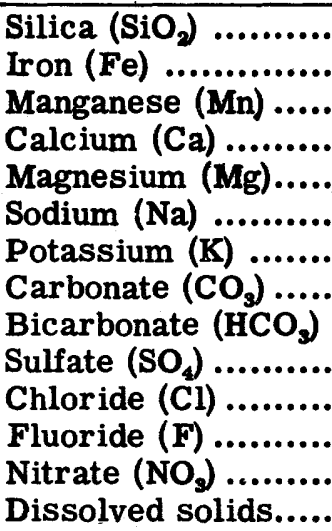 } & \multirow{2}{*}{$\begin{array}{c}9.2 \\
.03 \\
.00 \\
33 \\
8.6\end{array}$} & \multirow{2}{*}{$\begin{array}{c}8.5 \\
.01 \\
.00 \\
14 \\
8.2\end{array}$} & $\begin{array}{r}\text { Hardness as } \mathrm{CaCO}_{\mathbf{3}}: \\
\text { Total } . . . . . . . . . . . . . . . \\
\text { Noncarbonate...... }\end{array}$ & $\begin{array}{r}118 \\
3\end{array}$ & $\begin{array}{r}69 \\
6\end{array}$ \\
\hline & & & \multirow{8}{*}{$\begin{array}{l}\text { Color ..................... } \\
\text { pH ....................... } \\
\text { Specific conductance } \\
\text { (micromhos at } \\
25 \text { C.) ................. } \\
\text { Turbidity .............. } \\
\text { Temperature (F.)... } \\
\text { Date of collection... }\end{array}$} & \multirow{2}{*}{$\begin{array}{r}4 \\
7.3\end{array}$} & \multirow{2}{*}{$\begin{array}{r}3 \\
8.6\end{array}$} \\
\hline & 1.6 & 1.7 & & & \\
\hline & $\begin{array}{r}0 \\
140\end{array}$ & $\begin{array}{r}7 \\
62\end{array}$ & & 225 & 145 \\
\hline & 2.5 & 7.9 & & & \\
\hline & 2.0 & 2.4 & & 47 & \\
\hline & .1 & .1 & & Nov. 29, & Nov. 29, \\
\hline & .9 & .9 & & 1951 & 1951 \\
\hline & & & & & \\
\hline
\end{tabular}

Regular determinations at treatment plant, 1950

\begin{tabular}{|c|c|c|c|c|c|c|c|c|c|c|c|c|}
\hline & \multicolumn{3}{|c|}{$\begin{array}{l}\text { Alkalinity } \\
\text { as CaCO, } \\
\text { (ppm) }\end{array}$} & \multicolumn{3}{|c|}{$\mathrm{pH}$} & \multicolumn{3}{|c|}{$\begin{array}{c}\text { Hardness } \\
\text { as } \mathrm{CaCO}_{3} \\
\text { (ppm) }\end{array}$} & \multicolumn{3}{|c|}{ Turbidity } \\
\hline & Av & $\operatorname{Max}$ & Min & Av & Max & Min & Av & Max & Min & Av & $\operatorname{Max}$ & Min \\
\hline Raw water......... & $\begin{array}{r}100 \\
50\end{array}$ & $\begin{array}{r}125 \\
65\end{array}$ & $\begin{array}{l}10 \\
20 \\
\end{array}$ & 7.1 & $\begin{array}{l}7.3 \\
9.1\end{array}$ & 6.5 & $\begin{array}{r}100 \\
50\end{array}$ & $\begin{array}{r}140 \\
65\end{array}$ & 12 & 50 & 5000 & 10 \\
\hline
\end{tabular}


DECATUR

(Population, 21, 635)

Ownership: DeKalb County Water System; supplies Decatur and suburban areas, Avondale, Chamblee, Doraville, and a large number of consumers in DeKalb County. Total population supplied, about 93,000 .

Source: Chattahoochee River. The intake is at Holcombe Bridge about 22 miles north of Decatur.

Treatment: Coagulation with alum, sedimentation, rapid sand filtration, chlorination, fluoridation, and adjustment of $\mathrm{pH}$ with lime.

Rated capacity of treatment plant: $16,000,000 \mathrm{gpd}$.

Raw-water storage: Reservoir, 75,000,000 gal.

Finished-water storage: Clear well, 1,500,000 gal; 2 clear wells, 400,000 gal (owned by Decatur, leased by county); 3 elevated tanks, 1, 000,000, 500,000, and 85,000 gal.

\section{ANALYSES}

(Analyses, in parts per million, by U. S. Geological Survey)

\begin{tabular}{|c|c|c|c|c|c|}
\hline & $\begin{array}{l}\text { Raw } \\
\text { water }\end{array}$ & $\begin{array}{c}\text { Finisned } \\
\text { water }\end{array}$ & & $\begin{array}{c}\text { Raw } \\
\text { water }\end{array}$ & $\begin{array}{l}\text { Finished } \\
\text { water }\end{array}$ \\
\hline $\begin{array}{l}\text { Silica }\left(\mathrm{SiO}_{2}\right) \ldots \ldots \ldots \ldots . \\
\text { Iron }(\mathrm{Fe}) \ldots \ldots \ldots \ldots . . \\
\text { Manganese }(\mathrm{Mn}) \ldots \ldots\end{array}$ & $\begin{array}{r}12 \\
.06 \\
.00\end{array}$ & $\begin{array}{r}12 \\
.03 \\
.00\end{array}$ & $\begin{array}{r}\text { Hardness as } \mathrm{CaCO}_{3} \text { : } \\
\text { Total ................... } \\
\text { Noncarbonate...... }\end{array}$ & $\begin{array}{r}12 \\
0\end{array}$ & $\begin{array}{r}19 \\
5\end{array}$ \\
\hline $\begin{array}{l}\text { Calcium }(\mathrm{Ca}) \ldots . . . . . \\
\text { Magnesium }(\mathrm{Mg}) . . .\end{array}$ & $\begin{array}{l}3.0 \\
1.1\end{array}$ & $\begin{array}{l}5.8 \\
1.2\end{array}$ & Color ..................... & 9 & 2 \\
\hline $\begin{array}{l}\text { Sodium (Na) ........... } \\
\text { Potassium (K) }\end{array}$ & 4.1 & 3.4 & $\begin{array}{l}\text { pH ......................... } \\
\text { Specific conductance }\end{array}$ & 6.8 & 6.9 \\
\hline $\begin{array}{l}\text { Carbonate }\left(\mathrm{CO}_{3}\right) \ldots . . . \\
\text { Bicarbonate }\left(\mathrm{HCO}_{3}\right)\end{array}$ & $\begin{array}{r}0 \\
15\end{array}$ & $\begin{array}{r}0 \\
18\end{array}$ & $\begin{array}{l}\text { (micromhos at } \\
25 \text { C. )............... }\end{array}$ & 56.4 & 58.1 \\
\hline Sulfate $\left(\mathrm{SO}_{4}\right) \ldots \ldots \ldots$ & 3.6 & 5.6 & Turbidity ............... & 16 & 1 \\
\hline Chloride (Cl) .......... & 2.9 & 3.2 & Temperature (F.)... & 50 & 51 \\
\hline Fluoride (F) ........... & .2 & .6 & Date of collection... & Nov. 27, & Nov. 27, \\
\hline Nitrate $\left(\mathrm{NO}_{3}\right) \ldots \ldots \ldots$ & .2 & .2 & & 1951 & 1951 \\
\hline Dissolved solids..... & 42 & 40 & & & \\
\hline
\end{tabular}

Regular determinations at treatment plant, 1950

\begin{tabular}{l|r|r|r|r|r|r|r|r|r|r|r|r|r}
\hline & \multicolumn{3}{|c|}{$\begin{array}{c}\text { Alkalinity } \\
\text { as CaCO } \\
\text { (ppm) }\end{array}$} & \multicolumn{3}{c|}{ pH } & \multicolumn{3}{c|}{$\begin{array}{c}\text { Hardness } \\
\text { as CaCO } \\
\text { (ppm) }\end{array}$} & \multicolumn{3}{c}{ Turbidity } \\
\cline { 2 - 13 } & Av & Max & Min & Av & Max & Min & Av & Max & Min & Av & Max & Min \\
\hline Raw water......... & -- & -- & -- & -- & -- & -- & -- & -- & -- & -- & -- & -- \\
Finished water... & 20 & 23 & 19 & 8.0 & 8.5 & 7.8 & 30 & -- & -- & 38 & -- & -- \\
\hline
\end{tabular}




\section{DUBLIN}

(Population, 10, 232)

Ownership: Municipal; also supplies Veterans Hospital. Total population supplied, about 11,000 .

Source: 4 wells (1 to 4) $290,290,490$, and $650 \mathrm{ft}$ deep. The wells are all flowing wells. The yield of well 4 is reported to be $350 \mathrm{gpm}$.

Treatment: Aeration, ammoniation, softening with lime, chlorination, sedimentation, recarbonation, and rapid sand filtration.

Rated capacity of treatment plant: $1,500,000 \mathrm{gpd}$.

Raw-water storage: None.

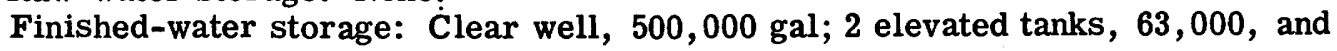
250,000 gal.

\section{ANALYSES}

(Analyses, in parts per million, by U. S. Geological Survey)

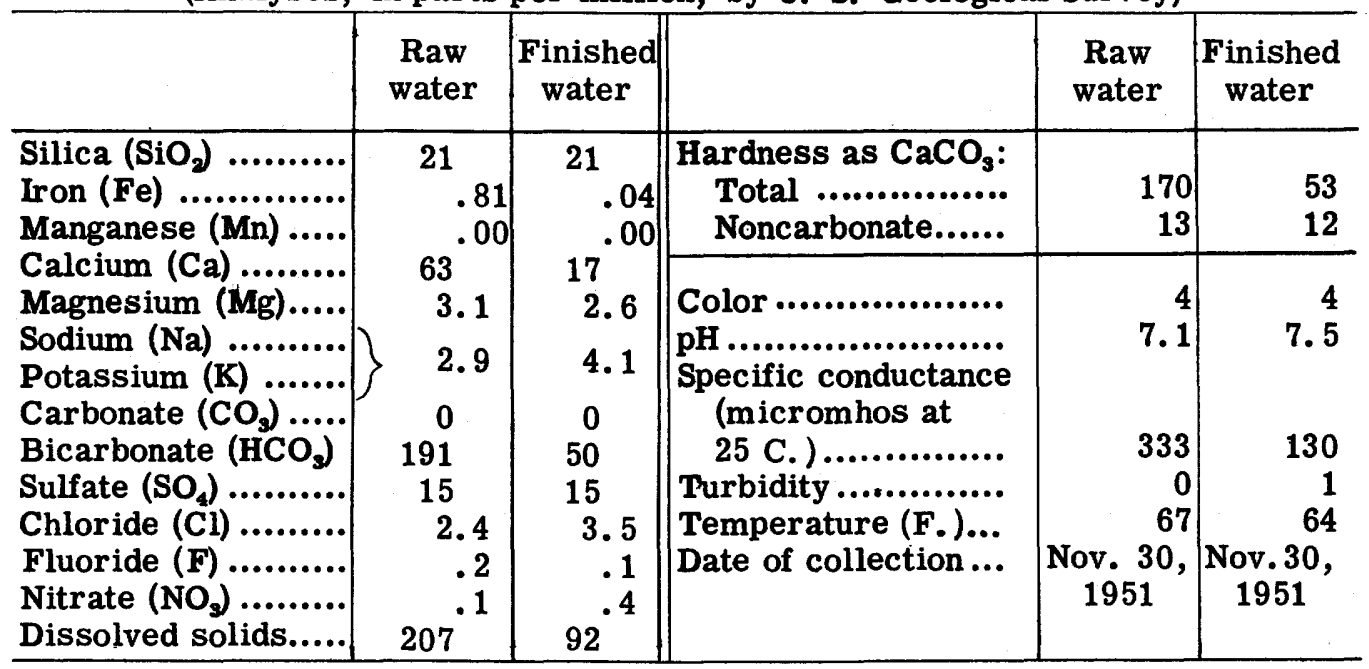

Regular determinations at treatment plant, 1950

\begin{tabular}{l|r|r|r|r|r|r|r|r|r|r|r|r}
\hline & \multicolumn{3}{|c|}{$\begin{array}{c}\text { Alkalinity } \\
\text { as CaCO } \\
\text { (ppm) }\end{array}$} & \multicolumn{3}{c|}{ pH } & \multicolumn{3}{c|}{$\begin{array}{c}\text { Hardness } \\
\text { as CaCO } \\
\text { (ppm) }\end{array}$} & \multicolumn{3}{c}{ Turbidity } \\
\cline { 2 - 25 } & Av & Max & Min & Av & Max & Min & Av & Max & Min & Av & Max & Min \\
\hline Raw water......... & 175 & 186 & 162 & 7.2 & 7.2 & 7.2 & 176 & 180 & 174 & -- & -- & -- \\
Finished water... & 54 & 77 & 40 & 8.5 & 8.6 & 8.4 & 66 & 88 & 58 & -- & -- & -- \\
\hline
\end{tabular}




\section{EAST POINT \\ (Population, 21, 080)}

Ownership: Municipal; also supplies College Park and suburban areas. Total population supplied, about 38,000 .

Source: Sweetwater Creek. The intake is about 12 miles west of East Point.

Treatment: Prechlorination, ammoniation, coagulation with alum, sedimentation, rapid sand filtration, chlorine dioxide, and adjustment of $\mathrm{pH}$ with lime.

Rated capacity of treatment plant: $4,000,000$ gpd.

Raw-water storage: Reservoir, 10,000,000 gal.(An additional reservoir of 47, 000, 000 gal capacity is being constructed).

Finished-water storage: Clear well, 750,000 gal; 2 elevated tanks, 500,000 gal each; 1 elevated tank (in College Park).

\section{ANALYSES}

(Analyses, in parts per million, by U. S. Geological Survey)

\begin{tabular}{|c|c|c|c|c|c|}
\hline & $\begin{array}{l}\text { Raw } \\
\text { water }\end{array}$ & $\begin{array}{c}\text { Finished } \\
\text { water }\end{array}$ & & $\begin{array}{c}\text { Raw } \\
\text { water }\end{array}$ & $\begin{array}{c}\text { Finished } \\
\text { water }\end{array}$ \\
\hline $\begin{array}{l}\text { Silica }\left(\mathrm{SiO}_{2}\right) \\
\text { Iron }(\mathrm{Fe}) \ldots \ldots \ldots \ldots \\
\text { Manganese }(\mathrm{Mn}) \ldots \ldots . . .\end{array}$ & $\begin{array}{l}19 \\
.03 \\
.00\end{array}$ & $\begin{array}{r}18 \\
.03 \\
.00\end{array}$ & $\begin{array}{r}\text { Hardness as } \mathrm{CaCO}_{3} \text { : } \\
\text { Total } \ldots . . . . . . . . . . . . . \\
\text { Noncarbonate..... }\end{array}$ & $\begin{array}{r}19 \\
0\end{array}$ & $\begin{array}{r}35 \\
7\end{array}$ \\
\hline $\begin{array}{l}\text { Calcium (Ca) .......... } \\
\text { Magnesium (Mg)..... }\end{array}$ & $\begin{array}{l}4.9 \\
1.7\end{array}$ & $\begin{array}{r}11 \\
1.9\end{array}$ & Color....................... & 12 & 3 \\
\hline $\begin{array}{l}\text { Sodium (Na) ............ } \\
\text { Potassium (K) ....... }\end{array}$ & 6.1 & 5.7 & $\begin{array}{l}\text { pH ........................ } \\
\text { Specific conductance }\end{array}$ & 7.3 & 8.6 \\
\hline $\begin{array}{l}\text { Carbonate }\left(\mathrm{CO}_{3}\right) \ldots . . . \\
\text { Bicarbonate }\left(\mathrm{HCO}_{3}\right)\end{array}$ & $\begin{array}{r}0 \\
24\end{array}$ & $\begin{array}{r}8 \\
18\end{array}$ & $\begin{array}{l}\text { (micromhos at } \\
25 \text { C. ) } \ldots . . . \ldots \ldots \ldots . . . .\end{array}$ & 79.1 & 96.6 \\
\hline $\begin{array}{l}\text { Sulfate }\left(\mathrm{SO}_{4}\right) \ldots \ldots \ldots \\
\text { Chloride }(\mathrm{Cl}) \ldots \ldots . . . .\end{array}$ & $\begin{array}{l}6.3 \\
3.9\end{array}$ & $\begin{array}{r}11 \\
5.0\end{array}$ & $\begin{array}{l}\text { Turbidity ............... } \\
\text { Temperature (F.)... }\end{array}$ & $\begin{array}{r}8 \\
50\end{array}$ & $\begin{array}{r}1 \\
51\end{array}$ \\
\hline $\begin{array}{l}\text { Fluoride }(\mathrm{F}) \ldots \ldots \ldots \ldots \\
\text { Nitrate }\left(\mathrm{NO}_{3}\right) \ldots \ldots \ldots \ldots\end{array}$ & $\begin{array}{r}.2 \\
.3\end{array}$ & $\begin{array}{l}.3 \\
.2\end{array}$ & Date of collection... & $\begin{array}{c}\text { Nov. 28, } \\
1951\end{array}$ & $\begin{array}{c}\text { Nov. 28, } \\
1951\end{array}$ \\
\hline & & 70 & & & \\
\hline
\end{tabular}

Regular determinations at treatment plant, 1950

\begin{tabular}{|c|c|c|c|c|c|c|c|c|c|c|c|c|}
\hline & \multicolumn{3}{|c|}{$\begin{array}{c}\text { Alkalinity } \\
\text { as } \mathrm{CaCO} \\
\text { (ppm) }\end{array}$} & \multicolumn{3}{|c|}{$\mathrm{pH}$} & \multicolumn{3}{|c|}{$\begin{array}{l}\text { Hardness } \\
\text { as } \mathrm{CaCO} \\
\text { (ppm) }\end{array}$} & \multicolumn{3}{|c|}{ Turbidity } \\
\hline & Av & $\operatorname{Max}$ & Min & Av & $\operatorname{Max}$ & Min & Av & $\operatorname{Max}$ & Min & Av & $\operatorname{Max}$ & Min \\
\hline $\begin{array}{l}\text { Raw water......... } \\
\text { Finished water... }\end{array}$ & $\begin{array}{l}17 \\
24\end{array}$ & \begin{tabular}{|l|}
28 \\
30
\end{tabular} & $\begin{array}{l}13 \\
20\end{array}$ & $\begin{array}{l}6.9 \\
8.7\end{array}$ & \begin{tabular}{|l|}
7.3 \\
9.2
\end{tabular} & $\begin{array}{l}6.7 \\
8.4\end{array}$ & $\begin{array}{l}16 \\
29\end{array}$ & $\begin{array}{l}20 \\
30\end{array}$ & $\begin{array}{l}14 \\
26\end{array}$ & 60 & $\begin{array}{r}320 \\
5\end{array}$ & $\begin{array}{r}10 \\
0\end{array}$ \\
\hline
\end{tabular}




\section{GAINESVILLE \\ (Population, 11, 936)}

Ownership: Municipal; also supplies suburban districts. Total population supplied, about 18,000 .

Source: Chattahoochee River (75 percent of supply); Crier Creek and Peeler Branch (25 percent of supply). The intake on Chattahoochee River is about 3.4 miles northeast of the center of the city.

Treatment: Prechlorination, coagulation with alum and lime, sedimentation, rapid sand filtration, and postchlorination when necessary.

Rated capacity of treatment plant: 2,000,000 gpd.

Raw-water storage: Impounding reservoir about 3,000,000 gal.

Finished-water storage: Clear well, 480,000 gal; 2 standpipes, 300,000 and 210,000 gal.

\section{ANALYSES}

(Analyses, in parts per million, by U. S. Geological Survey)

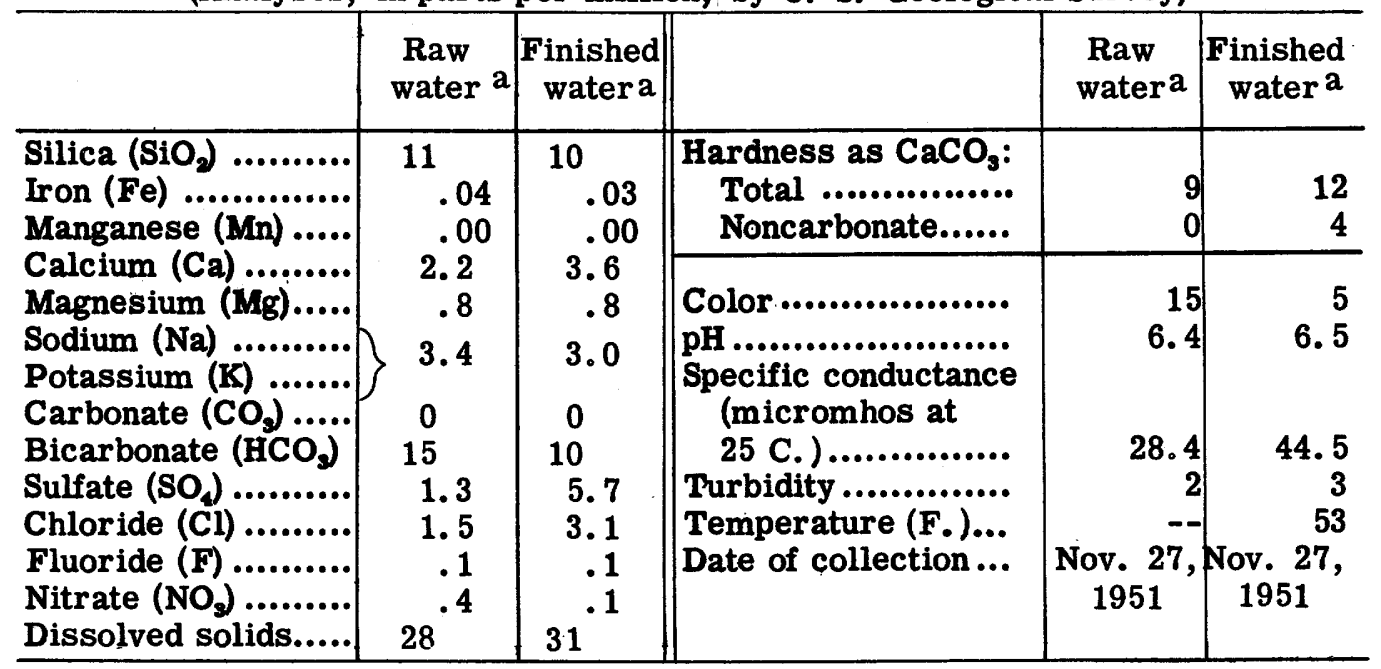

Regular determinations at treatment plant, 1950

\begin{tabular}{|c|c|c|c|c|c|c|c|c|c|c|c|c|}
\hline & \multicolumn{3}{|c|}{$\begin{array}{l}\text { Alkalinity } \\
\text { as CaCO } \\
\text { (ppm) }\end{array}$} & \multicolumn{3}{|c|}{ pH } & \multicolumn{3}{|c|}{$\begin{array}{c}\text { Hardness } \\
\text { as CaCO } \\
\text { (ppm) }\end{array}$} & \multicolumn{3}{|c|}{ Turbidity } \\
\hline & Av & Max & Min & Av & Max & Min & Av & Max & Min & Av & Max & Min \\
\hline $\begin{array}{l}\text { Raw water......... } \\
\text { Finished water... }\end{array}$ & $\begin{array}{l}10 \\
11\end{array}$ & $\begin{array}{l}12 \\
13\end{array}$ & $\begin{array}{l}8 \\
9\end{array}$ & $\begin{array}{l}6.9 \\
6.9\end{array}$ & $\begin{array}{l}7.2 \\
7.1\end{array}$ & \begin{tabular}{|l|}
6.8 \\
6.8
\end{tabular} & $\begin{array}{l}20 \\
25\end{array}$ & $\begin{array}{l}25 \\
28\end{array}$ & $\begin{array}{l}16 \\
18\end{array}$ & $\begin{array}{r}120 \\
1\end{array}$ & $\begin{array}{r}500 \\
1 \\
\end{array}$ & $\begin{array}{r}30 \\
1\end{array}$ \\
\hline
\end{tabular}

a Chattahoochee River 75 percent; Crier Creek and Peeler Branch 25 percent. 


\section{GRIFFIN}

(Population, 13, 982)

Ownership: Municipal; also supplies suburban districts. Total population supplied, about 20,000 .

Source: Flint River. The intake is about 10 miles northwest of the city.

Treatment: Prechlorination during fall and winter months, coagulation with alum, activated carbon, sedimentation, rapid sand filtration, chlorination, and adjustment of $\mathbf{p H}$ with lime.

Rated capacity of treatment plant: 4,000,000 gpd.

Raw-water storage: None.

Finished-water storage: 2 clear. wells, 1,500,000 and 3,000,000 gal; 2 elevated tanks, 275, 000 and 300, 000 gal.

\section{ANALYSES}

(Analyses, in parts per million, by U. S. Geological Survey)

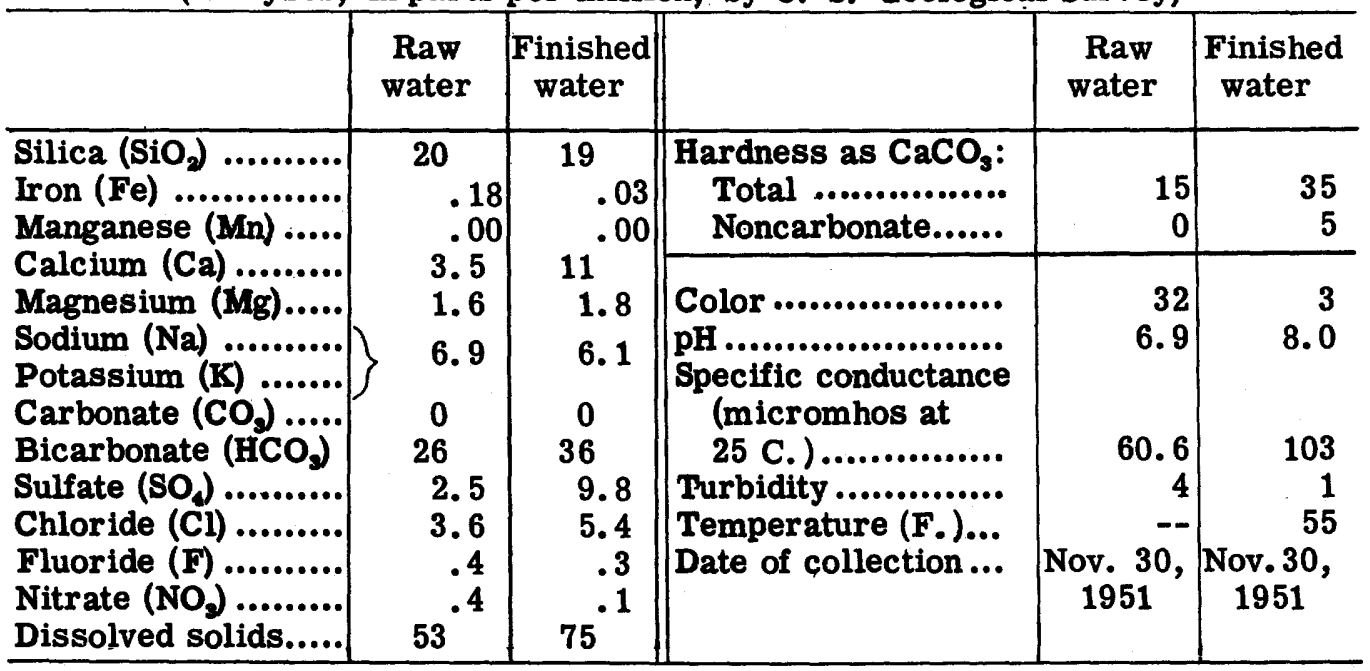

Regular determinations at treatment plant, 1950

\begin{tabular}{l|r|r|r|r|r|r|r|r|r|r|r|r}
\hline & \multicolumn{3}{|c|}{$\begin{array}{c}\text { Alkalinity } \\
\text { as CaCO } \\
\text { (ppm) }\end{array}$} & \multicolumn{3}{c|}{ pH } & \multicolumn{3}{c|}{$\begin{array}{c}\text { Hardness } \\
\text { as CaCO } \\
\text { (ppm) }\end{array}$} & \multicolumn{3}{c}{ Turbidity } \\
& \multicolumn{1}{|c|}{ Av } & Max & Min & Av & Max & Min & Av & Max & Min & Av & Max & Min \\
\hline Raw water......... & 20 & 28 & 13 & 6.8 & 7.0 & 6.5 & 18 & 21 & 16 & 35 & 700 & 20 \\
Finished water... & 27 & 36 & 19 & 8.0 & 8.4 & 7.8 & 28 & 32 & 26 & .15 & .20 & .10 \\
\hline
\end{tabular}


LAGRANGE

(Population, 25, 025)

Ownership: Municipal; also supplies suburban districts. Total population supplied, about 25, 500 .

Source: Chattahoochee River. The intake in the river is about 3.5 miles west of the city.

Treatment: Coagulation with alum and lime, chlorination, sedimentation, rapid sand filtration, and adjustment of $\mathrm{pH}$ with lime.

Rated capacity of treatment plant: 6,000,000 gal.

Raw-water storage: Reservoir, 12,000,000 gal.

Finished-water storage: Clear well, 1,000,000 gal; elevated tank, 1,000,000 gal; standpipe, 300,000 gal.

\section{ANALYSES}

(Analyses, in parts per million, by U. S. Geological Survey)

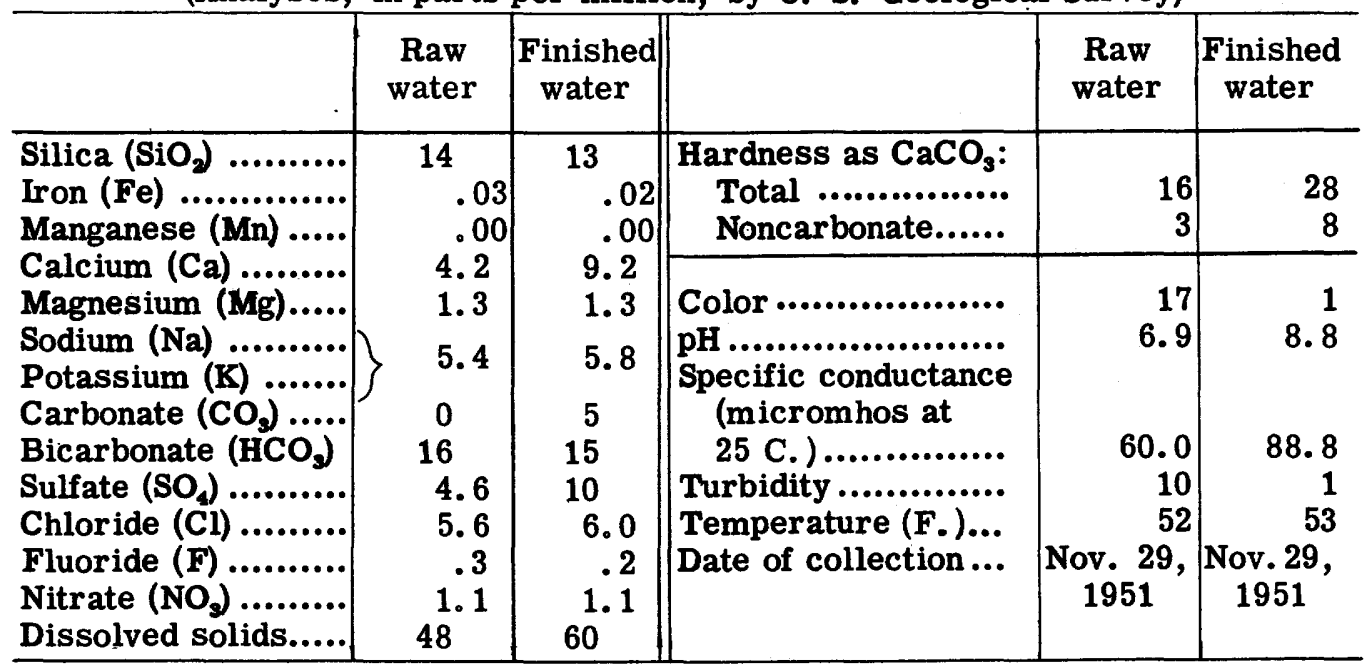

Regular determinations at treatment plant, 1950

\begin{tabular}{l|r|r|r|r|r|r|r|r|r|r|r|r}
\hline & \multicolumn{3}{|c|}{$\begin{array}{c}\text { Alkalinity } \\
\text { as CaCO } \\
\text { (ppm) }\end{array}$} & \multicolumn{3}{c|}{ pH } & \multicolumn{3}{c|}{$\begin{array}{c}\text { Hardness } \\
\text { as CaCO } \\
\text { (ppm) }\end{array}$} & \multicolumn{3}{c}{ Turbidity } \\
\cline { 2 - 13 } & Av & Max & Min & Av & Max & Min & Av & Max & Min & Av & Max & Min \\
\hline Raw water......... & 15 & 20 & 8 & 6.8 & 7.2 & 6.4 & 20 & -- & -- & -- & 300 & 10 \\
Finished water... & 28 & 30 & 26 & 8.7 & 9.0 & 8.4 & 28 & -- & -- & -- & 5 & .5 \\
\hline
\end{tabular}




\section{MACON}

(Population, 70, 252)

Ownership: Municipal; also supplies suburban districts. Total population supplied, about 100,000 .

Source: Ocmulgee River. The intake in the river is about 3 miles north of the center of the city.

Treatment: Coagulation with alum, ammoniation, chlorination, activated carbon at times, sedimentation, rapid sand filtration, postchlorination, and final adjustment of $\mathrm{pH}$ with lime.

Rated capacity of treatment plant: $12,000,000$ gal.

Raw-water storage: None.

Finished-water storage: Clear well, 275,000 gal; 2 reservoirs, 1, 500,000 and $3,000,000$ gal; 2 standpipes, 200,000 and 1,000,000 gal.

\section{ANALYSES}

(Analyses, in parts per million, by U. S. Geological Survey)

\begin{tabular}{|c|c|c|c|}
\hline & $\begin{array}{c}\text { Raw } \\
\text { water a }\end{array}$ & $\begin{array}{r}\text { Raw } \\
\text { water }\end{array}$ & $\begin{array}{l}\text { Finished } \\
\text { water }\end{array}$ \\
\hline 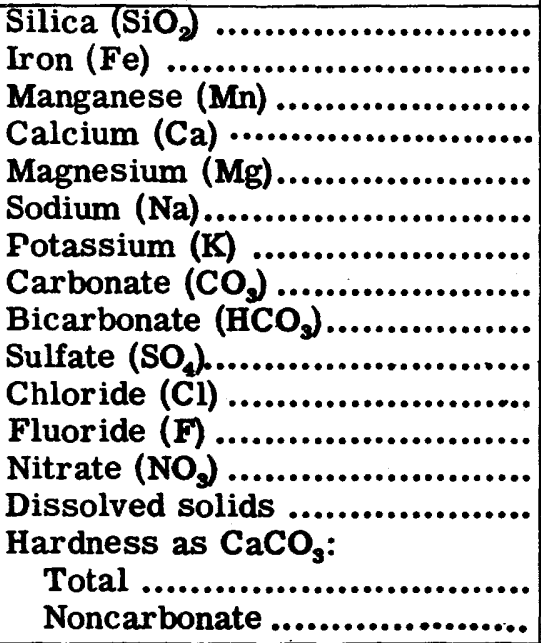 & $\begin{array}{r}12 \\
.06 \\
-- \\
3.6 \\
1.6 \\
4.6 \\
1.6 \\
-- \\
22 \\
3.9 \\
2.9 \\
.0 \\
1.0 \\
44 \\
16 \\
--\end{array}$ & $\begin{array}{c}5.6 \\
0 \\
21 \\
3.3 \\
3.6 \\
.2 \\
.7 \\
42 \\
15 \\
0\end{array}$ & $\begin{array}{l}12 \\
.03 \\
.00 \\
10 \\
1.6 \\
4.9 \\
0 \\
25 \\
14 \\
4.5 \\
.1 \\
.7 \\
64 \\
32 \\
11\end{array}$ \\
\hline 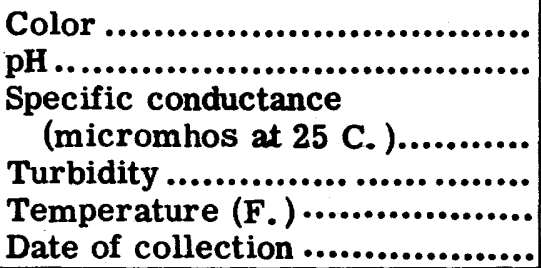 & $1937-38$ & $\begin{array}{r}7.0 \\
55.5 \\
-- \\
--1 \\
\text { Apr. 26, 1951 }\end{array}$ & $\begin{array}{r}3 \\
7.2 \\
98.2 \\
-- \\
-- \\
\text { Apr. } 26,1951\end{array}$ \\
\hline
\end{tabular}

Regular determinations at treatment plant, 1950

\begin{tabular}{l|r|r|r|r|r|r|r|r|r|r|r|r|r}
\hline & \multicolumn{3}{|c|}{$\begin{array}{c}\text { Alkalinity } \\
\text { as CaCO } \\
\text { (ppm) }\end{array}$} & \multicolumn{3}{c|}{ pH } & \multicolumn{3}{c|}{$\begin{array}{c}\text { Hardness } \\
\text { as CaCO } \\
\text { (ppm) }\end{array}$} & \multicolumn{3}{c}{ Turbidity } \\
\cline { 2 - 13 } & Av & Max & Min & Av & Max & Min & Av & Max & Min & Av & Max & Min \\
\hline Raw water......... & 15 & 16 & 15 & 7.4 & 7.2 & 7.2 & -- & -- & -- & -- & 35 & 18 \\
Finished water... & 25 & 26 & 24 & 8.5 & 8.6 & 7.4 & -- & -- & -- & 0 & 0 & 0 \\
\hline
\end{tabular}

a Average of 36 analyses of 10-day composites of daily samples, May 1, 1937, to Apr. 30, 1938. (U. S. Geol. Survey Water Supply Paper 889-E. 1944) 


\section{MARIETTA}

(Population, 20,687)

Ownership: Municipal; also supplies suburban districts. Total population supplied, about 20,700.

Source: Finished water from City of Atlanta, 2/3 of supply; 21 wells, $1 / 3$ of supply. The wells range in depth from 259 to $910 \mathrm{ft}$ and are reported to yield a total of $567 \mathrm{gpm}$.

Treatment: Well water: none.

Storage: 2 reservoirs, 243,000 and 1,000,000 gal; 2 standpipes, 500,000 gal each.

\section{ANALYSES}

(Analyses, in parts per million, by U. S. Geological Survey)

\begin{tabular}{|c|c|c|c|c|c|}
\hline & Well $8^{a}$ & Well $18^{a}$ & Well $21^{a}$ & Well $25^{a}$ & Wells b \\
\hline 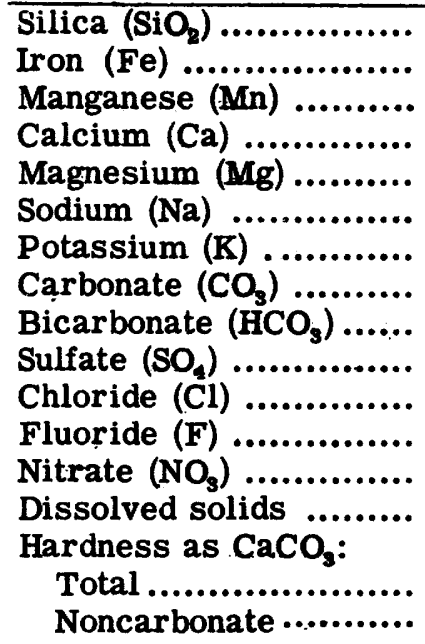 & $\begin{array}{l}19 \\
.10 \\
6.0 \\
3.6 \\
3.1 \\
1.5 \\
31-- \\
310 \\
4.0 \\
.0 \\
2.5 \\
59 \\
30 \\
4\end{array}$ & $\begin{array}{r}34 \\
.09 \\
32 \\
10 \\
15 \\
3.1 \\
{ }^{--} \\
103 \\
25 \\
16 \\
28 \\
226 \\
121 \\
37\end{array}$ & $\begin{array}{l}27 \\
.50 \\
10^{--} \\
3.3 \\
5.4 \\
2.2 \\
40^{--} \\
16 \\
1.8 \\
.0 \\
.2 \\
88^{.2} \\
39 \\
6\end{array}$ & $\begin{array}{l}22 \\
.43 \\
9.8 \\
3.9 \\
12 \\
1.8 \\
33 \\
3 .- \\
3.4 \\
15 \\
.0 \\
20 \\
119 \\
40 \\
13\end{array}$ & $\begin{array}{c}33 \\
.06 \\
.00 \\
16 \\
8.7 \\
4.5 \\
0 \\
73 \\
8.0 \\
7.4 \\
.0 \\
8.4 \\
123 \\
76 \\
16\end{array}$ \\
\hline $\begin{array}{l}\text { Color ........................ } \\
\text { pH ........................... } \\
\text { Specific conductance } \\
\quad \text { (micromhos at } 25 \text { C.) } \\
\text { Turbidity ................... } \\
\text { Temperature (F.) ......... } \\
\text { Date of collection ........ }\end{array}$ & 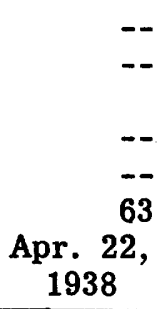 & $\begin{array}{c}-- \\
-- \\
-- \\
62 \\
\text { Apr. } 22, \\
1938\end{array}$ & $\begin{array}{r}-- \\
-- \\
-- \\
-- \\
62 \\
\text { Apr. } 22, \\
1938\end{array}$ & $\begin{array}{c}-- \\
-- \\
-- \\
-- \\
62 \\
\text { Apr. } 22, \\
1938\end{array}$ & $\begin{array}{r}2 \\
6.9 \\
180 \\
1 \\
62 \\
\text { Nov. } 28, \\
1951\end{array}$ \\
\hline $\begin{array}{l}\text { Depth (feet) } \ldots \ldots \ldots \ldots \ldots . . \\
\text { Diameter (inches)......... } \\
\text { Date drilled ............... } \\
\text { Percent of supply ........ }\end{array}$ & $\begin{array}{r}272 \\
10 \\
-- \\
--\end{array}$ & $\begin{array}{r}910 \\
10 \\
1927 \\
--\end{array}$ & $\begin{array}{r}382 \\
10 \\
1928 \\
--\end{array}$ & $\begin{array}{r}297 \\
10 \\
1937 \\
--\end{array}$ & \\
\hline
\end{tabular}

a U. S. Geol. Water Supply Paper 912. 1942

b Composite sample of water from wells $1,3,5$, and 8 . 
MOULTRIE

(Population, 11, 639)

Ownership: Municipal; also supplies suburban districts. Total population supplied, about 17,600 .

Source: 3 wells ( 1 to 3 ) 750,825 , and $752 \mathrm{ft}$ deep. The yield of wells 2 and 3 is reported to be 1,350 and $831 \mathrm{gpm}$ respectively.

Treatment: Chlorination.

Raw-water storage: None.

Finished-water storage: 2 reservoirs, 250,000 gal each; 2 elevated tanks, 150,000 and $500,000 \mathrm{gal}$.

\section{ANALYSES}

(Analyses, in parts per million, by U. S. Geological Survey)

\begin{tabular}{|c|c|c|c|c|c|}
\hline & $\begin{array}{l}\text { Well } 2 \\
\text { (raw } \\
\text { water) }\end{array}$ & $\begin{array}{c}\text { Well 3 } \\
\text { (finished } \\
\text { water) }\end{array}$ & & $\begin{array}{l}\text { Well } 2 \\
\text { (raw } \\
\text { water) }\end{array}$ & $\begin{array}{c}\text { Well 3 } \\
\text { (finished } \\
\text { water) }\end{array}$ \\
\hline \multirow{8}{*}{ 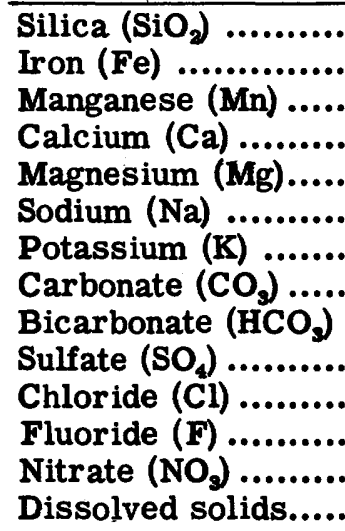 } & \multirow{2}{*}{$\begin{array}{c}26 \\
.05 \\
24 \\
13\end{array}$} & \multirow{2}{*}{\begin{tabular}{|l|}
${ }^{24}$ \\
.07 \\
$8^{86}$ \\
43
\end{tabular}} & $\begin{array}{c}\text { Hardness as } \mathrm{CaCO}_{3} \\
\text { Total } . . . . . . . . . . . . . . . \\
\text { Noncarbonate...... }\end{array}$ & $\begin{array}{r}113 \\
4\end{array}$ & $\begin{array}{l}391 \\
283\end{array}$ \\
\hline & & & \multirow{7}{*}{$\begin{array}{l}\text { Color ..................... } \\
\text { pH ......................... } \\
\text { Specific conductance } \\
\quad \text { (micromhos at } \\
25 \text { C. ) ................ } \\
\text { Turbidity .............. } \\
\text { Temperature (F.)... } \\
\text { Date of collection... }\end{array}$} & \multirow{2}{*}{$\begin{array}{r}2 \\
7.7\end{array}$} & 7 \\
\hline & 26 & 34 & & & \multirow{6}{*}{$\begin{array}{r}7.2 \\
859 \\
1 \\
70 \\
\text { Dec. } 2 \\
1951\end{array}$} \\
\hline & \multirow{5}{*}{$\begin{array}{r}0 \\
134 \\
48 \\
6.6 \\
.6 \\
210^{\circ}\end{array}$} & \multirow{5}{*}{$\begin{array}{r}0 \\
132 \\
329 \\
8.6 \\
.9 \\
635^{.4}\end{array}$} & & \multirow{5}{*}{$\begin{array}{r}333 \\
0 \\
74 \\
\text { Dec. 2, } \\
1951\end{array}$} & \\
\hline & & & & & \\
\hline & & & & & \\
\hline & & & & & \\
\hline & & & & & \\
\hline \multirow{4}{*}{\multicolumn{4}{|c|}{ 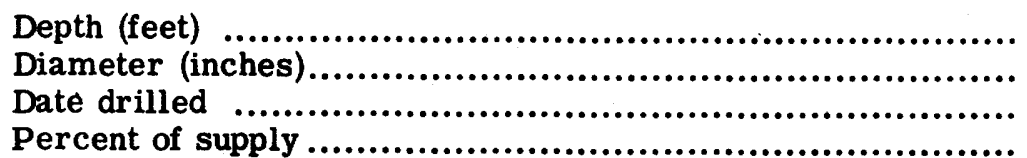 }} & 825 & 752 \\
\hline & & & & 12 & 16 \\
\hline & & & & 1943 & $1948-49$ \\
\hline & & & & & \\
\hline
\end{tabular}




\section{NEWNAN \\ (Population, 8, 218)}

Ownership: Municinal; also supplies suburban districts. Total population supplied, about 10,000 .

Source: 4 springs 80 percent of supply; 5 wells $(1,2,4$ to 6$) 400,806,350,350$, and $350 \mathrm{ft}$ deep, 20 percent of supply.

Treatment: Prechlorination, coagulation with alum and lime, sedimentation, rapid sand filtration, postchlorination, and aeration at times.

Rated capacity of treatment plant: $3,000,000 \mathrm{gpd}$.

Raw-water storage: Reservoir, 130,000,000 gal.

Finished-water storage: 2 clear wells, 250,000 and 1,250,000 gal; elevated tank, 500,000 gal; standpipe, 300,000 gal.

\section{ANALYSES}

(Analyșes, in parts per million, by U. S. Geological Survey)

\begin{tabular}{|c|c|c|c|}
\hline & $\begin{array}{c}\text { Raw } \\
\text { water a }\end{array}$ & $\begin{array}{c}\text { Raw } \\
\text { water b }\end{array}$ & $\begin{array}{c}\text { Finished } \\
\text { water c }\end{array}$ \\
\hline 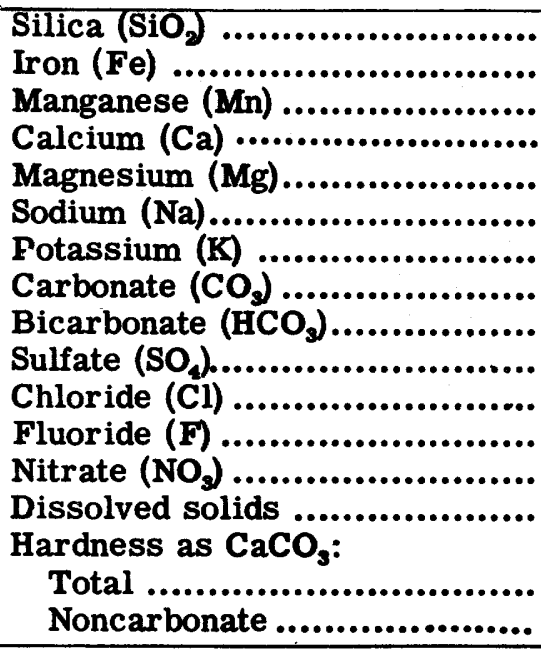 & $\begin{array}{r}0 \\
21 \\
2.5 \\
3.5 \\
.2 \\
.9 \\
41 \\
\\
17 \\
0 \\
\end{array}$ & $\begin{array}{r}0 \\
43 \\
27 \\
2.4 \\
.2 \\
.6 \\
105 \\
\\
48 \\
13\end{array}$ & $\begin{array}{c}15 \\
12 \\
14 \\
6.8 \\
.4 \\
.2 \\
82 \\
\\
48 \\
13 \\
\end{array}$ \\
\hline 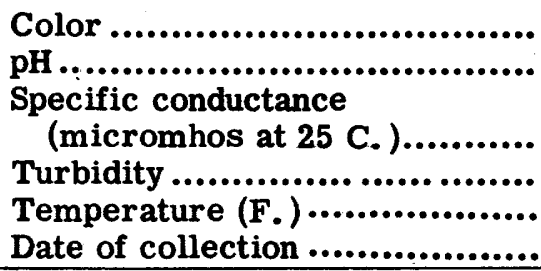 & $\begin{array}{r}5 \\
6.6 \\
53.4 \\
1 \\
54 \\
\text { Nov. } 29,1951 \\
\end{array}$ & $\begin{array}{r}7.0 \\
144 \\
1 \\
-- \\
\text { Nov. } 29,1951\end{array}$ & $\begin{array}{r}3 \\
9.4 \\
123 \\
1 \\
55 \\
\text { Nov. } 29,1951 \\
\end{array}$ \\
\hline
\end{tabular}

Regular determinations at treatment plant, 1950

\begin{tabular}{l|r|r|r|r|r|r|r|r|r|r|r|r|r}
\hline & \multicolumn{3}{|c|}{$\begin{array}{c}\text { Alkalinity } \\
\text { as CaCO } \\
\text { (ppm) }\end{array}$} & \multicolumn{3}{c|}{ pH } & \multicolumn{3}{c|}{$\begin{array}{c}\text { Hardness } \\
\text { as CaCO } \\
\text { (ppm) }\end{array}$} & \multicolumn{3}{c}{ Turbidity } \\
\cline { 2 - 13 } & Av & Max & Min & Av & Max & Min & Av & Max & Min & Av & Max & Min \\
\hline Raw water......... & 15 & 18 & 13 & 7.0 & 7.2 & 6.8 & -- & -- & -- & 40 & 60 & 20 \\
Finished water... & 22 & 28 & 16 & 8.4 & 8.8 & 8.0 & 36 & 46 & 26 & .05 & 1 & 0 \\
\hline
\end{tabular}

a From 4 springs.

b Mixed sample from wells 4, 5, and 6 .

c From 4 springs (80 percent), and wells (20 percent). 
ROME

(Population, 29, 615)

Ownership: Municipal; also supplies suburban districts. Total population supplied, about 35,000.

Source: Oostanaula River. The intake is 2 miles north of the center of the city.

Treatment: Coagulation with alum, chlorination, ammoniation, carbon, sedimentation, rapid sand filtration, and adjustment of $\mathrm{pH}$ with lime.

Rated capacity of treatment plant: 6,000,000 gpd.

Raw-water storage: None.

Finished-water storage: Clear well, 1,000,000 gal; reservoir, 3,000,000 gal.

\section{ANALYSES}

(Analyses, in parts per million, by U. S. Geological Survey)

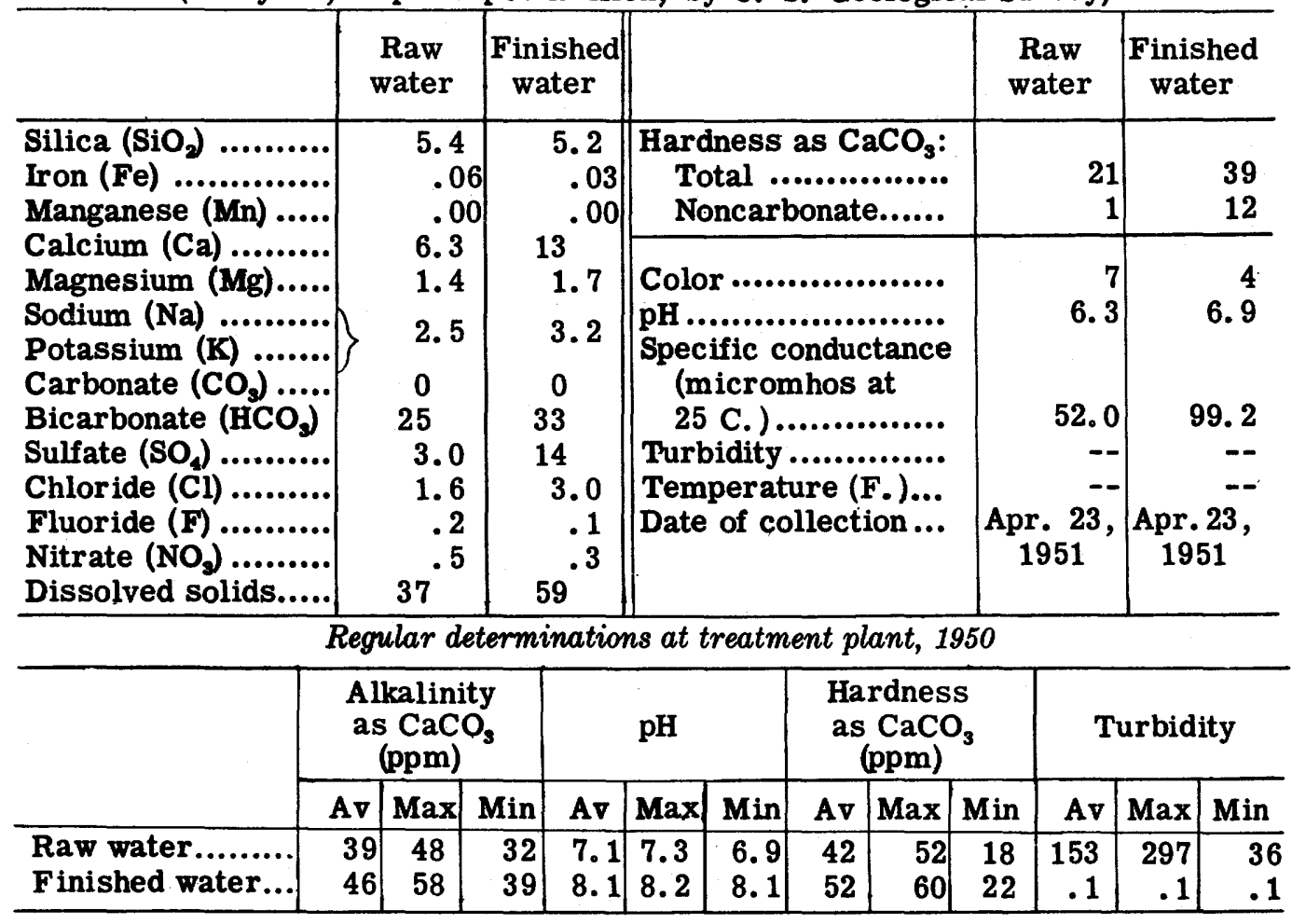




\section{SAVANNAH}

(Population, 119, 638)

Ownership: Municipal; also supplies suburban districts. Total population supplied, about 120,000 .

Source: 5 Artesian wells (5, 7 to 10) 60 percent of supply; Abercorn Creek 40 percent of supply. The intake in the creek is about 15 miles north of the city. The wells are $82,56,68,59$ and $62 \mathrm{ft}$ deep; the yield is reported to be 3,470 , $2,800,3,200,3,470$, and $1,400 \mathrm{gpm}$, respectively.

Treatment: Coagulation with alum, lime, and clay, sedimentation, rapid sand filtration, chlorination, and adjustment of $\mathrm{pH}$ with lime.

Rated capacity of treatment plant: $40,000,000$ gpd.

Raw-water storage: None.

Finished-water storage: 2 clear wells, 500,000 gal each; elevated tank, 4,000, $0000 \mathrm{gal}$; reservoir, 2,000,000 gạ.

\section{ANALYSES}

(Analyses, in parts per million, by U. S. Geological Survey)

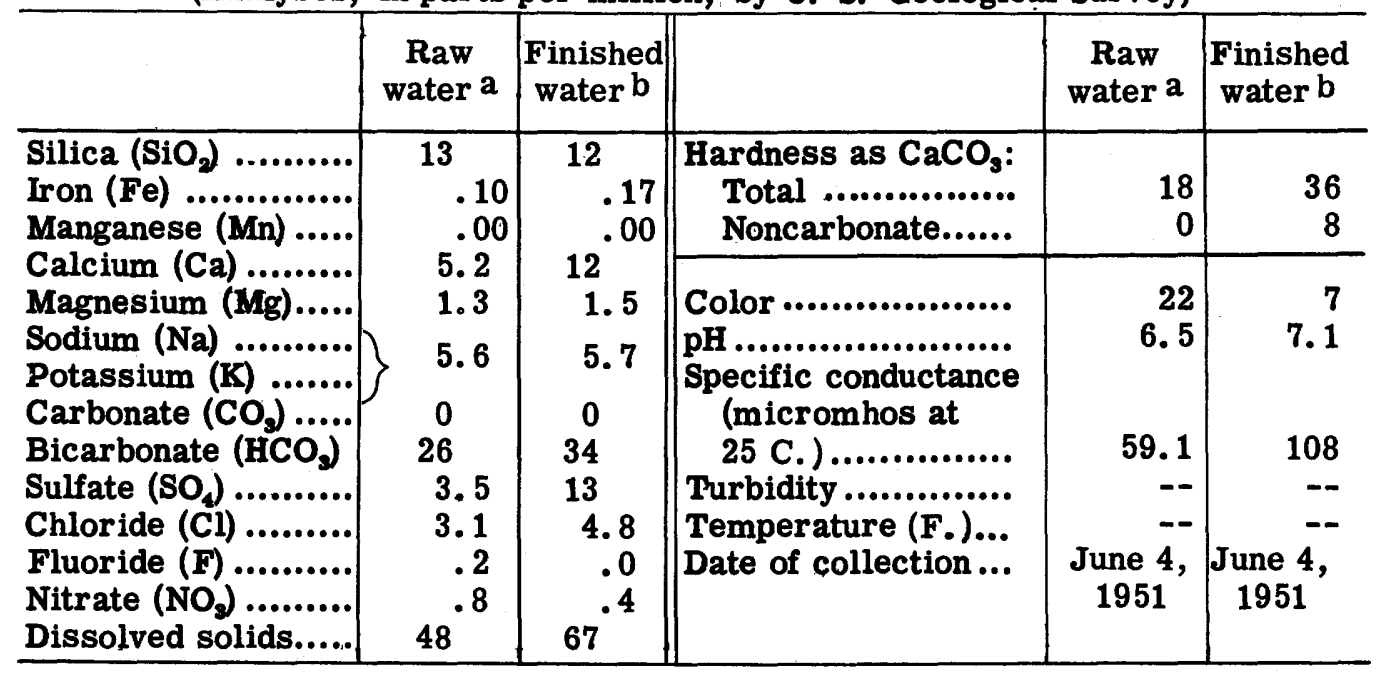

Regular determinations at treatment plant, 1950

\begin{tabular}{|c|c|c|c|c|c|c|c|c|c|c|c|c|}
\hline & \multicolumn{3}{|c|}{$\begin{array}{l}\text { Alkalinity } \\
\text { as CaCO } \\
\text { (ppm) }\end{array}$} & \multicolumn{3}{|c|}{ pH } & \multicolumn{3}{|c|}{$\begin{array}{c}\text { Hardness } \\
\text { as } \mathrm{CaCO} \\
(\mathrm{ppm})\end{array}$} & \multicolumn{3}{|c|}{ Turbidity } \\
\hline & Av & $\operatorname{Max}$ & Min & Av & $\operatorname{Max}$ & Min & Av & $\operatorname{Max}$ & Min & $A v$ & $\operatorname{Max}$ & Min \\
\hline $\begin{array}{l}\text { Raw water......... } \\
\text { Finished water... }\end{array}$ & \begin{tabular}{l|}
18 \\
23
\end{tabular} & $\begin{array}{l}26 \\
32\end{array}$ & $\begin{array}{l}10 \\
15\end{array}$ & $\begin{array}{l}6.6 \\
7.9\end{array}$ & $\begin{array}{l}7.2 \\
8.9\end{array}$ & $\begin{array}{l}6.0 \\
6.9\end{array}$ & $\begin{array}{l}19 \\
37\end{array}$ & $\begin{array}{l}26 \\
53\end{array}$ & \begin{tabular}{|l|}
12 \\
21 \\
\end{tabular} & $\begin{array}{r}70 \\
2.5\end{array}$ & $\begin{array}{r}125 \\
5\end{array}$ & $\begin{array}{r}15 \\
0\end{array}$ \\
\hline
\end{tabular}

a From Abercorn Creek.

b From wells and Abercorn Creek. 


\section{THOMASVILLE}

(Population, 14, 424)

Ownership: Municipal; also supplies suburban districts. Total population supplied, about 14,800 .

Source: 4 wells ( 2 to 5) $300,550,305$, and $400 \mathrm{ft}$ deep; yield reported to be 600 , $1,200,1,000$, and $1,300 \mathrm{gpm}$ respectively.

Treatment: Aeration, prechlorination, softening with lime and soda ash, coagulation with alum, bleaching clay, sedimentation, recarbonation, and rapid sand filtration.

Rated capacity of treatment plant: $1,500,000$ gpd.

Raw-water storage: None.

Finished-water storage: Reservoir, 500,000 gal; elevated tank, 300,000 gal.

\section{ANALYSES}

(Analyses, in parts per million, by U. S. Geological Survey)

\begin{tabular}{|c|c|c|c|c|c|}
\hline & $\begin{array}{l}\text { Well } 5 \\
\text { (raw } \\
\text { water) }\end{array}$ & $\begin{array}{c}\text { Finished } \\
\text { water }^{\text {a }}\end{array}$ & & $\begin{array}{l}\text { Well 5 } \\
\text { (raw } \\
\text { water) }\end{array}$ & $\begin{array}{l}\text { Finished } \\
\text { water a }\end{array}$ \\
\hline \multirow{7}{*}{ 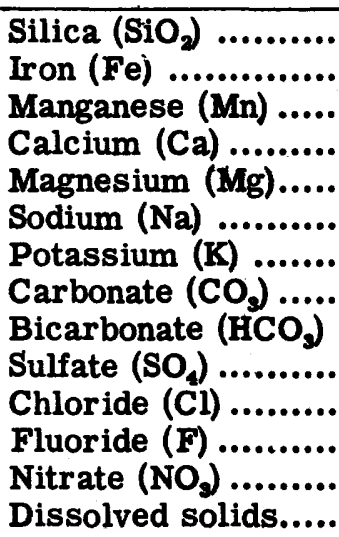 } & \multirow{2}{*}{$\begin{array}{l}24 \\
.07 \\
47 \\
.00 \\
22\end{array}$} & \multirow{2}{*}{\begin{tabular}{r|}
16 \\
.02 \\
23 \\
8.00 \\
8.9
\end{tabular}} & $\begin{array}{r}\text { Hardness as } \mathrm{CaCO}_{3} \text { : } \\
\text { Total ................. } \\
\text { Noncarbonate...... }\end{array}$ & $\begin{array}{r}208 \\
79\end{array}$ & $\begin{array}{l}94 \\
49\end{array}$ \\
\hline & & & \multirow{6}{*}{$\begin{array}{l}\text { Color .................... } \\
\text { pH ....................... } \\
\text { Specific conductance } \\
\text { (micromhos at } \\
25 \text { C.) ............... } \\
\text { Turbidity ............. } \\
\text { Temperature (F.)... } \\
\text { Date of collection... }\end{array}$} & & \\
\hline & 6.9 & 20 & & 7.9 & 9.2 \\
\hline & $\begin{array}{r}0 \\
157\end{array}$ & & & 416 & 289 \\
\hline & $\begin{array}{r}79 \\
7.6\end{array}$ & $\begin{array}{l}77 \\
8.8\end{array}$ & & & \\
\hline & $\begin{array}{r}.6 \\
.4\end{array}$ & $\begin{array}{r}8.8 \\
.4\end{array}$ & & Dec. 2, & Dec. 2, \\
\hline & $271^{.1}$ & $182^{.1}$ & & & \\
\hline \multirow{4}{*}{\multicolumn{4}{|c|}{ 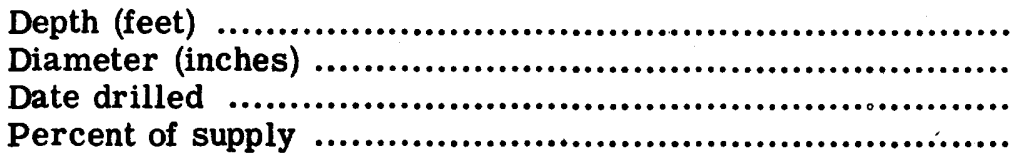 }} & 400 & \\
\hline & & & & & \\
\hline & & & & & \\
\hline & & & & & \\
\hline
\end{tabular}

Regular determinations at treatment plant, 1950

\begin{tabular}{|c|c|c|c|c|c|c|c|c|c|c|c|c|}
\hline & \multicolumn{3}{|c|}{$\begin{array}{c}\text { Alkalinity } \\
\text { as CaCO } \\
\text { (ppm) }\end{array}$} & \multicolumn{3}{|c|}{$\mathrm{pH}$} & \multicolumn{3}{|c|}{$\begin{array}{l}\text { Hardness } \\
\text { as } \mathrm{CaCO}_{3} \\
\text { (ppm) }\end{array}$} & \multicolumn{3}{|c|}{ Turbidity } \\
\hline & Av & $\operatorname{Max}$ & Min & $\mathbf{A v}$ & $\operatorname{Max}$ & $\operatorname{Min}$ & Av & $\operatorname{Max}$ & Min & $A \nabla$ & $\operatorname{Max}$ & Min \\
\hline $\begin{array}{l}\text { Raw water.......... } \\
\text { Finished water... }\end{array}$ & $\begin{array}{r}130 \\
32\end{array}$ & $\begin{array}{r}130 \\
47 \\
\end{array}$ & $\begin{array}{r}130 \\
27\end{array}$ & $\begin{array}{l}7.5 \\
8.9\end{array}$ & \begin{tabular}{|l|}
7.5 \\
9.6
\end{tabular} & $\begin{array}{l}7.5 \\
8.4\end{array}$ & $\begin{array}{r}200 \\
85\end{array}$ & \begin{tabular}{|l|}
200 \\
110 \\
\end{tabular} & $\begin{array}{r}200 \\
75\end{array}$ & $\begin{array}{l}0 \\
0\end{array}$ & \begin{tabular}{|l|}
0 \\
0
\end{tabular} & $\begin{array}{l}0 \\
0\end{array}$ \\
\hline
\end{tabular}

a From wells 3 and 4. 
VALDOSTA

(Population, 20, 046)

Ownership: Municipal; also supplies suburban districts. 'Total population supplied, about 23,000 .

Source: 3 wells (3, 4, Pendleton Park). The depths of wells 3 and 4 (Plant well) are $409 \mathrm{ft}$ and $380 \mathrm{ft}$, respectively.

Treatment: Aeration, chlorination, Nalco, lime.

Rated capacity of treatment plant: None.

Raw-water storage: None.

Finished-water storage: 2 reservoirs, 250,000 and 1,000,000 gal; 3 elevated tanks, 100,000, 400,000. and 500,000 gal.

\section{ANALYSES}

(Analyses, in parts per million, by U. S. Geological Survey)

\begin{tabular}{|c|c|c|c|c|}
\hline & Wel & & $\begin{array}{c}\text { Well 4 } \\
\text { (finished } \\
\text { water) }\end{array}$ & $\begin{array}{c}\text { Finished } \\
\text { water a }\end{array}$ \\
\hline 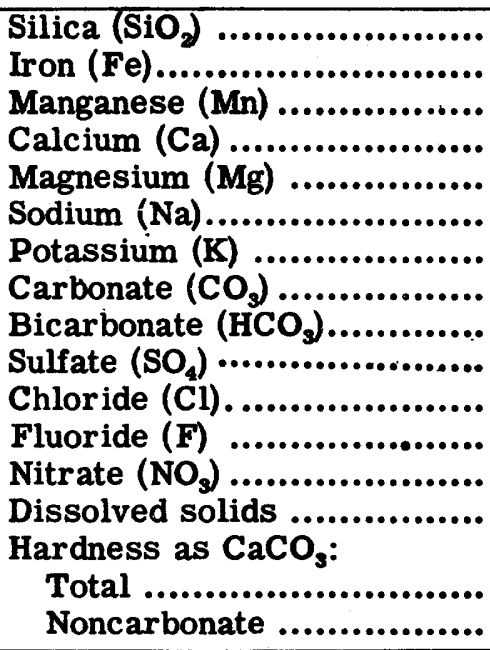 & $\begin{array}{c}15 \\
.01 \\
32 \\
4.4 \\
2.3 \\
1.0 \\
78 \\
78 \\
30 \\
3.8 \\
.2 \\
.3 \\
136 \\
98 \\
34\end{array}$ & \begin{tabular}{rr|}
23 & \\
& -- \\
-- & - \\
& - \\
0 & -- \\
80 & \\
26 & \\
& -- \\
& -- \\
& -- \\
& -- \\
101 &
\end{tabular} & $\begin{array}{l}16 \\
.12 \\
.00 \\
35 \\
5.0 \\
1.6 \\
0 \\
78 \\
27 \\
13 \\
.3 \\
.3 \\
143 \\
108 \\
44\end{array}$ & $\begin{array}{c}28 \\
.13 \\
.00 \\
24 \\
8.4 \\
.3 \\
0 \\
107 \\
4.1 \\
1.2 \\
.5 \\
.1 \\
128 \\
94 \\
7\end{array}$ \\
\hline 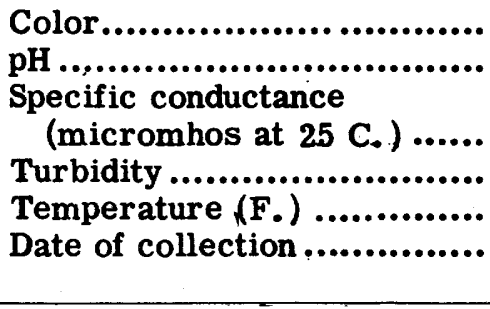 & $\begin{array}{c}-- \\
\\
\text { Sept. } 30 \text {, } \\
1941 \\
\end{array}$ & $\begin{array}{r}7.4 \\
218 \\
-- \\
68 \\
\text { Dec. } 2, \\
1951\end{array}$ & $\begin{array}{r}20 \\
7.3 \\
220 \\
1 \\
68 \\
\text { Dec. } 2 \text {, } \\
1951 \\
\end{array}$ & $\begin{array}{r}5 \\
7.9 \\
187 \\
1 \\
68 \\
\text { Dec. } 2 \text {, } \\
1951 \\
\end{array}$ \\
\hline 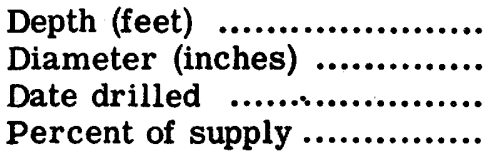 & $\begin{array}{r}409 \\
15 \\
1923 \\
--\end{array}$ & $\begin{array}{r}409 \\
15 \\
1923 \\
--\end{array}$ & $\begin{array}{r}380 \\
15 \\
1946 \\
--\end{array}$ & $19 \overline{--}$ \\
\hline
\end{tabular}

a Pendleton Park Well. 
WAYCROSS

(Population, 18, 899)

Ownership: Municipal; also supplies suburban districts.

Source: 2 wells (1 and 2) in Legion Park and at pumping plant 700 and $703 \mathrm{ft}$ deep.

The yield of wells is reported to be 1,700 and 2,000 gpm.

Treatment: Chlorination.

Raw-water storage: Reservoir, 798,830 gal.

Finished-water storage: Standpipe, 281,000 gal.

\section{ANALYSES}

(Analyses, in parts per million, by U. S. Geological Survey)

\begin{tabular}{|c|c|c|c|c|c|}
\hline & Well 1 & \begin{tabular}{|c|} 
Well 2 \\
(finished \\
water) \\
\end{tabular} & & Well 1 & \begin{tabular}{|c|}
$\begin{array}{c}\text { Well 2 } \\
\text { (finished } \\
\text { water) }\end{array}$ \\
\end{tabular} \\
\hline $\begin{array}{l}\text { Silica }\left(\mathrm{SiO}_{2}\right) \\
\text { Iron }(\mathrm{Fe}) \ldots \ldots \ldots \ldots . . . . . . . . \\
\text { Manganese }(\mathrm{Mn}) \ldots \ldots\end{array}$ & $\begin{array}{r}46 \\
.21 \\
--\end{array}$ & \begin{tabular}{r|}
43 \\
.11 \\
.00 \\
\end{tabular} & 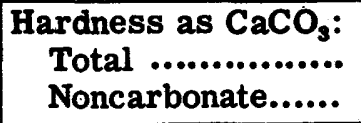 & $\begin{array}{r}142 \\
--\end{array}$ & $\begin{array}{r}172 \\
42\end{array}$ \\
\hline 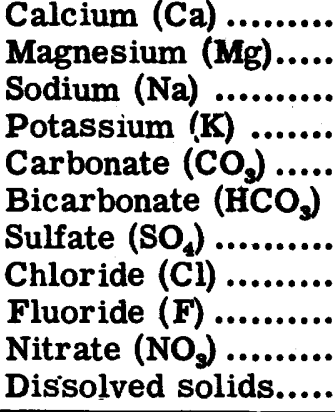 & $\begin{array}{r}34 \\
14 \\
16 \\
2.4 \\
159 \\
29 \\
14 \\
.4 \\
.224\end{array}$ & $\begin{array}{r}0 \\
159 \\
48 \\
18 \\
.5 \\
.1 \\
258\end{array}$ & $\begin{array}{l}\text { Color ................... } \\
\text { pH ......................... } \\
\text { Specific conductance } \\
\text { (micromhos at } \\
25 \text { C. ) ................ } \\
\text { Turbidity .............. } \\
\text { Temperature (F.)... } \\
\text { Date of collection... }\end{array}$ & $\begin{array}{r}74 \\
1941\end{array}$ & $\begin{array}{r}394 \\
0 \\
76 \\
\text { Dec } 3 \\
1951\end{array}$ \\
\hline \multicolumn{4}{|c|}{ 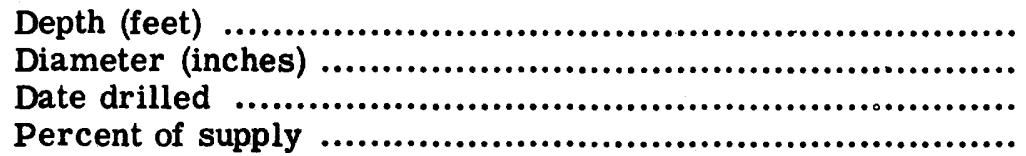 } & $\begin{array}{r}658 \\
12 \\
1893 \\
--\end{array}$ & $\begin{array}{r}703 \\
12-10 \\
1904 \\
--\end{array}$ \\
\hline
\end{tabular}




\section{ANNAPOLIS \\ (Population, 10, 047)}

Ownership: Municipal; supplies Dreams Landing, Eastport, Forest Hills, Germantown, Homewood, Wardour and West Annapolis. Total population supplied, about 28,000 .

Source: Broad Creek and other small streams (impounded), 74 percent of supply; 4 wells $(1,2,5$, and 6) 26 percent of supply. The wells are 270 , 250,248 , and $242 \mathrm{ft}$ deep respectively. Well number 1 is used very little. The surface water is used regularly, and the wells are used only to supplement this supply when necessary.

Treatment: Lime, aeration, coagulation with alum, sedimentation, rapid sand filtration, chlorination, and the addition of lime to adjust the $\mathrm{pH}$ to about 8.6. When the well water is used it is mixed with the surface water before treatment is begun.

Ratedi capacity of treatment plant: 5, 000, 000 gpd.

Raw-water storage: $80,000,000$ gal.

Finished-water storage: Basin, 500, 000 gal; standpipe, 1, 750, 000 gal.

The wells are used during the summer and during the peak demand furnish about one half of the supply. Most of this is furnished by wells 5 and 6 . During the year 1950 only well 5 was used. It is not possible at the present time to sample the individual well supplies.

\section{ANALYSES}

(Analyses, in parts per million, by U. S. Geological Survey)

\begin{tabular}{|c|c|c|c|c|c|}
\hline & $\begin{array}{c}\text { Raw } \\
\text { watera }\end{array}$ & $\begin{array}{l}\text { Finished } \\
\text { water }^{\mathrm{b}}\end{array}$ & & $\begin{array}{c}\text { Raw } \\
\text { watera }\end{array}$ & $\begin{array}{l}\text { Finished } \\
\text { water b }\end{array}$ \\
\hline 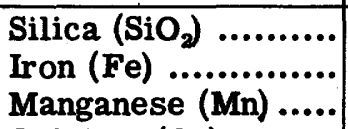 & $\begin{array}{r}10 \\
.01 \\
.00\end{array}$ & $\begin{array}{l}11 \\
.0 \\
.00\end{array}$ & $\begin{array}{c}\text { Hardness as } \mathrm{CaCO}_{3}: \\
\text { Total .................. } \\
\text { Noncarbonate...... }\end{array}$ & $\begin{array}{r}10 \\
5\end{array}$ & $\begin{array}{l}36 \\
20\end{array}$ \\
\hline 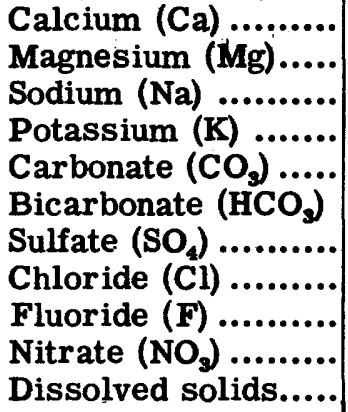 & $\begin{array}{l}2.4 \\
1.0 \\
1.4 \\
1.5 \\
0 \\
6 \\
6.0 \\
3.5 \\
.0 \\
1.1 \\
35\end{array}$ & $\begin{array}{l}12 \\
1.5 \\
2.0 \\
1.6 \\
0 \\
20 \\
16 \\
6.6 \\
.0 \\
.3\end{array}$ & $\begin{array}{l}\text { Color ................... } \\
\text { pH .......................... } \\
\text { Specific conductance } \\
\text { (micromhos at } \\
25 \text { C. ) ................ } \\
\text { Turbidity ............. } \\
\text { Temperature (F.)... } \\
\text { Date of collection ... }\end{array}$ & $\begin{array}{r}42.9 \\
1.0 \\
-- \\
\text { Mar.20, } \\
1951\end{array}$ & $\begin{array}{r}95.7 \\
.9 \\
-- \\
\text { Mar. 20, } \\
1951\end{array}$ \\
\hline
\end{tabular}

ampounding Reservoir

bSurface supplies, only 


\section{BALTIMORE}

(Population, 949, 708)

Ownership: Municipal; supplies a large population in the Metropolitan District of Baltimore County. Total population supplied, about 1, 163, 000 .

Source: Gunpowder River impounded in Lock Raven Reservoir. North Branch of Patapsco River, (to be tapped about the middle of 1951 adding about 40, 000, 000 gpd to the present supply), auxiliary supply.

Treatment: Plain sedimentation, prechlorination to $0.5 \mathrm{ppm}$ of free available chlorine through filters, coagulation with alum, sedimentation, rapid sand filtration, and adjustment of $\mathrm{pH}$ to 7.8 with lime.

Rated capacity of treatment plant: $240,000,000 \mathrm{gpd}$.

Raw-water storage: 43, 000,000, 000 gal.

Finished-water storage: Filtered water reservoirs, elevated tanks, standpipes. Total 773, 500, 000 gal.

The Metropolitan District of Baltimore County borders Baltimore City on the west, north, and east and the water supply for the District is obtained from the city supply. After the distribution system is installed, it is turned over to the city for maintenance and operation and forms an integral part of the city system.

It is proposed to construct eventually an impounding dam on the North Branch of Patapsco River and another treatment plant.

\section{ANALYSES}

(Analyses, in parts per million, by Monte Bello Laboratory, Baltimore, Maryland)

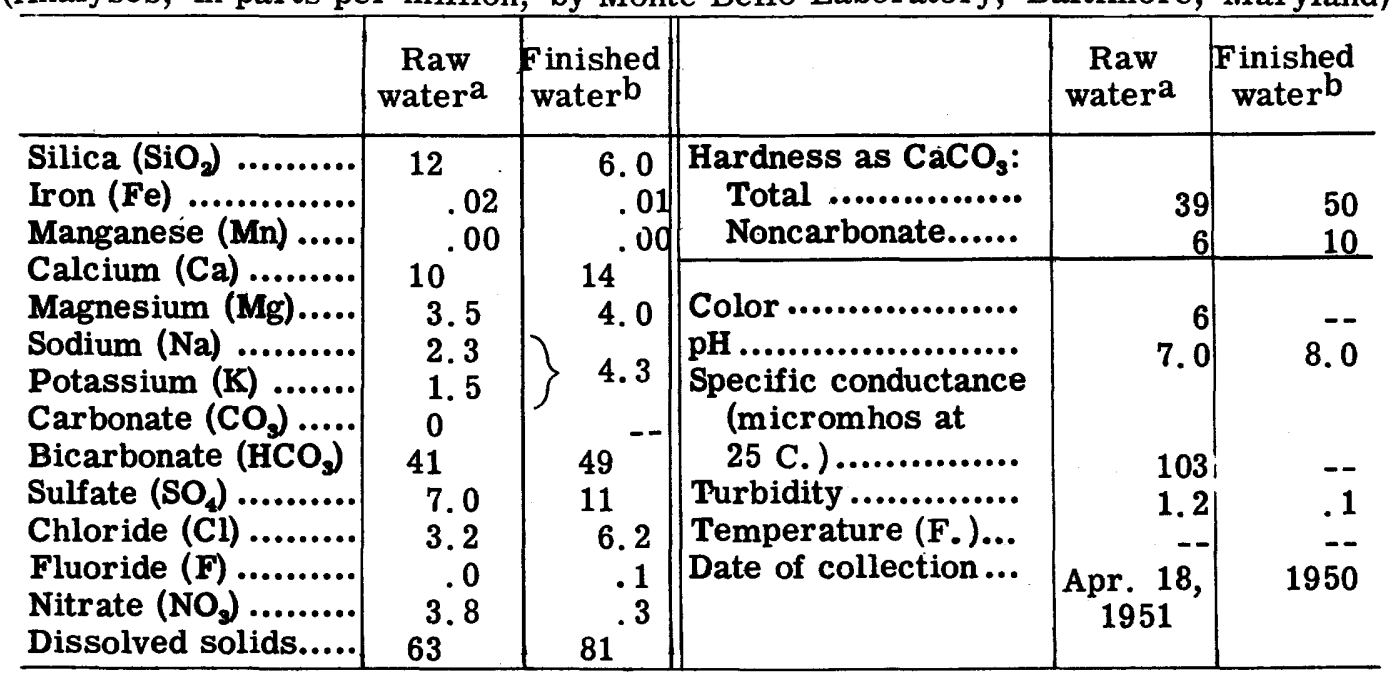

Regular determinations at treatment plant, 1950

\begin{tabular}{|c|c|c|c|c|c|c|c|c|c|c|c|c|}
\hline & \multicolumn{3}{|c|}{$\begin{array}{c}\text { Alkalinity } \\
\text { as } \mathrm{CaCO}_{3} \\
\text { (ppm) }\end{array}$} & \multicolumn{3}{|c|}{ pH } & \multicolumn{3}{|c|}{$\begin{array}{c}\text { Hardness } \\
\text { as } \mathrm{CaCO}_{3} \\
(\mathrm{ppm})\end{array}$} & \multicolumn{3}{|c|}{ Turbidity } \\
\hline & Av & $\operatorname{Max}$ & Min & Av & $\operatorname{Max}$ & Min & Av & Max & Min & Av & $\operatorname{Max}$ & Min \\
\hline $\begin{array}{l}\text { Raw water.......... } \\
\text { Finished water... }\end{array}$ & $\begin{array}{l}37 \\
40\end{array}$ & $\begin{array}{l}44 \\
52\end{array}$ & $\begin{array}{l}33 \\
35\end{array}$ & $\begin{array}{l}7.2 \\
8.0\end{array}$ & $\begin{array}{l}7.4 \\
8.4\end{array}$ & $\begin{array}{l}6.8 \\
7.3\end{array}$ & $\begin{array}{l}40 \\
52\end{array}$ & $\begin{array}{l}46 \\
63\end{array}$ & $\begin{array}{l}37 \\
45\end{array}$ & 5 & $\left|\begin{array}{l}30 \\
.2\end{array}\right|$ & 3 \\
\hline
\end{tabular}

${ }^{a}$ Reservoir. Analysis by U. S. Geological Survey.

b Averages of analyses of monthly composites of daily samples. 


\section{COLLEGE PARK}

(Population, 11, 170)

Ownership: Supplied by Washington Suburban Sanitary Commission. (See Hyattsville.)

\section{CUMBERLAND \\ (Population, 37,679)}

Ownership: Municipal; (Evitts Creek Water Company) supplies also Cresaptown and La Vale, Maryland; Ridgeley and Wiley Ford, West Virginia. Total population supplied, about 42,100 .

Source: Evitts Creek impounded in two lakes, Lake Gordon and Koon Lake.

Treatment: Coagulation with alum, sedimentation, rapid (anthrafilt) filtration, chlorination, and ammoniation.

Rated capacity of treatment plant: $12,000,000 \mathrm{gpd}$.

Raw-water storage: Koon Lake, 2, 300, 000, 000 gal; Lake Gordon 1, 330, 000, 000 gal.

Finished-water storage: Fort Hill reservoir, 3, 750, 000 gal; Ridgedale reservoir, 7, 500, 000 gal.

The treatment plant is located at Koon Lake about 8 miles northeast of the city.

The finished water flows by gravity through two 36 -in. reinforced concrete conduits from the treatment plant to the finished water reservoirs, thence to the distribution system.

\section{ANALYSES}

(Analyses, in parts per million, by U. S. Geological Survey)

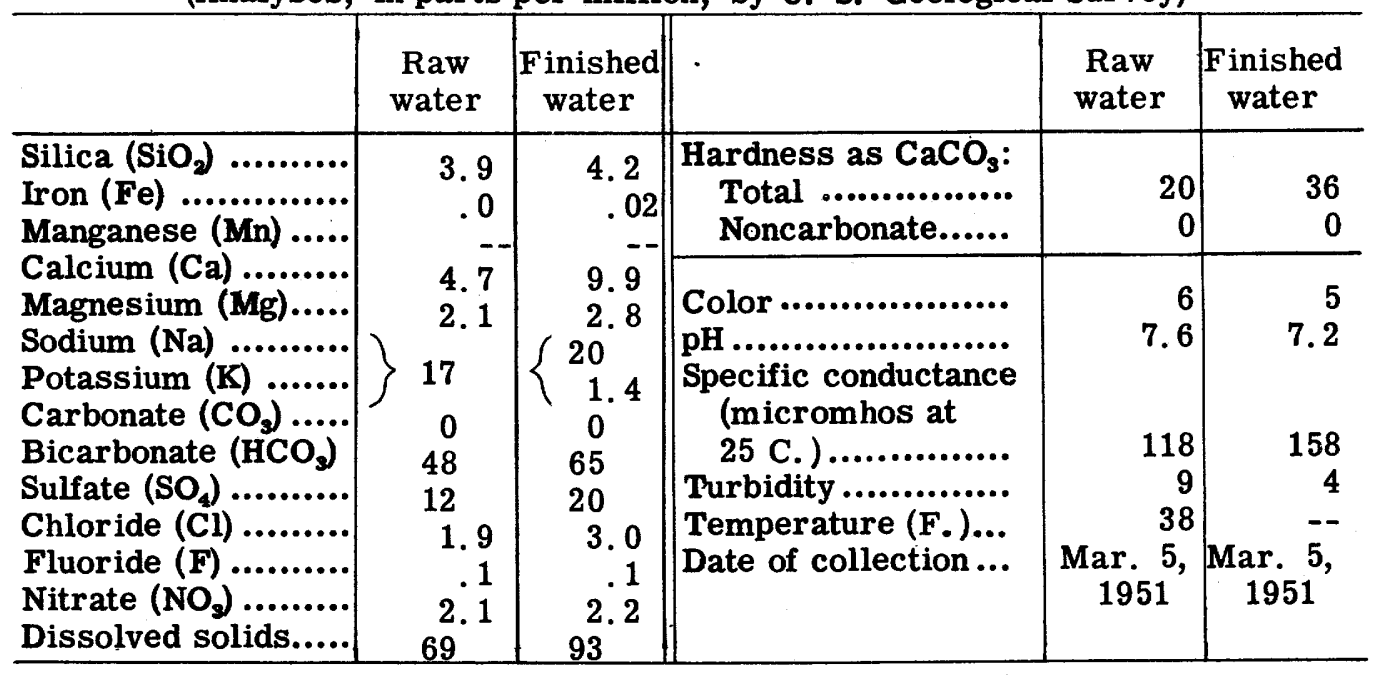


FREDERICK

(Population, 18, 142)

Ownership:_Municipal; a small population is supplied outside the city limits. Total population supplied, about 18,200.

Source: Tuscarora and Fishing Creeks about 84 percent of supply; Linganore Creek, about 16 percent of supply. Fishing Creek (impounded) is the main source of supply. Linganore Creek water is used to supplement the regular supply at times of peak load or when necessary.

Treatment: Main supply: Chlorination and ammoniation. Linganore Creek water: coagulation with alum and lime, carbon, breakpoint chlorination, sedimentation, rapid sand filtration, and postchlorination.

Rated capacity of treatment plant: 6,000,000 gpd.

Raw-water storage: Fishing creek reservoir, 77,000,000 gal.

Finished-water storage: Clear wells and elevated tanks, 1,000,000 gal.

Fishing Creek reservoir is located about 10 miles northwest of the city, above the village of Mountain Dale. The water from Tuscarora Creek reservoir enters the conduit from Fishing Creek reservoir near Yellow Springs. Thus the water from these two sources is mixed before entering the city. The treatment plant for the Linganore Creek supply is about 3.5 miles east of the city.

\section{ANALYSES}

(Analyses, in parts per million, by U. S. Geological Survey)

\begin{tabular}{|c|c|c|c|}
\hline & $\begin{array}{c}\text { Fishing Creek } \\
\text { reservoir } \\
\text { (raw water) } \\
\end{array}$ & Fishing Creek & $\begin{array}{l}\text { Finished water } \\
\text { Linganore Creek }\end{array}$ \\
\hline Silica $\left(\mathrm{SiO}_{2}\right) \ldots$ & 3.0 & 0.6 & 5.7 \\
\hline Iron $(\mathrm{Fe})$............................. & .01 & .00 & .01 \\
\hline Manganese (Mn) & & & \\
\hline Calcium (Ca) .......... & .5 & .7 & \\
\hline Magnesium (Mg).... & .1 & .2 & 3.4 \\
\hline Sodium (Na).......... & 2.6 & & \\
\hline Potassium (K) $\ldots \ldots \ldots \ldots \ldots \ldots \ldots . . . . .$. & .3 & 2.1 & 6.8 \\
\hline Carbonate $\left(\mathrm{CO}_{3}\right) \ldots \ldots \ldots \ldots \ldots \ldots . . . . . . . . . . .$. & 0 & 0 & 0 \\
\hline Sulfate $\left(\mathrm{SO}_{4}\right) \ldots \ldots \ldots \ldots \ldots \ldots$ & $\begin{array}{l}3.5 \\
2.4\end{array}$ & $\begin{array}{l}4 \\
1.5\end{array}$ & $\begin{array}{l}26 \\
20\end{array}$ \\
\hline Chloride (Cl) $\ldots . \ldots \ldots \ldots \ldots \ldots \ldots \ldots$ & 1.4 & 1. 5 & 6.2 \\
\hline Fluoride $(F)$............................ & .0 & .0 & .1 \\
\hline Nitrate $\left(\mathrm{NO}_{3}\right), \ldots \ldots \ldots \ldots \ldots \ldots \ldots \ldots$ & .1 & .2 & 6.5 \\
\hline Dissolved solids $\ldots . \ldots \ldots \ldots \ldots \ldots \ldots$ & 12 & 12 & 78 \\
\hline $\begin{array}{l}\text { Hardness as } \mathrm{CaCO}_{3} \text { : } \\
\text { Total .................. }\end{array}$ & 2 & 3 & 41 \\
\hline Noncarbonate ... & 0 & 0 & 20 \\
\hline Color & & & \\
\hline pH.................. & 6. 1 & 5.7 & 7. 0 \\
\hline Specific conduct & & & \\
\hline & 14.5 & 14.5 & 131 \\
\hline Turbidity ..................... & & & 2 \\
\hline Tem & & & -- \\
\hline Date & Mar. 6, 1951 & Oct. 16,1951 & Mar. 6, 1951 \\
\hline
\end{tabular}

Regular determinations at treatment plant, 1950a

\begin{tabular}{|c|c|c|c|c|c|c|c|c|c|c|c|c|}
\hline & \multicolumn{3}{|c|}{$\begin{array}{l}\text { Alkalinity } \\
\text { as } \mathrm{CaCO} \\
\text { (ppm) }\end{array}$} & \multicolumn{3}{|c|}{$\mathrm{pH}$} & \multicolumn{3}{|c|}{$\begin{array}{l}\text { Hardness } \\
\text { as } \mathrm{CaCO} \\
\text { (ppm) }\end{array}$} & \multicolumn{3}{|c|}{ Turbidity } \\
\hline & Av & Max & Min & Av & Max & Min & Av & $\operatorname{Max}$ & Min & Av & Max & Min \\
\hline $\begin{array}{l}\text { Raw water......... } \\
\text { Finished water... }\end{array}$ & $\begin{array}{l}46 \\
34\end{array}$ & $\begin{array}{l}61 \\
45\end{array}$ & $\begin{array}{l}17 \\
23\end{array}$ & $\begin{array}{l}7.1 \\
6.6\end{array}$ & \begin{tabular}{|l|}
8.6 \\
7.0 \\
\end{tabular} & \begin{tabular}{|l|}
6.9 \\
6.2
\end{tabular} & $\begin{array}{l}52 \\
53\end{array}$ & \begin{tabular}{|l|}
66 \\
68 \\
\end{tabular} & $\begin{array}{l}39 \\
44 \\
\end{array}$ & $\begin{array}{r}15 \\
5\end{array}$ & $\begin{array}{r}1000 \\
5\end{array}$ & $\begin{array}{l}5 \\
5\end{array}$ \\
\hline
\end{tabular}

aLinganore Creek. 


\section{HAGERSTOWN}

(Population, 32, 260)

Ownership: Municipal; supplies also Williamsport, Funkstown, Smithburg, and a number of people outside the city limits. Total population supplied, about 45,000 .

Source: Potomac River, 2/3 of supply; mountain supply consisting of Raven Rock and Warner Hollow Creeks, impounded, 1/3 of supply.

Treatment: Potomac River supply: Plain sedimentation, prechlorination, coagulation with alum, occasionally activated carbon, sedimentation, rapid sand filtration, addition of lime for adjustment of $\mathrm{pH}$. Mountain supply: Chlorination.

Rated capacity of treatment plant: $10,000,000 \mathrm{gpd}$.

Raw-water storage: Potomac River supply: 2 settling basins, 5, 000, 000 gal each. Mountain supply: reservoir, 120,000,000 gal.

Finished-water storage: Equalizing reservoirs and elevated tanks, 11, 500, 000 gal.

The two sources of supply are interconnected so that the consumers may be furnished with river, mountain streams, or the mixed water. There is considerable variation in composition of the water from both supplies throughout the year, but the water of the mountain supply is low in mineral content and hardness at all times.

Fluoridation with sodium silicofluoride was begun Nov. 20, 1951 of the Mountain supply and Dec. 4, 1951 of the Potomac River supply. An average content of about $1.1 \mathrm{ppm}$ of fluoride is maintained in the supplies.

\section{ANALYSES}

(Analyses, in parts per million, by U. S. Geological Survey)

\begin{tabular}{|c|c|c|c|}
\hline & $\begin{array}{c}\text { Potomac River } \\
\text { (raw water) }\end{array}$ & $\begin{array}{l}\text { Potomac River } \\
\text { (finished water) }\end{array}$ & $\begin{array}{l}\text { Mountain Supply } \\
\text { (city tap) }\end{array}$ \\
\hline 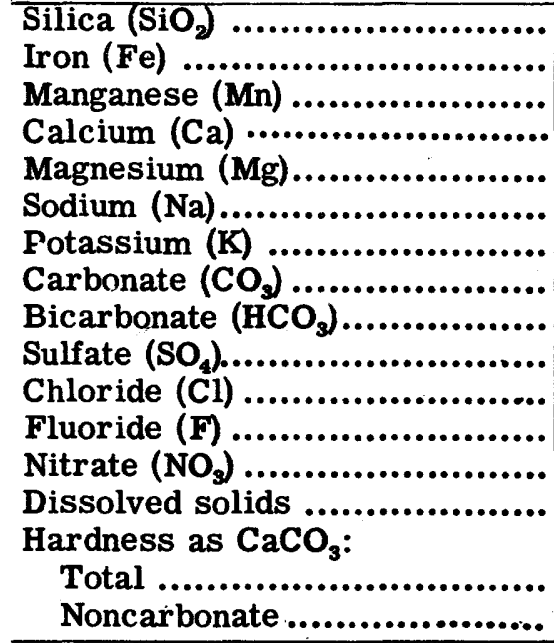 & $\begin{array}{c}4.8 \\
.03 \\
11 \\
5.0 \\
13 \\
0 \\
42 \\
=35 \\
2.9 \\
.0 \\
1.5 \\
97 \\
48 \\
14\end{array}$ & $\begin{array}{c}5.4 \\
.01 \\
17 \\
3.8 \\
15 \\
1.1 \\
0 \\
45 \\
44 \\
4.8 \\
.0 \\
1.6 \\
116 \\
58 \\
21\end{array}$ & $\begin{array}{c}0 \\
13 \\
9.2 \\
4.0 \\
.2 \\
1.6 \\
45 \\
\\
17 \\
7\end{array}$ \\
\hline 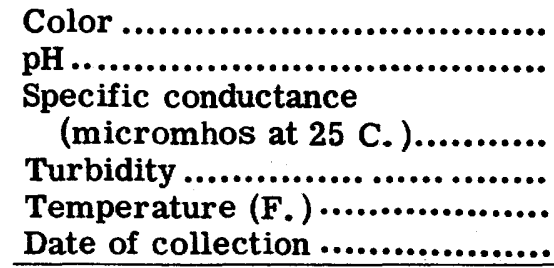 & $\begin{array}{r}6 \\
7.3 \\
161 \\
9 \\
\text { Mar. 6, } 1951 \\
\end{array}$ & $\begin{array}{r}3 \\
7.8 \\
193 \\
2 \\
\text { Mar. } 6,19 \overline{5} \\
\end{array}$ & $\begin{array}{r}4 \\
7.0 \\
59.5 \\
5\end{array}$ \\
\hline
\end{tabular}


HAGERSTOWN--Continued

Regular determinations at treatment plant, 1950

\begin{tabular}{l|r|r|r|r|r|r|r|r|r|r|r|r}
\hline & \multicolumn{3}{|c|}{$\begin{array}{c}\text { Alkalinity } \\
\text { as CaCO } \\
\text { (ppm) }\end{array}$} & \multicolumn{3}{c}{ pH } & \multicolumn{3}{c|}{$\begin{array}{c}\text { Hardness } \\
\text { as CaCO } \\
\text { (ppm) }\end{array}$} & \multicolumn{3}{c}{ Turbidity } \\
\cline { 2 - 14 } & Av & Max & Min & Av & Max & Min & Av & Max & Min & Av & Max & Min \\
\hline $\begin{array}{l}\text { Potomac River } \\
\text { Raw water.... }\end{array}$ & 65 & 100 & 20 & 7.3 & 7.6 & 6.6 & 80 & 130 & 25 & 20 & 1600 & 6 \\
\hline $\begin{array}{l}\text { Potomac River } \\
\text { Finished water }\end{array}$ & 65 & 100 & 10 & 7.7 & 8.2 & 7.2 & 85 & 140 & 30 & 5 & 10 & .2 \\
\hline $\begin{array}{l}\text { Mt. Supply } \\
\text { Raw water.... }\end{array}$ & 15 & 25 & 10 & 6.5 & 6.9 & 6.3 & 20 & 25 & 5 & 8 & 15 & 5 \\
\hline
\end{tabular}




\author{
HYATTSVILLE \\ (Population, 12,300)
}

Ownership: Washington Suburban Sanitary Commission, a public body incorporated by the Maryland General Assembly, serving metropolitan areas of about 200 square miles in Montgomery and Prince Georges Counties. Supplies Bethesda, Bladensburg, Brentwood, Capitol Heights, Chevy Chase, College Park, Colmar Manor, Fairmount Heights, Gaithersburg, Mount Rainier, Riverdale, Silver Spring, Takoma Park, Wheaton and other communities. Total population supplied, about 280,000 .

Source: Patuxent River and the Northwest Branch of the Anacostia River, both impounded. Water from the Patuxent River can be pumped into the Northwest Branch of the Anacostia River when needed.

Treatment: (Both plants): Prechlorination, coagulation with alum (and lime or sodium aluminate at times), sedimentation, rapid sand filtration, postchlorination or dechlorination as may be necessary, and addition of lime for adjustment of $\mathrm{pH}$.

Rated capacity of treatment plants: Burnt Mills Plant (N. W. Branch of Anacostia River), 10,000, 000 gpd; Willis School Plant (Patuxent River), 26, 700, 000 gpd.

Raw-water storage: Northwest Branch Anacostia River reservoir, 50, 000, 000 gal; Patuxent River reservoir, 6,118,000,000 gal.

Finished-water storage: Reservoirs, standpipes and elevated tanks for both high and low service, $38,175,000 \mathrm{gal}$.

The Northwest Branch of Anacostia River is the main source of supply for the high service area chiefly in Montgomery County. The dam impounding the river and the Burnt Mills treatment plant are located on Colesville Road near Silver Spring, Montgomery County. During dry seasons when the flow of the Northwest Branch is inadequate, water from the Patuxent River is pumped into the Northwest Branch from the Mink Hollow Pumping station near Ashton, about 10 miles above the Burnt Mills treatment plant. High service connections to the supply of District of Columbia total 6,000,000 gpd in case of emergency.

The source of supply for the low service is the Patuxent River, impounded by the Brighton Dam 14 miles north of the northernmost tip of Washington. The water is pumped from the river at the Rocky Gorge pumping station 13 miles below the dam to the Willis School treatment plant 2 miles west of Laurel, Prince Georges County. The finished water flows by gravity to the low service area. Connections in cases of emergency to the supply of the District of Columbia to this service area total 3,200,000 gpd.

The treated supplies of the two sources are further interconnected so that most of the capacity available for the high service in one zone is also available for the low service in the other zone. 
HYATTSVILLE--Continued

ANALYSES

(Analyses, in parts per million, by U. S. Geological Survey)

\begin{tabular}{|c|c|c|c|}
\hline & \multicolumn{2}{|c|}{ Patuxent River } & \multirow{2}{*}{$\begin{array}{c}\text { Finished } \\
\text { water c }\end{array}$} \\
\hline & Raw water a & Finished water & \\
\hline 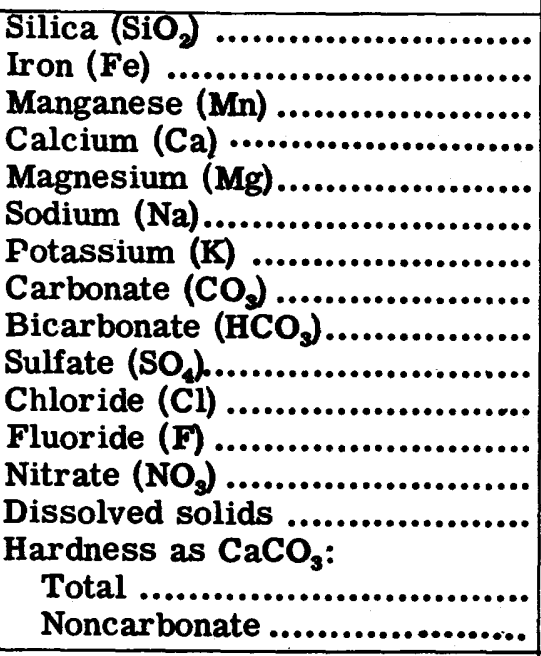 & $\begin{array}{c}9.7 \\
.02 \\
.00 \\
4.0 \\
1.1 \\
2.6 \\
1.2 \\
0 \\
15 \\
4.0 \\
3.2 \\
.0 \\
1.9 \\
37 \\
14 \\
2\end{array}$ & $\begin{array}{c}8.9 \\
.09 \\
.00 \\
8.8 \\
1.6 \\
2.9 \\
1.1 \\
0 \\
18 \\
8.5 \\
7.2 \\
.0 \\
2.4 \\
56 \\
\\
29 \\
14 \\
\end{array}$ & $\begin{array}{c}14 \\
.01 \\
.00 \\
10 \\
1.7 \\
4.6 \\
.9 \\
0 \\
26 \\
10 \\
7.8 \\
.0 \\
1.2 \\
68 \\
32 \\
11\end{array}$ \\
\hline 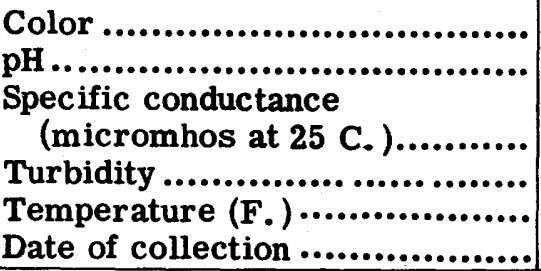 & $\begin{array}{r}2 \\
6.8 \\
54.6 \\
.9 \\
\text { Apr. } 9,1951\end{array}$ & $\begin{array}{r}4 \\
7.4 \\
84.9 \\
1.0 \\
\text { Apr. 9, } 195 \overline{1}\end{array}$ & $\begin{array}{r}2 \\
7.4 \\
102 \\
.5 \\
\text { Apr. } 9,19 \overline{51}\end{array}$ \\
\hline
\end{tabular}

Regular determinations at treatment plant, $1950^{\mathrm{d}}$

\begin{tabular}{|c|c|c|c|c|c|c|c|c|c|c|c|c|}
\hline & \multicolumn{3}{|c|}{$\begin{array}{l}\text { Alkalinity } \\
\text { as CaCO } \\
\text { (ppm) }\end{array}$} & \multicolumn{3}{|c|}{ pH } & \multicolumn{3}{|c|}{$\begin{array}{c}\text { Hardness } \\
\text { as } \mathrm{CaCO} \\
\text { (ppm) }\end{array}$} & \multicolumn{3}{|c|}{ Turbidity } \\
\hline & Av & $\operatorname{Max}$ & Min & Av & Max & Min & Av & Max & Min & Av & $\operatorname{Max}$ & Min \\
\hline $\begin{array}{l}\text { Raw water......... } \\
\text { Finished water... }\end{array}$ & $\begin{array}{l}14 \\
19\end{array}$ & $\begin{array}{l}22 \\
25\end{array}$ & $\begin{array}{r}8 \\
12\end{array}$ & $\begin{array}{l}7.0 \\
8.0\end{array}$ & $\begin{array}{l}7.4 \\
8.7\end{array}$ & $\begin{array}{l}6.0 \\
7.0\end{array}$ & $\begin{array}{l}18 \\
33\end{array}$ & $\begin{array}{l}25 \\
50\end{array}$ & $\begin{array}{l}15 \\
28\end{array}$ & $\begin{array}{r}60 \\
.20\end{array}$ & $\begin{array}{l}6000 \\
1.30\end{array}$ & $\begin{array}{r}10 \\
.10\end{array}$ \\
\hline $\begin{array}{l}\text { Raw water } \mathrm{e} . . . \\
\text { Finished water } \mathrm{e}\end{array}$ & $\begin{array}{l}20 \\
24\end{array}$ & $\begin{array}{l}26 \\
33\end{array}$ & $\begin{array}{l}10 \\
10\end{array}$ & $\begin{array}{l}6.9 \\
8.0\end{array}$ & $\begin{array}{l}7.6 \\
8.7\end{array}$ & $\begin{array}{l}6.1 \\
6.8\end{array}$ & $\begin{array}{l}22 \\
39\end{array}$ & $\begin{array}{l}35 \\
52\end{array}$ & $\begin{array}{l}16 \\
31\end{array}$ & $\begin{array}{r}70 \\
.23\end{array}$ & $\begin{array}{l}4000 \\
1.60\end{array}$ & $\begin{array}{r}10 \\
.10\end{array}$ \\
\hline
\end{tabular}

a At Willis School treatment plant.

b City tap, Hyattsville.

c N. W. Branch Anacostia River, at Burnt Mills treatment plant.

d Willis School treatment plant.

e Burnt Mills treatment plant.

MOUNT RAINIER

(Population, 10, 989)

Ownership: Supplied by Washington Suburban Sanitary Commision. (See Hyattsville.) 


\section{SALISBURY}

(Population, 15, 141)

Ownership: Municipal.

Source: Ten wells (1 to 10) 58, 57, 43, 48, 61, 65, 62, 66, 60, and $60 \mathrm{ft}$ deep. Emergency supply, Schumacher Pond. All the wells are equipped with turbine pumps except wells 1 and 3 which are suction lift. Wells 1 to 5 are reported to yield a total of about 2,000 gpm. Wells 2, 9, and 10 furnished the greater part of the supply during the winter of 1950-51.

Treatment: Aeration (coke beds), addition of lime to raise $\mathrm{pH}$, chlorination, and ammoniation.

Rated capacity of treatment plant: 5, 500,000 gpd.

Raw-water storage: Underground reservoir at plant, 500, 000 gal.

Finished-water storage: 2 elevated tanks, 200,000 and 300,000 gal; 1 standpipe, 85,000 gal.

The wells are in the city park area and are operated by remote control (except wells 9 and 10 not equipped at present) and pump to the reservoir at the treatment plant. All of the wells are not pumped at the same time, and none continuously. Enough wells are used to take care of daily requirements.

\section{ANALYSES}

(Analyses, in parts per million, by U. S. Geological Survey)

\begin{tabular}{|c|c|c|c|}
\hline & $\begin{array}{c}\text { Well } 2 \\
\text { (raw water) }\end{array}$ & $\begin{array}{l}\text { Well } 10 \\
\text { (raw water) }\end{array}$ & $\begin{array}{c}\text { Wells } 2,4,5, \\
7,8 \\
\text { (finished water) }\end{array}$ \\
\hline 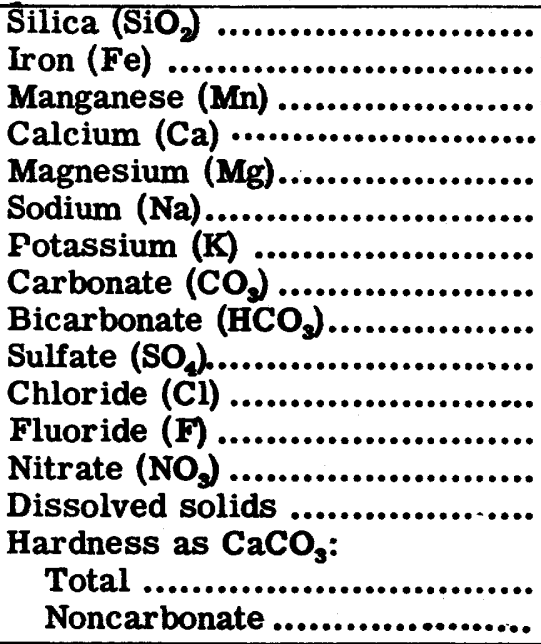 & \begin{tabular}{c|}
19 \\
1.0 \\
.05 \\
4.6 \\
1.4 \\
7.8 \\
1.9 \\
0 \\
12 \\
3.2 \\
7.5 \\
.1 \\
13 \\
70 \\
17 \\
7
\end{tabular} & $\begin{array}{c}18 \\
.02 \\
.00 \\
2.9 \\
.5 \\
9.7 \\
0 \\
13 \\
3.5 \\
6.8 \\
.0 \\
8.0 \\
60\end{array}$ & $\left\{\begin{array}{c}19 \\
.35 \\
.01 \\
7.0 \\
.6 \\
10 \\
1.7 \\
0 \\
22 \\
2.0 \\
9.0 \\
.0 \\
12 \\
81 \\
20 \\
2\end{array}\right.$ \\
\hline 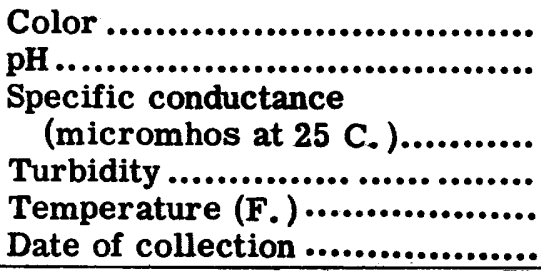 & Mar. 3, 1948 & Mar. 19,1951 & $\begin{array}{r}3 \\
7.1 \\
104 \\
11 \\
\text { Mar. } 19,1951 \\
\end{array}$ \\
\hline 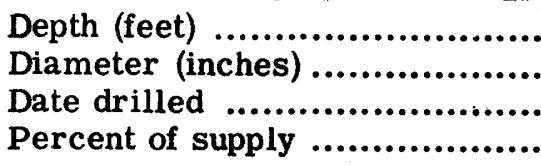 & $\begin{array}{r}57 \\
24 \\
1925 \\
--\end{array}$ & $\begin{array}{r}60 \\
18 \\
1949\end{array}$ & \\
\hline
\end{tabular}




\section{ALBEMARLE \\ (Population, 11, 798)}

Ownership: Municipal; also supplies suburban districts. Total population supplied, about 12,300 .

Source: Yadkin River. The intake is about 7 miles northwest of Badin.

Treatment: Coagulation with alum, soda ash, chlorination, Calgon, aeration, sedimentation, and rapid sand filtration.

Rated capacity of treatment plant: 4,000, $000 \mathrm{gpd}$.

Raw-water storage: 1 reservoir, 30,000,000 gal.

Finished-water storage: 2 concrete reservoirs, each 1, 000,000 gal; elevated tank, 100, 000 gal; standpipe, 500, 000 gal.

\section{ANALYSES}

(Analyses, in parts per million, by U. S. Geological Survey)

\begin{tabular}{|c|c|c|c|c|c|}
\hline & $\begin{array}{c}\text { Raw } \\
\text { water }\end{array}$ & $\begin{array}{c}\text { Finished } \\
\text { water }\end{array}$ & & $\begin{array}{l}\text { Raw } \\
\text { water }\end{array}$ & $\begin{array}{l}\text { Finished } \\
\text { water }\end{array}$ \\
\hline $\begin{array}{l}\text { Silica }\left(\mathrm{SiO}_{2}\right) \\
\text { Iron }(\mathrm{Fe}) \ldots \ldots \ldots \ldots \\
\text { Manganese }(\mathrm{Mn}) \ldots \ldots . . . .\end{array}$ & $\begin{array}{r}11 \\
.09 \\
.00\end{array}$ & $\begin{array}{l}9.7 \\
.05 \\
.00\end{array}$ & $\begin{array}{r}\text { Hardness as } \mathrm{CaCO}_{3}: \\
\text { Total } \ldots . . . . . . . . . . . . . . \\
\text { Noncarbonate...... }\end{array}$ & $\begin{array}{r}18 \\
0\end{array}$ & $\begin{array}{r}19 \\
0\end{array}$ \\
\hline $\begin{array}{l}\text { Calcium }(\mathrm{Ca}) . . . . . . . \\
\text { Magnesium }(\mathrm{Mg}) . . . .\end{array}$ & $\begin{array}{l}4.4 \\
1.7\end{array}$ & $\begin{array}{l}4.9 \\
1.7\end{array}$ & Color ..................... & 3 & 4 \\
\hline $\begin{array}{l}\text { Sodium (Na) ........... } \\
\text { Potassium (K) ....... }\end{array}$ & 5.8 & 16 & pH ....................... & 7.0 & .6 .9 \\
\hline $\begin{array}{l}\text { Carbonate }\left(\mathrm{CO}_{3}\right) \ldots . . . \\
\text { Bicarbonate }\left(\mathrm{HCO}_{3}\right)\end{array}$ & $\begin{array}{r}0 \\
25\end{array}$ & $\begin{array}{r}0 \\
32\end{array}$ & 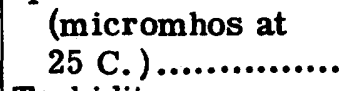 & 63.8 & 118 \\
\hline Sulfate $\left(\mathrm{SO}_{4}\right) \ldots \ldots \ldots$ & 4.4 & 20 & Turbidity ............... & -- & -- \\
\hline Chloride (Cl) ........... & 3.2 & 4.8 & Temperature (F.).... & & -- \\
\hline $\begin{array}{l}\text { Fluoride }(F) \ldots \ldots \ldots \ldots \\
\text { Nitrate }\left(\mathrm{NO}_{2}\right) \ldots \ldots \ldots . . \\
\text { Dissolved solids...... }\end{array}$ & $46^{.7}$ &.$^{.4}$ & Date of collection... & $\begin{array}{c}\text { May 6, } \\
1951\end{array}$ & $\begin{array}{c}\text { May 6, } \\
1951\end{array}$ \\
\hline
\end{tabular}

Regular determinations at treatment plant, 1950

\begin{tabular}{|c|c|c|c|c|c|c|c|c|c|c|c|c|}
\hline & \multicolumn{3}{|c|}{$\begin{array}{c}\text { Alkalinity } \\
\text { as } \mathrm{CaCO} \\
\text { (ppm) }\end{array}$} & \multicolumn{3}{|c|}{ pH } & \multicolumn{3}{|c|}{$\begin{array}{l}\text { Hardness } \\
\text { as } \mathrm{CaCO}_{3} \\
\text { (ppm) }\end{array}$} & \multicolumn{3}{|c|}{ Turbidity } \\
\hline & Av & Max & Min & Av & Max & Min & Av & Max & Min & Av & $\operatorname{Max}$ & Min \\
\hline $\begin{array}{l}\text { Raw water......... } \\
\text { Finished water... }\end{array}$ & \begin{tabular}{l|}
20 \\
22
\end{tabular} & \begin{tabular}{|l|}
22 \\
24 \\
\end{tabular} & $\begin{array}{l}18 \\
20\end{array}$ & $\begin{array}{l}7.0 \\
6.8\end{array}$ & $\begin{array}{l}7.1 \\
6.9\end{array}$ & $\begin{array}{l}6.9 \\
6.8\end{array}$ & $\begin{array}{l}20 \\
20\end{array}$ & \begin{tabular}{|l|}
22 \\
22 \\
\end{tabular} & $\begin{array}{l}18 \\
18\end{array}$ & $\begin{array}{r}20 \\
0\end{array}$ & $\begin{array}{r}400 \\
0\end{array}$ & $\begin{array}{l}5 \\
0\end{array}$ \\
\hline
\end{tabular}


ASHEVILLE

(Population, 53, 000)

Ownership: Municipal; also supplies a number of communities in the Buncombe County Water System. Total population supplied, about 73,000.

Source: Beetree Creek (impounded) and Right and Left Forks of the North Fork of the Swannanoa River (impounded). The intakes are about 12 miles east of the city.

Treatment: Chlorination.

Raw-water storage: Beetree Reservoir, 500,000, 000 gal.

Finished-water storage: Beaucatcher Reservoir, 5,000,000 gal; White Fawn Reservoir, 11,300,000 gal; Standpipe, 300,000 gal.

\section{ANALYSES}

(Analyses, in parts per million, by U. S. Geological Survey)

\begin{tabular}{|c|c|c|c|c|}
\hline & $\begin{array}{c}\text { Beetree } \\
\text { Reservoir }\end{array}$ & $\begin{array}{c}\text { Beetree } \\
\text { Reservoir }^{b}\end{array}$ & $\begin{array}{l}\text { North Fork } \\
\text { Swannanoa } \\
\text { River }\end{array}$ & $\begin{array}{c}\text { North Fork } \\
\text { Swannanoa } \\
\text { Riverb }\end{array}$ \\
\hline Silica $\left(\mathrm{SiO}_{2}\right)$.. & 8.3 & 7.4 & 5.6 & 6.2 \\
\hline Iron (Fe) $\ldots . . \ldots \ldots \ldots \ldots \ldots \ldots$ & .02 & .02 & .03 & .04 \\
\hline Manganese $(\mathrm{Mn})$................. & .00 & .00 & .00 & .00 \\
\hline Calcium (Ca) ..................... & 1.8 & 1.6 & 1.0 & 1.6 \\
\hline Magnesium (Mg) .......... & .7 & .8 & .4 & .3 \\
\hline $\begin{array}{l}\text { Sodium (Na) } \ldots \ldots \ldots \ldots \ldots \ldots \ldots \ldots \\
\text { Potassium (K) }\end{array}$ & 2.3 & 1.1 & 2.4 & 1.7 \\
\hline Carbonate $\left(\mathrm{CO}_{3}\right) \ldots \ldots \ldots \ldots . . . . .$. & 0 & 0 & 0 & 0 \\
\hline Bicarbonate $\left(\mathrm{HCO}_{2}\right)$.............. & 7 & 6 & 4 & 7 \\
\hline Sulfate $\left(\mathrm{SO}_{4}\right) \ldots \ldots \ldots \ldots \ldots \ldots$ & 2.9 & 2.6 & 2.8 & 1.5 \\
\hline 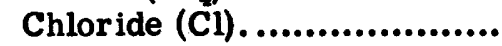 & 2.5 & 1.2 & 1.9 & 1.1 \\
\hline Fluoride $(F)$.................... & .0 & .0 & .0 & .0 \\
\hline Nitrate $\left(\mathrm{NO}_{3}\right)$..................... & .1 & .4 & .6 & .2 \\
\hline Dissolved solids ................ & 22 & 20 & 17. & 16 \\
\hline Hardness as $\mathrm{CaCO}_{\mathbf{3}}$ : & & & & \\
\hline Total $\ldots \ldots \ldots \ldots \ldots \ldots \ldots \ldots$ & 7 & 7 & 4 & $\mathbf{5}$ \\
\hline Noncarbonate ................. & 2 & 2 & 1 & 0 \\
\hline Color................ & 3 & 3 & & 4 \\
\hline pH $\ldots \ldots \ldots \ldots \ldots \ldots \ldots \ldots \ldots \ldots . . . . . . .$. & 5.8 & 6.2 & 5.9 & 6.7 \\
\hline Specific conductance & & & & \\
\hline (micromhos at 25 C.) $\ldots . .$. & 24.0 & 24.6 & 15.2 & 14.0 \\
\hline Turbidity $\ldots . . . \ldots \ldots \ldots \ldots \ldots \ldots \ldots$ & -- & -- & -- & -- \\
\hline Temperature (F.) .............. & -- & -- & 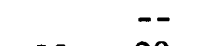 & -- \\
\hline Date of collection ................ & $\begin{array}{c}\text { Apr. } 12, \\
1950\end{array}$ & $\underset{1950}{\text { Apr. } 12,}$ & $\begin{array}{c}\text { Mar. 28, } \\
1950\end{array}$ & $\begin{array}{c}\text { Mar. 28, } \\
1950\end{array}$ \\
\hline
\end{tabular}

$a_{\text {Finished water. }}$

$b_{\text {Raw water. }}$ 


\section{BURLINGTON \\ (Population, 24, 560)}

Ownership: Municipal; also supplies suburban districts. Total population supplied, about 25, 000 .

Source: Stony Creek impounded. The raw water intake is located 3 miles north of the treatment plant.

Treatment: Coagulation with alum and lime, activated carbon, sedimentation, rapid sand filtration, break-point chlorination, and final adjustment of $\mathrm{pH}$ with lime.

Rated capacity of treatment plant: $5,500,000$ gpd.

Raw-water storage: Impounding reservoir, 500,000, 000 gal; reservoir at treatment plant, 1, 750, 000 gal.

Finished-water storage: Clear well, 465, 000 gal; 3 elevated tanks, 1, 500, 000, 100,000 , and 100, 000 gal.

The treatment plant is in the city.

\section{ANALYSES}

(Analyses, in parts per million, by U. S. Geological Survey)

\begin{tabular}{|c|c|c|c|c|c|}
\hline & $\begin{array}{l}\text { Raw } \\
\text { water }\end{array}$ & $\begin{array}{c}\text { Finished } \\
\text { water }\end{array}$ & & $\begin{array}{c}\text { Raw } \\
\text { water }\end{array}$ & $\begin{array}{l}\text { Finished } \\
\text { water }\end{array}$ \\
\hline $\begin{array}{l}\text { Silica }\left(\mathrm{SiO}_{2}\right) \\
\text { Iron }(\mathrm{Fe}) \ldots \ldots \ldots \ldots \\
\text { Manganese }(\ldots \ldots \ldots \ldots \\
(\mathrm{Mn})\end{array}$ & \multirow{2}{*}{$\begin{array}{c}16 \\
.16 \\
.00 \\
7.8 \\
3.6\end{array}$} & \multirow{2}{*}{$\begin{array}{c}15 \\
.04 \\
.00 \\
18 \\
3.5\end{array}$} & $\begin{array}{c}\text { Hardness as } \mathrm{CaCO}_{3}: \\
\text { Total ................. } \\
\text { Noncarbonate...... }\end{array}$ & $\begin{array}{r}34 \\
0\end{array}$ & $\begin{array}{l}59 \\
12\end{array}$ \\
\hline $\begin{array}{l}\text { Calcium (Ca) .......... } \\
\text { Magnesium (Mg)..... }\end{array}$ & & & \multirow{7}{*}{$\begin{array}{l}\text { Color ................... } \\
\text { pH .......................... } \\
\text { Specific conductance } \\
\text { (micromhos at } \\
25 \text { C.) ................ } \\
\text { Turbidity ............. } \\
\text { Temperature (F.)... } \\
\text { Date of collection... }\end{array}$} & \multirow{2}{*}{$\begin{array}{r}8 \\
6.8\end{array}$} & \multirow{2}{*}{$\begin{array}{r}3 \\
7.3\end{array}$} \\
\hline $\begin{array}{l}\text { Sodium (Na) .......... } \\
\text { Potassium (K) ....... }\end{array}$ & 5.1 & 4.9 & & & \\
\hline $\begin{array}{l}\text { Carbonate }\left(\mathrm{CO}_{3}\right) \ldots . . \\
\text { Bicarbonate }\left(\mathrm{HCO}_{3}\right)\end{array}$ & $\begin{array}{r}0 \\
45\end{array}$ & $\begin{array}{r}0 \\
58\end{array}$ & & 93.8 & 147 \\
\hline Sulfate $\left(\mathrm{SO}_{4}\right) \ldots \ldots \ldots$ & 3.4 & 16 & & -- & -- \\
\hline Chloride (Cl) .......... & 3.1 & 3.8 & & -- & \\
\hline $\begin{array}{l}\text { Fluoride }(\mathrm{F}) \\
\text { Nitrate }\left(\mathrm{NO}_{2}\right) \ldots \ldots \ldots \ldots\end{array}$ & $\begin{array}{r}.1 \\
.4\end{array}$ & $\begin{array}{r}.1 \\
.2\end{array}$ & & $\begin{array}{c}\text { Sept. 23, } \\
1950\end{array}$ & $\begin{array}{c}\text { Sept. 23, } \\
1950\end{array}$ \\
\hline Dissolved solids..... & 67 & 95 & & & \\
\hline
\end{tabular}

Regular determinations at treatment plant, 1950

\begin{tabular}{|c|c|c|c|c|c|c|c|c|c|c|c|c|}
\hline & \multicolumn{3}{|c|}{$\begin{array}{l}\text { Alkalinity } \\
\text { as CaCO } \\
\text { (ppm) }\end{array}$} & \multicolumn{3}{|c|}{ pH } & \multicolumn{3}{|c|}{$\begin{array}{l}\text { Hardness } \\
\text { as } \mathrm{CaCO} \\
\text { (ppm) }\end{array}$} & \multicolumn{3}{|c|}{ Turbidity } \\
\hline & Av & Max & Min & Av & Max & Min & Av & Max & Min & Av & Max & Min \\
\hline $\begin{array}{l}\text { Raw water......... } \\
\text { Finished water... }\end{array}$ & \begin{tabular}{|l|}
35 \\
40
\end{tabular} & $\begin{array}{l}57 \\
55\end{array}$ & \begin{tabular}{|l|}
16 \\
13 \\
\end{tabular} & $\begin{array}{l}7.0 \\
8.2\end{array}$ & \begin{tabular}{|l|}
7.4 \\
8.8
\end{tabular} & \begin{tabular}{l|}
6.6 \\
6.8
\end{tabular} & $\begin{array}{l}39 \\
58\end{array}$ & $\begin{array}{l}56 \\
78\end{array}$ & $\begin{array}{l}20 \\
38\end{array}$ & $\begin{array}{r}91 \\
0\end{array}$ & $\begin{array}{r}850 \\
0\end{array}$ & $\begin{array}{r}30 \\
0\end{array}$ \\
\hline
\end{tabular}


CHARLOTTE

(Population, 134, 042)

Ownership: Municipal; also supplies suburban districts. Total population supplied, about 167,000 .

Source: Catawba River. The intake is about 8 miles northwest of treatment plant.

Treatment: Aeration, coagulation with alum, carbon, primary chlorination, sedimentation, rapid sand filtration, final $\mathrm{pH}$ adjustment with hydrated lime, secondary chlorination, ammoniation, and fluoridation with sodium fluoride.

Rated capacity of treatment plant: $24,600,000$ gpd.

Raw-water storage: 2 reservoirs, estimated capacity 45, 000, 000 gal, each.

Finished-water storage: 3 clear wells, 2, 875,000, 2, 875, 000, and 5, 750, 000 gal;

4 elevated tanks, 1,000,000, 1,000,000,500,000, and 500,000 gal.

The treatment plant is about 2 miles northwest of center of city. The water is pumped from the Catawba River to raw-water storage; then flows by gravity through the treatment plant to clear water wells; then is pumped to distribution system and elevated storage tanks.

\section{ANALYSES}

(Analyses, in parts per million, by U. S. Geological Survey)

\begin{tabular}{|c|c|c|c|c|c|}
\hline & $\begin{array}{l}\text { Raw } \\
\text { water }\end{array}$ & $\begin{array}{c}\text { Finished } \\
\text { water }\end{array}$ & & $\begin{array}{l}\text { Raw } \\
\text { water }\end{array}$ & $\begin{array}{l}\text { Finished } \\
\text { water }\end{array}$ \\
\hline $\begin{array}{l}\text { Silica }\left(\mathrm{SiO}_{2}\right) \\
\text { Iron }(\mathrm{Fe}) \ldots \ldots \ldots \ldots \\
\text { Manganese }(\mathrm{Mn}) \ldots \ldots \ldots\end{array}$ & \multirow{2}{*}{$\begin{array}{r}12 \\
.03 \\
.00 \\
3.6 \\
1.2\end{array}$} & \multirow{2}{*}{$\begin{array}{r}11 \\
.02 \\
.00 \\
6.9 \\
1.2\end{array}$} & 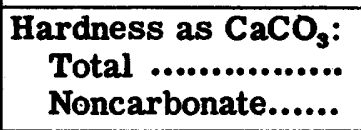 & $\begin{array}{r}14 \\
0\end{array}$ & $\begin{array}{r}22 \\
2\end{array}$ \\
\hline $\begin{array}{l}\text { Calcium }(\mathrm{Ca}) . . . . . . \\
\text { Magnesium }(\mathrm{Mg}) . . . .\end{array}$ & & & \multirow{6}{*}{$\begin{array}{c}\text { Color } \ldots . . . . . . . . . . . . . \\
\text { pH ........................ } \\
\text { Specific conductance } \\
\text { (micromhos at } \\
25 \text { C. ) ................ } \\
\text { Turbidity ............. } \\
\text { Temperature (F.)... } \\
\text { Date of collection... }\end{array}$} & 13 & 2 \\
\hline $\begin{array}{l}\text { Sodium (Na) .......... } \\
\text { Potassium (K) ....... }\end{array}$ & 3.6 & 4.9 & & 7.2 & 8.5 \\
\hline $\begin{array}{l}\text { Carbonate }\left(\mathrm{CO}_{3}\right) \ldots \ldots \\
\text { Bicarbonate }\left(\mathrm{HCO}_{3}\right) \\
\text { Sulfate }\left(\mathrm{SO}_{4}\right) \ldots \ldots . . . .\end{array}$ & $\begin{array}{l}0 \\
19 \\
2.7\end{array}$ & $\begin{array}{l}3 \\
18 \\
5,1\end{array}$ & & 40.5 & 75.4 \\
\hline Chloride (Cl) .......... & 2.2 & 3.2 & & - & -- \\
\hline 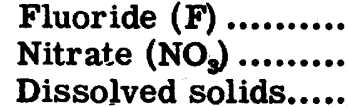 & .2 & $\begin{array}{r}1.2 \\
.2\end{array}$ & & $\begin{array}{l}\text { Mar. 2, } \\
1950\end{array}$ & $\underset{1950}{\operatorname{Mar} .3}$ \\
\hline & & 45 & & & \\
\hline
\end{tabular}

Regular determinations at treatment plant, 1950

\begin{tabular}{|c|c|c|c|c|c|c|c|c|c|c|c|c|}
\hline & \multicolumn{3}{|c|}{$\begin{array}{l}\text { Alkalinity } \\
\text { as } \mathrm{CaCO} \\
\text { (ppm) }\end{array}$} & \multicolumn{3}{|c|}{ pH } & \multicolumn{3}{|c|}{$\begin{array}{l}\text { Hardness } \\
\text { as } \mathrm{CaCO}_{3} \\
\text { (ppm) }\end{array}$} & \multicolumn{3}{|c|}{ Turbidity } \\
\hline & Av & Max & Min & Av & Max & Min & Av & Max & Min & $\mathbf{A v}$ & $\operatorname{Max}$ & Min \\
\hline $\begin{array}{l}\text { Raw water......... } \\
\text { Finished water... }\end{array}$ & $\begin{array}{l}15 \\
19\end{array}$ & \begin{tabular}{|l|}
17 \\
23
\end{tabular} & $\begin{array}{l}13 \\
16\end{array}$ & $\begin{array}{l}7.1 \\
8.6\end{array}$ & \begin{tabular}{|l|}
7.8 \\
8.8
\end{tabular} & $\begin{array}{l}6.9 \\
8.2\end{array}$ & $\begin{array}{l}12 \\
20\end{array}$ & $\begin{array}{l}16 \\
24\end{array}$ & $\begin{array}{r}9 \\
17\end{array}$ & $\begin{array}{l}8.1 \\
.06\end{array}$ & \begin{tabular}{|l|}
38 \\
.03 \\
\end{tabular} & $\begin{array}{l}2 \\
0\end{array}$ \\
\hline
\end{tabular}


CONCORD

(Population, 16, 486)

Ownership: Municipal; also supplies suburban districts. Total population supplied, about 20,000 .

Source: Cold Water Creek impounded in Lake Fisher. Lake Concord, fed by a number of springs, serves as auxiliary or emergency supply.

Treatment: Coagulation with alum, ammoniation, chlorination, activated carbon, sedimentation, rapid sand filtration, Calgon, lime, postchlorination, and final adjustment of $\mathrm{pH}$ with lime.

Rated capacity of treatment plant: 6,000,000 gpd.

Raw-water storage: Lake Fisher and Lake Concord 1, 100, 000,000, and 450, 000, 000 gal, respectively.

Finished-water storage: Clear well, 2,000, 000 gal; 2 elevated tanks, 500, 000 and $1,000,000$ gal; at old plant, 1,500, 000 gal.

The raw water intake is about 5 miles north of the treatment plant which is north of the city in Wil-Mar Park section.

\section{ANALYSES}

(Analyses, in parts per million, by U. S. Geological Survey)

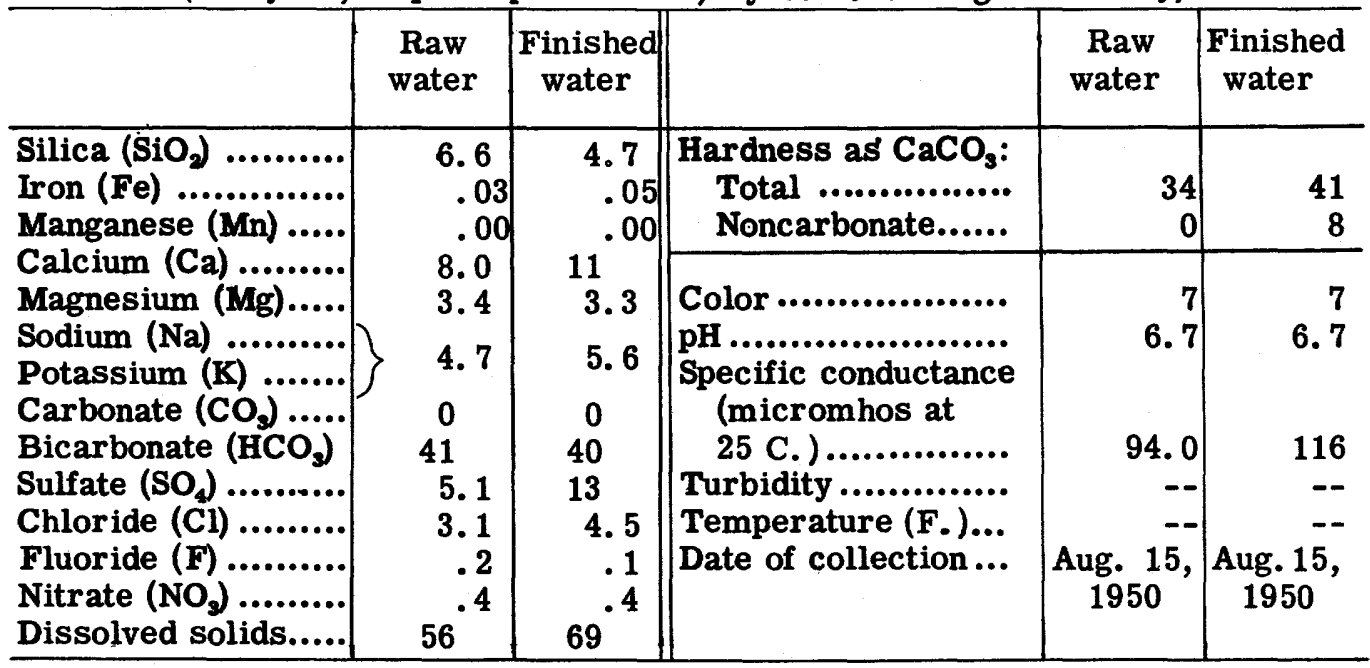

Regular determinations at treatment plant, 1950

\begin{tabular}{|c|c|c|c|c|c|c|c|c|c|c|c|c|}
\hline & \multicolumn{3}{|c|}{$\begin{array}{c}\text { Alkalinity } \\
\text { as } \mathrm{CaCO} \\
\text { (ppm) }\end{array}$} & \multicolumn{3}{|c|}{ pH } & \multicolumn{3}{|c|}{$\begin{array}{c}\text { Hardness } \\
\text { as } \mathrm{CaCO}_{3} \\
(\mathrm{ppm})\end{array}$} & \multicolumn{3}{|c|}{ Turbidity } \\
\hline & Av & Max & Min & Av & Max & $\operatorname{Min}$ & Av & $\operatorname{Max}$ & Min & Av & $\operatorname{Max}$ & Min \\
\hline $\begin{array}{l}\text { Raw water......... } \\
\text { Finished water... }\end{array}$ & $\begin{array}{l}32 \\
26\end{array}$ & $\begin{array}{l}36 \\
35\end{array}$ & $\begin{array}{l}18 \\
21\end{array}$ & $\begin{array}{l}7.2 \\
7.0\end{array}$ & \begin{tabular}{|l|}
7.5 \\
7.6
\end{tabular} & $\begin{array}{l}6.9 \\
6.8\end{array}$ & $\begin{array}{l}35 \\
43\end{array}$ & \begin{tabular}{|l|}
41 \\
47 \\
\end{tabular} & \begin{tabular}{|l|}
30 \\
38 \\
\end{tabular} & $\begin{array}{l}18 \\
--\end{array}$ & \begin{tabular}{l|l|}
40 \\
--
\end{tabular} & 10 \\
\hline
\end{tabular}




\section{DURHAM}

(Population 71, 311)

Ownership: Municipal; total population supplied, about 73, 500 .

Source: Flat River impounded in Lake Michie, about 15 miles northwest of the city.

Treatment: Slight aeration, breakpoint chlorination, coagulation with alum and lime, sedimentation, rapid sand filtration, and final adjustment of $\mathrm{pH}$ with lime. Activated carbon used occasionally for taste and odor control.

Rated capacity of treatment plant: $15,560,000$ gpd.

Raw-water storage: Impounding reservoir, 4, 500, 000, 000 gal.

Finished-water storage: 5 clear wells, 4,500,000 gal; 1 elevated tank, 1, 500,000 gal; 1 elevated reservoir, 3,000,000 gal.

The treatment plant is 3 miles northwest of the center of Durham. Treatment plant capacity can be increased satisfactorily to an additional 5, 530, 000 gpd.

\section{ANALYSES}

(Analyses, in parts per million, by U. S. Geological Survey)

\begin{tabular}{|c|c|c|c|c|c|}
\hline & $\begin{array}{l}\text { Raw } \\
\text { water }\end{array}$ & $\begin{array}{c}\text { Finished } \\
\text { water }\end{array}$ & & $\begin{array}{c}\text { Raw } \\
\text { water }\end{array}$ & $\begin{array}{c}\text { Finished } \\
\text { water }\end{array}$ \\
\hline $\begin{array}{l}\text { Silica }\left(\mathrm{SiO}_{2}\right) \ldots \ldots \ldots \ldots \\
\text { Iron }(\mathrm{Fe}) \ldots \ldots \ldots \ldots . . . \\
\text { Manganese }(\mathrm{Mn}) \ldots \ldots\end{array}$ & \multirow{2}{*}{$\begin{array}{r}13 \\
.04 \\
.00 \\
4.8 \\
2.0\end{array}$} & \multirow{2}{*}{$\begin{array}{r}14 \\
.05 \\
13 \\
.00 \\
2.0\end{array}$} & $\begin{array}{r}\text { Hardness as } \mathrm{CaCO}_{3} \text { : } \\
\text { Total .................. } \\
\text { Noncarbonate...... }\end{array}$ & $\begin{array}{r}20 \\
2\end{array}$ & $\begin{array}{l}41 \\
18\end{array}$ \\
\hline $\begin{array}{l}\text { Calcium (Ca) .......... } \\
\text { Magnesium (Mg)..... }\end{array}$ & & & Color ..................... & 13 & 4 \\
\hline $\begin{array}{l}\text { Sodium (Na) ........... } \\
\text { Potassium (K) ....... }\end{array}$ & 5.3 & 5.7 & pH ....................... & 7.2 & 8.7 \\
\hline $\begin{array}{l}\text { Carbonate }\left(\mathrm{CO}_{3}\right) \ldots . . . \\
\text { Bicarbonate }\left(\mathrm{HCO}_{3}\right)\end{array}$ & $\begin{array}{r}0 \\
22\end{array}$ & $\begin{array}{r}3 \\
21\end{array}$ & $\begin{array}{l}\text { (micromhos at } \\
25 \text { C. )..................... }\end{array}$ & 65.2 & 114 \\
\hline Sulfate $\left(\mathrm{SO}_{4}\right) \ldots \ldots \ldots$ & 5.7 & 20 & Turbidity ............... & -- & - \\
\hline $\begin{array}{l}\text { Chloride }(\mathrm{Cl}) \ldots \ldots \ldots \ldots \\
\text { Fluoride }(\mathrm{F}) \ldots \ldots \ldots \ldots\end{array}$ & $\begin{array}{r}5.1 \\
.1\end{array}$ & $\begin{array}{r}7.0 \\
.0\end{array}$ & $\begin{array}{l}\text { Temperature (F.)... } \\
\text { Date of collection... }\end{array}$ & Feb. 27, & Feb. 27, \\
\hline $\begin{array}{l}\text { Nitrate }\left(\mathrm{NO}_{3}\right) . . . . . . . \\
\text { Dissolved solids..... }\end{array}$ & $50^{.3}$ & $77^{.2}$ & & & 1950 \\
\hline
\end{tabular}

Regular determinations at treatment plant, 1950

\begin{tabular}{|c|c|c|c|c|c|c|c|c|c|c|c|c|}
\hline & \multicolumn{3}{|c|}{$\begin{array}{l}\text { Alkalinity } \\
\text { as CaCO } \\
\text { (ppm) }\end{array}$} & \multicolumn{3}{|c|}{ pH } & \multicolumn{3}{|c|}{$\begin{array}{c}\text { Hardness } \\
\text { as CaCO } \\
\text { (ppm) }\end{array}$} & \multicolumn{3}{|c|}{ Turbidity } \\
\hline & Av & $\operatorname{Max}$ & Min & Av & $\operatorname{Max}$ & Min & $\overline{A V}$ & Max & Min & Av & $\operatorname{Max}$ & Min \\
\hline $\begin{array}{l}\text { Raw water........ } \\
\text { Finished water.. }\end{array}$ & \begin{tabular}{|l|}
16 \\
27
\end{tabular} & $\begin{array}{l}20 \\
33\end{array}$ & \begin{tabular}{l|}
13 \\
23
\end{tabular} & $\begin{array}{l}6.9 \\
9.0\end{array}$ & \begin{tabular}{|l|}
7.1 \\
9.1
\end{tabular} & $\begin{array}{l}6.7 \\
9.0\end{array}$ & $\begin{array}{l}17 \\
44\end{array}$ & \begin{tabular}{|l|}
20 \\
51
\end{tabular} & $\begin{array}{l}14 \\
38\end{array}$ & $\begin{array}{r}19 \\
30\end{array}$ & \begin{tabular}{r|}
45 \\
.40
\end{tabular} & $\begin{array}{l}7.0 \\
.18\end{array}$ \\
\hline
\end{tabular}




\section{ELIZABETH CITY}

(Population, 12, 685)

Ownership: Municipal; also supplies suburban district. Total population supplied about 15,000 .

Source: Well fiela oî 200 wells, all 30 feet deep, 30 percent of supply; 3 wells, (1 to 3 ) each $80 \mathrm{ft}$ deep, 20 percent of supply. The yield of the shallow wells is reported to be from 2 to $5 \mathrm{gpm}$, and of the deep wells, 200, 200; and 90 gpm, respectively.

Treatment: Lime (softening), coagulation with alum, sedimentation, rapid sand filtration, and chlorination.

Rated capacity of treatment plant: 2,000,000 gpd.

Raw-water storage: 1 reservoir, 3,000,000 gal.

Finished-water storage: 1 clear well, 1, 000, 000 gal; 1 elevated tank, 500, 000 gal.

\section{ANALYSES}

(Analyses, in parts per million, by U. S. Geological Survey)

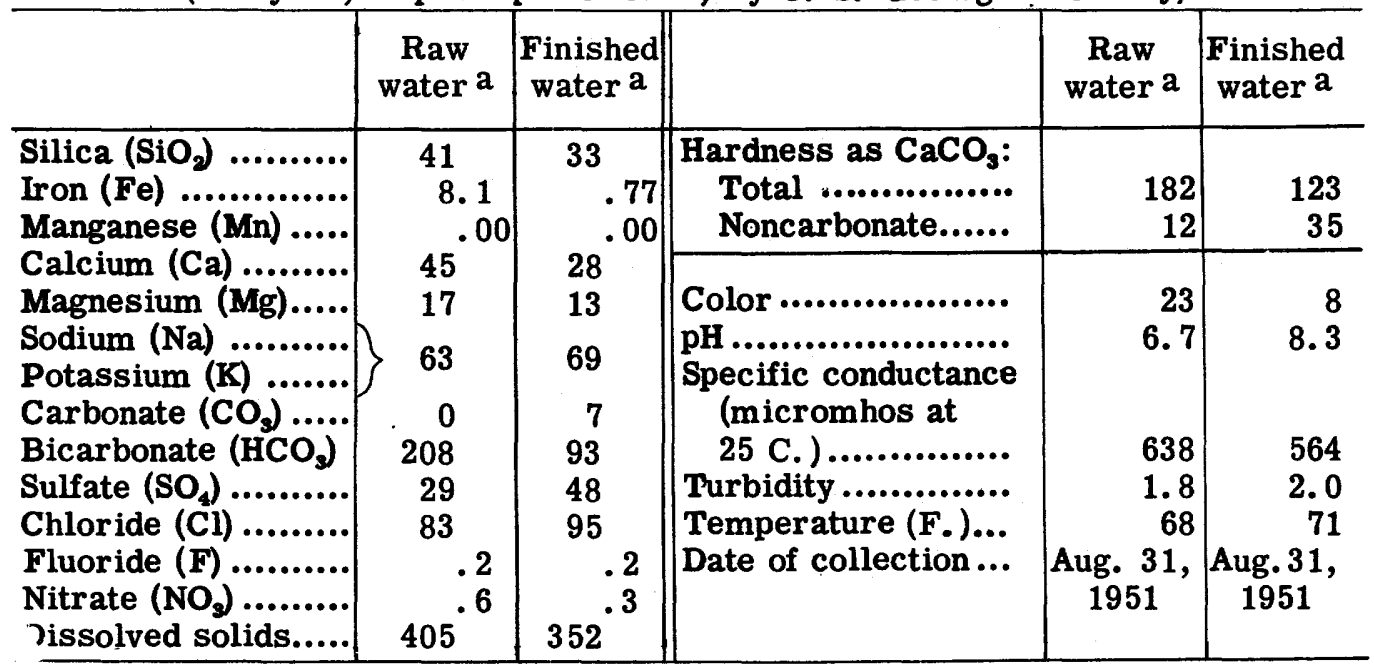

Regular determinations at treatment plant, 1950

\begin{tabular}{|c|c|c|c|c|c|c|c|c|c|c|c|c|}
\hline & \multicolumn{3}{|c|}{$\begin{array}{l}\text { Alkalinity } \\
\text { as CaCO } \\
\text { (ppm) }\end{array}$} & \multicolumn{3}{|c|}{ pH } & \multicolumn{3}{|c|}{$\begin{array}{c}\text { Hardness } \\
\text { as CaCO } \\
\text { (ppm) }\end{array}$} & \multicolumn{3}{|c|}{ Turbidity } \\
\hline & Av & $\operatorname{Max}$ & Min & Av & $\operatorname{Max}$ & Min & $\overrightarrow{A V}$ & Max & Min & Av & $\operatorname{Max}$ & Min \\
\hline $\begin{array}{l}\text { Raw water......... } \\
\text { Finished water... }\end{array}$ & $\begin{array}{r}115 \\
65\end{array}$ & $\begin{array}{r}180 \\
98\end{array}$ & $\begin{array}{l}98 \\
58\end{array}$ & $\begin{array}{l}7.0 \\
9.4\end{array}$ & $\begin{array}{l}7.2 \\
9.6\end{array}$ & \begin{tabular}{l|}
7.0 \\
9.0
\end{tabular} & $\begin{array}{l}140 \\
113\end{array}$ & \begin{tabular}{l|}
186 \\
130
\end{tabular} & $\begin{array}{r}110 \\
90\end{array}$ & -- & -- & -- \\
\hline
\end{tabular}

${ }^{a}$ Composite, mixed sample. 
FAYETTEVILLE

(Population, 34, 715)

Ownership: Municipal; also supplies other suburban districts. Total population supplied, about 50, 000 .

Source: Little Cross Creek impounded in Bonnie Doone, Kornbow, and Glenville Lakes. Intake on east bank of Glenville Lake about $200 \mathrm{ft}$ west of treatment plant.

Treatment: Aeration at times; primary lime, prechlorination, coagulation with alum, mechanical mixing, sedimentation, activated carbon at times, rapid sand filtration, postchlorination, Calgon, adjustment of $\mathrm{pH}$ with lime, ammoniation and final chlorination.

Rated capacity of treatment plant: $5,000,000 \mathrm{gpd}$.

Raw-water storage: 3 impounding reservoirs: Bonnie Doone, 90, 000, 000; Kornbow, 125, 000, 000; Glenville, 100,000, 000 gal.

Finished-water storage: 1 clear well, 1,500,000 gal; 2 elevated tanks, each $1,000,000$ gal.

The treatment plant is about 1 mile from center of city on Filter Plant Drive.

\section{ANALYSES}

(Analyses, in parts per million, by U. S. Geological Survey)

\begin{tabular}{|c|c|c|c|c|c|}
\hline & $\begin{array}{l}\text { Raw } \\
\text { water }\end{array}$ & $\begin{array}{c}\text { Finished } \\
\text { water }\end{array}$ & & $\begin{array}{c}\text { Raw } \\
\text { water }\end{array}$ & $\begin{array}{c}\text { Finished } \\
\text { water }\end{array}$ \\
\hline $\begin{array}{l}\text { Silica }\left(\mathrm{SiO}_{2}\right) \\
\text { Iron }(\mathrm{Fe}) \ldots \ldots \ldots \ldots \\
\text { Manganese }(\mathrm{Mn}) \ldots \ldots . . . . .\end{array}$ & $\begin{array}{l}3.4 \\
.08 \\
.00\end{array}$ & $\begin{array}{r}4.1 \\
.08 \\
.00\end{array}$ & $\begin{array}{r}\text { Hardness as } \mathrm{CaCO}_{3}: \\
\text { Total .................. } \\
\text { Noncarbonate...... }\end{array}$ & $\begin{array}{l}7 \\
2\end{array}$ & $\begin{array}{l}31 \\
16\end{array}$ \\
\hline $\begin{array}{l}\text { Calcium }(\mathrm{Ca}) . . . . . . \\
\text { Magnesium }(\mathrm{Mg}) . . . .\end{array}$ & $\begin{array}{r}1.6 \\
.8\end{array}$ & 11 & Color ..................... & 8 & 3 \\
\hline $\begin{array}{l}\text { Sodium (Na) ........... } \\
\text { Potassium (K) ...... }\end{array}$ & 2.3 & 2.2 & $\begin{array}{l}\text { pH ......................... } \\
\text { Specific conductance }\end{array}$ & 5.6 & 6.5 \\
\hline $\begin{array}{l}\text { Carbonate }\left(\mathrm{CO}_{3}\right) \ldots . . . \\
\text { Bicarbonate }\left(\mathrm{HCO}_{3}\right)\end{array}$ & $\begin{array}{l}0 \\
6\end{array}$ & $\begin{array}{r}0 \\
18\end{array}$ & $\begin{array}{l}\text { (micromhos at } \\
25 \text { C. }) \ldots \ldots \ldots \ldots \ldots\end{array}$ & -32.6 & 79.7 \\
\hline Sulfate $\left(\mathrm{SO}_{4}\right) \ldots \ldots \ldots$ & 2.2 & 10 & Turbidity ............... & - & -. \\
\hline Chloride (Cl) .......... & 3.2 & 7.5 & Temperature (F.)... & -- & -- \\
\hline Fluoride $(F) \ldots \ldots \ldots$ & .0 & .0 & Date of collection... & June 20, & June 20, \\
\hline $\begin{array}{l}\text { Nitrate }\left(\mathrm{NO}_{3}\right) \ldots . . . . . . \\
\text { Dissolved solids..... }\end{array}$ & $22^{.7}$ & $53^{.1}$ & & & \\
\hline
\end{tabular}

Regular determinations at treatment plant, 1950

\begin{tabular}{|c|c|c|c|c|c|c|c|c|c|c|c|c|}
\hline & \multicolumn{3}{|c|}{$\begin{array}{l}\text { Alkalinity } \\
\text { as } \mathrm{CaCO}_{3} \\
\text { (ppm) }\end{array}$} & \multicolumn{3}{|c|}{ pH } & \multicolumn{3}{|c|}{$\begin{array}{c}\text { Hardness } \\
\text { as } \mathrm{CaCO} \\
\text { (ppm) }\end{array}$} & \multicolumn{3}{|c|}{ Turbidity } \\
\hline & Av & $\operatorname{Max}$ & Min & Av & $\operatorname{Max}$ & Min & Av & $\operatorname{Max}$ & Min & Av & $\operatorname{Max}$ & Min \\
\hline $\begin{array}{l}\text { Raw water......... } \\
\text { Finished water... }\end{array}$ & $\begin{array}{r}4 \\
12\end{array}$ & $\begin{array}{r}5 \\
15\end{array}$ & $\begin{array}{l}3 \\
8\end{array}$ & $\begin{array}{l}6.0 \\
7.1\end{array}$ & $\begin{array}{l}6.1 \\
7.2\end{array}$ & $\begin{array}{l}5.9 \\
7.0\end{array}$ & $\begin{array}{r}6 \\
30\end{array}$ & $\begin{array}{r}6 \\
32\end{array}$ & $\begin{array}{r}6 \\
28\end{array}$ & $\begin{array}{r}20 \\
0\end{array}$ & $\begin{array}{r}40 \\
0\end{array}$ & $\begin{array}{r}10 \\
0\end{array}$ \\
\hline
\end{tabular}




\section{GASTONIA}

(Population, 23, 069)

Ownership: Municipal; also supplies Lowell, Dallas and other suburban districts. Total population supplied, about 48,200 .

Source: Long Creek impounded in Rankin Lake. The raw-water intake is about 3 miles north of city.

Treatment: Prechlorination, coagulation with alum, activated carbon (Nuchar), sedimentation, rapid sand filtration, postchlorination, Calgon, and lime.

Rated capacity of treatment plant: 4,800, 000 gpd.

Raw-water storage: Impounding reservoir, about 250,000, 000 gal.

Finished-water storage: 1 clear well 3,200, 000 gal; 2 elevated tanks 1, 000, 000 and 300,000 gal.

The treatment plant is almost in the center of the city.

\section{ANALYSES}

(Analyses, in parts per million, by U. S. Geological Survey)

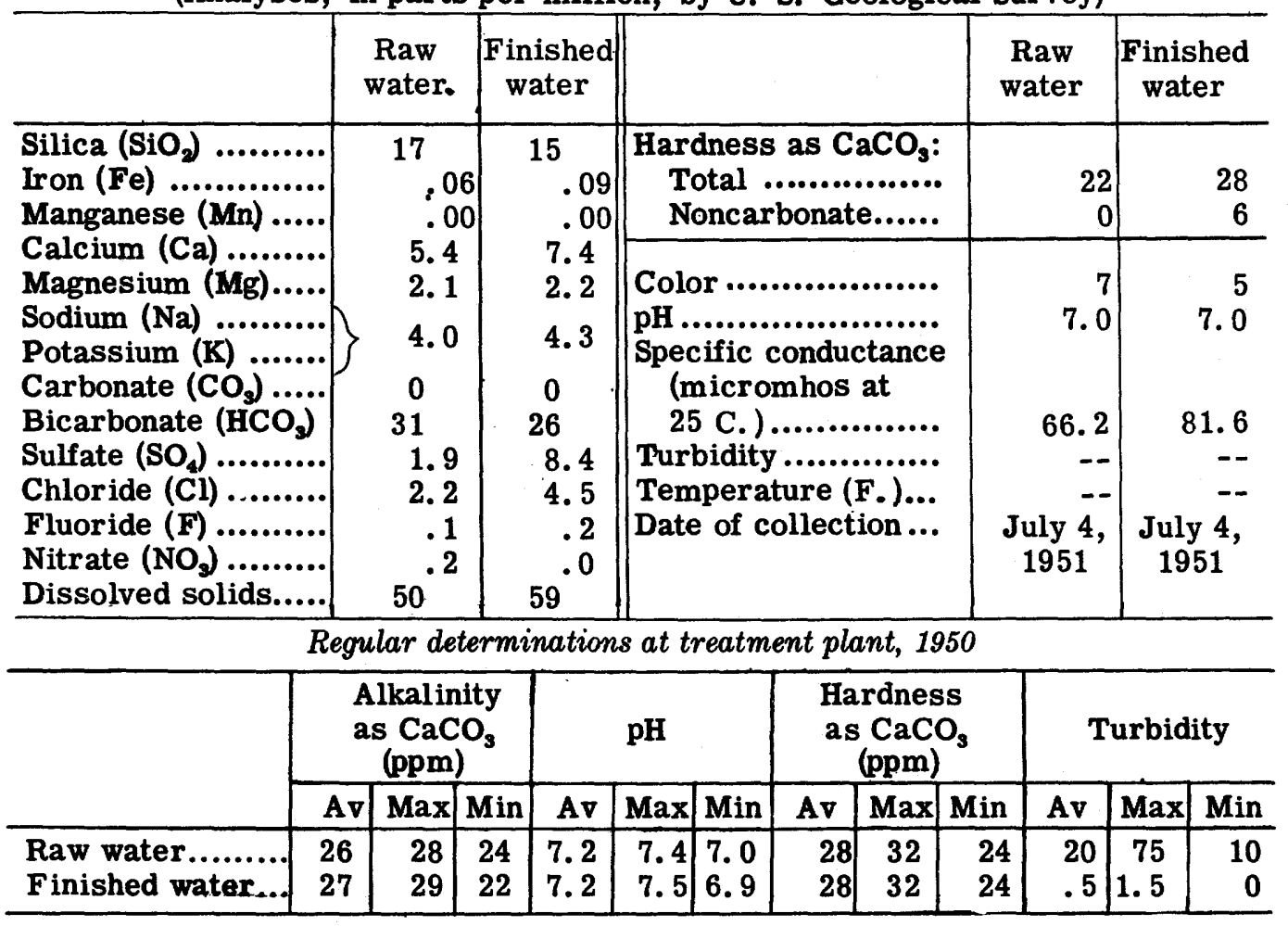




\section{GOLDSBORO \\ (Population, 21, 454)}

Ownership: Municipal; also supplies suburban districts. Total population supplied, about 25,000 .

Source: Little River. The intake is about $300 \mathrm{yd}$ northeast of the treatment plant. Treatment: Prechlorination, coagulation with alum and lime, sedimentation, rapid sand filtration, postchlorination, and final adjustment of $\mathrm{pH}$ with lime Rated capacity of treatmenc plant: 2, 250,000 gpd.

Raw-water storage: None.

Finished-water storage: Clear well, 500,000 gal; elevated taink, 1,000,000 gal.

The treatment plant is 2 miles northwest of Goldsboro on the south bank of Little River.

\section{ANALYSES}

(Analyses, in parts per million, by U. S. Geological Survey)

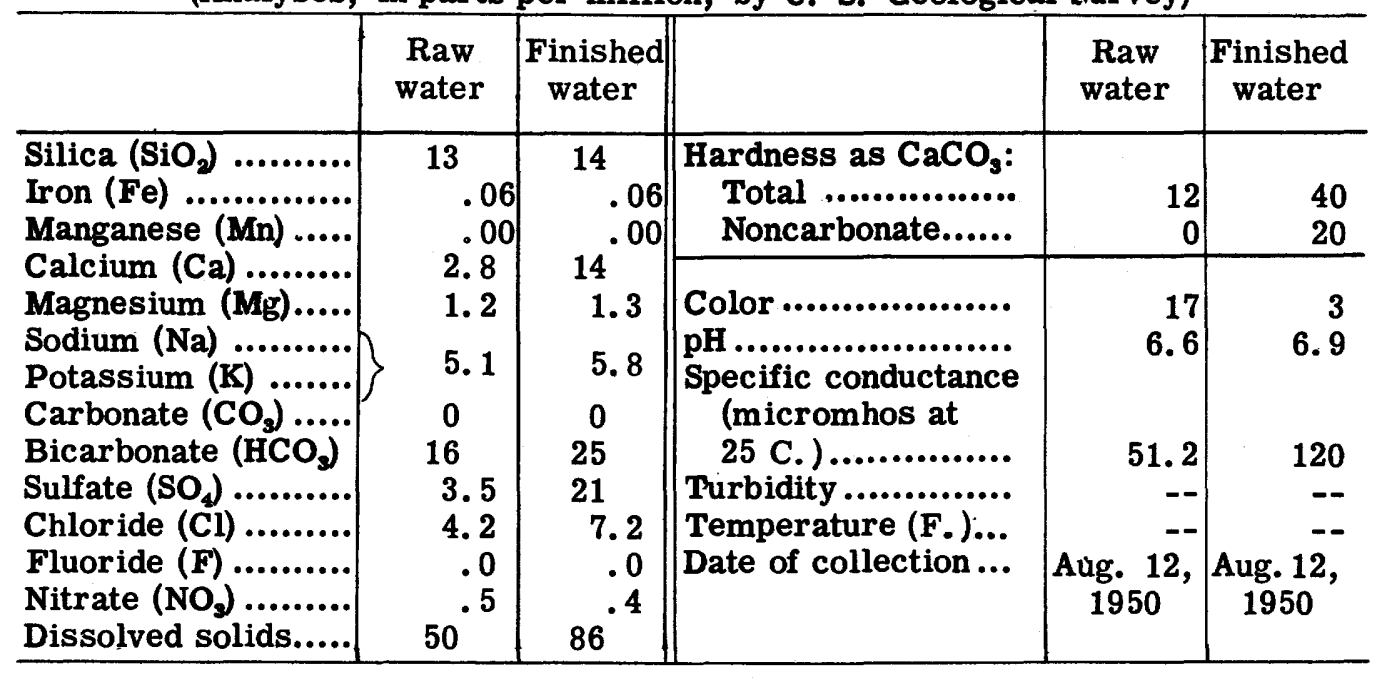




\section{GREENSBORO}

(Population, 74, 389)

Ownership: Municipal; also supplies suburban districts. Total population supplied, about 90,000 .

Source: Reedy Fork Creek (50 percent of supply), Horsepen Creek (30 percent of supply), Brush Creek (20 percent of supply) impounded in Lake Brandt. The intake is $6 \frac{1}{2}$ miles from the treatment plant.

Treatment: Prechlorination (at intermediate point on supply line to inhibit algae growth), coagulation with alum, sedimentation, rapid sand filtration, $\mathrm{pH}$ adjustment with lime, and postchlorination.

Rated capacity of treatment plant: $12,000,000 \mathrm{gpd}$.

Raw-water storage: Reservoir, 20, 000, 000 gal, Lake Brandt, 800, 000, 000 gal.

Finished-water storage: 2 clear wells, 3,000,000 and 18,000, 000 gal. 3 elevated tanks, 200, 000; 500,000, and 1,500,000 gal.

The treatment plant is in the north central section of the city.

\section{ANALYSES}

(Analyses, in parts per million, by U. S. Geological Survey)

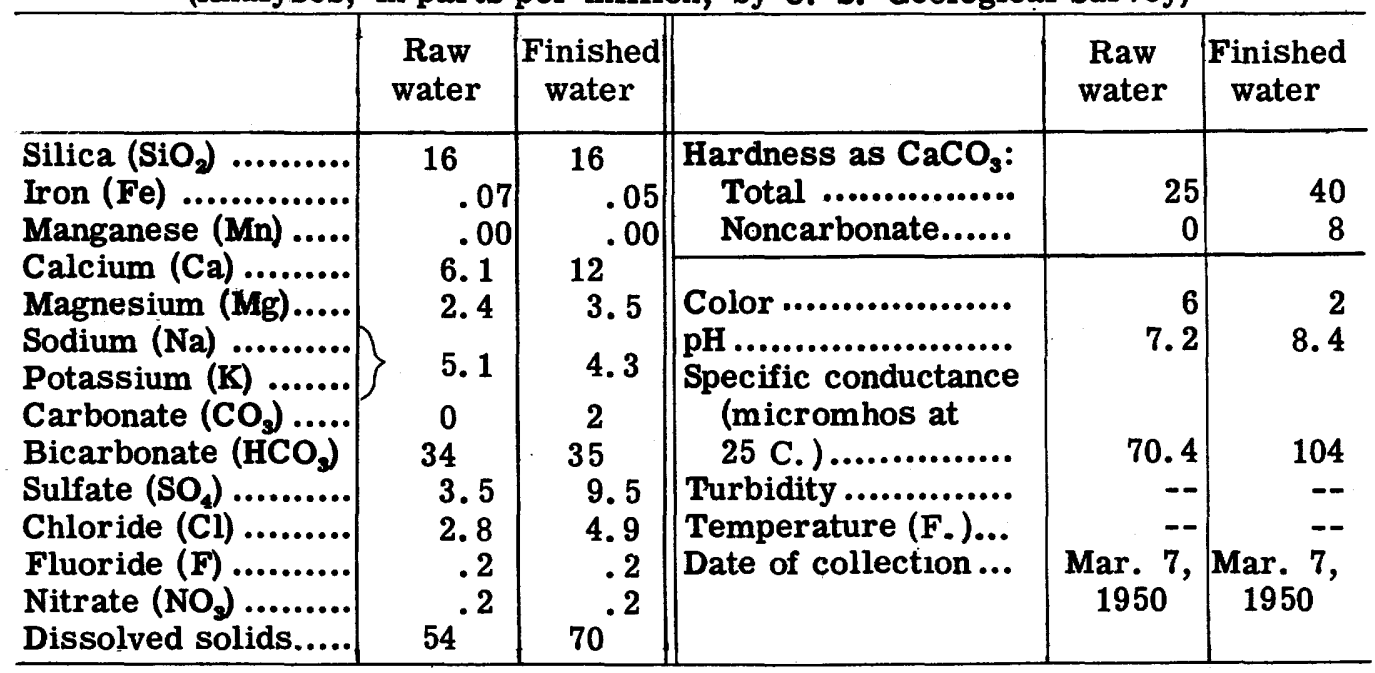

Regular determinations at treatment plant, 1950

\begin{tabular}{|c|c|c|c|c|c|c|c|c|c|c|c|c|}
\hline & \multicolumn{3}{|c|}{$\begin{array}{l}\text { Alkalinity } \\
\text { as CaCO } \\
\text { (ppm) }\end{array}$} & \multicolumn{3}{|c|}{$\mathrm{pH}$} & \multicolumn{3}{|c|}{$\begin{array}{l}\text { Hardness } \\
\text { as } \mathrm{CaCO}_{3} \\
\text { (ppm) }\end{array}$} & \multicolumn{3}{|c|}{ Turbidity } \\
\hline & Av & $\operatorname{Max}$ & Min & Av & $\operatorname{Max}$ & Min & Av & $\operatorname{Max}$ & Min & Av & $\operatorname{Max}$ & $\mathrm{Min}$ \\
\hline $\begin{array}{l}\text { Raw water.......... } \\
\text { Finished water... }\end{array}$ & $\begin{array}{l}30 \\
36\end{array}$ & $\begin{array}{l}36 \\
43\end{array}$ & $\begin{array}{l}26 \\
30\end{array}$ & $\begin{array}{l}7.0 \\
8.6\end{array}$ & $\begin{array}{l}7.5 \\
9.2\end{array}$ & $\begin{array}{l}6.7 \\
7.3\end{array}$ & $\begin{array}{l}24 \\
33\end{array}$ & \begin{tabular}{|l|}
46 \\
64 \\
\end{tabular} & $\begin{array}{l}17 \\
23\end{array}$ & $\begin{array}{r}62 \\
0\end{array}$ & $\begin{array}{r}160 \\
0\end{array}$ & $\begin{array}{r}30 \\
.0\end{array}$ \\
\hline
\end{tabular}


GREENVILLE

(Population, 16, 724)

Ownership: Municipal; also supplies suburban districts. Total population supplied, about 17,000 .

Source: Tar River.

Treatment: Coagulation with alum, ammoniation, chlorination, sedimentation, rapid sand filtration, and final adjustment of $\mathrm{pH}$ with lime.

Rated capacity of treatment plant: 3,000,000 gpd.

Raw - water storage: None.

Finished-water storage: 2 clear wells, 300,000 gal each; 3 elevated tanks, 300,000 gal each.

The treatment plant is in the city near Tar River.

\section{ANALYSES}

(Analyses, in parts per million, by U. S. Geological Survey)

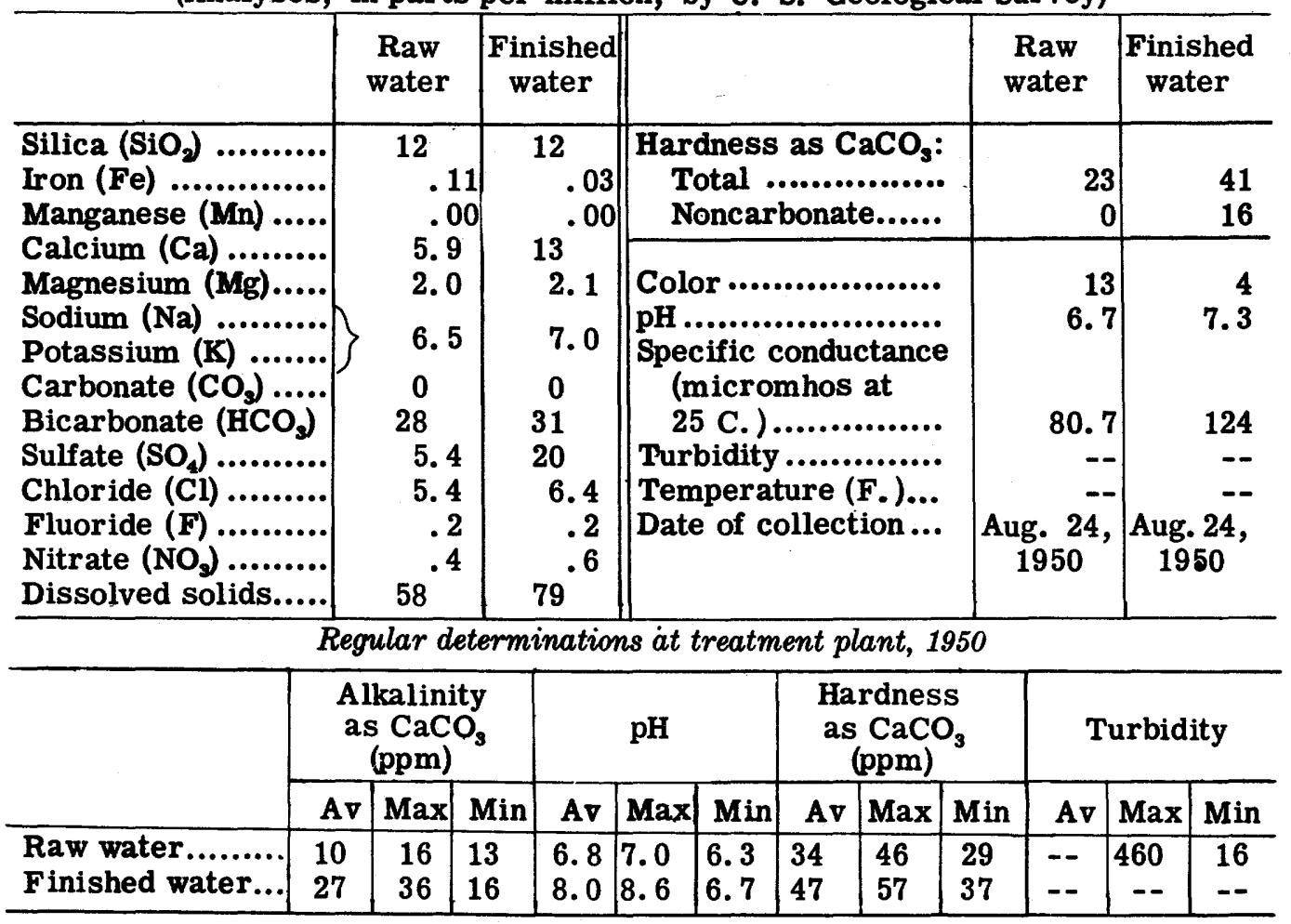




\section{HENDERSON \\ (Population, 10, 996)}

Ownership: Municipal; also supplies suburban districts. Total population supplied, about 14,500 .

Source: Fox Pond impounding reservoir for East Sandy Creek. Intake is about $1 \frac{1}{2}$ miles south of the city. Rowland Lake, auxiliary or emergency supply.

Treatment: Coagulation with alum and lime, sedimentation, rapid sand filtration, chlorination, Calgon, and adjustment of $\mathrm{pH}$ with soda ash.

Rated capacity of treatment plant: 2, 000, 000 gpd.

Raw-water storage: Impounding reservoir, 40, 000, 000 gal (estimated).

Finished-water storage: 1 clear well, 750, 000 gal; 1 standpipe, 172, 000 gal.

\section{ANALYSES}

(Analyses, in parts per million, by U. S. Geological Survey)

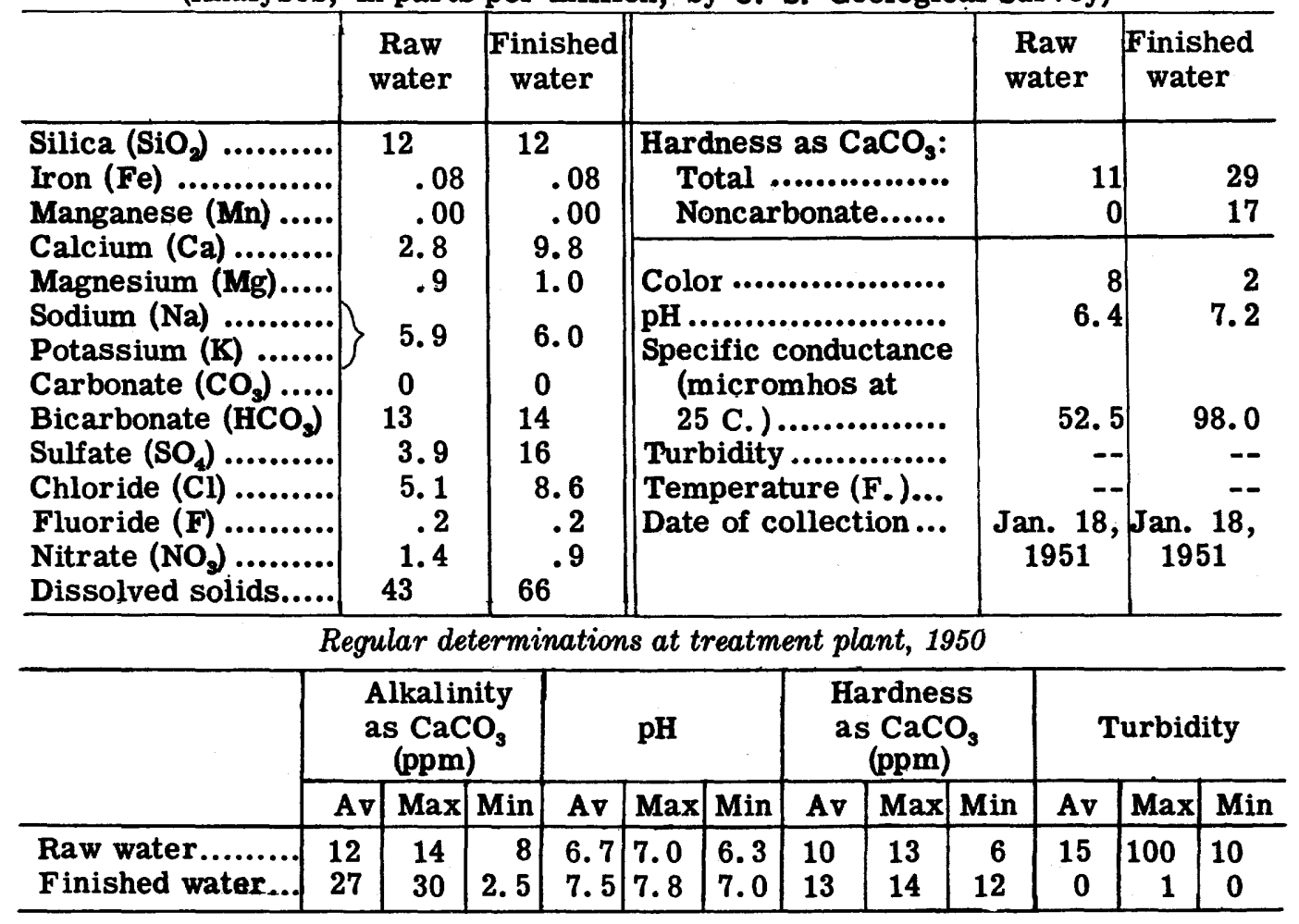




\section{HICKORY}

(Population, 14, 755)

Ownership: Municipal; also supplies Longview, Brookford, Windy City area, and suburban districts. Total population supplied, about 20,000 .

Source: Catawba River impounded in Lake Hickory. The intake is $1,500 \mathrm{ft}$ west of the treatment plant.

Treatment: Coagulation with alum, chlorination, sedimentation, rapid sand filtration, and final adjustment of $\mathrm{pH}$ to about 8.5 by addition of lime.

Rated capacity of treatment plant: $4,000,000$ gpd.

Kaw-water storage: Lake Hickory, 32, 440,000, 000 gal.

Finished-water storage: 1 clear well, 500,000 gal; 1 elevated tank, 1,000,000 gal; 2 standpipes, 250,000 gal each.

The treatment plant is 2 miles northwest of center of the city.

\section{ANALYSES}

(Analyses, in parts per million, by U. S. Geological Survey)

\begin{tabular}{|c|c|c|c|c|c|}
\hline & $\begin{array}{c}\text { Raw } \\
\text { water }\end{array}$ & $\begin{array}{c}\text { Finished } \\
\text { water }\end{array}$ & & $\begin{array}{c}\text { Raw } \\
\text { water }\end{array}$ & $\begin{array}{l}\text { Finished } \\
\text { water }\end{array}$ \\
\hline $\begin{array}{l}\text { Silica }\left(\mathrm{SiO}_{2}\right) \\
\text { Iron }(\mathrm{Fe}) \ldots \ldots \ldots \ldots \\
\text { Manganese }(\mathrm{Mn}) \ldots \ldots . . . .\end{array}$ & $\begin{array}{r}11 \\
.07 \\
.12\end{array}$ & $\begin{array}{l}9.8 \\
.07 \\
.08\end{array}$ & 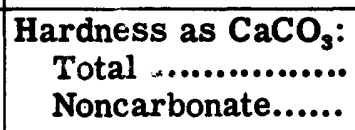 & $\begin{array}{r}14 \\
0\end{array}$ & $\begin{array}{r}26 \\
3\end{array}$ \\
\hline $\begin{array}{l}\text { Calcium (Ca) .......... } \\
\text { Magnesium (Mg)..... }\end{array}$ & $\begin{array}{l}3.5 \\
1.4\end{array}$ & $\begin{array}{l}8.2 \\
1.4\end{array}$ & Color ...................... & 3 & 3 \\
\hline Sodium (Na) ........... & 3.6 & 3.3 & pH ......................... & 6.4 & 6.9 \\
\hline $\begin{array}{l}\text { Carbonate }\left(\mathrm{CO}_{3}\right) \ldots . . . \\
\text { Bicarbonate }\left(\mathrm{HCO}_{3}\right)\end{array}$ & $\begin{array}{r}0 \\
20\end{array}$ & $\begin{array}{r}0 \\
28\end{array}$ & 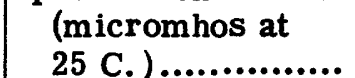 & 44.9 & 70.2 \\
\hline Sulfate $\left(\mathrm{SO}_{4}\right) \ldots . . . . .$. & 1.9 & 5.5 & Turbidity ............... & -- & -- \\
\hline Chloride (Cl) . & 2.2 & 3.0 & Temperature (F.)... & -- & -- \\
\hline $\begin{array}{l}\text { Fluoride (F) ........... } \\
\text { Nitrate }\left(\mathrm{NO}_{3}\right) \ldots \ldots . . . \\
\text { Dissolved solids..... }\end{array}$ & $34^{.6}$ & $48^{.3}$ & Date of collection... & $\begin{array}{c}\text { Aug. 15, } \\
1950\end{array}$ & $\begin{array}{c}\text { Aug. 15, } \\
1950\end{array}$ \\
\hline
\end{tabular}

Regular determinations at treatment plant, 1950

\begin{tabular}{|c|c|c|c|c|c|c|c|c|c|c|c|c|}
\hline & \multicolumn{3}{|c|}{$\begin{array}{l}\text { Alkalinity } \\
\text { as } \mathrm{CaCO} \\
\text { (ppm) }\end{array}$} & \multicolumn{3}{|c|}{$\mathrm{pH}$. } & \multicolumn{3}{|c|}{$\begin{array}{c}\text { Hardness } \\
\text { as } \mathrm{CaCO} \\
(\mathrm{ppm})\end{array}$} & \multicolumn{3}{|c|}{ Turbidity } \\
\hline & Av & $\operatorname{Max}$ & Min & Av & Max & Min & Av & Max & Min & Av & $\operatorname{Max}$ & Min \\
\hline Raw water......... & 10 & 12 & 9 & 6.7 & 6.8 & 6.6 & 12 & 15 & 11 & 30 & 60 & 25 \\
\hline Finished water... & 27 & 30 & 24 & 8.6 & 8.8 & 8.3 & 23 & 29 & 21 & 0 & 0 & 0 \\
\hline
\end{tabular}


HIGH POINT

(Population, 39, 973)

Ownership: Municipal; also supplies suburban districts. Total population supplied, about 42,500 .

Source: Deep River impounded. The intake on Deep River is $4 \frac{1}{2}$ miles northeast of treatment plant, which is 1 mile east of the city.

Treatment: Breakpoint chlorination, coagulation with alum, sedimentation, rapid sand filtration, postchlorination, ammoniation, and final adjustment of pH with lime.

Rated capacity of treatment plant: 7,500, 000 gpd.

Raw-water storage: Impounding reservoir, 1, 500, 000, 000 gal.

Finished-water storage: 1 clear well, 3,000,000 gal; 1 elevated tank, 1, 000, 000 gal.

\section{ANALYSES}

(Analyses, in parts per million, by U. S. Geological Survey)

\begin{tabular}{|c|c|c|c|c|c|}
\hline & $\begin{array}{l}\text { Raw } \\
\text { water }\end{array}$ & $\begin{array}{c}\text { Finished } \\
\text { water }\end{array}$ & & $\begin{array}{l}\text { Raw } \\
\text { water }\end{array}$ & $\begin{array}{l}\text { Finished } \\
\text { water }\end{array}$ \\
\hline $\begin{array}{l}\text { Silica }\left(\mathrm{SiO}_{2}\right) \\
\text { Iron }(\mathrm{Fe}) \ldots \ldots \ldots \ldots \\
\text { Manganese }(\mathrm{Mn}) \ldots \ldots .\end{array}$ & \multirow{2}{*}{$\begin{array}{r}19 \\
.13 \\
.00 \\
6.1 \\
2.8\end{array}$} & \multirow{2}{*}{\begin{tabular}{|c||}
18 \\
.04 \\
.00 \\
12 \\
3.0
\end{tabular}} & $\begin{array}{r}\text { Hardness as } \mathrm{CaCO}_{3}: \\
\text { Total .................. } \\
\text { Noncarbonate...... }\end{array}$ & $\begin{array}{r}27 \\
0\end{array}$ & $\begin{array}{l}42 \\
10\end{array}$ \\
\hline $\begin{array}{l}\text { Calcium }(\mathrm{Ca}) . . . . . . \\
\text { Magnesium }(\mathrm{Mg}) . . .\end{array}$ & & & Color .................... & $\mathbf{5}$ & \\
\hline & 7.3 & 5.6 & & 7.1 & 7.3 \\
\hline $\begin{array}{l}\text { Carbonate }\left(\mathrm{CO}_{3}\right) \ldots \\
\text { Bicarbonate }\left(\mathrm{HCO}_{3}\right)\end{array}$ & & & & 82.3 & 115 \\
\hline Sulfate $\left(\mathrm{SO}_{4}\right) \ldots . . . . .$. & 5.6 & 15 & Turbidity .............. & -- & - \\
\hline Chloride (Cl) .......... & 3.6 & 4.8 & Temperature (F.)... & - & - \\
\hline $\begin{array}{l}\text { Fluoride }(\mathrm{F}) \\
\text { Nitrate }\left(\mathrm{NO}_{2}\right) \ldots \ldots \ldots\end{array}$ & $\begin{array}{l}.1 \\
.3\end{array}$ & $\begin{array}{r}.1 \\
.0\end{array}$ & Date of collection... & $\underset{1950}{\text { Mar. 17, }}$ & $\underset{1950}{\text { Mar. } 17,}$ \\
\hline Dissolved solids..... & 64 & 79 & & & \\
\hline
\end{tabular}

Regular determinations at treatment plant, 1950

\begin{tabular}{|c|c|c|c|c|c|c|c|c|c|c|c|c|}
\hline \multirow[t]{2}{*}{. } & \multicolumn{3}{|c|}{$\begin{array}{c}\text { Alkalinity } \\
\text { as } \mathrm{CaCO} \\
\text { (ppm) }\end{array}$} & \multicolumn{3}{|c|}{$\mathrm{pH}$} & \multicolumn{3}{|c|}{$\begin{array}{l}\text { Hardness } \\
\text { as } \mathrm{CaCO}_{3} \\
\text { (ppm) }\end{array}$} & \multicolumn{3}{|c|}{ Turbidity } \\
\hline & Av & $\operatorname{Max}$ & Min & Av & $\operatorname{Max}$ & Min & Av & Max & Min & Av & $\operatorname{Max}$ & Min \\
\hline $\begin{array}{l}\text { Raw water.......... } \\
\text { Finished water... }\end{array}$ & \begin{tabular}{l|}
29 \\
34
\end{tabular} & \begin{tabular}{|l|}
38 \\
48 \\
\end{tabular} & $\begin{array}{l}22 \\
26\end{array}$ & $\begin{array}{l}6.9 \\
7.5\end{array}$ & \begin{tabular}{|l|}
7.3 \\
8.8
\end{tabular} & \begin{tabular}{|l|}
6.7 \\
6.8
\end{tabular} & $\begin{array}{l}30 \\
48\end{array}$ & $\begin{array}{l}32 \\
52\end{array}$ & \begin{tabular}{|l|}
28 \\
42 \\
\end{tabular} & $\begin{array}{l}88 \\
--\end{array}$ & \begin{tabular}{|c|}
350 \\
---
\end{tabular} & \begin{tabular}{|l|}
40 \\
--
\end{tabular} \\
\hline
\end{tabular}




\section{KANNAPOLIS}

(Population, 28, 448)

Ownership: Cannon Mills, also supplies suburban districts. Total population supplied, about 33,000 .

Source: Buffalo Creek impounded for the regular supply. Coddle Creek serves as an auxiliary supply. The intake at the dam is about $1 \frac{1}{2}$ miles west of Kannapolis.

Treatment: Domestic: Prechlorination, coagulation with alum, sedimentation, carbon, rapid sand filtration, ammoniation, postchlorination, and soda ash. Industrial: Prechlorination, coagulation with alum, sedimentation, carbon, rapid sand filtration, and soda ash.

Rated capacity of treatment plant: $7,500,000 \mathrm{gpd}$.

Raw-water storage: Impounding reservoir, 1,250, 000, 000 gal.

Finished-water storage: Drinking water system: 2 elevated tanks, 250, 000 gal each; 2 clear wells, 100,000 and 75,000 gal. Industrial and sprinkler system: 1 open reservoir, 9, 800, 000; 2 standpipes, 250, 000 gal each; 4 elevated tanks, 250,000,100,000, 100,000, and 75, 000 gal.

\section{ANALYSES}

(Analyses, in parts per million, by U. S. Geological Survey)

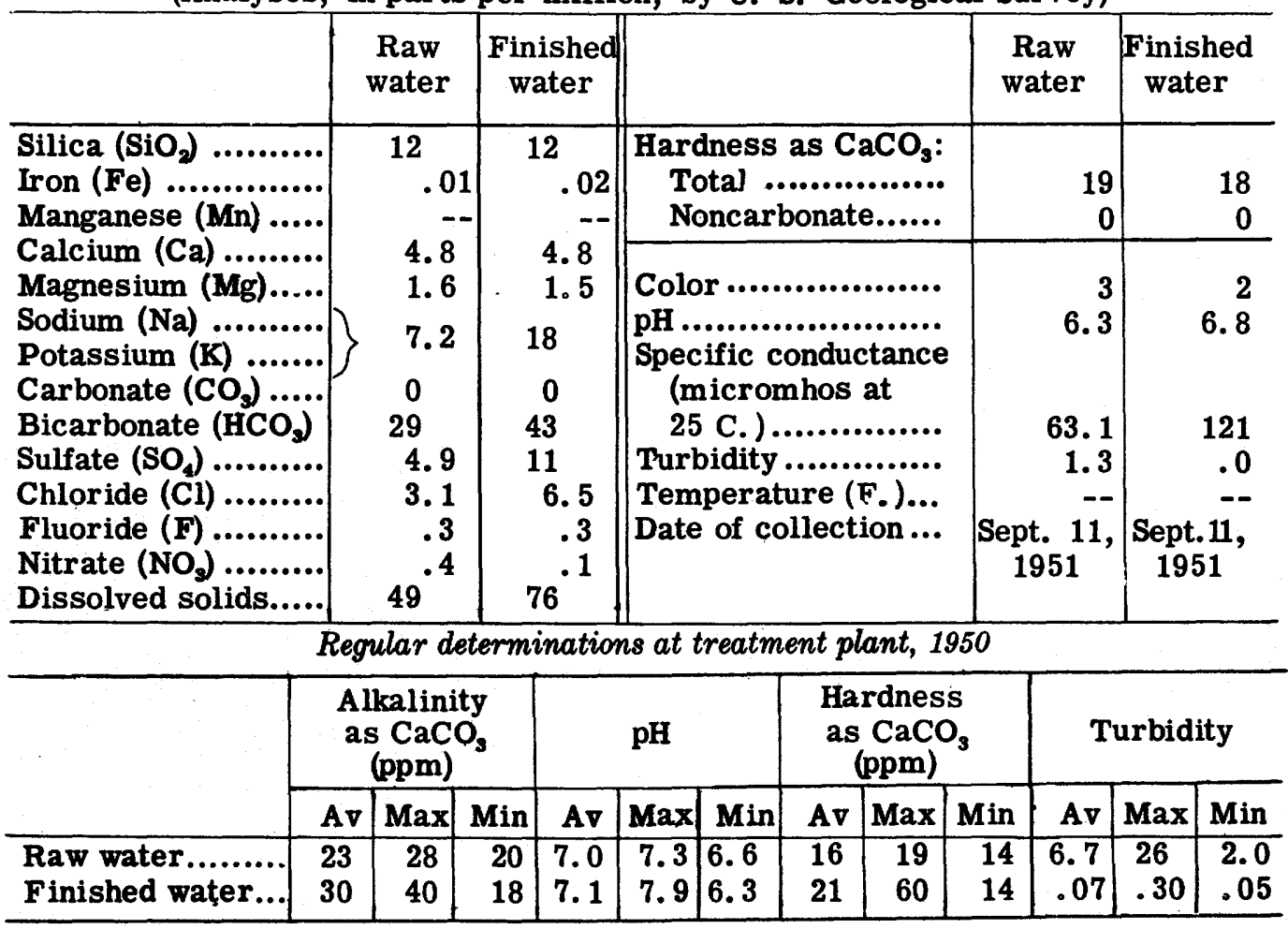




\section{KINSTON}

(Population, 18, 336)

Ownership: Municipal; also supplies suburban districts. Total population supplied, 22,000 .

Source: 4 wells ( 1 to 4$) 361,369,365$, and $552 \mathrm{ft}$ deep; 3 artesian wells (Hillcrest group) 350 to $390 \mathrm{ft}$ deep. The yield of the four wells is reported to be 900 , $690,460,900 \mathrm{gpm}$. The flow of the artesian group is $150 \mathrm{gpm}$.

Treatment: The water from wells 1 to 4 is chlorinated before entering the distribution system. Water from the Hillcrest wells flows into a reservoir and is then pumped into the distribution system.

Finished-water storage: 2 reservoirs, 1,000,000 and 250,000 gal; 1 elevated tank, 500, 000 gal.

\section{ANALYSES}

(Analyses, in parts per million, by U. S. Geological Survey)

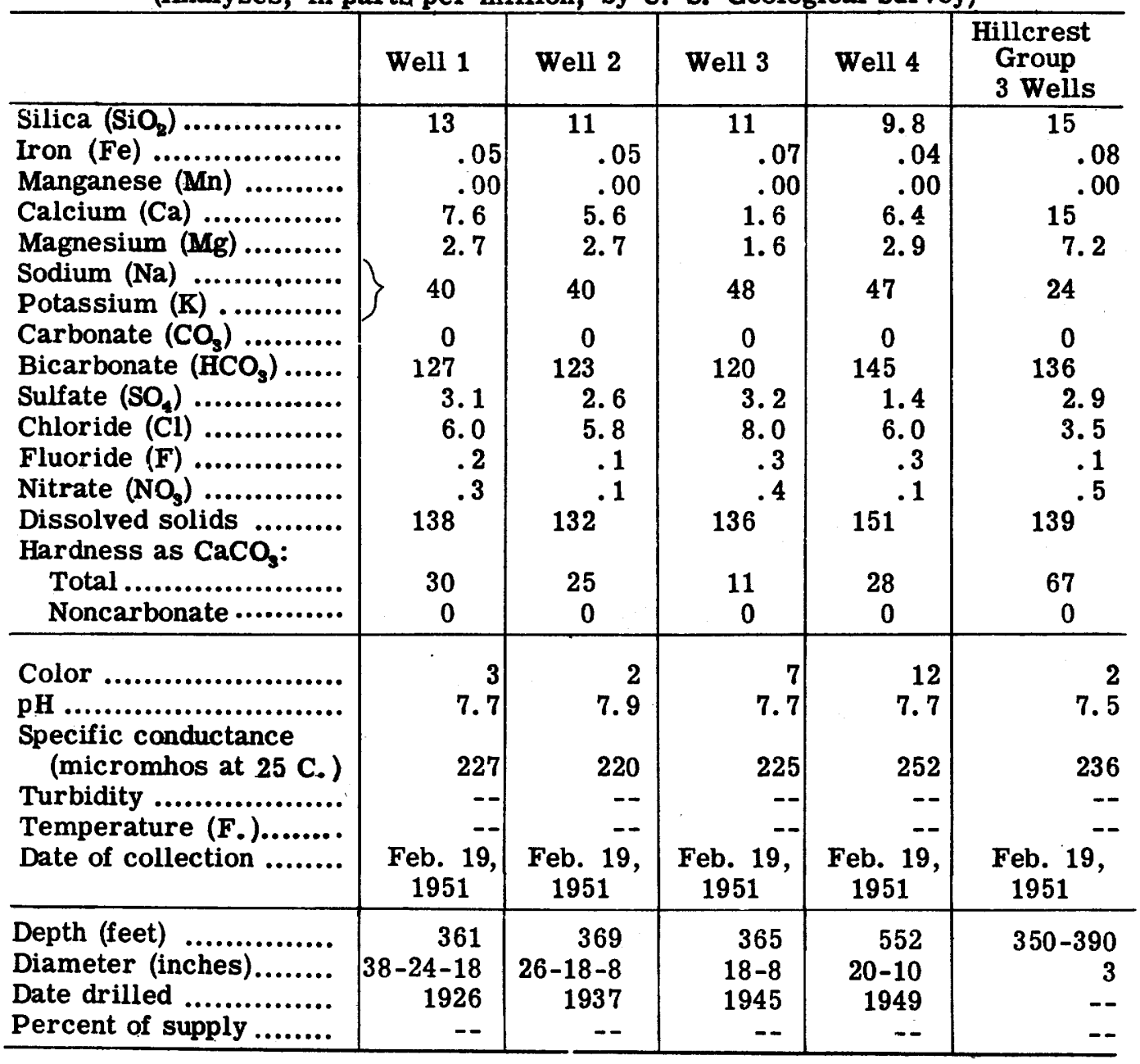




\section{LEXINGTON}

(Population, 13, 571)

Ownership: Municipal; also supplies suburban districts. . Total population supplied, about 16,062 .

Source: The Abbotts Creek intake is about 4 miles northeast of the city. Leonard Creek impounded in Leonard Lake is used as an auxiliary or emergency supply.

Treatment: Prechlorination, alum, air-mix, flocculation, sedimentation, rapid sand filtration, postchlorination (if required), final adjustment of $\mathrm{pH}$ with lime.

Rated capacity of treatment plant: $3,500,000 \mathrm{gpd}$.

Raw-water storage: Leonard Lake, 365, 000, 000 gal.

Finished-water storage: At plant, 1,000, 000 gal; 1 elevated tank, 1, 000, 000 gal; 1 standpipe, 350, 000 gal.

The treatment plant is located 4.1 miles northeast of Lexington on the Greensboro Road.

\section{ANALYSES}

(Analyses, in parts per million, oy U. S. Geological Survey)

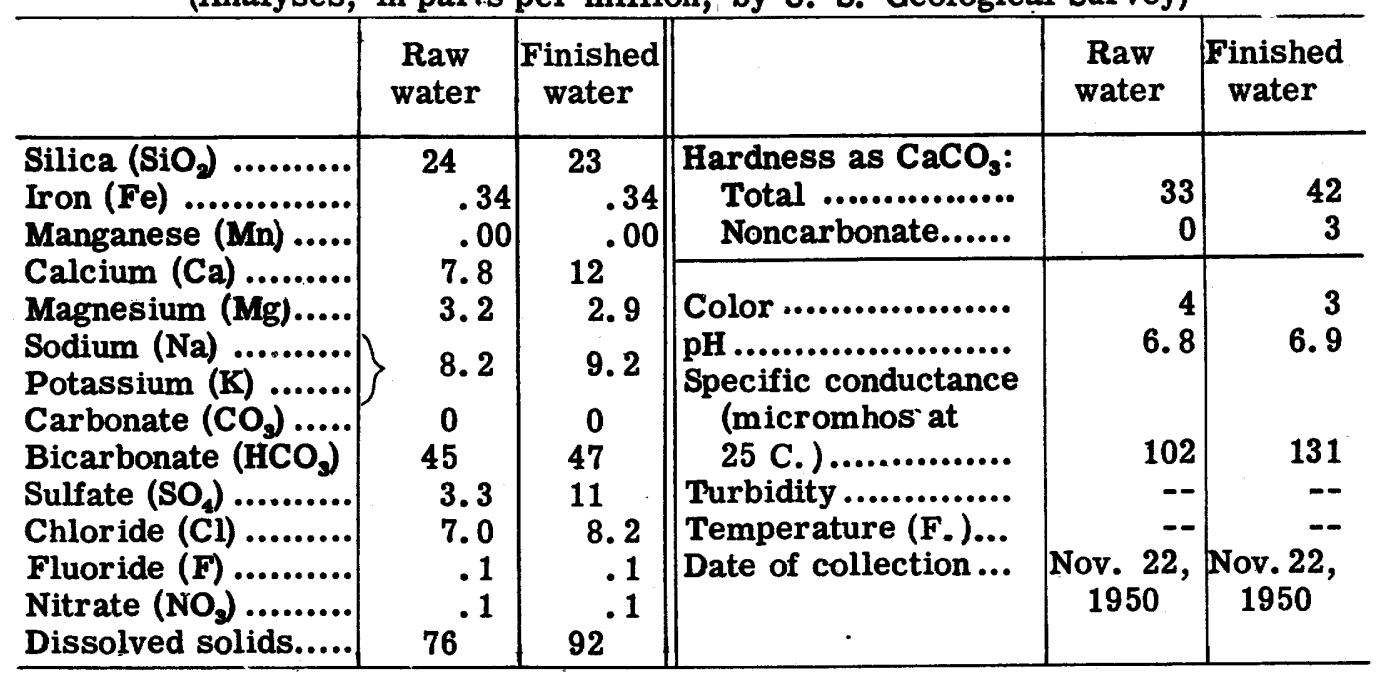

Regular determinations at treatment plant, 1950

\begin{tabular}{|c|c|c|c|c|c|c|c|c|c|c|c|c|}
\hline & \multicolumn{3}{|c|}{$\begin{array}{c}\text { Alkalinity } \\
\text { as } \mathrm{CaCO} \\
\text { (ppm) }\end{array}$} & \multicolumn{3}{|c|}{$\mathrm{pH}$} & \multicolumn{3}{|c|}{$\begin{array}{c}\text { Hardness } \\
\text { as } \mathrm{CaCO} \\
\text { (ppm) }\end{array}$} & \multicolumn{3}{|c|}{ Turbidity } \\
\hline & $\mathrm{Av}$ & $\operatorname{Max}$ & Min & Av & $\operatorname{Max}$ & Min & Av & Max & Min & Av & Max & Min \\
\hline $\begin{array}{l}\text { Raw water.......... } \\
\text { Finished water.. }\end{array}$ & \begin{tabular}{l|}
28 \\
36
\end{tabular} & \begin{tabular}{l|}
32 \\
42
\end{tabular} & $\begin{array}{l}25 \\
24\end{array}$ & $\begin{array}{l}7.1 \\
8.4\end{array}$ & $\begin{array}{l}7.5 \\
8.6\end{array}$ & $\begin{array}{l}6.8 \\
8.4\end{array}$ & $\begin{array}{l}28 \\
36\end{array}$ & $\begin{array}{l}30 \\
40\end{array}$ & \begin{tabular}{|l|}
25 \\
32
\end{tabular} & $\begin{array}{l}50 \\
--\end{array}$ & $\begin{array}{r}3000 \\
-- \\
\end{array}$ & $\begin{array}{l}18 \\
-- \\
\end{array}$ \\
\hline
\end{tabular}




\section{MONROE}

(Population, 10, 140)

Ownership: Municipal; also supplies Wingate, Marshville and other suburban districts. Total population supplied, about 12,600.

Source: Richardson Creek impounded in Lake Lee. The intake at Lake is 2 miles east of treatment plant, which is in the city.

Treatment: Prechlorination, coagulation with alum, lime, activated carbon (Nuchar), sedimentation, rapid sand filtration, and postchlorination.

Rated capacity of treatment plant: 2, 000, 000 gpd.

Raw-water storage: 450,000,000 gal.

Finished-water storage: 2 clear wells, 420,000 and 540,000 gal; 4 elevated tanks, $165,000,500,000,135,000$ and 50,000 gal.

\section{ANALYSES}

(Analyses, in parts per million, by U. S. Geological Survey)

\begin{tabular}{|c|c|c|c|c|c|}
\hline & $\begin{array}{c}\text { Raw } \\
\text { water }\end{array}$ & $\begin{array}{c}\text { Finished } \\
\text { water }\end{array}$ & & $\begin{array}{c}\text { Raw } \\
\text { water }\end{array}$ & $\begin{array}{c}\text { Finished } \\
\text { water }\end{array}$ \\
\hline \multirow{7}{*}{$\begin{array}{l}\text { Silica }\left(\mathrm{SiO}_{2}\right) \\
\text { Iron }(\mathrm{Fe}) \ldots \ldots \ldots \\
\text { Manganese }(\mathrm{Mn}) \ldots \ldots \\
\text { Calcium }(\mathrm{Ca}) \ldots \ldots \\
\text { Magnesium }(\mathrm{Mg}) \ldots \ldots \\
\text { Sodium }(\mathrm{Na}) \ldots \ldots \\
\text { Potassium }(\mathrm{K}) \\
\text { Carbonate }(\mathrm{CO}\end{array}$} & \multirow{2}{*}{$\begin{array}{c}3.5 \\
.06 \\
.00 \\
5.4 \\
2.3\end{array}$} & \multirow{3}{*}{$\begin{array}{c}3.2 \\
.06 \\
.00 \\
12 \\
2.4\end{array}$} & $\begin{array}{c}\text { Hardness as CaCO } \\
\text { Total } \\
\text { Noncarbonate..................... }\end{array}$ & $\begin{array}{r}23 \\
7\end{array}$ & $\begin{array}{l}40 \\
21\end{array}$ \\
\hline & & & \multirow{6}{*}{$\begin{array}{l}\text { Color .................... } \\
\text { pH ........................ } \\
\text { (micromhos at } \\
25 \text { C.) ................ } \\
\text { Turbidity .............. } \\
\text { Temperature (F.)... } \\
\text { Date of collection... }\end{array}$} & \multirow{2}{*}{$\begin{array}{r}12 \\
6.8\end{array}$} & \multirow{2}{*}{7.5} \\
\hline & 5.7 & & & & \\
\hline & $\begin{array}{r}0 \\
19\end{array}$ & & & 80.3 & 122 \\
\hline & 6.8 & 18 & & -- & -- \\
\hline & 8.0 & 10 & & & \\
\hline & ${ }_{50}^{.1 .3}$ & $70^{.3}$ & & $\underset{1951}{\operatorname{Jan} .8,}$ & $\begin{array}{c}\text { Jan. 8, } \\
1951\end{array}$ \\
\hline
\end{tabular}

Regular determinations at treatment plant, 1950

\begin{tabular}{|c|c|c|c|c|c|c|c|c|c|c|c|c|}
\hline & \multicolumn{3}{|c|}{$\begin{array}{l}\text { Alkalinity } \\
\text { as } \mathrm{CaCO} \\
\text { (ppm) }\end{array}$} & \multicolumn{3}{|c|}{ pH } & \multicolumn{3}{|c|}{$\begin{array}{l}\text { Hardness } \\
\text { as } \mathrm{CaCO} \\
\text { (ppm) }\end{array}$} & \multicolumn{3}{|c|}{ Turbidity } \\
\hline & Av & Max & Min & Av & Max & Min & Av & Max & Min & Av & $\operatorname{Max}$ & $\operatorname{Min}$ \\
\hline $\begin{array}{l}\text { Raw water......... } \\
\text { Finished water... }\end{array}$ & $\begin{array}{l}24 \\
30\end{array}$ & \begin{tabular}{l|}
32 \\
38
\end{tabular} & \begin{tabular}{|l|}
20 \\
24 \\
\end{tabular} & $\begin{array}{l}6.7 \\
7.1\end{array}$ & $\begin{array}{l}7.0 \\
7.2\end{array}$ & \begin{tabular}{|l|}
6.3 \\
7.0
\end{tabular} & $\begin{array}{l}28 \\
47\end{array}$ & \begin{tabular}{|l|}
31 \\
53
\end{tabular} & \begin{tabular}{|l|}
25 \\
42
\end{tabular} & $\begin{array}{r}150 \\
0\end{array}$ & $\begin{array}{r}961 \\
0\end{array}$ & $\begin{array}{r}34 \\
0\end{array}$ \\
\hline
\end{tabular}




\section{NEW BERN \\ (Population 15, 812)}

Ownership: Municinal; also supplies suburban districts. Total population supplied, about 17,380 .

Source: 8 wells (1 to 8) 90 to $115 \mathrm{ft}$ deep. The total yield of the wells is reported to be $2,100 \mathrm{gpm}$.

Treatment: Zeolite softening, ammoniation, and chlorination.

Rated capacity of treatment plant: 2,000,000 gpd.

Raw-water storage: None.

Finished-water storage: 1 reservoir, 300,000 gal; 1 elevated tank, 100,000 gal.

\section{ANALYSES}

(Analyses, in parts per million, by U. S. Geological Survey)

\begin{tabular}{|c|c|c|c|c|c|}
\hline & $\begin{array}{l}\text { Raw } \\
\text { watera }\end{array}$ & $\begin{array}{r}\text { Finished } \\
\text { water a }\end{array}$ & & $\begin{array}{c}\text { Raw } \\
\text { water a }\end{array}$ & $\begin{array}{c}\text { Finished } \\
\text { water a }\end{array}$ \\
\hline $\begin{array}{l}\text { Silica }\left(\mathrm{SiO}_{2}\right) \ldots \ldots \ldots \ldots \\
\text { Iron }(\mathrm{Fe}) \ldots \ldots \ldots \ldots . . . . . . \\
\text { Manganese }(\mathrm{Mn})\end{array}$ & $\begin{array}{l}16 \\
1.5 \\
.00\end{array}$ & $\begin{array}{r}17 \\
.18 \\
.00\end{array}$ & $\begin{array}{r}\text { Hardness as } \mathrm{CaCO}_{3} \text { : } \\
\text { Total } . . . . . . . . . . . . . . . . \\
\text { Noncarbonate...... }\end{array}$ & $\begin{array}{r}195 \\
20\end{array}$ & $\begin{array}{r}66 \\
0\end{array}$ \\
\hline 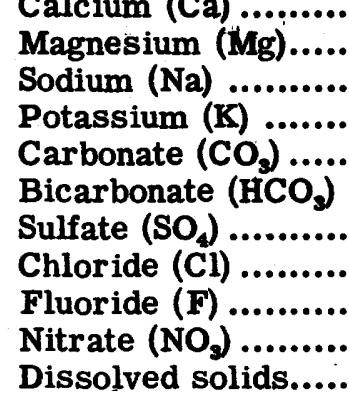 & $\begin{array}{r}75 \\
1.8 \\
4.5 \\
0 \\
213 \\
19 \\
7.0 \\
.0 \\
.1\end{array}$ & $\begin{array}{r}25 \\
.9 \\
67 \\
0 \\
219 \\
15 \\
12 \\
.0 \\
244^{.1}\end{array}$ & $\begin{array}{l}\text { Color .................... } \\
\text { pH .......................... } \\
\text { Specific conductance } \\
\text { (micromhos at } \\
25 \text { C. )................ } \\
\text { Turbidity ............. } \\
\text { Temperature (F.)... } \\
\text { Date of collection ... }\end{array}$ & $\begin{array}{r}418 \\
1 \\
60 \\
\text { Aug. } 30, \\
1951\end{array}$ & $\begin{array}{r}411 \\
0 \\
60 \\
\text { Aug. 30, } \\
1951\end{array}$ \\
\hline
\end{tabular}

a Mixed sample from 8 wells. 


\section{RALEIGH \\ (Population, 65, 679)}

Ownership: Municipal; also supplies suburban districts. Total population supplied, about 70,000.

Source: Walnut Creek impounded in Lakes Raleigh and Johnson. Swift Creek impounded by mill.dam, auxiliary supply (used extensively during periods of drought).

Treatment: Coagulation with alum, ammoniation (ammonium sulfate), prechlorination, sedimentation, activated carbon, rapid sand filtration, adjustment of $\mathrm{pH}$ with lime, and postchlorination.

Rated capacity of treatment plant: 8,000, 000 gpd.

Raw-water storage: Lake Raleigh, 150, 000, 000 gal; Lake Johnson, 500, 000, 000 gal; Swift Creek impounded, 20, 000, 000 gal.

Finished-water storage: 2 clear wells, 2, 000, 000 and 2, 400, 000 gal; 3 elevated tanks, 100,$000 ; 600,000 ; 750,000 \mathrm{gal}$.

The treatment plant is on the old Fayetteville Road 1 mile south of the city.

\section{ANALYSES}

(Analyses, in parts per million, by U. S. Geological Survey)

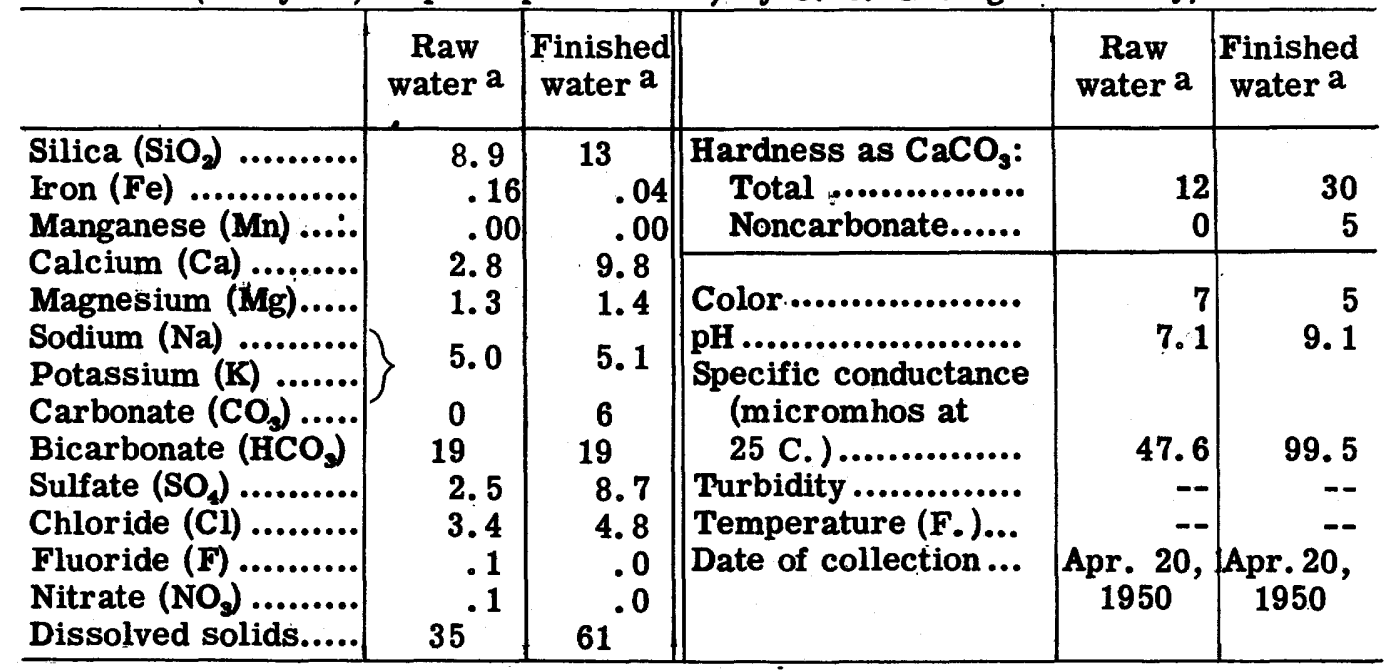

Regular determinations at treatment plant, 1950

\begin{tabular}{|c|c|c|c|c|c|c|c|c|c|c|c|c|}
\hline & \multicolumn{3}{|c|}{$\begin{array}{l}\text { Alkalinity } \\
\text { as } \mathrm{CaCO}_{3} \\
\text { (ppm) }\end{array}$} & \multicolumn{3}{|c|}{ pH } & \multicolumn{3}{|c|}{$\begin{array}{c}\text { Hardness } \\
\text { as } \mathrm{CaCO}_{3} \\
\text { (ppm) }\end{array}$} & \multicolumn{3}{|c|}{ Turbidity } \\
\hline & Av & $\operatorname{Max}$ & Min & Av & Max & Min & Av & Max & Min & Av & $\operatorname{Max}$ & Min \\
\hline $\begin{array}{l}\text { Raw water.......... } \\
\text { Finished water... }\end{array}$ & $\begin{array}{l}14 \\
27\end{array}$ & \begin{tabular}{|l|}
34 \\
39
\end{tabular} & $\begin{array}{l}8.8 \\
0.9\end{array}$ & $\begin{array}{l}7.0 \\
9.2\end{array}$ & $\begin{array}{l}8.3 \\
9.5\end{array}$ & \begin{tabular}{|l}
6.3 \\
8.4
\end{tabular} & -- & -- & -- & $\begin{array}{r}11 \\
.18\end{array}$ & $\begin{array}{l}115 \\
.75 \\
\end{array}$ & $\begin{array}{r}4.0 \\
.0\end{array}$ \\
\hline
\end{tabular}

${ }^{\mathrm{a}}$ Walnut Creek. 


\section{REIDSVILLE}

(Population, 11, 708)

Ownership: Municipal; also supplies other suburban districts. Total population supplied, about 16,000 .

Source: Troublesome Creek. The intake is about 3 miles southwest of treatment plant.

Treatment: Aeration (air-mix), coagulation with alum, sedimentation, rapid sand filtration, postchlorination, and adjustment of $\mathrm{pH}$ with soda ash.

Rated capacity of treatment plant: 1,500,000 gpd.

Raw-water storage: None.

Finished-water storage: 1 clear well, 1, 000, 000 gal; 1 standpipe, 790, 000 gal; 2 elevated tanks, 500, 000 and 75, 000 gal.

The treatment plant is 3 miles west of the center of the city. The capacity of treatment plant is to be increased to $3,000,000$ gpd by 1953 . Prechlorination and fluoridation will begin in 1953.

\section{ANALYSES}

(Analyses, in parts per million, by U. S. Geological Survey)

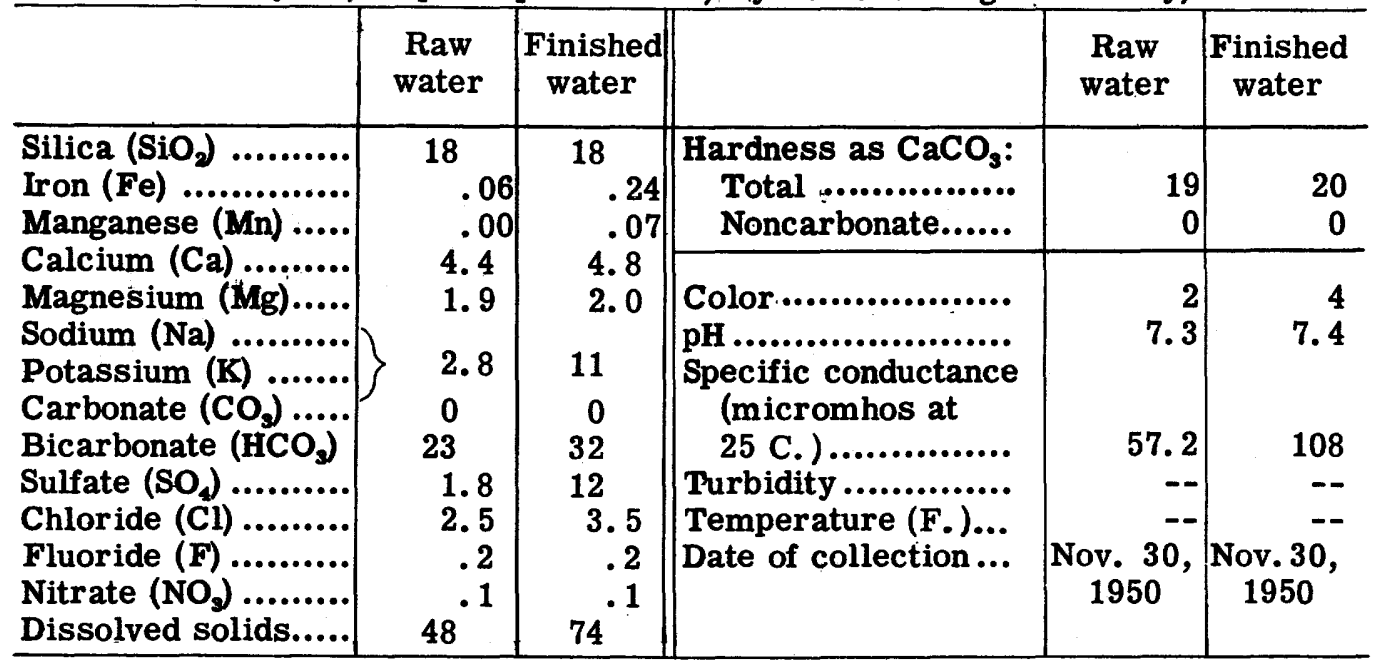

Regular determinations at treatment plant, 1950

\begin{tabular}{|c|c|c|c|c|c|c|c|c|c|c|c|c|}
\hline & \multicolumn{3}{|c|}{$\begin{array}{l}\text { Alkalinity } \\
\text { as } \mathrm{CaCO}{ }_{3} \\
\text { (ppm) }\end{array}$} & \multicolumn{3}{|c|}{ pH } & \multicolumn{3}{|c|}{$\begin{array}{c}\text { Hardness } \\
\text { as } \mathrm{CaCO}_{3} \\
\text { (ppm) }\end{array}$} & \multicolumn{3}{|c|}{ Turbidity } \\
\hline & $\mathbf{A v}$ & $\operatorname{Max}$ & Min & Av & Max & Min & Av & Max & Min & Av & Max & Min \\
\hline $\begin{array}{l}\text { Raw water.......... } \\
\text { Finished water... }\end{array}$ & $\begin{array}{l}25 \\
36\end{array}$ & $\begin{array}{l}32 \\
48\end{array}$ & $\begin{array}{l}18 \\
22\end{array}$ & $\begin{array}{l}6.8 \\
8.1\end{array}$ & $\begin{array}{l}7.1 \\
8.6\end{array}$ & $\begin{array}{l}6.5 \\
7.6\end{array}$ & $\begin{array}{l}18 \\
18\end{array}$ & $\begin{array}{l}19 \\
19\end{array}$ & $\begin{array}{l}17 \\
17\end{array}$ & $\begin{array}{r}33 \\
<1\end{array}$ & $\begin{array}{l}400 \\
<1\end{array}$ & $\begin{array}{r}5 \\
<1 \\
\end{array}$ \\
\hline
\end{tabular}




\section{ROCKY MOUNT \\ (Population, 27, 697)}

Ownership: Municipal; also supplies suburban districts. Total population supplied about 36,000 .

Source: Tar River. The intake is about $400 \mathrm{ft}$ west of treatment plant, which is at the edge of the city.

Treatment: Coagulation with alum and lime, carbon, sedimentation, rapid sand filtration, chlorination, Calgon, ammoniation, and adjustment of $\mathrm{pH}$ with lime.

Rated capacity of treatment plant: $6,750,000 \mathrm{gpd}$.

Raw-water storage: None.

Finished-water storage: 2 reservoirs 500,000 and 1,500, 000 gal; 1 elevated tank, $1,000,000 \mathrm{gal}$.

The river was at high stage and more turbid than usual at the time of the collection of the samples, and the samples are not typical of average conditions.

\section{ANALYSES}

(Analyses, in parts per million, by U. S. Geological Survey)

\begin{tabular}{|c|c|c|c|c|c|}
\hline & $\begin{array}{l}\text { Raw } \\
\text { water }\end{array}$ & $\begin{array}{c}\text { Finished } \\
\text { water }\end{array}$ & & $\begin{array}{c}\text { Raw } \\
\text { water }\end{array}$ & $\begin{array}{c}\text { Finished } \\
\text { water }\end{array}$ \\
\hline $\begin{array}{l}\text { Silica }\left(\mathrm{SiO}_{2}\right) \\
\text { Iron }(\mathrm{Fe}) \ldots \ldots \ldots \\
\text { Manganese }(\mathrm{Mn}) \ldots \ldots\end{array}$ & \multirow{2}{*}{$\begin{array}{r}11 \\
.06 \\
.00 \\
4.2 \\
1.8\end{array}$} & \multirow{2}{*}{$\begin{array}{r}16 \\
.04 \\
15 \\
.00 \\
2.0\end{array}$} & 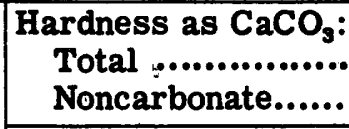 & $\begin{array}{r}18 \\
3\end{array}$ & $\begin{array}{l}46 \\
23\end{array}$ \\
\hline Magnesium (Mg)..... & & & \multirow{7}{*}{ 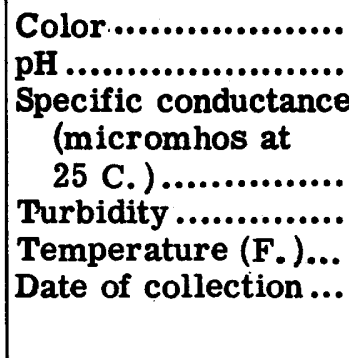 } & 33 & 3 \\
\hline $\begin{array}{l}\text { Sodium (Na) } \ldots \ldots \ldots \ldots \\
\text { Potassium }(\ddot{K}) \ldots \ldots\end{array}$ & 3.2 & 5.4 & & 6.6 & 7.4 \\
\hline $\begin{array}{l}\text { Carbonate }\left(\mathrm{CO}_{3}\right) \ldots . . . \\
\text { Bicarbonate }\left(\mathrm{HCO}_{3}\right)\end{array}$ & & & & 57.3 & 129 \\
\hline Sulfate $\left(\mathrm{SO}_{4}\right) \ldots \ldots \ldots$ & 5.1 & 26 & & -- & -- \\
\hline Chloride (Cl) .......... & 3.1 & 4.8 & & & -- \\
\hline Fluoride (F) ............ & .0 & & & June 7, & June 7 , \\
\hline $\begin{array}{l}\text { Nitrate }\left(\mathrm{NO}_{2}\right) \ldots . . . . . . . \\
\text { Dissolved solids..... }\end{array}$ & $51^{.5}$ & $86^{.5}$ & & & 1950 \\
\hline
\end{tabular}


SALISBURY

(Population, 20, 102)

Ownership: Municipal; also supplies Spencer and East.Spencer anä other suburban districts. Tatal population supplied, about 26,600.

Source: Yadkin River. Grant Creek is used in extreme emergency. . The intake is at the junction of 'Yadkin and South Yadkin Rivers about 8 miles from the treatment plant which is on Kerr Street in the city.

Treatment: Prechlorination at raw water reservoir and at plant (breakpoint), coagulation with alum, sedimentation, rapid sand filtration, and adjustment of pH with lime.

Rated capacity of treatment plant: 4,000,000 gpd.

Raw-water storage: Reservoir (near river), 8,000, 000 gal.

Finished-water storage: 2 clear wells, 750, 000 and 1,000,000 gal; 2 elevated tanks, 1, 000, 000 and 250, 000 gal.

\section{ANALYSES}

(Analyses, in parts per million, by U. S. Geological Survey)

\begin{tabular}{|c|c|c|c|c|c|c|c|c|c|c|c|c|}
\hline & \multicolumn{2}{|r|}{$\begin{array}{c}\text { Raw } \\
\text { water }\end{array}$} & \multicolumn{2}{|c|}{$\begin{array}{c}\text { Finished } \\
\text { water }\end{array}$} & \multicolumn{4}{|c|}{ s } & \multicolumn{2}{|c|}{$\begin{array}{l}\text { Raw } \\
\text { water }\end{array}$} & \multicolumn{2}{|c|}{$\begin{array}{c}\text { Finished } \\
\text { water }\end{array}$} \\
\hline \multirow{7}{*}{\multicolumn{2}{|c|}{ 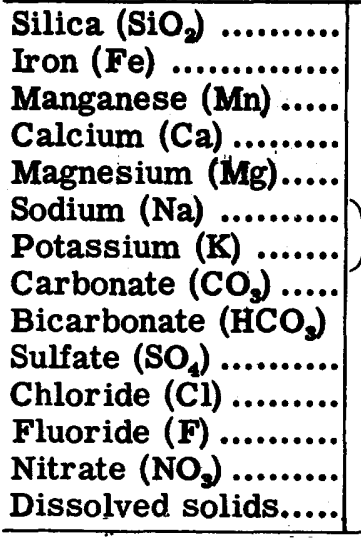 }} & \multirow{2}{*}{$\begin{array}{r}12 \\
.05 \\
.00 \\
3.2 \\
1.3\end{array}$} & \multirow{2}{*}{\multicolumn{2}{|c|}{\begin{tabular}{|c||}
12 \\
.03 \\
.00 \\
12 \\
1.3
\end{tabular}}} & \multicolumn{4}{|c|}{$\begin{array}{c}\text { Hardness as } \mathrm{CaCO}_{3}: \\
\text { Total } \\
\text { Noncarbonate..................... }\end{array}$} & & \multicolumn{2}{|r|}{$\begin{array}{l}35 \\
12\end{array}$} \\
\hline & & & & & \multirow{6}{*}{\multicolumn{4}{|c|}{$\begin{array}{l}\text { Color.................... } \\
\text { pH ....................... } \\
\text { Specific conductance } \\
\text { (micromhos at } \\
25 \text { C.) ................ } \\
\text { Turbidity ............. } \\
\text { Temperature (F.)... } \\
\text { Date of collection ... }\end{array}$}} & \multirow{2}{*}{\multicolumn{2}{|c|}{$\begin{array}{r}4 \\
7.1\end{array}$}} & \multirow{2}{*}{\multicolumn{2}{|c|}{$\begin{array}{r}6 \\
8.4\end{array}$}} \\
\hline & & 3. 7 & \multicolumn{2}{|r|}{3.4} & & & & & & & & \\
\hline & & & & & & & & \multicolumn{2}{|r|}{55.8} & \multicolumn{2}{|c|}{91.5} \\
\hline & & 2.7 & & & & & & & & - & & - \\
\hline & & 2.2 & & 4.2 & & & & & & & & \\
\hline & & $35^{.1}$ & & .1 & & & & & \multicolumn{2}{|c|}{$\begin{array}{c}\text { June 8, } \\
1950\end{array}$} & \multicolumn{2}{|c|}{$\begin{array}{c}\text { June 8, } \\
1950\end{array}$} \\
\hline \multicolumn{13}{|c|}{ Regular determinations at treatment plant, 1950} \\
\hline & \multicolumn{3}{|c|}{$\begin{array}{l}\text { Alkalinity } \\
\text { as CaCO } \\
\text { (ppm) }\end{array}$} & \multicolumn{3}{|c|}{ pH } & \multicolumn{3}{|c|}{$\begin{array}{l}\text { Hardness } \\
\text { as } \mathrm{CaCO}_{3} \\
\text { (ppm) }\end{array}$} & \multicolumn{3}{|c|}{ Turbidity } \\
\hline & Av & $\operatorname{Max}$ & Min & Av & Max & $\operatorname{Min}$ & Av & $\operatorname{Max}$ & Min & $\mathbf{A v}$ & $\operatorname{Max}$ & Min \\
\hline Raw water.......... & 15 & 16 & 14 & 7.0 & 7.2 & 6.9 & 16 & -- & -1 & 133 & 291 & 36 \\
\hline Finished water... & 23 & 26 & 19 & 8.4 & 8.9 & 7.7 & 30 & -- & $\ldots$ & 0 & 0 & \\
\hline
\end{tabular}




\section{SANFORD}

(Population, 10, 013)

Ownership: Municipal; also supplies suburban districts. Total population supplied, about 10,400 .

Source: Partridge Creek impounded in 2 lake reservoirs near treatment plant; Lick Creek impounded in Lake William.

Treatment: Prechlorination, coagulation with lime and alum, sedimentation, rapid sand filtration, postchlorination, Calgon, and final adiustment of $\mathrm{pH}$ with lime.

Rated capacity of treatment plant: $1,500,000$ gpd.

Raw - water storage: 3 lake reservoirs about $18,000,000,75,100,000$ and $35,000,000$ gal.

Finished-water storage: 1 clear well, ,350,000 gal; 1 standpipe, 250, 000 gal; 1 elevated tank, 75, 000 gal.

Lake William is about 8 miles southeast of the center of town. Water is pumped from Lake William into Partridge Creek, which is impounded in reservoirs at the treatment plant.

\section{ANALYSES}

(Analyses, in parts per million, by U. S. Geological Survey)

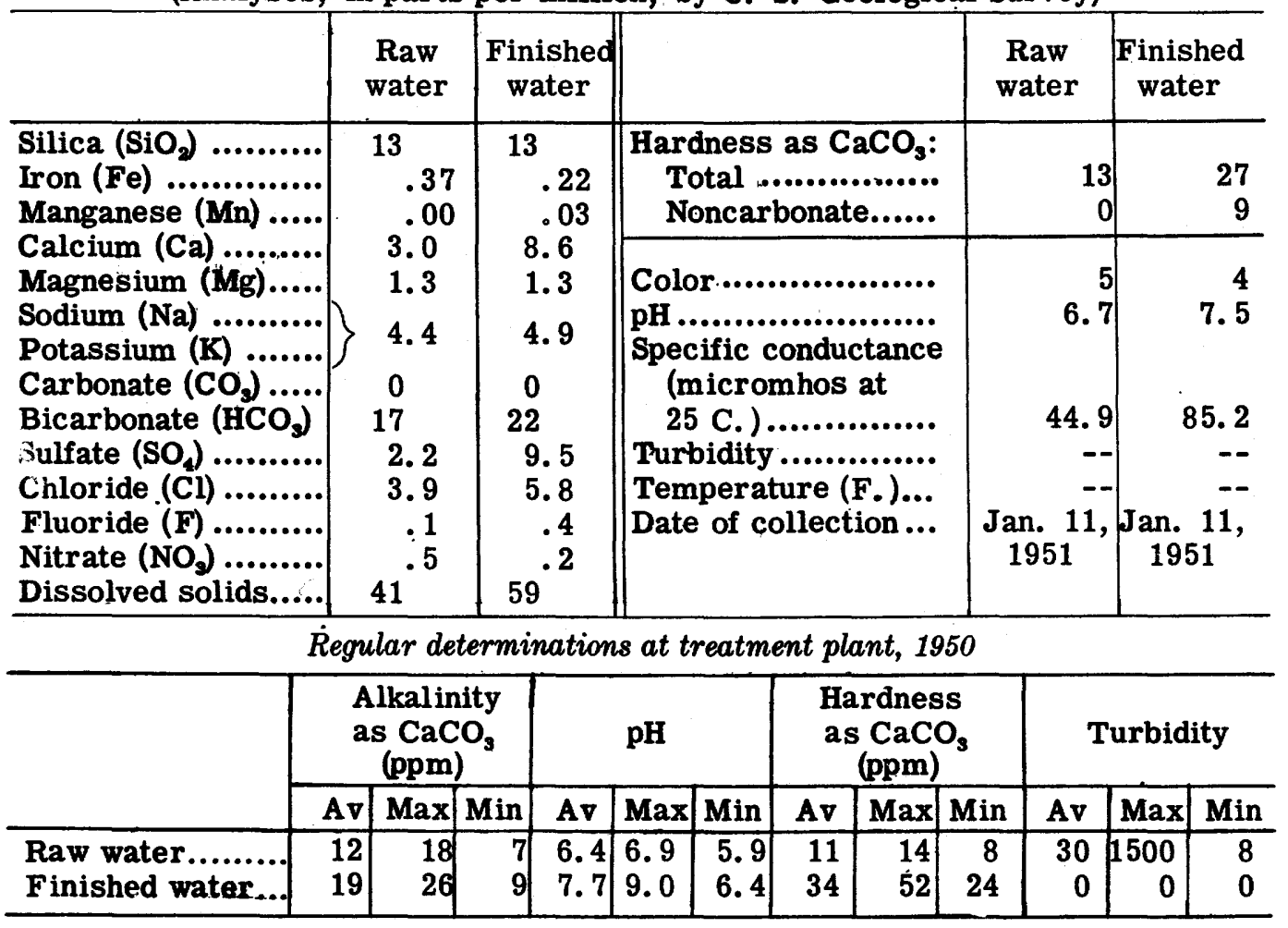




\section{SHELBY \\ (Population, 15, 508)}

Ownership: Municipal; also supplies suburban districts. Total population supplied, about 16,000 .

Source: First Broad River. The intake is on the River about 0.2 mile northwest of treatment plant, which is about $1 \frac{1}{4}$ miles northwest of the center of the city.

Treatment: Copper sulfate, prechlorination, coagulation with alum and lime, ammoniation, carbon, sedimentation, rapid sand filtration, postchlorination, and final adjustment of $\mathrm{pH}$ with lime.

Rated capacity of treatment plant: 2, 000, $000 \mathrm{gpd}$.

Raw-water storage: 2 reservoirs, 8,500, 000 and 5, 000, 000 gal.

Finished-water storage: 1 clear well, 2, 000,000 gal; 2 elevated tanks, each 500,000 gal.

\section{ANALYSES}

(Analyses, in parts per million, by U. S. Geological Survey)

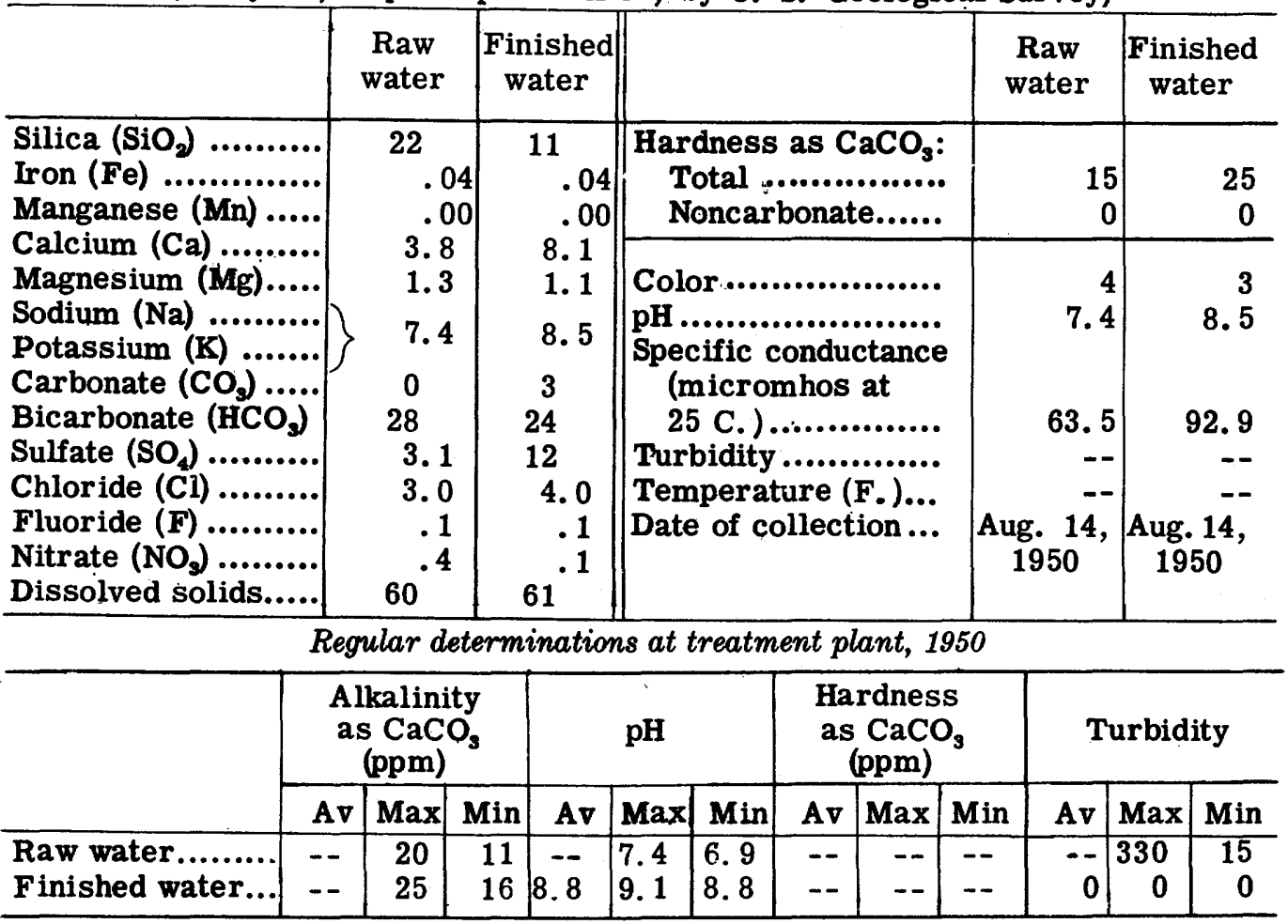




\section{STATESVILLE \\ (Population, 16, 901)}

Ownership: Municipal: also supplies suburban districts. Total population supplied, about 18,600 .

Source: Fourth Creek. Morrison Creek, emergency supply. The intake is about $1,600 \mathrm{ft}$ north of the treatment plant, which is approximately 2 miles north of the city.

Treatment: Prechlorination, coagulation with alum and lime, activated carbon . (if needed), sedimentation, rapid sand filtration, postchlorination (if needed), Calgon, and adjustment of $\mathrm{pH}$ with lime.

Rated capacity of treatment plant: $3,000,000 \mathrm{gpd}$.

Raw-water storage: None.

Finished-water storage: 1 elevated tank 1, 000, 000 gal; clear well, 1, 100, 000 gal.

\section{ANALYSES}

(Analyses, in parts per million, by U. S. Geological Survey)

\begin{tabular}{|c|c|c|c|c|c|}
\hline & $\begin{array}{l}\text { Raw } \\
\text { water }\end{array}$ & $\begin{array}{c}\text { Finished } \\
\text { water }\end{array}$ & & $\begin{array}{c}\text { Raw } \\
\text { water }\end{array}$ & $\begin{array}{c}\text { Finished } \\
\text { water }\end{array}$ \\
\hline $\begin{array}{l}\text { Silica }\left(\mathrm{SiO}_{2}\right) \ldots \ldots \ldots \ldots \\
\text { Iron }(\mathrm{Fe}) \ldots \ldots \ldots \ldots \\
\text { Manganese }\left(\mathrm{Mn}^{2} \ldots \ldots \ldots\right.\end{array}$ & $\begin{array}{r}20 \\
.07 \\
.00\end{array}$ & $\begin{array}{r}18 \\
.07 \\
.00\end{array}$ & 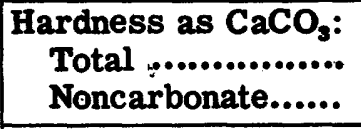 & $\begin{array}{r}23 \\
0 \\
\end{array}$ & $\begin{array}{r}36 \\
9 \\
\end{array}$ \\
\hline 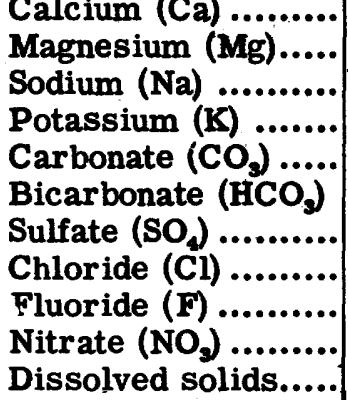 & $\begin{array}{r}5.2 \\
2.5 \\
4.6 \\
0 \\
34 \\
1.9 \\
2.1 \\
.1 \\
.2\end{array}$ & $\begin{array}{c}10 \\
2.6 \\
5.0 \\
0 \\
32 \\
14 \\
3.4 \\
.3 \\
.2\end{array}$ & $\begin{array}{l}\text { Color } \ldots . . . . . . . . . . . . . . \\
\text { pH ....................... } \\
\text { Specific conductance } \\
\text { (micromhos at } \\
25 \text { C. }) . . . . . . . . . . . . . \\
\text { Turbidity ............. } \\
\text { Temperature (F.)... } \\
\text { Date of collection... }\end{array}$ & $\begin{array}{r}66.8 \\
\text { Mar. } 27, \\
1951\end{array}$ & $\begin{array}{r}99.9 \\
- \\
- \\
1951\end{array}$ \\
\hline
\end{tabular}

Regular determinations at treatment plant, 1950

\begin{tabular}{l|c|c|c|c|c|c|c|c|c|c|c|c}
\hline & \multicolumn{3}{|c|}{$\begin{array}{c}\text { Alkalinity } \\
\text { as CaCO } \\
\text { (ppm) }\end{array}$} & \multicolumn{3}{|c|}{ pH } & \multicolumn{3}{c|}{$\begin{array}{c}\text { Hardness } \\
\text { as CaCO } \\
\text { (ppm) }\end{array}$} & \multicolumn{3}{c}{ Turbidity } \\
\cline { 2 - 14 } & Av & Max & Min & Av & Max & Min & Av & Max & Min & Av & Max & Min \\
\hline Raw water......... & 28 & 29 & 27 & 7.1 & 7.2 & 6.9 & -- & -- & -- & 37 & 2000 & 20 \\
Finished water... & 28 & 29 & 27 & 7.1 & 7.2 & 6.9 & 40 & 42 & 39 & .1 & 2.0 & 0 \\
\hline
\end{tabular}




\section{THOMASVILLE \\ (Population, 11, 154)}

Ownership: Municipal; also supplies suburban districts. Total population supplied, about 14,500 .

Source: Abbotts Creek. The intake is $4 \frac{1}{4}$ miles northwest of the treatment plant, which is $1 \frac{1}{2}$ miles west of the center of the city on U. S. Highway 70 .

Treatment: Prechlorination, coagulation with alum and lime, sedimentation, rapid sand filtration, postchlorination at times, and adjustment of $\mathrm{pH}$ with lime.

Rated capacity of treatment plant: 1,500,000 gpd.

Raw-water storage: None.

Finished-water storage: 1 clear well, 480,000 gal; 1 elevated tank, 1, 000, 000 gal.

\section{ANALYSES}

(Analyses, in parts per million, by U. S. Geological Survey)

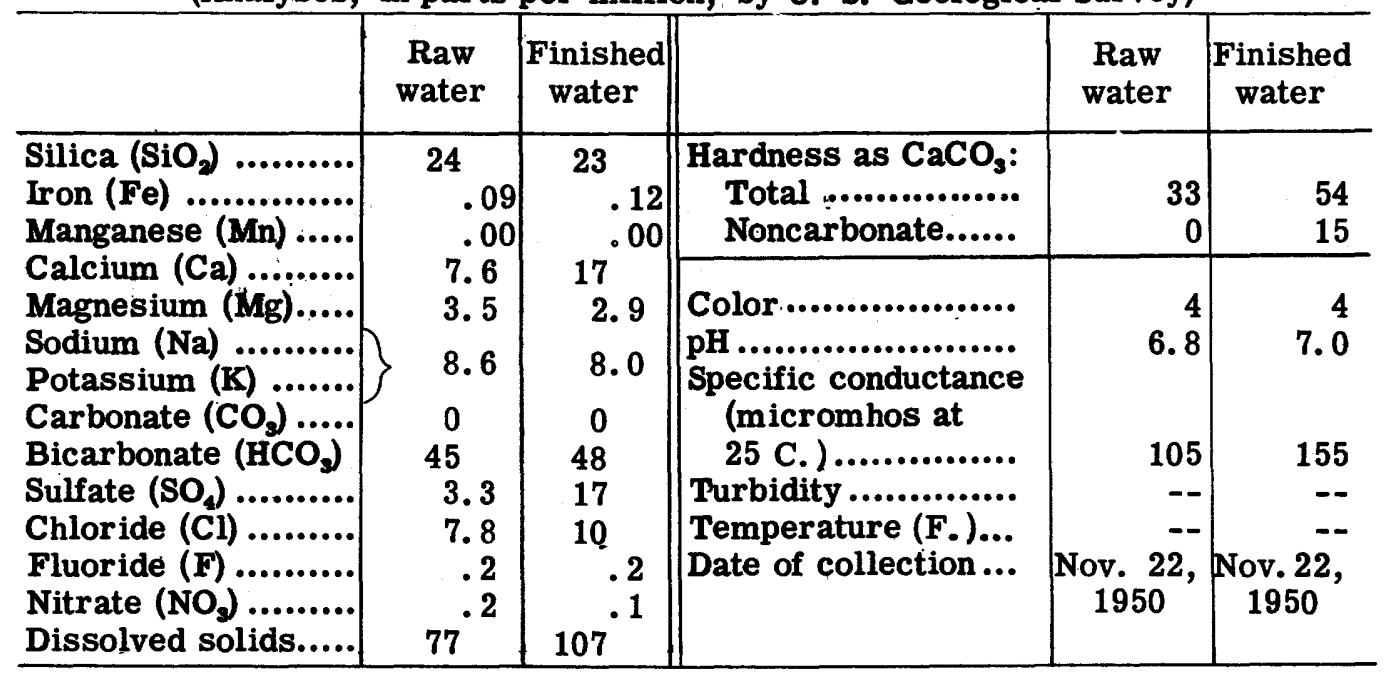

Regular determinations at treatment plant, 1950

\begin{tabular}{|c|c|c|c|c|c|c|c|c|c|c|c|c|}
\hline & \multicolumn{3}{|c|}{$\begin{array}{c}\text { Alkalinity } \\
\text { as CaCO } \\
\text { (ppm) }\end{array}$} & \multicolumn{3}{|c|}{ pH } & \multicolumn{3}{|c|}{$\begin{array}{l}\text { Hardness } \\
\text { as } \mathrm{CaCO} \\
\text { (ppm) }\end{array}$} & \multicolumn{3}{|c|}{ Turbidity } \\
\hline & Av & Max & Min & Av & Max & Min & Av & Max & Min & Av & Max & Min \\
\hline $\begin{array}{l}\text { Raw water......... } \\
\text { Finished water... }\end{array}$ & $\begin{array}{l}34 \\
38\end{array}$ & \begin{tabular}{|l|}
39 \\
45 \\
\end{tabular} & $\begin{array}{l}26 \\
28\end{array}$ & $\begin{array}{l}7.1 \\
8.1\end{array}$ & $\begin{array}{l}7.3 \\
8.9\end{array}$ & $\begin{array}{l}6.9 \\
7.2\end{array}$ & -- & -- & - & $\begin{array}{r}210 \\
--\end{array}$ & $\begin{array}{r}1093 \\
--\end{array}$ & $\begin{array}{r}138 \\
--\end{array}$ \\
\hline
\end{tabular}




\section{WILSON \\ (Population, 23, 010)}

Ownership: Municipal; also supplies suburban districts. Total population supplied, about 24,000 .

Source: Contentnea Creek, 75 percent of supply; Toisnot Swamp (Creek), 25 percent. The intake on Contentnea Creek is about 4 miles southwest of treatment plant and the intake on Toisnot Swamp is about 0.5 mile east.

Treatment: Prechlorination, coagulation with alum, lime (if necessary), sedimentation, rapid sand filtration, ammoniation, Calgon, postchlorination, and adjustment of $\mathrm{pH}$ with lime.

Rated capacity of treatment plant: 3,000,000 gpd.

Raw-water storage: None.

Finished-water storage: 1 reservoir, 2, 000, 000 gal; 1 elevated tank, 1, 000, 000 gal.

During dry periods, Toisnot Swamp (Creek) may furnish about 35 percent of the total raw-water supply. Plans are under way for increasing the treatment plant capacity to $6,000,000$ gpd.

\section{ANALYSES}

(Analyses, in parts per million, by U. S. Geological Survey)

\begin{tabular}{|c|c|c|c|c|c|}
\hline & $\begin{array}{c}\text { Raw } \\
\text { water }\end{array}$ & $\begin{array}{c}\text { Finished } \\
\text { water }\end{array}$ & & $\begin{array}{c}\text { Raw } \\
\text { water }\end{array}$ & $\begin{array}{c}\text { Finished } \\
\text { water }\end{array}$ \\
\hline $\begin{array}{l}\text { Silica }\left(\mathrm{SiO}_{2}\right) \\
\text { Iron }(\mathrm{Fe}) \\
\text { Manganese }(\mathrm{Mn})\end{array}$ & $\begin{array}{l}7.6 \\
.21 \\
.00\end{array}$ & $\begin{array}{l}8.3 \\
.06 \\
.00\end{array}$ & $\begin{array}{c}\text { Hardness as } \mathrm{CaCO}_{3}: \\
\text { Total } \\
\text { Noncarbonate................... }\end{array}$ & $\begin{array}{r}10 \\
0\end{array}$ & $\begin{array}{l}41 \\
13\end{array}$ \\
\hline 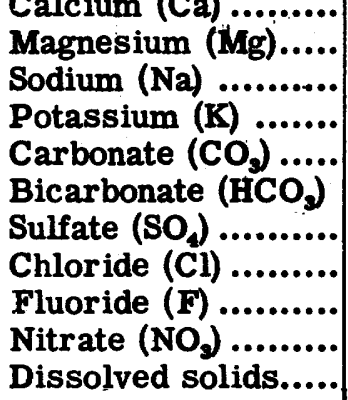 & $\begin{array}{r}2.3 \\
1.1 \\
5.6 \\
0 \\
15 \\
2.9 \\
4.9 \\
.0 \\
.3\end{array}$ & $\begin{array}{c}14 \\
1.4 \\
13 \\
0 \\
34 \\
30 \\
6.5 \\
.1 \\
.8 \\
92\end{array}$ & $\begin{array}{l}\text { Color } \ldots . . . . . . . . . . . . . . \\
\text { pH ........................ } \\
\text { Specific conductance } \\
\text { (micromhos at } \\
25 \text { C. ) ................ } \\
\text { Turbidity .............. } \\
\text { Temperature (F.)... } \\
\text { Date of collection ... }\end{array}$ & $\begin{array}{r}48.1 \\
\text { June } 14, \\
1950\end{array}$ & $\begin{array}{r}155 \\
1950\end{array}$ \\
\hline
\end{tabular}

Regular determinations at treatment plant, 1950

\begin{tabular}{l|r|r|r|r|r|r|r|r|r|r|r|r}
\hline & \multicolumn{3}{|c|}{$\begin{array}{c}\text { Alkalinity } \\
\text { as CaCO, } \\
\text { (ppm) }\end{array}$} & \multicolumn{3}{|c|}{ pH } & \multicolumn{3}{c|}{$\begin{array}{c}\text { Hardness } \\
\text { as CaCO } \\
\text { (ppm) }\end{array}$} & \multicolumn{3}{c}{ Turbidity } \\
\cline { 2 - 14 } & Av & Max & Min & Av & Max & Min & Av & Max & Min & Av & Max & Min \\
\hline Raw water......... & 16 & 24 & 12 & 6.8 & 7.2 & 6.4 & 20 & 38 & 10 & 5 & 300 & 0 \\
Finished water... & 26 & 30 & 16 & $\mathbf{8 . 2}$ & $\mathbf{9 . 2}$ & 7.2 & 36 & 54 & 20 & 0 & 0 & 0 \\
\hline
\end{tabular}




\section{WILMINGTON}

(Population, 45, 043)

Ownership: Municipal; also supplies suburban districts. Total population supplied, about 48,500 .

Source: Cape Fear River. Northeast Cape Fear River and Toomers Creek auxiliary or emergency supplies (neither used in last five years). The intake is approximately 30 miles north of Wilmington at Kings Bluff.

Treatment: Coagulation with alum, sedimentation, rapid sand filtration, ammoniation, chlorination, and adjustment of $\mathrm{pH}$ with lime.

Rated capacity of treatmenit plant: 7,000,000 gpd.

Raw-water storage: None.

Finished-water storage: 2 reservoirs, 4,000,000 and 1, 000,000 gal; 2 elevated tanks, 500, 000 and 146, 000 gal.

\section{ANALYSES}

(Analyses, in parts per million, by U. S. Geological Survey)

\begin{tabular}{|c|c|c|c|c|c|}
\hline & $\begin{array}{c}\text { Raw } \\
\text { water }\end{array}$ & $\begin{array}{c}\text { Finished } \\
\text { water }\end{array}$ & & $\begin{array}{c}\text { Raw } \\
\text { water }\end{array}$ & $\begin{array}{c}\text { Finished } \\
\text { water }\end{array}$ \\
\hline \multirow{8}{*}{ 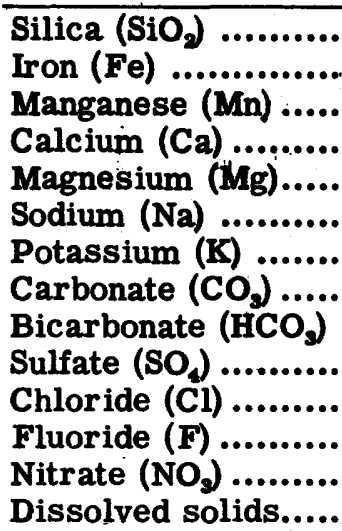 } & \multirow{2}{*}{$\begin{array}{l}7.4 \\
.02 \\
.00 \\
4.2 \\
1.5\end{array}$} & \multirow{2}{*}{$\begin{array}{c}6.1 \\
.05 \\
.00 \\
11 \\
1.7\end{array}$} & $\begin{array}{r}\text { Hardness as } \mathrm{CaCO}_{\mathbf{3}} \text { : } \\
\text { Total } \\
\text { Noncarbonate.................. }\end{array}$ & $\begin{array}{r}17 \\
0\end{array}$ & $\begin{array}{r}34 \\
9\end{array}$ \\
\hline & & & \multirow{7}{*}{$\begin{array}{l}\text { Color................... } \\
\text { pH ......................... } \\
\text { Specific conductance } \\
\text { (micromhos at } \\
25 \text { C.) ............... } \\
\text { Turbidity ............. } \\
\text { Temperature (F.)... } \\
\text { Date of collection ... }\end{array}$} & \multirow{2}{*}{$\begin{array}{r}9 \\
7.1\end{array}$} & \multirow{2}{*}{7.8} \\
\hline & 9.2 & 9.1 & & & \\
\hline & & & & 74.2 & 116 \\
\hline & 6.2 & 17 & & -- & - \\
\hline & 6.6 & 7.6 & & & \\
\hline & $\begin{array}{r}.1 \\
.3\end{array}$ & $\begin{array}{r}.1 \\
.2\end{array}$ & & $\begin{array}{c}\text { Mar. 20, } \\
1950\end{array}$ & $\begin{array}{c}\text { Mar. 20, } \\
1950\end{array}$ \\
\hline & 48 & 68 & & & \\
\hline
\end{tabular}

Regular determinations at treatment plant, 1950

\begin{tabular}{|c|c|c|c|c|c|c|c|c|c|c|c|c|}
\hline & \multicolumn{3}{|c|}{$\begin{array}{c}\text { Alkalinity } \\
\text { as CaCO, } \\
\text { (ppm) }\end{array}$} & \multicolumn{3}{|c|}{ pH } & \multicolumn{3}{|c|}{$\begin{array}{c}\text { Hardness } \\
\text { as CaCO } \\
\text { (ppm) }\end{array}$} & \multicolumn{3}{|c|}{ Turbidity } \\
\hline & $\mathbf{A v}$ & $\operatorname{Max}$ & Min & Av & $\operatorname{Max}$ & Min & Av & $\operatorname{Max}$ & Min & $\mathbf{A v}$ & $\operatorname{Max}$ & Min \\
\hline $\begin{array}{l}\text { Raw water......... } \\
\text { Finished water... }\end{array}$ & $\begin{array}{l}19 \\
28\end{array}$ & $\begin{array}{l}28 \\
34\end{array}$ & $\begin{array}{l}10 \\
21\end{array}$ & $\begin{array}{l}6.9 \\
8.0\end{array}$ & $\begin{array}{l}7.3 \\
8.9\end{array}$ & $\begin{array}{l}6.3 \\
6.9\end{array}$ & $\begin{array}{l}23 \\
42\end{array}$ & $\begin{array}{l}28 \\
50\end{array}$ & $\begin{array}{l}16 \\
30\end{array}$ & $\begin{array}{r}60 \\
0\end{array}$ & $\begin{array}{r}140 \\
0\end{array}$ & $\begin{array}{r}20 \\
0\end{array}$ \\
\hline
\end{tabular}


WINSTON-SALEM

(Population, 87, 811)

Ownership: Municipal; also supplies suburban districts. Total population supplied, about 100, 000 .

Source: Salem and Walker Creeks impounded in Salem Lake. The Yadkin River serves as an auxiliary or emergency supply, furnishing an additional 25, 000, $000 \mathrm{gpd}$. The intake on Salem Lake is 2 miles east of the treatment plant which is about $2 \frac{1}{2}$ miles northeast of the center of the city.

Treatment: Prechlorination, coagulation with alum, sedimentation, rapid sand filtration, postchlorination, fluoridation, Calgon for corrosion control, and adjustment of $\mathrm{pH}$ with lime.

Rated capacity of treatment plant: 20,000,000 gpd.

Raw-water storage: Salem Lake, 1,250,000,000 gal.

Finished-water storage: 3 clear wells, 2,000,000 gal each; 3 elevated tanks, $1,000,000,200,000$, and 50,000 gal.

\section{ANALYSES}

(Analyses, in parts per million, by U. S. Geological Survey)

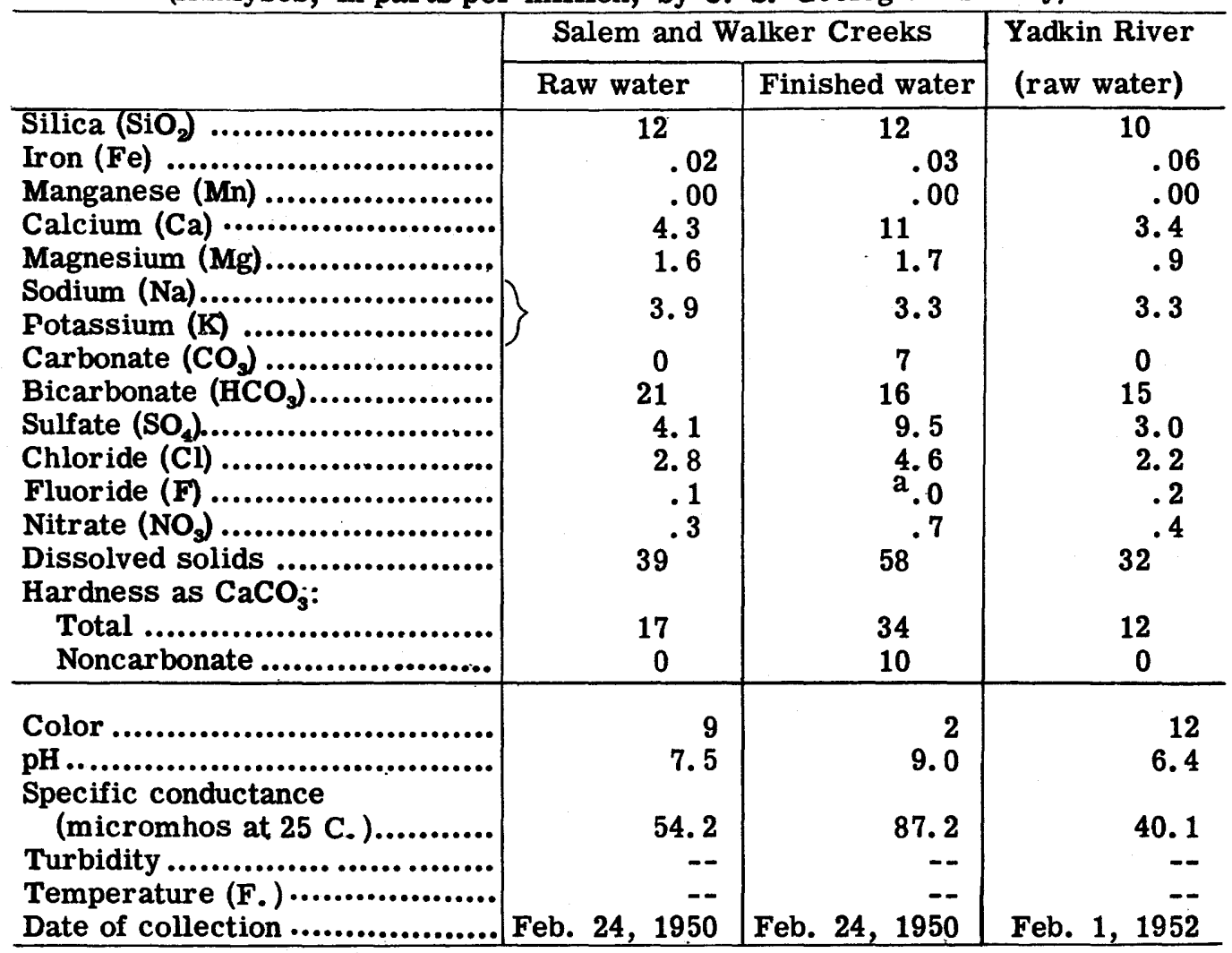

Regular determinations at treatment plant, 1950

\begin{tabular}{|c|c|c|c|c|c|c|c|c|c|c|c|c|}
\hline & \multicolumn{3}{|c|}{$\begin{array}{c}\text { Alkalinity } \\
\text { as } \mathrm{CaCO} \\
\text { (ppm) }\end{array}$} & \multicolumn{3}{|c|}{ pH } & \multicolumn{3}{|c|}{$\begin{array}{l}\text { Hardness } \\
\text { as } \mathrm{CaCO}_{3} \\
\text { (ppm) }\end{array}$} & \multicolumn{3}{|c|}{ Turbidity } \\
\hline & Av & Max & Min & Av & Max & Min & Av & $\operatorname{Max}$ & Min & Av & $\operatorname{Max}$ & Min \\
\hline $\begin{array}{l}\text { Raw water......... } \\
\text { Finished water... }\end{array}$ & $\begin{array}{l}18 \\
25\end{array}$ & \begin{tabular}{|l|}
39 \\
41 \\
\end{tabular} & $\begin{array}{r}8 \\
15\end{array}$ & $\begin{array}{l}7.5 \\
9.2\end{array}$ & \begin{tabular}{|l|}
9.0 \\
9.9
\end{tabular} & $\begin{array}{l}6.8 \\
6.9\end{array}$ & $\begin{array}{l}20 \\
42\end{array}$ & \begin{tabular}{|l|}
34 \\
68
\end{tabular} & \begin{tabular}{|l|}
10 \\
23
\end{tabular} & $\begin{array}{r}17 \\
.09\end{array}$ & \begin{tabular}{|l|}
475 \\
1.7
\end{tabular} & $\begin{array}{r}3 \\
.05\end{array}$ \\
\hline
\end{tabular}

${ }^{a}$ The supply was not being fluoridated at the time of the collection of the sample. Fluoridation was begun later. 


\section{AIKEN \\ (Population, 7, 083)}

Ownership: Municipal; also supplies suburban districts. Total population supplied, about 8,500 .

Source: Springs about 6.5 miles north of the city.

Treatment: Chlorination and lime.

Rated capacity of treatment plant: 2,000,000 gpd.

Raw-water storage: None.

Finished-water storage: Two standpipes, 200,000 and 600,000. gal.

\section{ANALYSES}

(Analyses, in parts per million, by U. S. Geological Survey)

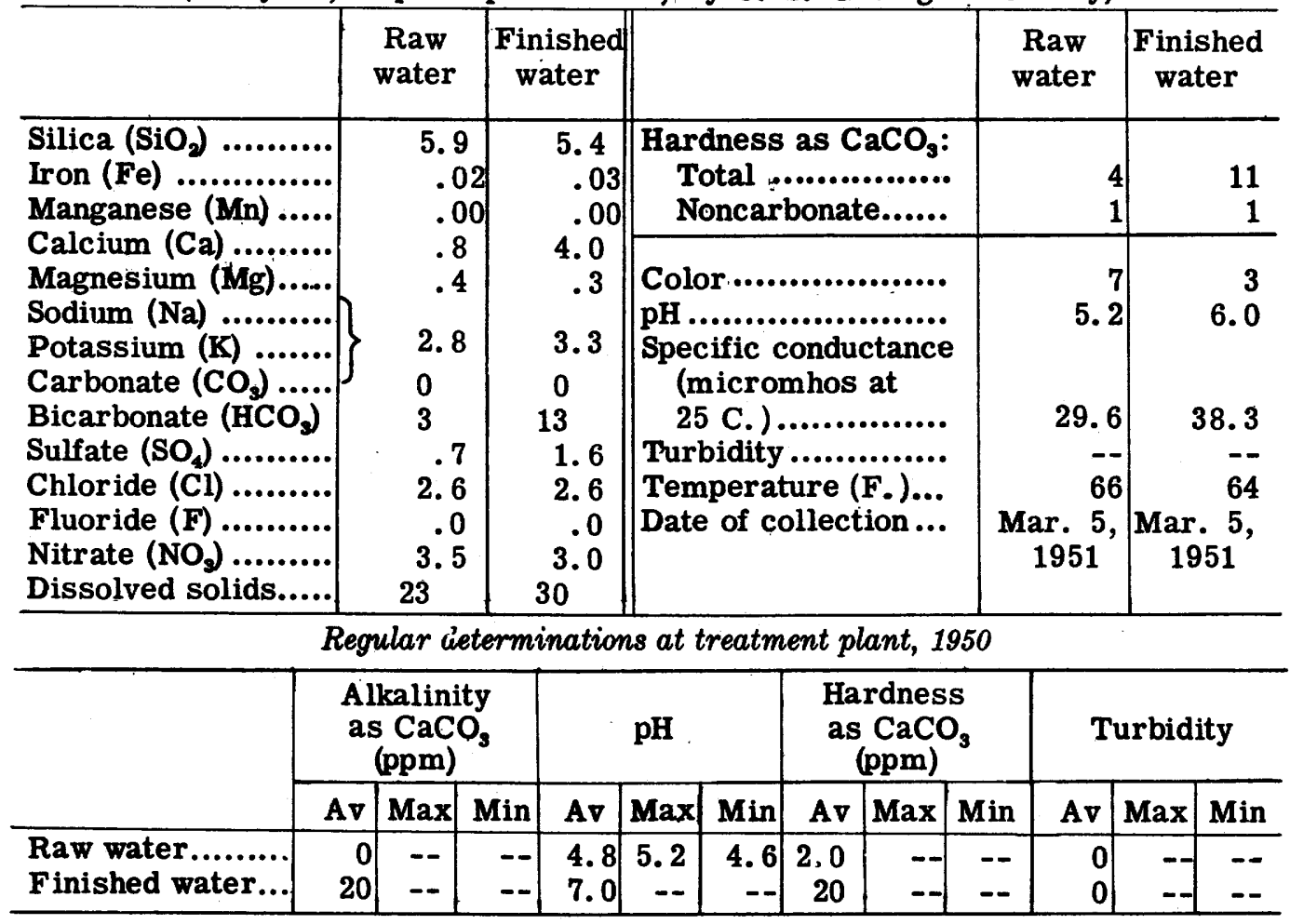




\section{ANDERSON}

(Population, 19, 770)

Ownership: Duke Power Company. Also supplies suburban districts. Total population supplied, about 25,000.

Source: Rocky River, 75 percent of supply; Bailey Creek, 25 percent of supply.

Treatment: Coagulation with alum and lime, sedimentation, rapid sand filtration, chlorination, and adjustment of $\mathrm{pH}$ with soda ash.

Rated capacity of treatment plant: 5, 000,000 gpd.

Raw-water storage: None.

Finished-water storage: 1 clear well, 40,000 gal; 1 standpipe, 198,000 gal; 2 elevated tanks, 500,000 gal each; 2 reservoirs, 1,327,000 and 155, 000 gal.

\section{ANALYSES}

(Analyses, in parts per million, by U. S. Geological Survey)

\begin{tabular}{|c|c|c|c|}
\hline & $\begin{array}{l}\text { Rocky River } \\
\text { Raw water }\end{array}$ & $\begin{array}{c}\text { Bailey Creek } \\
\text { Raw water }\end{array}$ & $\begin{array}{c}\text { Finished water } \\
\text { (city tap) }\end{array}$ \\
\hline 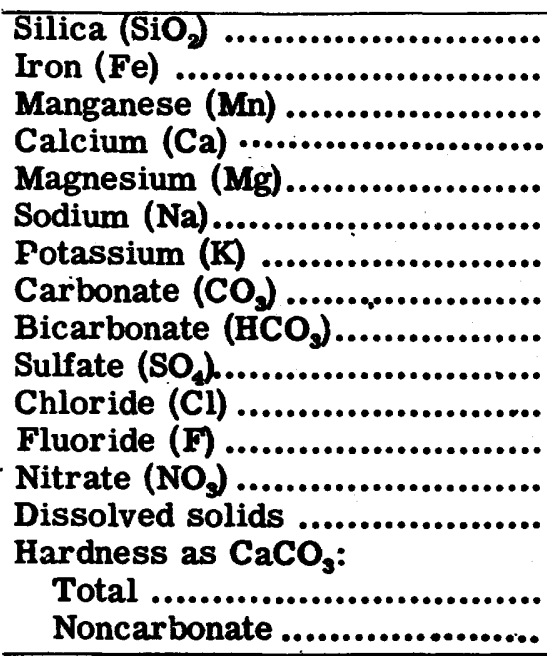 & $\begin{array}{l}17 \\
.11 \\
.00 \\
2.2 \\
.8 \\
8.0 \\
0 \\
22 \\
3.0 \\
2.8 \\
.1 \\
1.0 \\
48 \\
9 \\
0\end{array}$ & $\begin{array}{l}8.5 \\
0 \\
21 \\
5.0 \\
2.5 \\
.1 \\
1.4 \\
46 \\
9 \\
0\end{array}$ & $\begin{array}{l}16 \\
.05 \\
.06 \\
5.8 \\
1.5 \\
8.5 \\
0 \\
27 \\
12 \\
2.4 \\
.1 \\
1.0 \\
62 \\
21 \\
0\end{array}$ \\
\hline 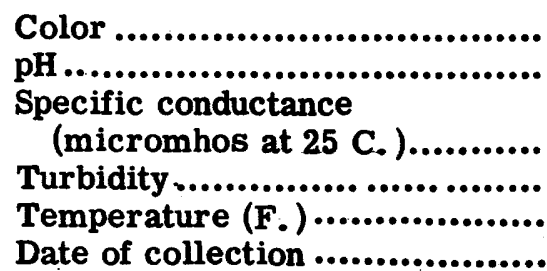 & $\begin{array}{r}27 \\
7.0 \\
53.6 \\
-- \\
51 \\
\text { Feb. } 13,1951\end{array}$ & $\begin{array}{r}6 \\
6.6 \\
52.8 \\
-- \\
52 \\
\text { Feb. } 13,1951\end{array}$ & $\begin{array}{r}7 \\
6.6 \\
84.2 \\
-- \\
45 \\
\text { Feb. } 13,1951\end{array}$ \\
\hline
\end{tabular}

Regular determinations at treatment plant, 1950

\begin{tabular}{|c|c|c|c|c|c|c|c|c|c|c|c|c|}
\hline & \multicolumn{3}{|c|}{$\begin{array}{c}\text { Alkalinity } \\
\text { as } \mathrm{CaCO} \\
\text { (ppm) }\end{array}$} & \multicolumn{3}{|c|}{ pH } & \multicolumn{3}{|c|}{$\begin{array}{c}\text { Hardness } \\
\text { as } \mathrm{CaCO}_{3} \\
\text { (ppm) }\end{array}$} & \multicolumn{3}{|c|}{ Turbidity } \\
\hline & Av & $\operatorname{Max}$ & Min & Av & Max & Min & Av & Max & Min & Av & $\operatorname{Max}$ & Min \\
\hline $\begin{array}{l}\text { Raw water.......... } \\
\text { Finished water... }\end{array}$ & $\begin{array}{l}19 \\
23\end{array}$ & $\begin{array}{l}19 \\
23\end{array}$ & $\begin{array}{l}19 \\
23\end{array}$ & $\begin{array}{l}6.8 \\
7.6\end{array}$ & \begin{tabular}{|l|}
6.9 \\
7.7
\end{tabular} & $\begin{array}{l}6.7 \\
7.5\end{array}$ & $\overline{22}$ & $\overline{22}$ & $\overline{22}$ & $\begin{array}{r}77 \\
0\end{array}$ & $\begin{array}{r}1,500 \\
0\end{array}$ & $\begin{array}{r}25 \\
0\end{array}$ \\
\hline
\end{tabular}




\section{BEAUFORT}

(Population, 5, 081)

Ownership: Municipal; also supplies suburban districts. Total population supplied, about 9,350 .

Source: 5 wells ( 1 to 5) $120,87,65,65$, and $65 \mathrm{ft}$ deep; yield reported to be 350 , $350,250,250$, and $400 \mathrm{gpm}$.

Treatment: Zeolite softening, chlorination at times.

Rated capacity of treatment plant: 1,000,000 gpd.

Raw_water storage: None.

Finished-water storage: 1 elevated tank, 100,000 gal; 1 standpipe, 126,000 gal.

\section{ANALYSIS}

(Analysis, in parts per million, by U. S. Geological Survey)

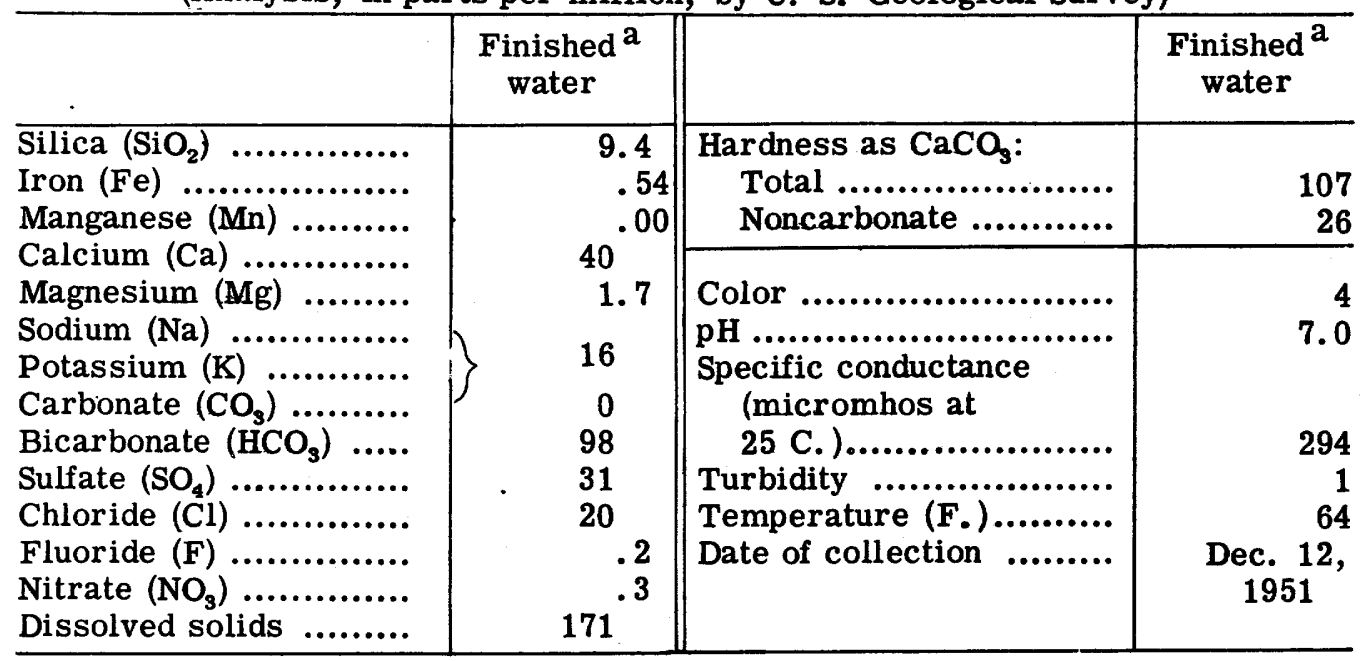

a From wells 2, 3, and 5 . 


\section{BENNETTSVILLE}

(Population, 5, 140)

Ownership: Municipal; supplies also suburban districts. Total population supplied, about 6,000 .

Source: 2 deep wells 300 and $350 \mathrm{ft}$ deep; 1 well field of 8 wells each $60 \mathrm{ft}$ deep; and 1 well field of 5 wells each $60 \mathrm{ft}$ deep.

Treatment: Adjustment of $\mathrm{pH}$ with soda ash.

Rated capacity of treatment plant: 756,000 gpd.

Raw-water storage: None.

Finished-water storage: 1 clear well, 300,000 gal; 1 elevated tank, 100,000 gal.

\section{ANALYSES}

(Analyses, in parts per million, by U. S. Geological Survey)

\begin{tabular}{|c|c|c|c|c|c|}
\hline & $\begin{array}{c}\text { Raw } \\
\text { water }\end{array}$ & $\begin{array}{c}\text { Finished } \\
\text { water }\end{array}$ & & $\begin{array}{c}\text { Raw } \\
\text { water }\end{array}$ & $\begin{array}{l}\text { Finished } \\
\text { water }\end{array}$ \\
\hline $\begin{array}{l}\text { Silica }\left(\mathrm{SiO}_{2}\right) \\
\text { Iron }(\mathrm{Fe}) \ldots \ldots \ldots \ldots \\
\text { Manganese }(\mathrm{Mn}) \ldots \ldots . . . . .\end{array}$ & $\begin{array}{r}10 \\
.06 \\
.00\end{array}$ & $\begin{array}{r}10 \\
.06 \\
.02\end{array}$ & 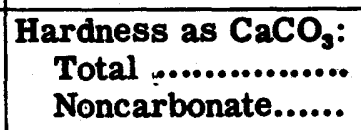 & $\begin{array}{l}9 \\
4\end{array}$ & \\
\hline 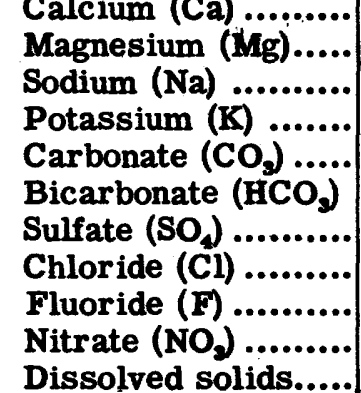 & $\begin{array}{l}1.3 \\
1.4 \\
14 \\
0 \\
6 \\
2.8 \\
14^{.1} \\
15^{.1} \\
64\end{array}$ & $\begin{array}{c}1.6 \\
1.3 \\
28 \\
0 \\
46 \\
1.8 \\
14 \\
.0 \\
14 \\
93\end{array}$ & $\begin{array}{c}\text { Color ................... } \\
\text { pH ......................... } \\
\text { Specific conductance } \\
\text { (micromhos at } \\
25 \text { C. ) ............... } \\
\text { Turbidity ............. } \\
\text { Temperature (F.)... } \\
\text { Date of collection ... }\end{array}$ & $\begin{array}{r}96.9 \\
1 \\
65 \\
\text { Dec. } 17, \\
1951\end{array}$ & $\begin{array}{r}150 \\
1 \\
55 \\
\text { Dec. } 17, \\
1951\end{array}$ \\
\hline
\end{tabular}




\section{CAMDEN}

(Population, 6, 986)

Ownership: Municipal; also supplies suburban districts. Total population supplied, about 8,000.

Source: Little Pine Tree Creek. The intake is about 3 miles east of the city.

Treatment: Prechlorination, coagulation with alum, sedimentation, rapid sand filtration, postchlorination, addition of Calgon, and adjustment of $\mathrm{pH}$ with soda ash.

Rated capacity of treatment plant: 2,000,000 gpd.

Raw-water storage: None.

Finished-water storage: one clear well 65,000 gal; 1 reservoir, 450,000 gal; 1 standpipe, 235,000 gal; 1 elevated tank, 250,000 gal.

\section{ANALYSES}

(Analyses, in parts per million, by U. S. Geological Survey)

\begin{tabular}{|c|c|c|c|c|c|}
\hline & $\begin{array}{l}\text { Raw } \\
\text { water }\end{array}$ & $\left|\begin{array}{c}\text { Finished } \\
\text { water }\end{array}\right|$ & & $\begin{array}{c}\text { Raw } \\
\text { water }\end{array}$ & $\begin{array}{l}\text { Finished } \\
\text { water }\end{array}$ \\
\hline $\begin{array}{l}\text { Silica }\left(\mathrm{SiO}_{2}\right) \ldots \ldots \ldots \ldots . \\
\text { Iron }(\mathrm{Fe}) \ldots \ldots \ldots \ldots . . . . . . . \\
\text { Manganese }(\mathbf{M n}) \ldots . .\end{array}$ & $\begin{array}{c}5.3 \\
.06 \\
.00\end{array}$ & $\begin{array}{l}4.8 \\
.02 \\
.00\end{array}$ & $\begin{array}{r}\text { Hardness as } \mathrm{CaCO}_{3}: \\
\text { Total } \ldots . . . . . . . . . . . . . . \\
\text { Noncarbonate...... }\end{array}$ & $\begin{array}{l}3 \\
0\end{array}$ & \\
\hline $\begin{array}{l}\text { Calcium }(\mathrm{Ca}) . . . . . . . \\
\text { Magnesium }(\mathrm{Mg}) . . . .\end{array}$ & $\begin{array}{l}.6 \\
.3\end{array}$ & $\begin{array}{r}1.0 \\
.3\end{array}$ & |Color ..................... & 45 & \\
\hline $\begin{array}{l}\text { Sodium (Na) ........... } \\
\text { Potassium (K) ....... }\end{array}$ & 2.6 & 14 & $\begin{array}{l}\text { pH ...................................... } \\
\text { Specific conductance }\end{array}$ & 5.4 & 6.2 \\
\hline $\begin{array}{l}\text { Carbonate }\left(\mathrm{CO}_{3}\right) \ldots . . \\
\text { Bicarbonate }\left(\mathrm{HCO}_{3}\right)\end{array}$ & $\begin{array}{l}0 \\
3\end{array}$ & $\begin{array}{r}0 \\
20\end{array}$ & 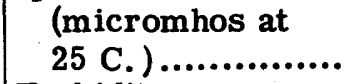 & 21.2 & 73.1 \\
\hline $\begin{array}{l}\text { Sulfate }\left(\mathrm{SO}_{4}\right) \ldots \ldots \ldots \ldots \\
\text { Chloride }(\mathrm{Cl}) \ldots \ldots \ldots\end{array}$ & $\begin{array}{l}2.1 \\
2.2\end{array}$ & $\begin{array}{l}8.9 \\
5.2\end{array}$ & $\begin{array}{l}\text { Turbidity ............... } \\
\text { Temperature (F.)... }\end{array}$ & & -- \\
\hline $\begin{array}{l}\text { Fluoride }(\mathrm{F}) \\
\text { Nitrate }\left(\mathrm{NO} \mathrm{O}_{\mathrm{J}} \ldots \ldots \ldots \ldots \ldots\right. \\
\text { Dissolved solids...... }\end{array}$ & $27^{.4}$ & $45^{.3}$ & Date of collection... & $\underset{1951}{\operatorname{Mar} .8,}$ & $\underset{1951}{\operatorname{Mar} .8,}$ \\
\hline
\end{tabular}

Regular determinations at treatment plant, 1950

\begin{tabular}{l|r|r|r|r|r|r|r|r|r|r|r|r}
\hline & \multicolumn{3}{|c|}{$\begin{array}{c}\text { Alkalinity } \\
\text { as CaCO } \\
\text { (ppm) }\end{array}$} & \multicolumn{3}{c|}{ pH } & \multicolumn{3}{c|}{$\begin{array}{c}\text { Hardness } \\
\text { as CaCO } \\
\text { (ppm) }\end{array}$} & \multicolumn{3}{c}{ Turbidity } \\
\cline { 2 - 12 } & Av & Max & Min & Av & Max & Min & Av & Max & Min & Av & Max & Min \\
\hline Raw water......... & 4 & 5 & 4 & 6.2 & 6 & 5.5 & 4 & 4 & 4 & 65 & 70 &.-- \\
Finished water... & 19 & 22 & 16 & 7 & 7 & 7 & 5 & 5 & 5 & 5 & 5 & 5 \\
\hline
\end{tabular}




\section{CHARLESTON}

(Population, 70, 174)

Ownership: Municipal; also supplies suburban districts. Total population supplied, about 120,000 .

Source: Edisto River 99 percent of supply; Goose Creek impounded in Goose Creek Reservoir 1 percent of supply.

Treatment: Prechlorination, coagulation with alum and sodium aluminate, addition of copper sulfate and activated carbon (Nuchar) occasionally, sedimentation, rapid sand filtration, postchlorination, and addition of caustic soda.

Rated capacity of treatment plant: $22,000,000$ gpd.

Raw-water storage: Goose Creek storage reservoir, 2, 780, 000, 000 gal.

Finished-water storage: 2 ground reservoirs, 2, 000,000 and 350,000 gal; 4 elevated tanks, 2, 000,000,250,000, 200,000, 75, 000 gal.

Water from the Edisto River flows by gravity through a tunnel aqueduct direct to pumping station or to Goose Creek as desired.

\section{ANALYSES}

(Analyses, in parts per million, by U. S. Geological Survey)

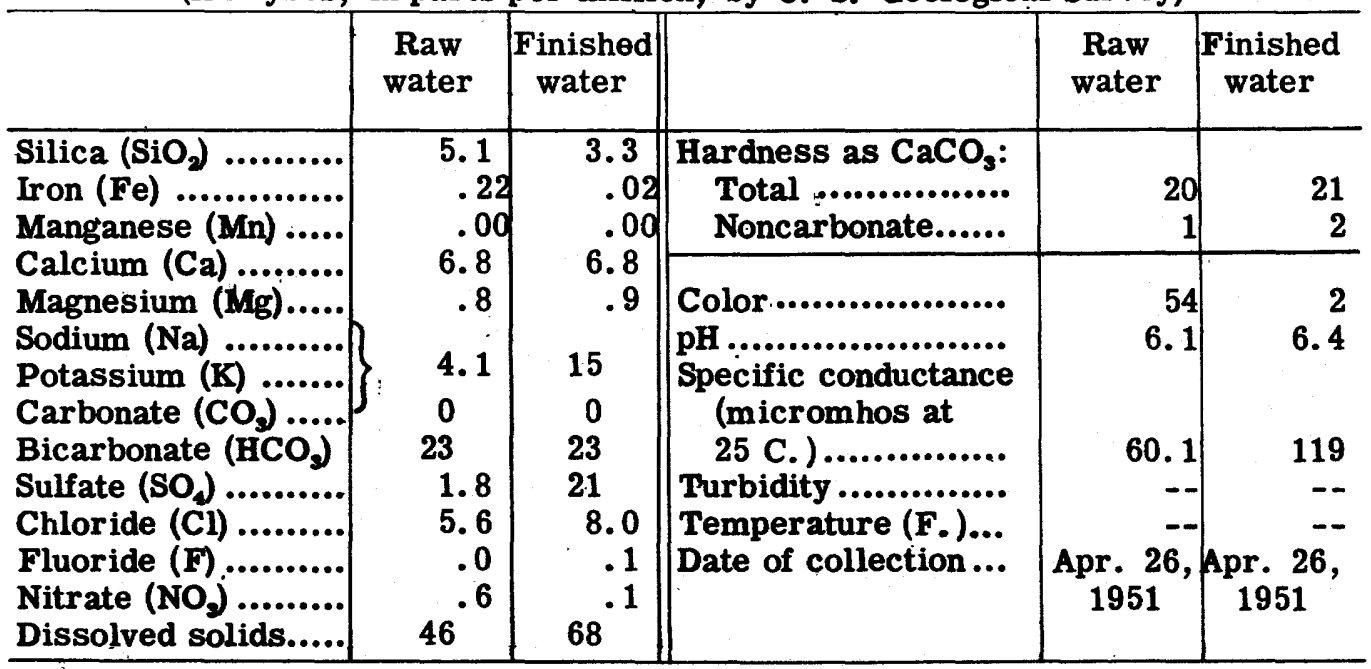

Regular determinations at treatment plant, 1950

\begin{tabular}{l|r|r|r|r|r|r|r|r|r|r|r|r}
\hline & \multicolumn{3}{|c|}{$\begin{array}{c}\text { Alkalinity } \\
\text { as CaCO, } \\
\text { (ppm) }\end{array}$} & \multicolumn{3}{c|}{ pH } & \multicolumn{3}{c|}{$\begin{array}{c}\text { Hardness } \\
\text { as CaCO, } \\
\text { (ppm) }\end{array}$} & \multicolumn{3}{c}{ Turbidity } \\
\cline { 2 - 13 } & Av & Max & Min & Av & Max & Min & Av & Max & Min & Av & Max & Min \\
\hline Raw water......... & 10 & 17 & 5 & 6.6 & 7.1 & 6.0 & -- & -- & - & 81 & 180 & 50 \\
Finished water... & 17 & 27 & 12 & $\mathbf{8 . 4}$ & $\mathbf{9 . 2}$ & 7.6 & -- & -- & -- & 6 & 30 & 2 \\
\hline
\end{tabular}




\section{CHEST ER}

(Population, 6, 893)

Ownership: Municipal; also supplies suburban districts. Total population supplied, about 8,000.

Source: Sandy River impounded. The intake is about 2.5 miles west of the city.

Treatment: Prechlorination, coagulation with alum, sedimentation, rapid sand filtration, and adjustment of $\mathrm{pH}$ with soda ash.

Rated capacity of treatment plant: 2, 500,000 gpd.

Raw-water storage: Impounding reservoir, 534,000,000 gal.

Finished-water storage: 2 reservoirs, 500,000, 267,000 gal; 2 elevated tanks, 250,000 and 150,000 gal.

\section{ANALYSES}

(Analyses, in parts per million, by U. S. Geological Survey)

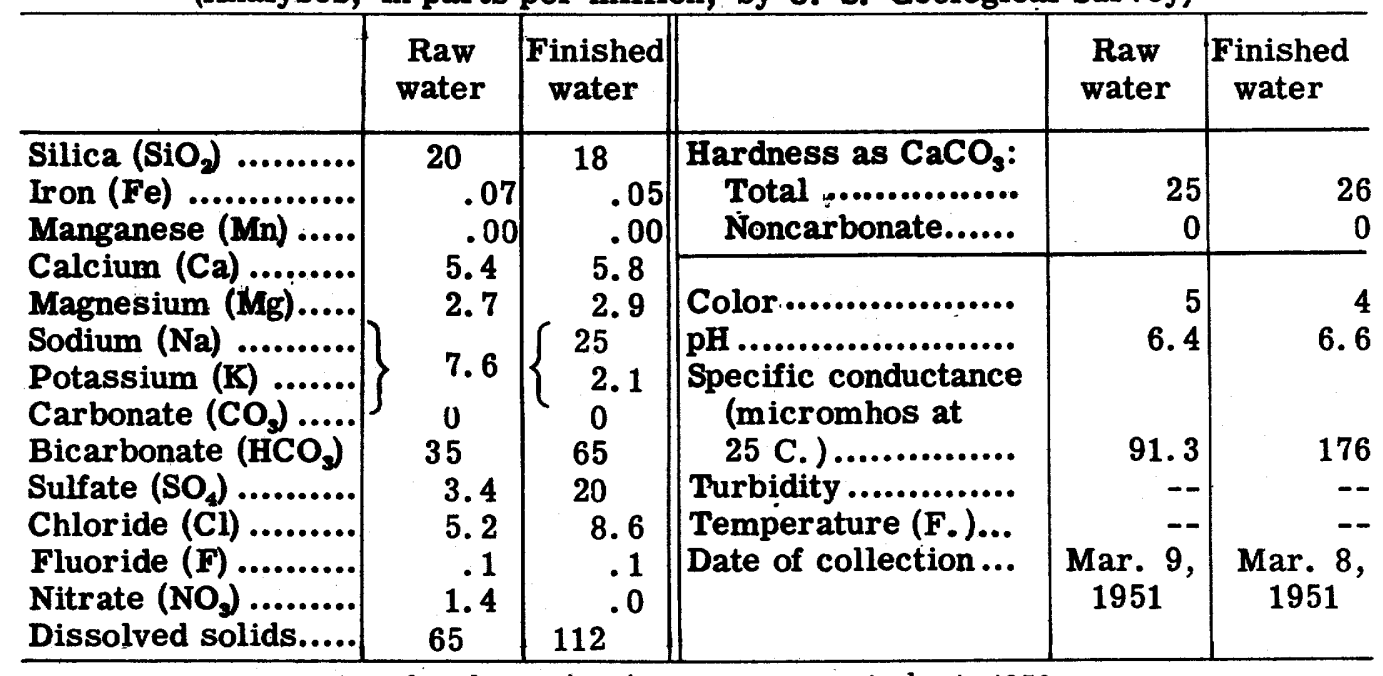

Regular determinations at treatment plant, 1950

\begin{tabular}{l|r|r|r|r|r|r|r|r|r|r|r|r}
\hline & \multicolumn{3}{|c|}{$\begin{array}{c}\text { Alkalinity } \\
\text { as CaCO, } \\
\text { (ppm) }\end{array}$} & \multicolumn{3}{c|}{ pH } & \multicolumn{3}{c|}{$\begin{array}{c}\text { Hardness } \\
\text { as CaCO, } \\
\text { (ppm) }\end{array}$} & \multicolumn{3}{c}{ Turbidity } \\
\cline { 2 - 13 } & Av & Max & Min & Av & Max & Min & Av & Max & Min & Av & Max & Min \\
\hline Raw water......... & 24 & 32 & 17 & 6.8 & 7.4 & 6.0 & -- & -- & -- & 150 & 4,000 & 100 \\
Finished water... & 46 & 42 & 40 & 8.4 & 8.4 & 8.4 & 12 & 16 & 10 & 0 & 0 & 0 \\
\hline
\end{tabular}




\section{CLINTON \\ (Population, 7, 168)}

Ownership: Municipal; also supplies suburban districts. Total population supplied, about 4,580. Private systems supply the remaining population of the city.

Source: Duncan Creek. The intake is about 5 miles northeast of the city.

Treatment: Prechlorination, coagulation with alum and lime, sedimentation, rapid sand filtration, and adjustment of pH with soda ash.

Rated capacity of treatment plant: $1,000,000 \mathrm{gpd}$.

Raw-water storage: 2 reservoirs, 600,000 and 625,000 gal.

Finished-water storage: 1 clear well, 250,000 gal; 2 elevated tanks, 250,000 and 100,000 gal.

\section{ANALYSES}

(Analyses, in parts per million, by U. S. Geological Survey)

\begin{tabular}{|c|c|c|c|c|c|}
\hline & $\begin{array}{c}\text { Raw } \\
\text { water }\end{array}$ & $\begin{array}{c}\text { Finished } \\
\text { water }\end{array}$ & & $\begin{array}{c}\text { Raw } \\
\text { water }\end{array}$ & $\begin{array}{c}\text { Finished } \\
\text { water }\end{array}$ \\
\hline 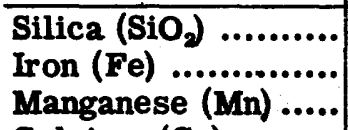 & $\begin{array}{l}23 \\
.05 \\
.00\end{array}$ & $\begin{array}{l}23 \\
.03 \\
.00\end{array}$ & 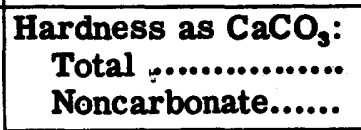 & $\begin{array}{r}16 \\
0\end{array}$ & \\
\hline 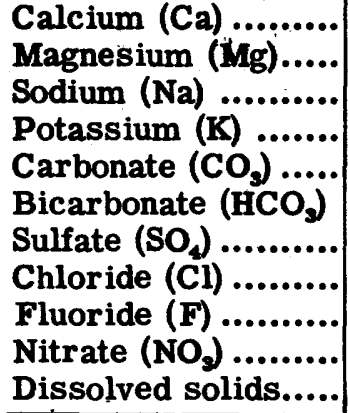 & $\begin{array}{c}4.1 \\
1.5 \\
6.0 \\
0 \\
25 \\
3.0 \\
3.4 \\
.2 \\
.5\end{array}$ & $\begin{array}{r}5.6 \\
1.6 \\
14 \\
0 \\
37 \\
15 \\
3.9 \\
.1 \\
.3\end{array}$ & $\begin{array}{c}\text { Color................... } \\
\text { pH ......................... } \\
\text { Specific conductance } \\
\text { (micromhos at } \\
25 \text { C.) ............... } \\
\text { Turbidity ............. } \\
\text { Temperature (F.)... } \\
\text { Date of collection ... }\end{array}$ & $\begin{array}{r}61.2 \\
24 \\
43 \\
\text { Dec. } 20, \\
1951\end{array}$ & \begin{tabular}{|c}
114 \\
1 \\
43 \\
Dec. 20 \\
1951
\end{tabular} \\
\hline
\end{tabular}




\section{COLUMBIA}

(Population, 86, 914)

Ownership: Municipal; also supplies suburban districts. Total population supplied, about 102,000 .

Source: Congaree River.

Treatment: Coagulation with alum and lime, prechlorination (breakpoint), sedimentation, rapid sand filtration, and adjustment of $\mathrm{pH}$ with lime.

Rated capacity of treatment plant: $18,000,000$ gpd.

Raw-water storage: 40,000,000 gal.

Finished-water storage: 2 clear water reservoirs, 2, 800, 000 and 3, 000, 000 gal; 1 standpipe, 375, $000 \mathrm{gal}$; 1 elevated tank, 1, 500,000 gal.

\section{ANALYSES}

(Analyses, in parts per million, by U. S. Geological Survey)

\begin{tabular}{|c|c|c|c|c|c|}
\hline & $\begin{array}{l}\text { Raw } \\
\text { water }\end{array}$ & $\begin{array}{l}\text { Finished } \\
\text { water }\end{array}$ & & $\begin{array}{l}\text { Raw } \\
\text { water }\end{array}$ & $\begin{array}{c}\text { Finished } \\
\text { water }\end{array}$ \\
\hline $\begin{array}{l}\text { Silica }\left(\mathrm{SiO}_{2}\right) \\
\text { Iron }(\mathrm{Fe}) \ldots \ldots \ldots \ldots . . . . . . . . . \\
\text { Manganese }(\mathrm{Mn}) \ldots . .\end{array}$ & $\begin{array}{r}14 \\
.03 \\
.00\end{array}$ & $\begin{array}{r}12 \\
.03 \\
.00\end{array}$ & $\begin{array}{c}\text { Hardness as } \mathrm{CaCO}_{3} \\
\text { Total } \\
\text { Noncarbonate....................... }\end{array}$ & $\begin{array}{r}22 \\
0\end{array}$ & $\begin{array}{r}29 \\
3\end{array}$ \\
\hline $\begin{array}{l}\text { Calcium (Ca) .......... } \\
\text { Magnesium (Mg).... }\end{array}$ & $\begin{array}{l}6.0 \\
1.6\end{array}$ & $\begin{array}{l}8.5 \\
1.9\end{array}$ & Color....................... & 2 & 3 \\
\hline $\begin{array}{l}\text { Sodium (Na) ..... } \\
\text { Potassium (K) .. }\end{array}$ & 7.7 & 6.9 & 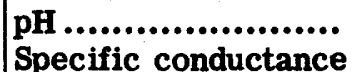 & 6.5 & 6.6 \\
\hline Carbonate $\left(\mathrm{CO}_{3}\right) \ldots . . .$. & $\begin{array}{r}0 \\
34\end{array}$ & $\begin{array}{r}0 \\
32\end{array}$ & 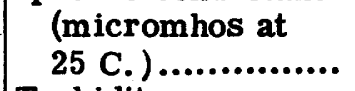 & 71.5 & 97.1 \\
\hline $\begin{array}{l}\text { Sulfate }\left(\mathrm{SO}_{\mathcal{L}}\right) \ldots \ldots \ldots \\
\text { Chloride }(\mathrm{Cl}) \ldots \ldots \ldots\end{array}$ & 4.0 & 10 & Turbidity ............... & -- & -- \\
\hline $\begin{array}{l}\text { Fluoride }(F) \ldots . . . . . . . \\
\text { Nitrate }\left(\mathrm{NO}_{3}\right) \ldots . . . . . \\
\text { Dissolved solids..... }\end{array}$ & $\begin{aligned} .1 \\
.5\end{aligned}$ & $63^{.3}$ & Date of collection... & $\underset{1951}{\operatorname{Mar} .9,}$ & $\underset{1951}{\operatorname{Mar} .9}$ \\
\hline
\end{tabular}

Regular determinations at treatment plant, 1950

\begin{tabular}{l|r|r|r|r|r|r|r|r|r|r|r|r}
\hline & \multicolumn{3}{|c|}{$\begin{array}{c}\text { Alkalinity } \\
\text { as CaCO } \\
\text { (ppm) }\end{array}$} & \multicolumn{3}{c|}{ pH } & \multicolumn{3}{c|}{$\begin{array}{c}\text { Hardness } \\
\text { as CaCO } \\
\text { (ppm) }\end{array}$} & \multicolumn{3}{c}{ Turbidity } \\
\cline { 2 - 14 } & Av & Max & Min & Av & Max & Min & Av & Max & Min & Av & Max & Min \\
\hline Raw water........ & 23 & 28 & 16 & 7.0 & 7.3 & 6.8 & -- & -- & -- & 75 & 370 & 25 \\
Finished water... & 24 & 29 & 18 & 8.4 & 8.9 & 7.2 & 33 & 42 & 24 & -- & -- & -- \\
\hline
\end{tabular}


CONWAY

(Population, 6, 073)

Ownership: Municipal; also supplies suburban districts. Total population supplied, about 8,000 .

Source: 10 wells: Park Well and Hill Well, 438 and $406 \mathrm{ft}$ deep; 3 artesian wells at plant $400 \mathrm{ft}$ deep; 3 artesian wells in cemetery, $210 \mathrm{ft}$ deep; 2 artesian wells on 1st Avenue, 290 and $286 \mathrm{ft}$ deep. The wells are flowing except the first two named.

Treatment: None.

Rated capacity of plant: $1,000,000$ gpd.

Storage: Reservoirs, 400, 000 gal; 1 elevated tank, 100,000 gal.

\section{ANALYSES}

(Analyses, in parts per million, by U. S. Geological Survey)

\begin{tabular}{|c|c|c|c|}
\hline & Park Well & Hill Well & $\begin{array}{l}3 \text { wells } \\
\text { at plant }\end{array}$ \\
\hline 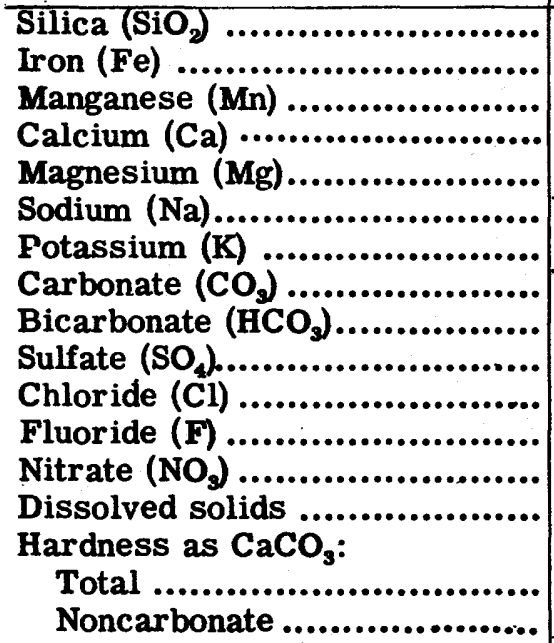 & $\begin{array}{l}15 \\
.05 \\
.00 \\
2.6 \\
.8 \\
264^{.8} \\
22 \\
540 \\
67^{.9} \\
3.5 \\
647^{.5} \\
10 \\
0\end{array}$ & $\begin{array}{l}12 \\
.16 \\
.00 \\
2.8 \\
1.3 \\
245 \\
14 \\
568 \\
1.2 \\
31 \\
4.2 \\
601 \\
.4 \\
12 \\
0\end{array}$ & $\begin{array}{c}15 \\
.04 \\
.00 \\
2.3 \\
1.1 \\
254 \\
24 \\
542 \\
1.0 \\
47 \\
3.8 \\
.4 \\
620 \\
10 \\
0\end{array}$ \\
\hline 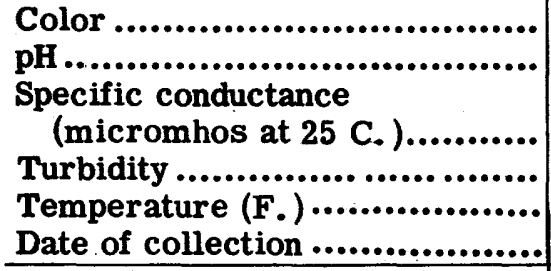 & $\begin{array}{r}13 \\
8.2 \\
1,070 \\
1 \\
69 \\
\text { Dec: } 19,1951 \\
\end{array}$ & $\begin{array}{r}12 \\
8.1 \\
974 \\
1 \\
69 \\
\text { Dec. } 19,1951 \\
\end{array}$ & $\begin{array}{r}14 \\
8.3 \\
1,010 \\
1 \\
60 \\
\text { Dec. } 19,1951 \\
\end{array}$ \\
\hline 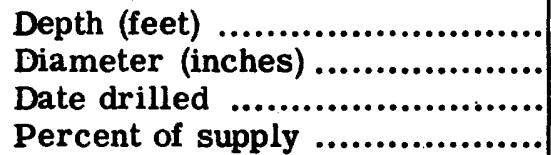 & $\begin{array}{r}438 \\
8 \\
1936 \\
--\end{array}$ & $\begin{array}{r}406 \\
8 \\
1946 \\
--\end{array}$ & $\begin{array}{r}400 \\
10 \\
1918 \\
--\end{array}$ \\
\hline
\end{tabular}




\section{DARLINGTON}

(Population, 6, 619)

Ownership: Municipal; also supplies suburban districts. Total population supplied, about 7,600.

Source: 4 wells (1 to 4) $315,317,570$, and $450 \mathrm{ft}$ deep; yield reported to be 300 , $400,100,530 \mathrm{gpm}$. Well 4 is for emergency use.

Treatment: Adjustment of $\mathrm{pH}$ with lime.

Rated capacity of treatment plant: 800,000 gpd.

Raw-water storage: None.

Finished-water storage: Reservoir, 300,000 gal; 1 elevated tank, 90,000 gal.

\section{ANALYSES}

(Analyses, in parts per million, by U. S. Geological Survey)

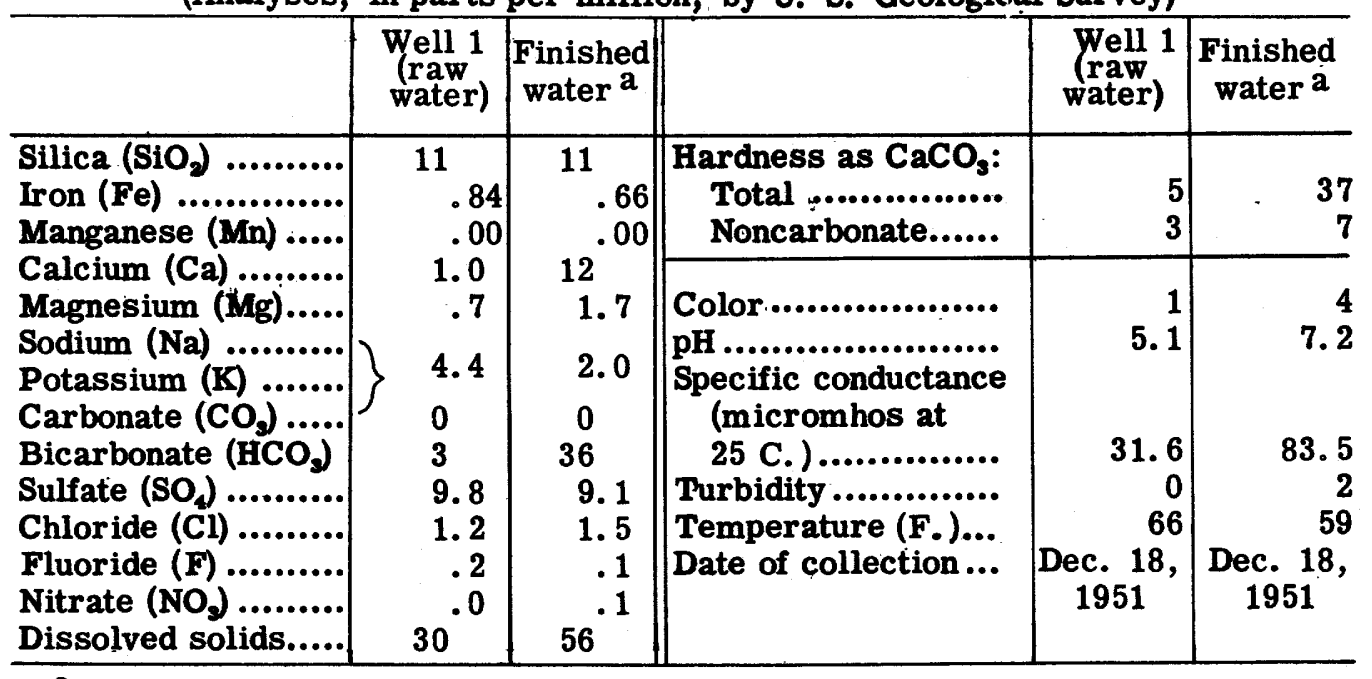

${ }^{a}$ Wells 1 and 2. 
EASLEY

(Population, 6, 316)

Ownership: Municipal; also supplies suburban districts. Total population supplied, about 8, 400 .

Source: Burdine Creek (70 percent of supply); Maddog Branch (30 percent of supply). The intake on Burdine Creek is 3 miles northeast of the city; on Maddog Branch, about 1 mile northeast of the city.

Treatment: Prechlorination, coagulation with alum and lime, sedimentation, rapid sand filtration, adjustment of $\mathrm{pH}$ with soda ash, (sodium metaphosphate and ammonia occasionally).

Rated capacity of treatment plant: $2,000,000$ gpd.

Raw-water storage: None.

Finished-water storage: Clear well, 900,000 gal; 3 elevated tanks, 500,000, 200,000 , and 60,000 gal.

\section{ANALYSES}

(Analyses, in parts per million, by U. S. Geological Survey)

\begin{tabular}{|c|c|c|c|c|c|}
\hline & $\begin{array}{l}\text { Raw } \\
\text { water }\end{array}$ & $\begin{array}{c}\text { Finished } \\
\text { water. }\end{array}$ & & $\begin{array}{c}\text { Raw } \\
\text { water }\end{array}$ & $\begin{array}{l}\text { Finished } \\
\text { water }\end{array}$ \\
\hline 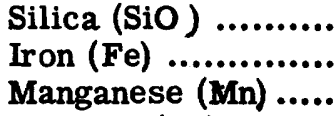 & \multirow{2}{*}{$\begin{array}{r}11 \\
.06 \\
.00 \\
2.8 \\
.7\end{array}$} & \multirow{2}{*}{$\begin{array}{r}10 \\
.04 \\
.00 \\
4.4 \\
.7\end{array}$} & $\begin{array}{r}\text { Hardness as } \mathrm{CaCO}_{3} \text { : } \\
\text { Total ................... } \\
\text { Noncarbonate..... }\end{array}$ & $\begin{array}{r}10 \\
2\end{array}$ & $\begin{array}{r}14 \\
0\end{array}$ \\
\hline $\begin{array}{l}\text { Calcium (Ca) ......... } \\
\text { Magnesium (Mg)..... }\end{array}$ & & & Color ................. & 5 & \\
\hline Sodium (Na) ........... & 2.3 & 7.3 & pH ......................... & 6.8 & 7.2 \\
\hline Carbonate $\left(\mathrm{CO}_{3}\right) \ldots$. & 0 & 0 & (micromhos at & & \\
\hline Bicarbonate $\left(\mathrm{HCO}_{2}\right)$ & 10 & 18 & 25 C. ) ................ & 31.2 & 65.9 \\
\hline Sulfate $\left(\mathrm{SO}_{4}\right) \ldots \ldots \ldots$ & 2.0 & 8.1 & Turbidity ............... & 30 & \\
\hline de $(\mathrm{Cl}) \ldots \ldots \ldots$ & 2.1 & 3.5 & Temperature (F.)... & 44 & 46 \\
\hline Fluoride (F) ........... & & .2 & Date of collection... & Dec. 20, & Dec. 20, \\
\hline$e\left(\mathrm{NO}_{3}\right) \ldots \ldots \ldots$ & 1.5 & 1.3 & & 1951 & 1951 \\
\hline Dissolved solids..... & 28 & & & & \\
\hline
\end{tabular}

Regular determinations at treatment plant, 1950

\begin{tabular}{l|r|r|r|r|r|r|r|r|r|r|r|r}
\hline & \multicolumn{3}{|c|}{$\begin{array}{c}\text { Alkalinity } \\
\text { as CaCO } \\
\text { (ppm) }\end{array}$} & \multicolumn{3}{c|}{ pH } & \multicolumn{3}{c|}{$\begin{array}{c}\text { Hardness } \\
\text { as CaCO } \\
\text { (ppm) }\end{array}$} & \multicolumn{3}{c}{ Turbidity } \\
\cline { 2 - 13 } & Av & Max & Min & Av & Max & Min & Av & Max & Min & Av & Max & Min \\
\hline Raw water......... & 14 & 17 & 10 & 6.3 & 6.9 & 5.7 & -- & -- & -- & 301,000 & 25 \\
Finished water... & 22 & 27 & -- & 7.3 & 7.4 & 7 & 16 & 20 & 14 & 5 & 5 \\
\hline
\end{tabular}


FLORENCE

(Population, 22, 513)

Ownership: Municipal; also supplies suburban areas. Total population supplied, about 25, 000 .

Source: 5 wells $(6,9$ to 12$) 735,728,768,740$, and $758 \mathrm{ft}$ deep; yield reported to be $860,300,740,820$, and $920 \mathrm{gpm}$.

Treatment: Aeration, filtration (iron and $\mathrm{CO}_{2}$ removal), $\mathrm{pH}$ adjustment, and chlorination.

Rated capacity of treatment plant: 4,896,000 gpd.

Raw-water storage: None.

Finished-water storage: 2 ground reservoirs, 1,400,000 gal; 3 elevated tanks, $100,000,500,000$, and 300,000 gal.

\section{ANALYSES}

(Analyses, in parts per million, by U. S. Geological Survey)

\begin{tabular}{|c|c|c|c|}
\hline & $\begin{array}{c}\text { Well } 6 \\
\text { (raw water) }\end{array}$ & $\begin{array}{c}\text { Well } 12 \\
\text { (raw water) }\end{array}$ & $\begin{array}{c}\text { Wells } 6,9, \\
10,11 \\
\text { (finished water) }\end{array}$ \\
\hline 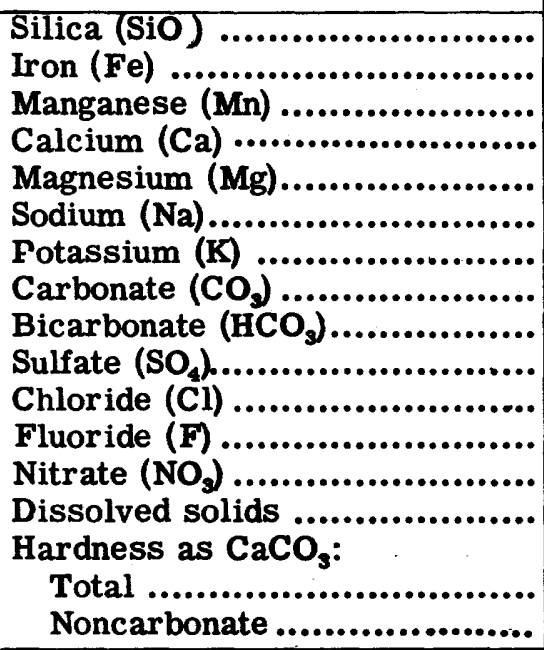 & $\begin{array}{c}31 \\
3.4 \\
.00 \\
7.6 \\
2.6 \\
12 \\
0 \\
19 \\
9.8 \\
20 \\
.3 \\
.0 \\
96 \\
30 \\
14 \\
\end{array}$ & $\begin{array}{c}18 \\
1.2 \\
.00 \\
2.1 \\
1.5 \\
17 \\
0 \\
27 \\
8.5 \\
12 \\
.1 \\
.0 \\
71 \\
11 \\
0\end{array}$ & $\begin{array}{r}0 \\
85 \\
10 \\
20 \\
.2 \\
.1 \\
140 \\
\\
41 \\
0\end{array}$ \\
\hline 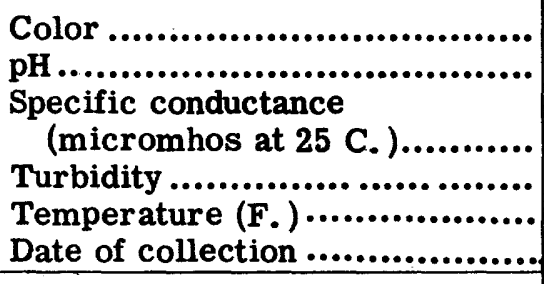 & $\begin{array}{r}3 \\
5.8 \\
129 \\
0 \\
67 \\
\text { Dec. } 18,1951 \\
\end{array}$ & $\begin{array}{r}6.2 \\
106 \\
0 \\
69 \\
\text { Dec. } 18,1951 \\
\end{array}$ & $\begin{array}{r}7.2 \\
72 \\
228 \\
0 \\
69 \\
\text { Dec. } 18,1951 \\
\end{array}$ \\
\hline 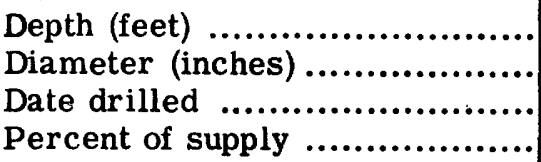 & $\begin{array}{r}735 \\
18 \\
\text { Nov., } 1930 \\
--\end{array}$ & $\begin{array}{r}785 \\
20 \\
\text { July, } 1950\end{array}$ & \\
\hline
\end{tabular}




\section{GAF FNEY}

(Population, 8, 123)

Ownership: Municipal; also supplies suburban districts. Total population supplied, about 11,000 .

Source: Cherokee Creek.

Treatment: Coagulation with alum and lime, activated carbon, sedimentation, rapid sand filtration, chlorination, Calgon, and adjustment of $\mathrm{pH}$ with lime.

Rated capacity of treatment plant: 2,000,000 gpd.

Raw-water storage: 2,000,000 gal.

Finished-water storage: 2 clear wells, 450,000 and 100,000 gal; 1 standpipe, 500,000 gal; 1 elevated tank, 500,000 gal.

The treatment plant is on the creek 3.5 miles from the city.

\section{ANALYSES}

(Analyses, in parts per million, by U. S. Geological Survey)

\begin{tabular}{|c|c|c|c|c|c|}
\hline & $\begin{array}{r}\text { Raw } \\
\text { water }\end{array}$ & $\begin{array}{c}\text { Finished } \\
\text { water }\end{array}$ & & $\begin{array}{l}\text { Raw } \\
\text { water }\end{array}$ & $\begin{array}{l}\text { Finished } \\
\text { water }\end{array}$ \\
\hline \multirow{8}{*}{ 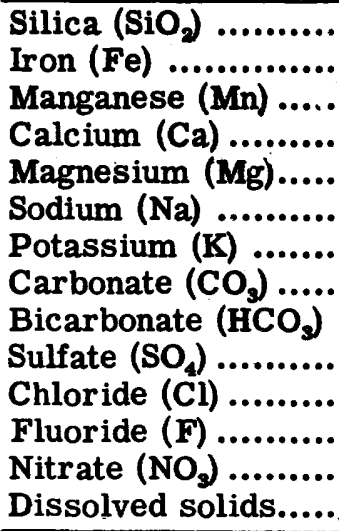 } & \multirow{2}{*}{$\begin{array}{r}11 \\
.07 \\
.0 \\
2.4 \\
1.2\end{array}$} & \multirow{2}{*}{$\begin{array}{c}10 \\
.04 \\
.07 \\
4.0 \\
1.2\end{array}$} & $\begin{array}{r}\text { Hardness as } \mathrm{CaCO}_{\mathbf{3}} \text { : } \\
\text { Total ................... } \\
\text { Noncarbonate..... }\end{array}$ & $\begin{array}{r}11 \\
0\end{array}$ & $\begin{array}{r}15 \\
4 \\
\end{array}$ \\
\hline & & & $\begin{array}{l}\text { Color } \ldots \ldots \ldots \ldots \ldots \ldots \ldots \\
\text { pH } \ldots \ldots \ldots \ldots \ldots \ldots \ldots \ldots\end{array}$ & $\begin{array}{r}7 \\
6.0\end{array}$ & $\begin{array}{r}6 \\
6.3\end{array}$ \\
\hline & 4.1 & 3.4 & Specific conductance & & \\
\hline & & & & 41.7 & 52.4 \\
\hline & 2.4 & 5.8 & Turbidity ................ & -- & -- \\
\hline & 3.0 & 3.1 & Temperature (F.)... & & \\
\hline & .0 & .0 & Date of collection... & Feb. 14, & Feb. 14, \\
\hline & $33^{1.0}$ & $37^{.7}$ & & & \\
\hline
\end{tabular}

Regular determinations at treatment plant, 1950

\begin{tabular}{l|r|r|r|r|r|r|r|r|r|r|r|r}
\hline & \multicolumn{2}{|c|}{$\begin{array}{c}\text { Alkalinity } \\
\text { as CaCO, } \\
\text { (ppm) }\end{array}$} & \multicolumn{3}{c|}{ pH } & \multicolumn{3}{c|}{$\begin{array}{c}\text { Hardness } \\
\text { as CaCO } \\
\text { (ppm) }\end{array}$} & \multicolumn{3}{c}{ Turbidity } \\
\cline { 2 - 13 } & Av & Max & Min & Av & Max & Min & Av & Max & Min & Av & Max & Min \\
\hline Raw water......... & 9 & 10 & 7 & 6.7 & 7.1 & 6.5 & -- & - & -- & 75 & 375 & 30 \\
Finished water... & 12 & 14 & 10 & 7.3 & 7.5 & 7.1 & 21 & 26 & 18 & -- & - & -- \\
\hline
\end{tabular}


GEORGETOWN

(Population, 6, 004)

Ownership: Municipal; also supplies suburban districts. Total population supplied, about 8,500.

Source: 5 wells ( 1 to 5 ) $650,850,850,925$, and $800 \mathrm{ft}$ deep; yield reported to be $190,210,150,200$, and $400 \mathrm{gpm}$.

Treatment: Chlorination.

Raw-water storage: A reservoir, 230,000 gal.

Finished-water storage: 1 standpipe, 146,000 gal.

Water from well 1 and 2 is pumped into a reservoir and is chlorinated when pumped from this to the distribution system.

ANALYSES

(Analyses, in parts per million, by U. S. Geological Survey)

\begin{tabular}{|c|c|c|c|}
\hline & $\begin{array}{l}\text { Wells } 1 \text { and } 2 \\
\text { (raw water) }\end{array}$ & $\begin{array}{c}\text { Well } 3 \\
\text { (raw water) }\end{array}$ & $\begin{array}{c}\text { Well } 4 \\
\text { (raw water) }\end{array}$ \\
\hline 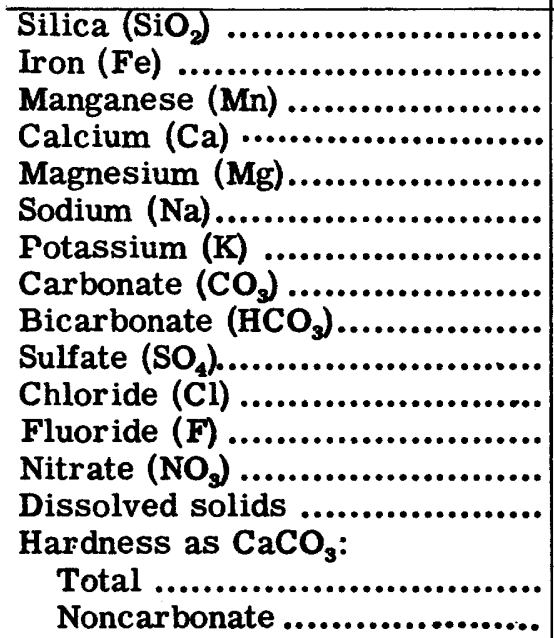 & $\begin{array}{l}13 \\
.13 \\
.00 \\
2.1 \\
.6 \\
215 \\
26 \\
467 \\
1.4 \\
32 \\
.9 \\
1.1 \\
522 \\
8 \\
0\end{array}$ & $\begin{array}{l}12 \\
.17 \\
.00 \\
2.0 \\
.9 \\
219 \\
20 \\
463 \\
3.0 \\
46 \\
1.1 \\
.8 \\
536 \\
9 \\
0\end{array}$ & $\begin{array}{c}14 \\
.12 \\
.00 \\
2.4 \\
.7 \\
246 \\
17 \\
528 \\
2.1 \\
54 \\
1.1 \\
.8 \\
599 \\
9 \\
0\end{array}$ \\
\hline 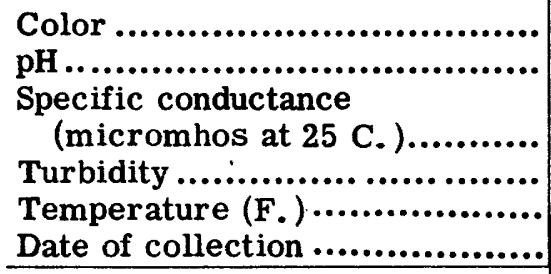 & $\begin{array}{r}5 \\
8.5 \\
356 \\
0 \\
-- \\
\text { Dec. } 19,1951 \\
\end{array}$ & \begin{tabular}{|r}
4 \\
8.3 \\
878 \\
1 \\
76 \\
Dec. 19,1951 \\
\end{tabular} & \begin{tabular}{|r|}
3 \\
8.4 \\
982 \\
1 \\
-- \\
Dec. 19,1951 \\
\end{tabular} \\
\hline 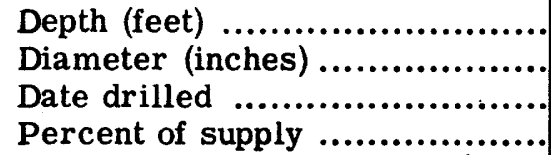 & $\begin{array}{r}650 \\
8 \\
1899 \\
--\end{array}$ & $\begin{array}{r}850 \\
8 \\
1932 \\
--\end{array}$ & $\begin{array}{r}925 \\
8 \\
1947 \\
--\end{array}$ \\
\hline
\end{tabular}




\section{GREENVILLE}

(Population, 58, 161)

Ownership: Municipal; also supplies suburban districts. Total population supplied, about 135,000 .

Source: Mountain streams, impounded.

Treatment: Chlorination, ammoniation, and soda-ash at Plant 1. Additional chlorination and ammoniation at Plants 2 and 3.

Rated capacity of treatment plant: 40,000,000 gpd.

Raw-water storage: Impounding reservoir about 9, 500, 000, 000 gal.

Finished-water storage: 1 reservoir, 22,000,000 gal; 2 standpipes, 1, 220,000 and 460,000 gal.

Table Rock Reservoir is about 22.8 miles from the city limits. Plant 1 is about 1,000 feet from the reservoir. Water from Table Rock reservoir is treated at Plant 1, flows to two equalizing reservoirs, No. 3, 19.3 miles east of Table Rock Reservoir and No. 6, 16.7 miles southeast of Table Rock Reservoir. From these reservoirs, water flows through two separate lines to chemical plants 2 and 3 for additional treatment with ammonia and chlorine before entering the distribution system and standpipes.

\section{ANALYSES}

(Analyses, in parts per million, by U. S. Geological Survey)

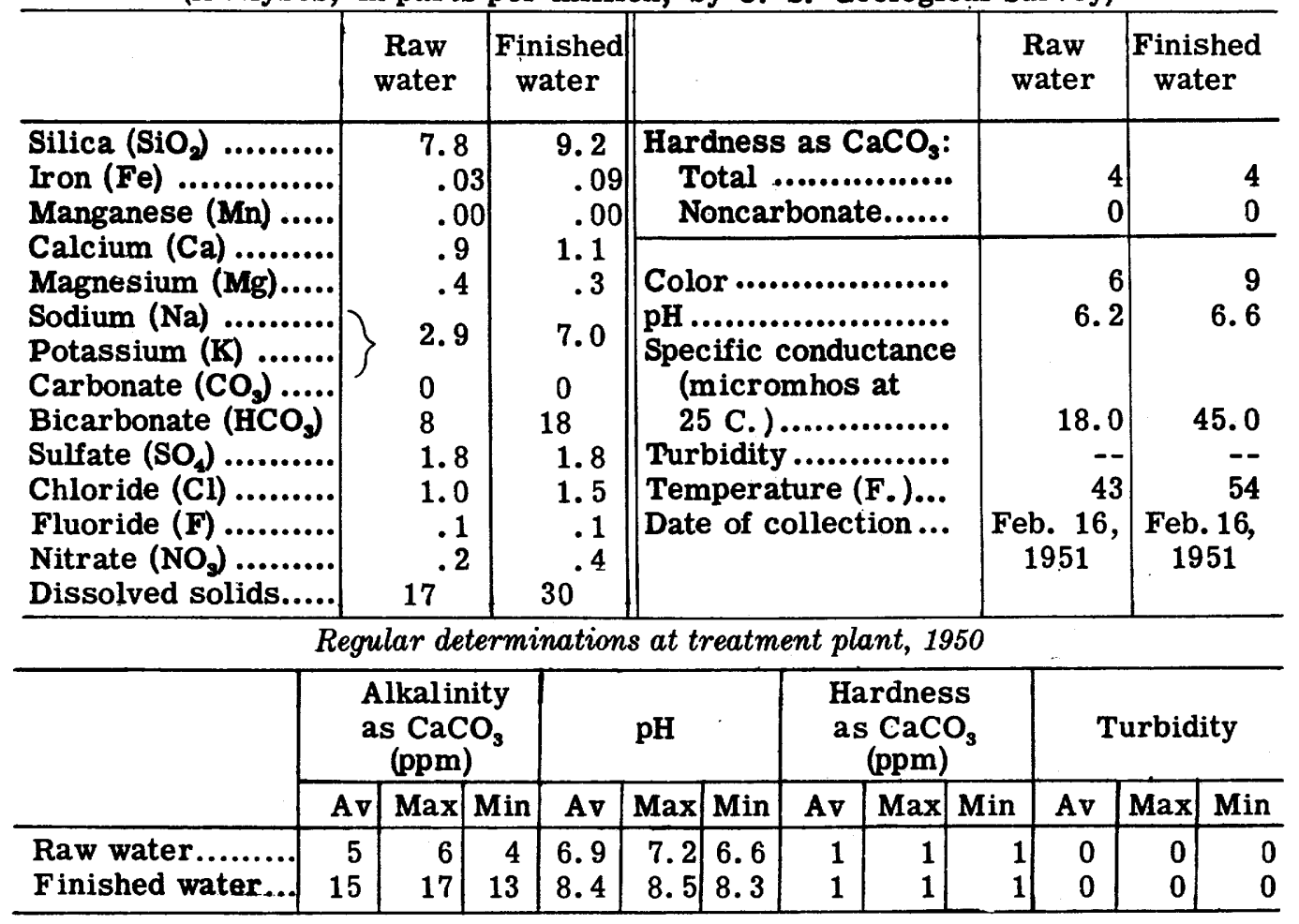


GREENWOOD

(Population, 13, 806)

Ownership: Municipal; also supplies suburban districts. Total population supplied, about 18,000 .

Source: Coronoco Creek impounded in two lakes (1 percent of supply); Saluda River impounded in Lake Greenwood (99 percent of supply). The intake for Coronoco Creek water is 5 miles east of the city, and the intake in Lake Greenwood is about 10 miles east-northeast of the city.

Treatment: Coagulation with alum, sedimentation, rapid sand filtration, addition of lime, and chlorination.

Rated capacity of treatment plant: $3,500,000 \mathrm{gpd}$.

Raw-water storage: Impounding lakes for Coronoco Creek 75, 000, 000 gal; reservoir 10,000,000. Lake Greenwood exceeds 1, 000, 000, 000 gal.

Finished-water storage: Clear wells, 1, 125,000 gal; 1 elevated tank, 300,000 gal. (Additional facilities under construction).

\section{ANALYSES}

(Analyses, in parts per million, by U. S. Geological Survey)

\begin{tabular}{|c|c|c|c|c|c|}
\hline & $\begin{array}{c}\text { Raw } \\
\text { water }^{a}\end{array}$ & $\begin{array}{c}\text { Finished } \\
\text { water }\end{array}$ & & $\begin{array}{c}\text { Raw } \\
\text { water a }\end{array}$ & $\begin{array}{l}\text { Finished } \\
\text { water }\end{array}$ \\
\hline $\begin{array}{l}\text { Silica }\left(\mathrm{SiO}_{2}\right) \\
\text { Iron }(\mathrm{Fe}) \ldots \ldots \ldots \ldots \\
\text { Manganese }(\mathrm{Mn}) \quad \ldots \ldots . . .\end{array}$ & \multirow{2}{*}{$\begin{array}{r}13 \\
.12 \\
.00 \\
2.0 \\
.8\end{array}$} & \multirow{2}{*}{$\begin{array}{r}13 \\
.29 \\
.00 \\
6.0 \\
1.0\end{array}$} & $\begin{array}{r}\text { Hardness as } \mathrm{CaCO}_{3}: \\
\text { Total } \ldots . . . . . . . . . . . . . . \\
\text { Noncarbonate....... }\end{array}$ & $\begin{array}{l}8 \\
0\end{array}$ & $\begin{array}{r}19 \\
0\end{array}$ \\
\hline $\begin{array}{l}\text { Calcium (Ca) ......... } \\
\text { Magnesium (Mg).... }\end{array}$ & & & \multirow{6}{*}{ 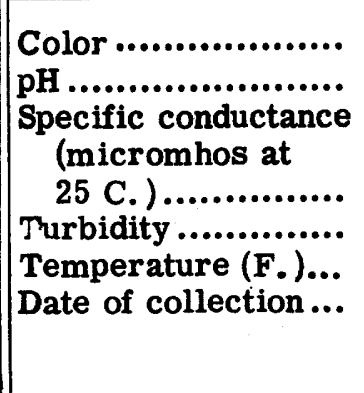 } & 45 & 7 \\
\hline $\begin{array}{l}\text { Sodium (Na) ........... } \\
\text { Potassium (K) ....... }\end{array}$ & 9.1 & 7.9 & & 6.8 & 6.8 \\
\hline $\begin{array}{l}\text { Carbonate }\left(\mathrm{CO}_{3}\right) \ldots . . \\
\text { Bicarbonate }\left(\mathrm{HCO}_{3}\right)\end{array}$ & & & & 72.1 & 76.4 \\
\hline Sulfate $\left(\mathrm{SO}_{4}\right) \ldots \ldots \ldots$ & 3.4 & 7.3 & & -- & -- \\
\hline Chloride (Cl) .......... & 3.9 & 4.2 & & 46 & \\
\hline $\begin{array}{l}\text { Fluoride }(\mathrm{F}) \ldots \ldots \ldots \ldots \\
\text { Nitrate }\left(\mathrm{NO}_{\mathrm{s}}\right) \ldots \ldots \ldots \ldots \\
\text { Dissolved solids...... }\end{array}$ & $47^{.8}$ & $53^{.1}$ & & $\begin{array}{c}\text { Feb. } 16 \\
1951\end{array}$ & $\begin{array}{c}\text { Feb.16 } \\
1951\end{array}$ \\
\hline
\end{tabular}

Regular determinations at treatment plant, 1950

\begin{tabular}{|c|c|c|c|c|c|c|c|c|c|c|c|c|}
\hline & \multicolumn{3}{|c|}{$\begin{array}{c}\text { Alkalinity } \\
\text { as } \mathrm{CaCO} \mathrm{S}_{3} \\
\text { (ppm) }\end{array}$} & \multicolumn{3}{|c|}{$\mathrm{pH}$} & \multicolumn{3}{|c|}{$\begin{array}{c}\text { Hardness } \\
\text { as } \mathrm{CaCO} \\
\text { (ppm) }\end{array}$} & \multicolumn{3}{|c|}{ Turbidity } \\
\hline & Av & Max & Min & Av & Max & $\operatorname{Min}$ & Av & $\operatorname{Max}$ & Min & Av & $\operatorname{Max}$ & Min \\
\hline $\begin{array}{l}\text { Raw water.......... } \\
\text { Finished water... }\end{array}$ & $\begin{array}{l}20 \\
22\end{array}$ & $\begin{array}{l}24 \\
26\end{array}$ & $\begin{array}{l}18 \\
18\end{array}$ & $\begin{array}{l}7.2 \\
7.9\end{array}$ & \begin{tabular}{|l|}
7.3 \\
8.4
\end{tabular} & $\begin{array}{l}6.9 \\
7.7\end{array}$ & $\begin{array}{l}17 \\
20\end{array}$ & \begin{tabular}{|c|}
-- \\
22 \\
\end{tabular} & - & $\begin{array}{l}45 \\
--\end{array}$ & $\begin{array}{l}55 \\
--\end{array}$ & $\begin{array}{l}25 \\
--\end{array}$ \\
\hline
\end{tabular}

${ }^{\text {a Lake Greenwood. }}$ 
GREER

(Population, 5, 050)

Ownership: Municipal; also supplies suburban districts. Total population supplied, about 10,100 .

Source: South Tyger River, 90 percent of supply; enclosed springs, 10 percent of supply. The intake on South Tyger River is about 2.5 miles northeast of the city.

Treatment: Coagulation with alum and lime, sedimentation,. rapid sand filtration, chlorination, Calgon and lime for adjustment of $\mathrm{pH}$. Water from springs: chlorination only.

Rated capacity of treatment plant: $1,500,000 \mathrm{gpd}$.

Raw-water storage: Reservoir, 2, 500,000 gal.

Finished-water storage: Clear well, 625,000 gal; elevated tank, 500,000 gal.

\section{ANALYSES}

(Analyses, in parts per million, by U. S. Geological Survey)

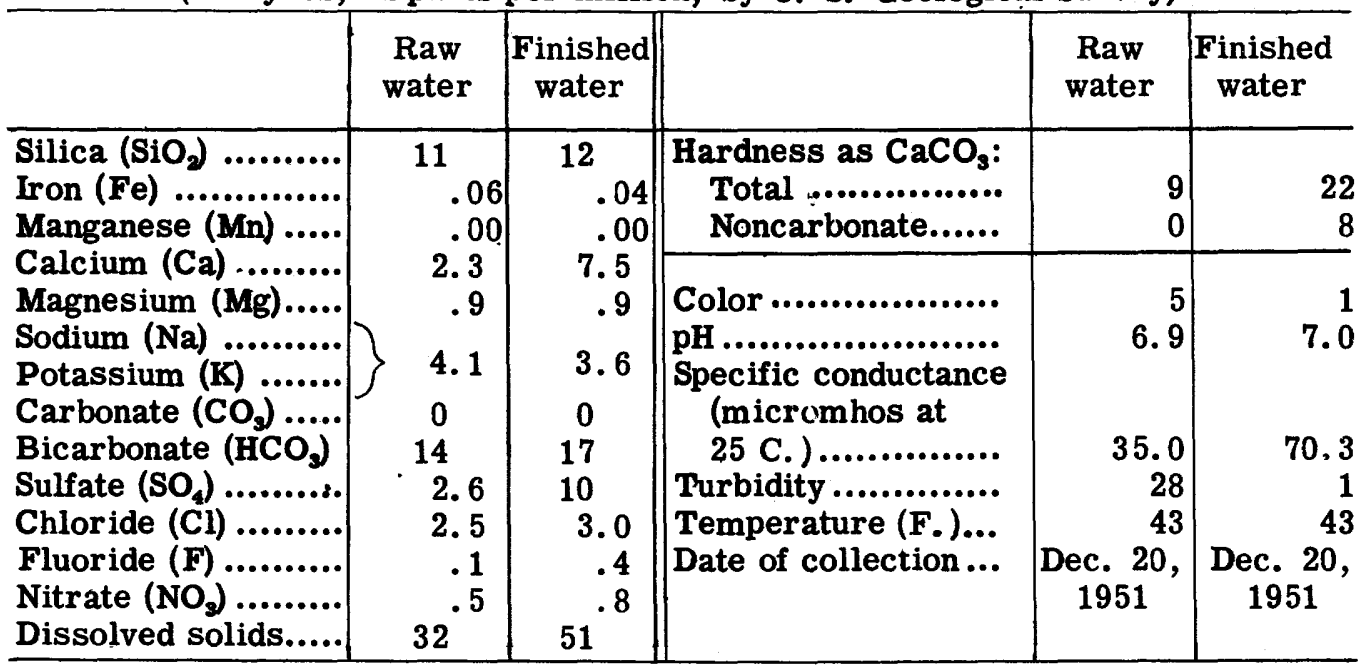




\section{HARTSVILLE}

(Population, 5, 658)

Ownership: Municipal; also supplies suburban districts. Total population supplied, about 11,000.

Source: 12 wells: 2 Layne wells (old well at plant, new well at center of town) 240 and $375 \mathrm{ft}$ deep; 2 wells at plant about $180 \mathrm{ft}$ deep; 4 wells at plant, 160 to $180 \mathrm{ft}$ deep; 4 wells drilled inside of the four last named wells to a depth of $220 \mathrm{ft}$. The yield of the Layne wells is reported to be 814 and $800 \mathrm{gpm}$. The. Layne well at the plant is used all of the time. The other wells of low yield, pump into a reservoir and are used for an auxiliary or emergency supply.

Treatment: Calgon, caustic soda for adjustment of $\mathrm{pH}$.

Rated capacity of treatment plant: 1,500,000 gpd.

Raw-water storage: Reservoir, 500,000 gal.

Finished-water storage: 2 elevated tanks, 300,000 and 75,000 gal.

\section{ANALYSES}

(Analyses, in parts per million, by U. S. Geological Survey)

\begin{tabular}{|c|c|c|c|c|c|}
\hline & $\begin{array}{c}\text { Raw } \\
\text { water a }\end{array}$ & $\begin{array}{r}\text { Finished } \\
\text { water }\end{array}$ & & $\begin{array}{c}\text { Raw } \\
\text { water }\end{array}$ & $\begin{array}{l}\text { Finished } \\
\text { water a }\end{array}$ \\
\hline \multirow{9}{*}{ 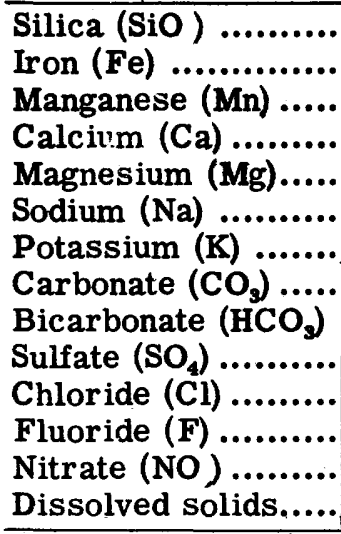 } & \multirow{2}{*}{$\begin{array}{c}8.7 \\
.14 \\
.00 \\
.7 \\
.3\end{array}$} & \multirow{2}{*}{$\begin{array}{l}8.9 \\
.18 \\
.00 \\
.8 \\
.3\end{array}$} & $\begin{array}{r}\text { Hardness as } \mathrm{CaCO}_{3} \\
\text { Total ................. } \\
\text { Noncarbonate...... } \\
\end{array}$ & $\begin{array}{l}3 \\
1\end{array}$ & $\begin{array}{l}3 \\
0 \\
\end{array}$ \\
\hline & & & \multirow{8}{*}{$\begin{array}{l}\text { Color ..................... } \\
\text { pH ...................... } \\
\text { Specific conductance } \\
\text { (micromhos at } \\
25 \text { C. )................ } \\
\text { Turbidity ............. } \\
\text { Temperature (F.)... } \\
\text { Date of collection.... }\end{array}$} & $\begin{array}{r}1 \\
5.1\end{array}$ & \multirow[t]{2}{*}{$\begin{array}{r}2 \\
6.7\end{array}$} \\
\hline & 1.5 & 11 & & & \\
\hline & & $\begin{array}{r}0 \\
25\end{array}$ & & 16.8 & 49.9 \\
\hline & 1.6 & 1.9 & & 0 & 0 \\
\hline & 1.6 & 2.0 & & 65 & 64 \\
\hline & .0 & .3 & & Jan. 10, & Jan. 10, \\
\hline & .0 & .0 & & & \\
\hline & 17 & 37 & & & \\
\hline \multirow{2}{*}{\multicolumn{4}{|c|}{ 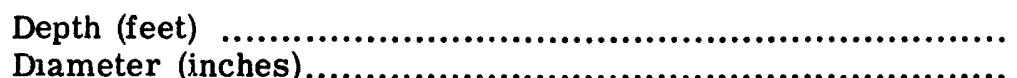 }} & 240 & 240 \\
\hline \multirow{2}{*}{\multicolumn{4}{|c|}{ 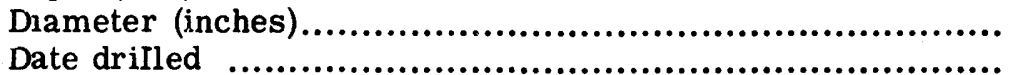 }} & 20 & 20 \\
\hline & & & & 1941 & 1941 \\
\hline \multicolumn{4}{|c|}{ Percent of supply } & -- & -- \\
\hline
\end{tabular}

a Layne well at plant. 


\section{LAURENS}

(Population, 8, 658)

Ownership: Municipal; also supplies suburban districts. Total population supplied, about 11,000 .

Source: Little River 50 percent of supply; Rabon Creek 50 percent of supply.

Treatment: Prechlorination, coagulation with alum and lime, sedimentation, rapid sand filtration, and postchlorination.

Rated capacity of treatment plant: $3,000,000$ gpd.

Raw-water storage: 2 impounding reservoirs, 5, 100, 000 gal.

Finished-water storage: Clear well, 500,000 gal; 3 elevated tanks, 375,000 gal.

\section{ANALYSES}

(Analyses, in parts per million, by U. S. Geological Survey)

\begin{tabular}{|c|c|c|c|}
\hline & $\begin{array}{l}\text { Little River } \\
\text { (raw water) }\end{array}$ & $\begin{array}{r}\text { Rabon Creek } \\
\text { (raw water) }\end{array}$ & $\begin{array}{c}\text { Little River } \\
\text { (finished water) }\end{array}$ \\
\hline 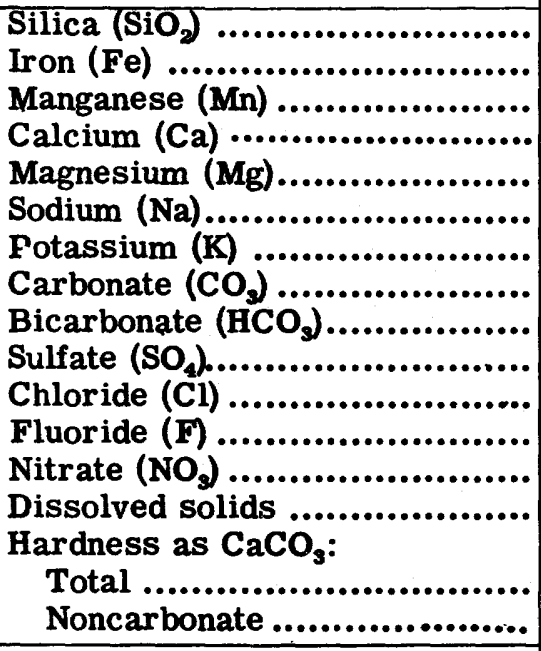 & $\begin{array}{c}0 \\
25 \\
2.7 \\
3.0 \\
.1 \\
.5 \\
52 \\
16 \\
0 \\
\end{array}$ & $\begin{array}{r}0 \\
27 \\
2.3 \\
3.1 \\
.1 \\
52\end{array}$ & $\begin{array}{r}0 \\
30 \\
15 \\
4.9 \\
.1 \\
74^{.6}\end{array}$ \\
\hline 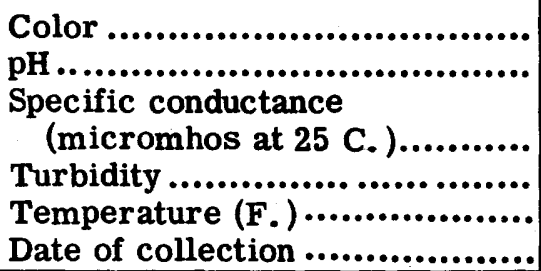 & $\begin{array}{r}7.1 \\
62.7 \\
-- \\
60 \\
\text { Mar. } 9,1951\end{array}$ & Mar. 9, 1951 & $\begin{array}{r}3 \\
6.8 \\
104 \\
-- \\
82 \\
\text { Mar. 9, } 1951 \\
\end{array}$ \\
\hline
\end{tabular}

Regular determinations at treatment plant, 1950

\begin{tabular}{l|r|r|r|r|r|r|r|r|r|r|r|r|r}
\hline & \multicolumn{3}{|c|}{$\begin{array}{c}\text { Alkalinity } \\
\text { as CaCO } \\
\text { (ppm) }\end{array}$} & \multicolumn{4}{c|}{ pH } & \multicolumn{3}{c|}{$\begin{array}{c}\text { Hardness } \\
\text { as CaCO } \\
\text { (ppm) }\end{array}$} & \multicolumn{3}{c}{ Turbidity } \\
\cline { 2 - 14 } & Av & Max & Min & Av & Max & Min & Av & Max & Min & Av & Max & Min \\
\hline Raw water......... & 24 & 30 & 20 & 6.6 & 7.0 & 6.2 & -- & -- & - & 38 & 159 & 14 \\
Finished water... & 24 & 32 & 22 & 7.6 & 7.8 & 7.2 & -- & - & $-7<3$ & -- & - \\
\hline
\end{tabular}


MARION

(Population, 6, 834)

Ownership: Municipal.

Source: 5 wells (1, 3 to 6$) 200,81,150,378$, and $190 \mathrm{ft}$ deep; yield reported to be $100,80,180,300$, and $200 \mathrm{gpm}$.

Treatment: None.

Storage: Reservoir, 240,000 gal; elevated tank, 150,000 gal.

ANALYSES

(Analyses, in parts per million, by U. S. Geologic:! Survey)

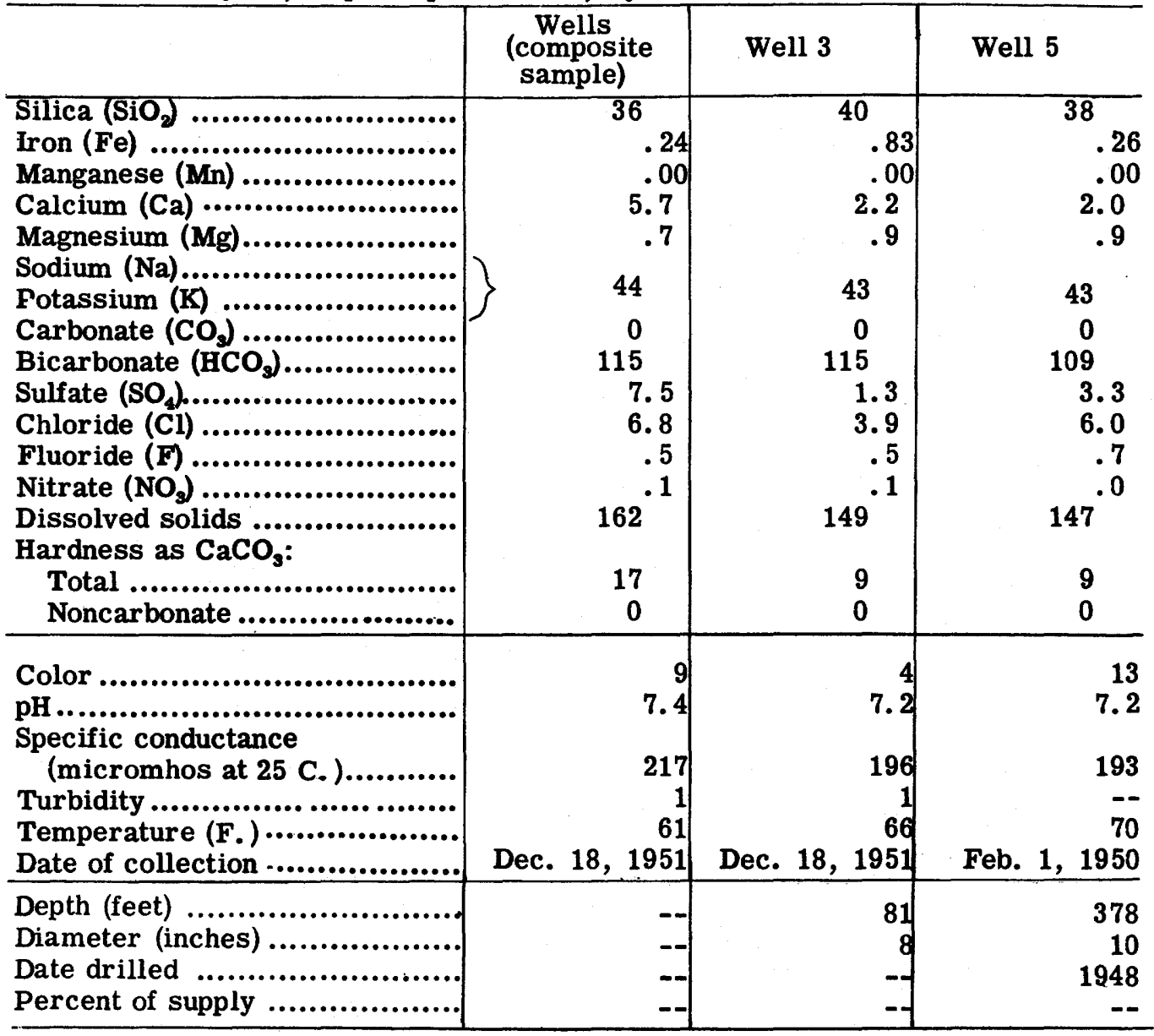


NEWBERRY

(Population, 7, 546)

Ownership: Municipal; also supplies suburban districts. Total population supplied, about 8,500 .

Source: Bush River. The intake on Bush River is about 2.8 miles southwest of the city.

Treatment: Coagulation with alum, chlorination, ammoniation, rapid sand filtration, and adjustment of $\mathrm{pH}$ with lime.

Rated capacity of treatment plant: 2,000,000 gpd.

Raw-water storage: 1,000,000 gal.

Finished-water storage: 3 clear wells, 1,000,000 gal; 2 elevated tanks, 150,000 gal each.

\section{ANALYSES}

(Analyses, in parts per million, by U. S. Geological Survey)

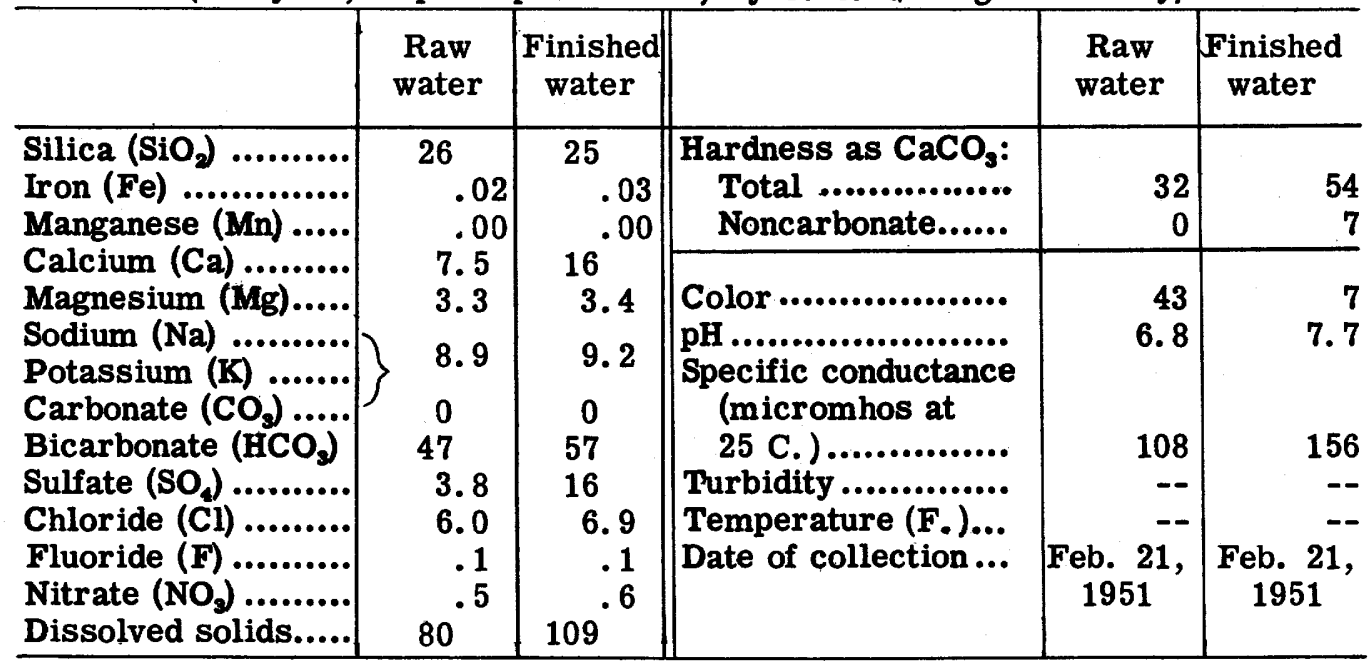

Regular determinations at treatment plant, 1950

\begin{tabular}{|c|c|c|c|c|c|c|c|c|c|c|c|c|}
\hline & \multicolumn{3}{|c|}{$\begin{array}{l}\text { Alkalinity } \\
\text { as CaCO } \\
\text { (ppm) }\end{array}$} & \multicolumn{3}{|c|}{$\mathrm{pH}$} & \multicolumn{3}{|c|}{$\begin{array}{c}\text { Hardness } \\
\text { as } \mathrm{CaCO}_{3} \\
\text { (ppm) }\end{array}$} & \multicolumn{3}{|c|}{ Turbidity } \\
\hline & Av & Max & Min & Av & Max & $\mathrm{Min}$ & Av & $\operatorname{Max}$ & Min & Av & $\operatorname{Max}$ & Min \\
\hline $\begin{array}{l}\text { Raw water......... } \\
\text { Finished water... }\end{array}$ & $\begin{array}{l}45 \\
45\end{array}$ & $\begin{array}{l}50 \\
50\end{array}$ & $\begin{array}{l}40 \\
40\end{array}$ & $\begin{array}{l}7.2 \\
8.2\end{array}$ & \begin{tabular}{|l|}
7.4 \\
8.4
\end{tabular} & $\begin{array}{l}7.0 \\
8.0\end{array}$ & $\begin{array}{l}80 \\
80\end{array}$ & \begin{tabular}{l|}
100 \\
100 \\
\end{tabular} & $\begin{array}{l}60 \\
60 \\
\end{array}$ & $\begin{array}{r}20 \\
5\end{array}$ & $\begin{array}{r}200 \\
8\end{array}$ & $\begin{array}{r}10 \\
3\end{array}$ \\
\hline
\end{tabular}




\section{ORANGEBURG}

(Population, 15, 322)

Ownership: Municipal; also supplies suburban districts. Total population supplied, about 16,000 .

Source: North Fork Edisto River. The intake on North Fork Edisto River is within the city limits.

Treatment: Prechlorination, coagulation with alum and lime, sedimentation, rapid sand filtration, and postchlorination.

Rated capacity of treatment plant: 2, 000,000 gpd.

Raw-water storage: None.

Finished-water storage: 2 clear wells, 750,000 gal; 3 elevated tanks, 150,000, 250,000 , and $50,000 \mathrm{gal}$.

\section{ANALYSES}

(Analyses, in parts per million, by U. S. Geological Survey)

\begin{tabular}{|c|c|c|c|c|c|c|c|c|c|c|c|c|}
\hline & $\begin{array}{c}\text { Raw } \\
\text { water }\end{array}$ & \multicolumn{2}{|c|}{$\begin{array}{c}\text { Finished } \\
\text { water }\end{array}$} & & $\begin{array}{c}\text { Raw } \\
\text { water }\end{array}$ & \multicolumn{2}{|c|}{$\begin{array}{c}\text { Finished } \\
\text { water }\end{array}$} \\
\hline \multirow{7}{*}{\multicolumn{2}{|c|}{ 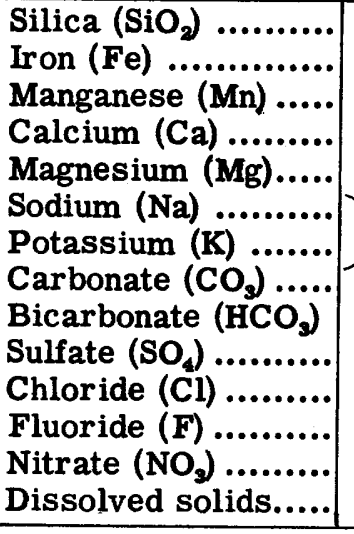 }} & \multirow{2}{*}{$\begin{array}{l}4.7 \\
.12 \\
.00 \\
2.4 \\
.7\end{array}$} & \multirow{2}{*}{\multicolumn{2}{|c|}{$\begin{array}{r}1.4 \\
.03 \\
.00 \\
10\end{array}$}} & \multicolumn{4}{|c|}{$\begin{array}{r}\text { Hardness as } \mathrm{CaCO}_{\mathbf{3}}: \\
\text { Total ................. } \\
\text { Noncarbonate...... } \\
\end{array}$} & & & \multicolumn{2}{|r|}{$\begin{array}{l}27 \\
13\end{array}$} \\
\hline & & & & & \multirow{6}{*}{\multicolumn{4}{|c|}{ 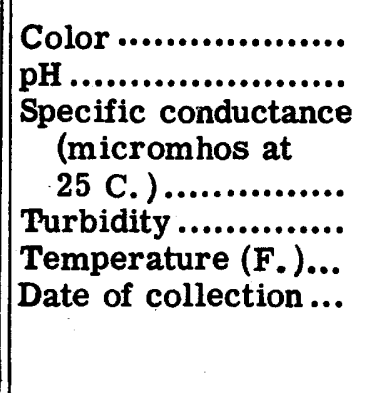 }} & \multirow{2}{*}{\multicolumn{2}{|c|}{$\begin{array}{r}22 \\
5.8\end{array}$}} & \multirow{2}{*}{\multicolumn{2}{|c|}{$\begin{array}{r}2 \\
6.4\end{array}$}} \\
\hline & & \multicolumn{2}{|c|}{3.3} & 3.1 & & & & & & & & \\
\hline & & \multirow{2}{*}{$\begin{array}{r}0 \\
10\end{array}$} & \multicolumn{2}{|c|}{$\begin{array}{r}0 \\
18\end{array}$} & & & & & & 30.2 & \multicolumn{2}{|r|}{77.8} \\
\hline & & & & 8.7 & & & & & & -- & & - \\
\hline & & 3.6 & & 7.2 & & & & & & & & \\
\hline & & $27^{.1}$ & & ${ }_{50}^{.0}$ & & & & & & $\underset{1951}{\operatorname{Mar} .6,}$ & \multicolumn{2}{|c|}{$\underset{1951}{\operatorname{Mar} .6,}$} \\
\hline \multicolumn{13}{|c|}{ Regular determinations at treatment plant, 1950} \\
\hline & \multicolumn{3}{|c|}{$\begin{array}{l}\text { Alkalinity } \\
\text { as CaCO } \\
\text { (ppm) }\end{array}$} & \multicolumn{3}{|c|}{ pH } & \multicolumn{3}{|c|}{$\begin{array}{c}\text { Hardness } \\
\text { as } \mathrm{CaCO} \\
\text { (ppm) }\end{array}$} & \multicolumn{3}{|c|}{$\begin{array}{c}\text { Tempera- } \\
\text { ture } \\
\left({ }^{\circ} \mathrm{F} .\right)\end{array}$} \\
\hline & Av & $\operatorname{Max}$ & Min & Av & $\operatorname{Max}$ & Min & Av & $\operatorname{Max}$ & Min & Av & $\operatorname{Max}$ & Min \\
\hline Raw water......... & 5 & -- & -- & 5.8 & -- & -- & -- & -- & -- & 48 & 59 & 35 \\
\hline & 13 & -- & -- & 8. 7 & -- & -- & 40 & 55 & 28 & -- & -- & -- \\
\hline
\end{tabular}


ROCK HILL

(Population, 24, 502)

Ownership: Municipal; also supplies suburban districts. Total population supplied, about 27,000.

Source: Catawba River. The intake in the river is about 5 miles north of the city. Treatment: Coagulation with alum, activated carbon, sedimentation, rapid sand filtration, ammoniation, and chlorination.

Rated capacity of treatment plant: 4,000,000 gpd.

Raw-water storage: None.

Finished-water storage: Clear well, 1,000,000 gal; elevated tank, 1, 500,000 gal.

\section{ANALYSES}

(Analyses, in parts per million, by U. S. Geological Survey)

\begin{tabular}{|c|c|c|c|c|c|}
\hline & $\begin{array}{l}\text { Raw } \\
\text { water }\end{array}$ & $\begin{array}{c}\text { Finished } \\
\text { water }\end{array}$ & & $\begin{array}{l}\text { Raw } \\
\text { water }\end{array}$ & $\begin{array}{c}\text { Finished } \\
\text { water }\end{array}$ \\
\hline $\begin{array}{l}\text { Silica }\left(\mathrm{SiO}_{2}\right) \\
\text { Iron }(\mathrm{Fe}) \ldots \ldots \ldots \ldots . . . \ldots . . . . . . \\
\text { Manganese }(\mathrm{Mn}) \ldots \ldots\end{array}$ & $\begin{array}{r}12 \\
.02 \\
.00\end{array}$ & $\begin{array}{c}9.6 \\
.02 \\
.05\end{array}$ & $\begin{array}{r}\text { Hardness as } \mathrm{CaCO}_{3}: \\
\text { Total .................. } \\
\text { Noncarbonate...... }\end{array}$ & $\begin{array}{r}14 \\
0 \\
\end{array}$ & $\begin{array}{r}13 \\
0 \\
\end{array}$ \\
\hline $\begin{array}{l}\text { Calcium }(\mathrm{Ca}) . . . . . . . \\
\text { Magnesium (Mg).... }\end{array}$ & $\begin{array}{l}3.4 \\
1.4\end{array}$ & $\begin{array}{l}3.2 \\
1.3\end{array}$ & Color................... & 3 & 3 \\
\hline $\begin{array}{l}\text { Sodium (Na) .......... } \\
\text { Potassium (K) ....... }\end{array}$ & 5.9 & 12 & pH ........................ & 6.7 & 6.7 \\
\hline $\begin{array}{l}\text { Carbor ate }\left(\mathrm{CO}_{3}\right) \ldots . . \\
\text { Bicarbonate }\left(\mathrm{HCO}_{3}\right)\end{array}$ & $\begin{array}{r}0 \\
-20\end{array}$ & $\begin{array}{r}0 \\
28\end{array}$ & $\begin{array}{l}\text { (micromhos at } \\
25 \text { C. }) . . . \ldots \ldots \ldots \ldots\end{array}$ & 58.2 & 83.8 \\
\hline Sulfate $\left(\mathrm{SO}_{4}\right) \ldots . . . . .$. & 4.9 & 9.5 & Turbidity ............... & -- & -- \\
\hline Chloride (Cl) .......... & 3.2 & 3.5 & Temperature (F.)... & 46 & 44 \\
\hline Fluoride (F) ........... & .1 & & Date of collection... & Feb. 14, & Feb. 14, \\
\hline Nitrate $\left(\mathrm{NO}_{3}\right) \ldots . . . .$. & .9 & .5 & & 1951 & 1951 \\
\hline Dissolved solids.... & 42 & 54 & & & \\
\hline
\end{tabular}

Regular determinations at treatment plant, 1950

\begin{tabular}{|c|c|c|c|c|c|c|c|c|c|c|c|c|}
\hline & \multicolumn{3}{|c|}{$\begin{array}{l}\text { Alkalinity } \\
\text { as CaCo } \\
\text { (ppm) }\end{array}$} & \multicolumn{3}{|c|}{$\mathrm{pH}$} & \multicolumn{3}{|c|}{$\begin{array}{l}\text { Hardness } \\
\text { as } \mathrm{CaCO}_{3} \\
(\mathrm{ppm})\end{array}$} & \multicolumn{3}{|c|}{ Turbidity } \\
\hline & Av & $\operatorname{Max}$ & Min & Av & $\operatorname{Max}$ & Min & Av & $\operatorname{Max}$ & Min & Av & $\operatorname{Max}$ & Min \\
\hline $\begin{array}{l}\text { Raw water......... } \\
\text { Finished water... }\end{array}$ & $\begin{array}{l}18 \\
28\end{array}$ & \begin{tabular}{|l|}
22 \\
32 \\
\end{tabular} & $\begin{array}{l}16 \\
26\end{array}$ & $\begin{array}{l}6.8 \\
8.3\end{array}$ & \begin{tabular}{|l|}
7.0 \\
8.5
\end{tabular} & $\begin{array}{l}6.5 \\
8.1\end{array}$ & $\begin{array}{l}14 \\
14\end{array}$ & $\begin{array}{l}16 \\
16\end{array}$ & $\begin{array}{l}12 \\
12\end{array}$ & $\begin{array}{r}25 \\
1\end{array}$ & $\begin{array}{r}500 \\
1\end{array}$ & $\begin{array}{r}20 \\
1\end{array}$ \\
\hline
\end{tabular}




\section{SPART ANBURG \\ (Population, 36, 795)}

Ownership: Municipal; also supplies suburban areas. Total population supplied, about 60,000 .

Source: South Pacolet River impounded. The intake is about 13 miles north of the city.

Treatment: Coagulation with alum and lime, sedimentation, rapid sand filtration, chlorination, adjustment of $\mathrm{pH}$ with soda-ash, and addition of phosphate for corrosion control.

Rated capacity of treatment plant: $10,000,000$ gpd.

Raw-water storage: Impounding reservoir, 879,000, 000 gal.

Finished-water storage: Clear well, 1,000,000 gal; 2 reservoirs, 6, 000,000 gal; elevated tank, 1,500,000 gal. Additional facilities: at Camp Croft 1 standpipe, 2,300,000 gal; at Camp Wadsworth 1 reservoir, 216,000 gal.

\section{ANALYSES}

(Analyses, in parts per million, by U. S. Geological Survey)

\begin{tabular}{|c|c|c|c|c|c|}
\hline & $\begin{array}{c}\text { Raw } \\
\text { water }\end{array}$ & $\begin{array}{c}\text { Finished } \\
\text { water }\end{array}$ & & $\begin{array}{c}\text { Raw } \\
\text { water }\end{array}$ & $\begin{array}{c}\text { Finished } \\
\text { water }\end{array}$ \\
\hline \multirow{8}{*}{ 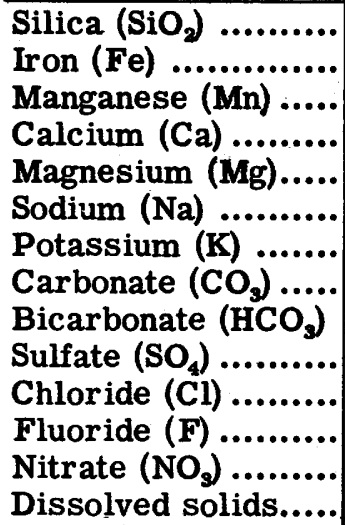 } & \multirow{2}{*}{$\begin{array}{r}12 \\
.04 \\
.00 \\
5.7 \\
.9\end{array}$} & \multirow{2}{*}{$\begin{array}{c}11 \\
: 04 \\
.00 \\
5.6 \\
1.0\end{array}$} & 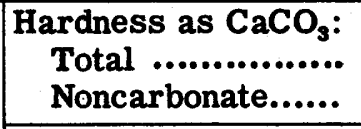 & $\begin{array}{r}18 \\
6\end{array}$ & $\begin{array}{r}18 \\
5\end{array}$ \\
\hline & & & Color...................... & \multirow{2}{*}{$\begin{array}{r}3 \\
6.5\end{array}$} & \multirow{2}{*}{$\begin{array}{r}7 \\
6.3\end{array}$} \\
\hline & .6 & 4.9 & & & \\
\hline & & & & 35.1 & 64.6 \\
\hline & 2.9 & 10 & Turbidity ................ & -- & -- \\
\hline & 2.2 & 3.1 & Temperature (F.)... & 41 & \\
\hline & $\begin{array}{l}.1 \\
.7\end{array}$ & $\begin{array}{l}.1 \\
.6\end{array}$ & Date of collection... & $\begin{array}{c}\text { Feb. } 16 \\
1951\end{array}$ & $\begin{array}{c}\text { Feb. 16, } \\
1951\end{array}$ \\
\hline & 34. & 46 & & & \\
\hline
\end{tabular}

Regular determinations at treatment plant, 1950

\begin{tabular}{|c|c|c|c|c|c|c|c|c|c|c|c|c|}
\hline & \multicolumn{3}{|c|}{$\begin{array}{l}\text { Alkalinity } \\
\text { as CaCO } \\
\text { (ppm) }\end{array}$} & \multicolumn{3}{|c|}{$\mathrm{pH}$} & \multicolumn{3}{|c|}{$\begin{array}{c}\text { Hardness } \\
\text { as } \mathrm{CaCO} \\
\text { (ppm) }\end{array}$} & \multicolumn{3}{|c|}{ Turbidity } \\
\hline & Av & $\operatorname{Max}$ & Min & Av & $\operatorname{Max}$ & Min & Av & $\operatorname{Max}$ & Min & Av & $\operatorname{Max}$ & Min \\
\hline $\begin{array}{l}\text { Raw water.......... } \\
\text { Finished water... }\end{array}$ & \begin{tabular}{l|}
10 \\
16
\end{tabular} & $\begin{array}{l}14 \\
14\end{array}$ & $\begin{array}{r}8 \\
18\end{array}$ & $\begin{array}{l}6.6 \\
7.2\end{array}$ & $\begin{array}{l}7.0 \\
7.3\end{array}$ & $\begin{array}{l}6.1 \\
7.1\end{array}$ & $\begin{array}{l}0.8 \\
1.0\end{array}$ & -- & $\begin{array}{l}-- \\
--\end{array}$ & $\begin{array}{l}100 \\
2.5\end{array}$ & $\begin{array}{l}500 \\
1.0\end{array}$ & $\begin{array}{r}20 \\
5.0\end{array}$ \\
\hline
\end{tabular}




\section{SUMTER}

(Population, 20, 185)

Ownership: Municipal.

Source: 7 wells: 4 deep wells (1 to 4) $625,625,625$, and $615 \mathrm{ft}$ deep; 1 battery of 3 shallow wells. The yield of the deep wells is reported to be $1,400,1,580$, 1,000 and $1,650 \mathrm{gpm}$, and of the battery of 3 shallow wells, $550 \mathrm{gpm}$.

Treatment: Water from wells 2 and 3 treated with calgon; water from shallow wells, not treated.

Raw-water storage: None.

Finished-water storage: Standpipe, 335,000 gal; elevated tank, 100,000 gal; reservoir, 1,000,000 gal.

\section{ANALYSES}

(Analyses, in parts per million, by U. S. Geological Survey)

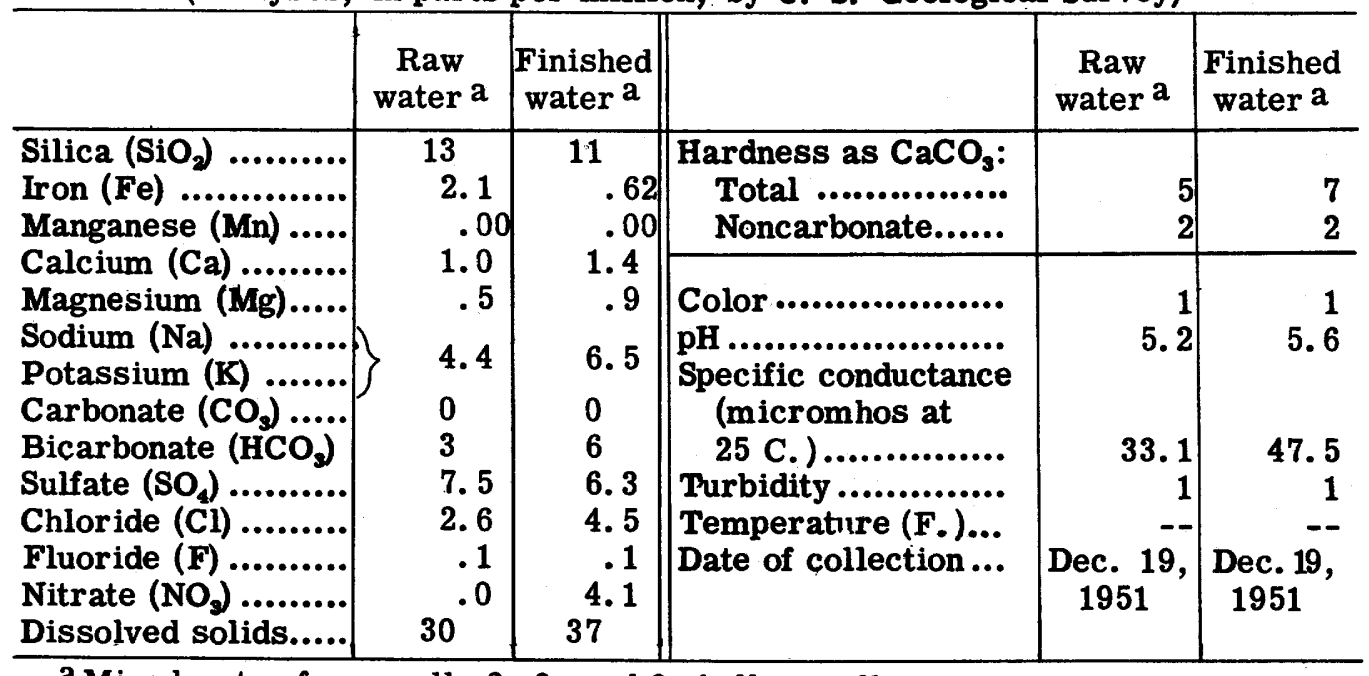

a Mixed water from wells 2, 3, and 3 shallow wells. 


\section{UNION \\ (Population, 9, 730)}

Ownership: Municipal; also supplies suburban areas. Total population supplied, about 11,000 .

Source: Broad River 50 percent of supply; Big Browns Creek and Unnamed Creek 50 percent of supply. The inţake on Broad River is about 9 miles north of the city.

Treatment: Coagulation with alum, soda-ash, chlorination, sedimentation, rapid sand filtration, and ammoniation.

Rated capacity of treatment plant: 4,000,000 gpd.

Raw-water storage: Impounding reservoirs, 65, 000, 000 gal.

Finished-water storage: 2 clear wells, 500,000 and 275,000 gal; standpipe, 250, 000; 1 elevated tank, 400,000 gal.

\section{ANALYSES}

(Analyses, in parts per million, by U. S. Geological Survey)

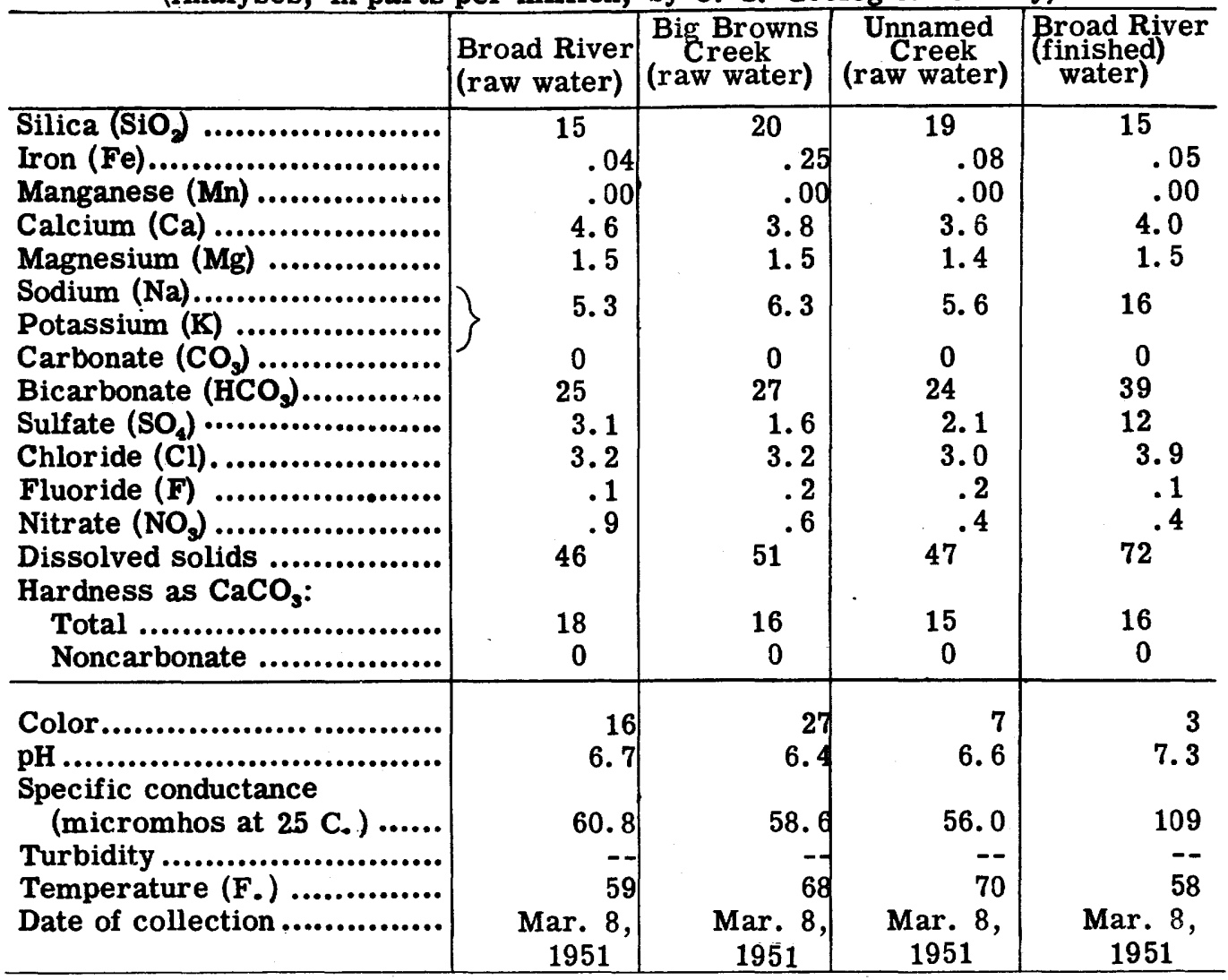

Regular determinations at treatment plant, 1950

\begin{tabular}{|c|c|c|c|c|c|c|c|c|c|c|c|c|}
\hline & \multicolumn{3}{|c|}{$\begin{array}{c}\text { Alkalinity } \\
\text { as CaCO } \\
\text { (ppm) }\end{array}$} & \multicolumn{3}{|c|}{ pH } & \multicolumn{3}{|c|}{$\begin{array}{c}\text { Hardness } \\
\text { as } \mathrm{CaCO}_{3} \\
\text { (ppm) }\end{array}$} & \multicolumn{3}{|c|}{ Turbidity } \\
\hline & Av & $\operatorname{Max}$ & Min & Av & $\operatorname{Max}$ & Min & $\mathbf{A v}$ & $\operatorname{Max}$ & Min & Av & Max & Min \\
\hline $\begin{array}{l}\text { Raw water......... } \\
\text { Finished water.. }\end{array}$ & $\begin{array}{l}22 \\
30\end{array}$ & $\begin{array}{l}20 \\
32\end{array}$ & $\begin{array}{l}22 \\
30\end{array}$ & $\begin{array}{l}7.2 \\
7\end{array}$ & $\begin{array}{l}7.4 \\
7.4\end{array}$ & $\begin{array}{l}6.8 \\
7.2\end{array}$ & $\overline{14}$ & \begin{tabular}{|c|}
-- \\
14
\end{tabular} & $\overline{14}$ & $\begin{array}{l}80 \\
--\end{array}$ & $\begin{array}{r}300 \\
--\end{array}$ & $\begin{array}{l}80 \\
--\end{array}$ \\
\hline
\end{tabular}




\section{ALEXANDRIA \\ (Population, 61, 787)}

Ownership: Alexandria Water Co. and Virginia Water Co., which distributes water in Fairfax County (both controlled by the American Water Works Co. Inc. , Philadelphia, Pa.). Supplies also suburban districts and communities in Fairfax County. Total population supplied, about 76,000.

Source: Occoquan Creek impounded. Auxiliary supply, 3 wells of total capacity of $1,000,000 \mathrm{gpd}$. The well supply is used during summer months.

Treatment: Prechlorination, coagulation with alum and lime, activated carbon as required, sedimentation, rapid sand filtration, postchlorination as required, and adjustment of $\mathrm{pH}$.

Rated capacity of treatment plant: $8,800,000 \mathrm{gpd}$.

Raw-water storage: 1,750,000,000 gal.

Finished-water storage: Clear wells, 400,000 gal; undergound reservoir, 700,000 gal; open reservoir, 16,000,000 gal; other facilities, 3, 300,000 gal.

The intake dam on Occoquan Creek and the treatment plant are about 20 miles south of the city. This dam which is about $1,800 \mathrm{ft}$ upstream from tidewater impounds about 55,000,000 gal. Another impounding dam about 7 miles above tidewater impounds an estimated 1.5 to 2.0 billion gal. The raw water is pumped through a 30 in. main to the treatment plant about $\frac{1}{2}$ mile distant on a hill at an elevation of about $245 \mathrm{ft}$. Finished water is pumped through high service pumps to Alexandria, the intervening area, distribution, and storage. The water of Occoquan Creek is subject to rather sudden changes in quality. At times of heavy rains, turbidity changes are rapid and large. Color removal is always a problem.

\section{ANALYSIS}

(Analysis, in parts per million, by U. S. Geological Survey)

\begin{tabular}{|c|c|c|c|}
\hline & $\begin{array}{l}\text { Finished } \\
\text { water } \\
\text { (city tap) }\end{array}$ & & $\begin{array}{l}\text { Finished } \\
\text { water } \\
\text { (city tap) }\end{array}$ \\
\hline \multirow{2}{*}{ 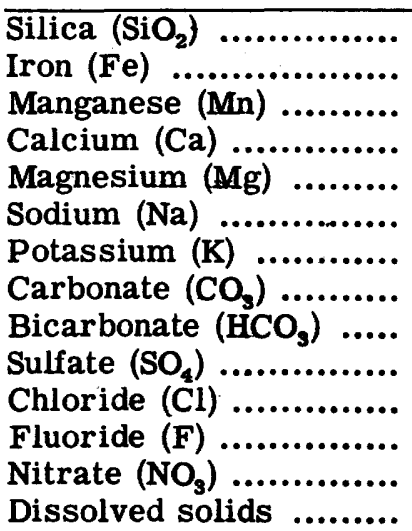 } & \multirow{2}{*}{$\begin{array}{c}9.5 \\
.09 \\
.19 \\
14 \\
2.4 \\
4.0 \\
.9 \\
0 \\
34 \\
19 \\
4.2 \\
.0 \\
.6 \\
74\end{array}$} & $\begin{array}{l}\text { Hardness as } \mathrm{CaCO}_{\mathbf{3}}: \\
\quad \text { Total ........................... } \\
\text { Noncarbonate ............ }\end{array}$ & $\begin{array}{l}43 \\
17\end{array}$ \\
\hline & & 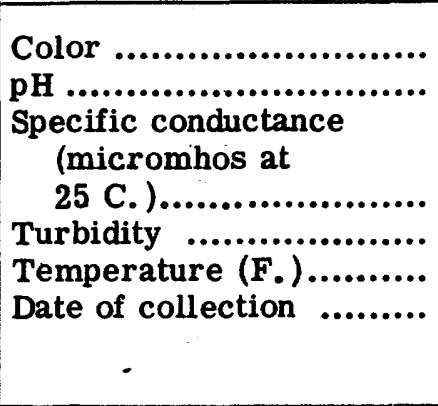 & $\begin{array}{r}121 \\
.6 \\
\text { May } 18, \\
1951\end{array}$ \\
\hline
\end{tabular}

Regular determinations at treatment plant, Jan. 1951

\begin{tabular}{l|r|r|r|r|r|r|r|r|r|r|r|r}
\hline & \multicolumn{3}{|c|}{$\begin{array}{c}\text { Alkalinity } \\
\text { as CaCO, } \\
\text { (ppm) }\end{array}$} & \multicolumn{3}{|c|}{ pH } & \multicolumn{3}{c|}{$\begin{array}{c}\text { Hardness } \\
\text { as CaCO, } \\
\text { (ppm) }\end{array}$} & \multicolumn{3}{c}{ Turbidity } \\
\cline { 2 - 13 } & Av & Max & Min & Av & Max & Min & Av & Max & Min & Av & Max & Min \\
\hline Raw water......... & 24 & 26 & 22 & 7.1 & 7.3 & 7.0 & -- & - & - & 29 & 50 & 16 \\
Finished water... & 29 & 32 & 22 & 8.4 & 8.7 & 7.9 & -- & -- & - & -- & -- & -- \\
\hline
\end{tabular}




\section{ARLINGTON COUNTY}

(Population, 135, 449)

Ownership: The distribution system is owned and operated by Arlington County. Finished water is purchased from Washington, D. C. Supplies also the city of Falls Church, and areas in Fairfax County through Falls Church. Total population supplied, about 158,000 .

Source: Potomac River. Finished water from the Dalecarlia treatment plant. (See Washington, D. C.)

Finished-water storage: 3 ground reservoirs, 4 elevated tanks; total, 10, 500,000 gal.

\section{BRISTOL \\ (Population, 15, 954)}

Ownership: Municipal; also supplies suburban population of about 500. Total population supplied, about 16,500 .

Source: Clear Creek (50 percent of supply); Preston Spring (35 percent of supply); and Mumpower Springs (15 percent of supply).

Treatment: Partial softening with lime, coagulation with copperas (and alum at times of high turbidity), sedimentation, rapid sand filtration, and chlorination. Spring supplies: chlorination.

Rated capacity of treatment plant: 2,000,000 gpd.

Raw-water storage: 5,000,000 gal.

Finished-water storage: 1,000,000 gal.

The city of Bristol is served by two supply systems. Clear Creek and Preston Spring supply one system (low service) and Mumpower Springs the other (high service). Preston Spring is about 6.5 miles northwest of the city. Water from Preston Spring is diverted to the treatment plant for the creek supply which is located near the spring. Chlorinated water from the spring and finished water from the treatment plant flow by gravity in a single pipe line to the city to low service lines. Mumpower Springs (several springs) are about 4 miles north of the city. Water from the springs is collected in an open reservoir about $\frac{1}{4}$ mile below the springs, from which it flows by gravity to the city to high service lines. Chlorine is applied in the gravity line a short distance below the reservoir.

There is an 8 in. pipe line connecting the water supply system of Bristol, Tennessee with the supply system of Bristol, Virginia which can be used in emergencies. 
BRISTOL--Continued

\section{ANALYSES}

(Analyses, in parts per million, by U. S. Geological Survey)

\begin{tabular}{|c|c|c|c|c|}
\hline & $\begin{array}{c}\text { Clear } \\
\text { Creek } \\
\text { (raw water) }\end{array}$ & $\begin{array}{c}\text { Preston } \\
\text { Spring } \\
\text { (raw water) }\end{array}$ & $\begin{array}{l}\text { Finished } \\
\text { water a }\end{array}$ & $\begin{array}{c}\text { Finished } \\
\text { water } b\end{array}$ \\
\hline 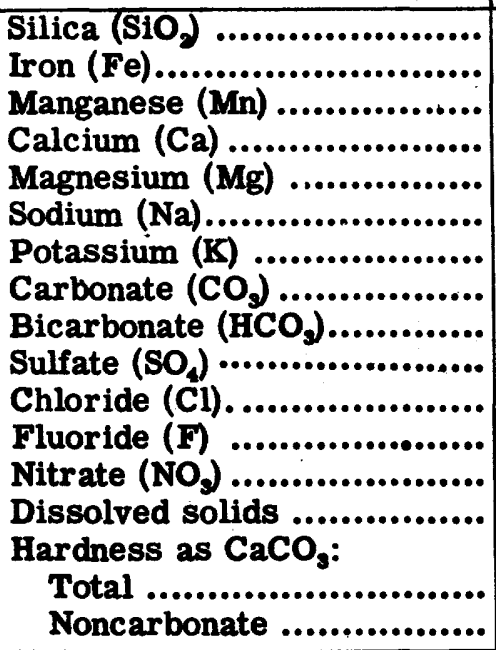 & $\begin{array}{c}3.6 \\
.06 \\
\\
50 \\
-- \\
21 \\
.7 \\
1.2 \\
0 \\
233 \\
21 \\
1.0 \\
.2 \\
2.9 \\
235\end{array}$ & $\begin{array}{c}1.8 \\
.02 \\
57 \\
22 \\
22 \\
.8 \\
1.5 \\
0 \\
245 \\
30 \\
1.4 \\
.2 \\
6.6 \\
271\end{array}$ & $\begin{array}{c}4.0 \\
.02 \\
-- \\
33 \\
21 \\
.7 \\
1.4 \\
0 \\
166 \\
26 \\
2.4 \\
.2 \\
4.5 \\
198\end{array}$ & $\begin{array}{l}2.0 \\
.02 \\
40 \\
19 \\
.- \\
.6 \\
1.3 \\
0 \\
208 \\
6.5 \\
2.8 \\
.2 \\
.8 \\
194 \\
178 \\
7\end{array}$ \\
\hline 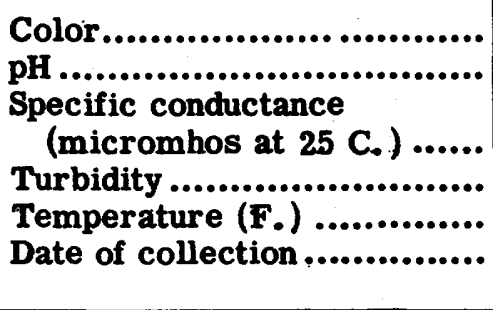 & $\begin{array}{r}0 \\
8.3 \\
384 \\
10 \\
65 \\
\text { Sept. } 12, \\
1951\end{array}$ & $\begin{array}{r}430 \\
0 \\
63 \\
\text { Sept. } 12, \\
1951\end{array}$ & $\begin{array}{r}0 \\
8.0 \\
310 \\
.5 \\
65 \\
\text { Sept. } 12 \text {; } \\
1951\end{array}$ & $\begin{array}{r}321 \\
0 \\
61 \\
\text { Sept. 12, } \\
1951\end{array}$ \\
\hline
\end{tabular}

Regular determinations at treatment plant, 1950

\begin{tabular}{|c|c|c|c|c|c|c|c|c|c|c|c|c|}
\hline & \multicolumn{3}{|c|}{$\begin{array}{l}\text { Alkalinity } \\
\text { as CaCO } \\
\text { (ppm) }\end{array}$} & \multicolumn{3}{|c|}{$\mathbf{p H}$} & \multicolumn{3}{|c|}{$\begin{array}{l}\text { Hardness } \\
\text { as CaCO } \\
\text { (ppm) }\end{array}$} & \multicolumn{3}{|c|}{$\begin{array}{c}\text { Tempera- } \\
\text { ture } \\
\left({ }^{\circ} \mathrm{F} .\right)\end{array}$} \\
\hline & $\mathbf{A v}$ & $\operatorname{Max}$ & Min & Av & $\operatorname{Max}$ & Min & Av & $\operatorname{Max}$ & Min & Av & Max & Min \\
\hline $\begin{array}{l}\text { Raw water......... } \\
\text { Finished water.. }\end{array}$ & $\begin{array}{r}150 \\
41\end{array}$ & $\begin{array}{r}196 \\
58\end{array}$ & $\begin{array}{l}44 \\
15\end{array}$ & $\begin{array}{l}8.2 \\
9.2\end{array}$ & $\begin{array}{l}8.4 \\
9.6\end{array}$ & $\begin{array}{l}8.0 \\
8.9\end{array}$ & $\begin{array}{l}230 \\
120\end{array}$ & $\begin{array}{l}244 \\
170\end{array}$ & $\begin{array}{r}218 \\
82\end{array}$ & 55 & $\begin{array}{l}68 \\
--\end{array}$ & $\begin{array}{l}32 \\
--\end{array}$ \\
\hline
\end{tabular}

a Clear Creek and Preston Spring.

b Mumpower Springs. 


\section{CHARLOTTESVILLE}

(Population, 25, 969)

Ownership: Municipal; also supplies about 2,600 people outside the city limits. Total population supplied, about 28,600 .

Source: Moormans River 90 percent of supply; Ragged Mt. reservoir 10 percent of supply.

Treatment: Prechlorination, coagulation with alum and lime, sedimentation, slow sand filtration, postchlorination, addition of sodium phosphate, and fluoridation with sodium Isilicofluoride. Copper sulphate for the control of algae.

Rated capacity of treatment plant: 3,500,000 gipd.

Raw-water storage: 1,000,000,000 gal.

Finished-water storage: 3,000,000 gal.

The filter plant is located in Charlottesville, on Reservoir Mt. , western edge of town. Water is piped from Moormans River Dam to the filter plant. The pipe line passes Ragged Mt. Reservoir and excess water from Moormans River is diverted, and stored in the Ragged Mt. Reservoir which also receives water from Ragged Mt. watershed. Water from Ragged Mt. Reservoir is used to balance the water needs.

ANALYSES

(Analyses, in parts per million, by U. S. Geological Survey)

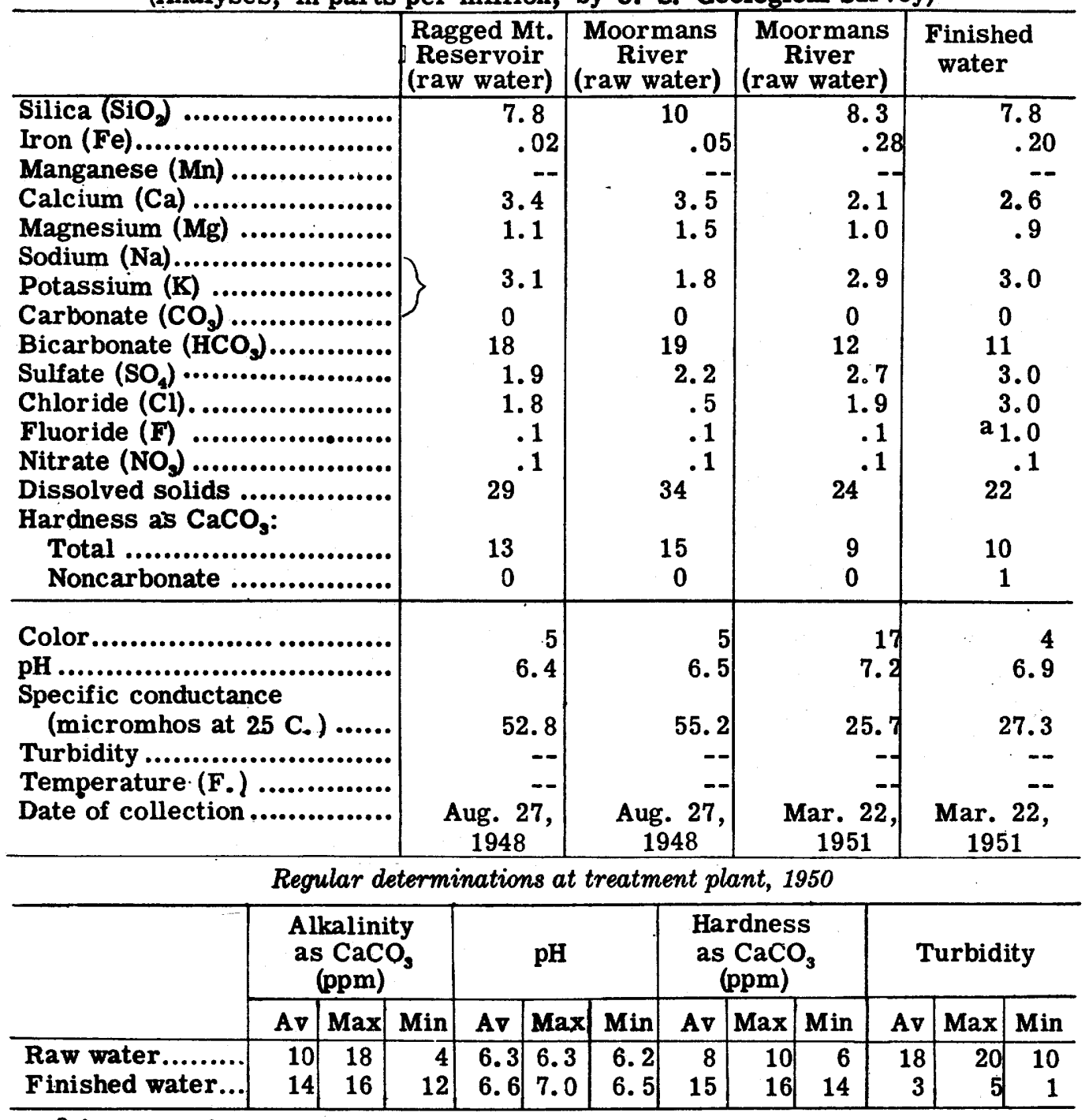

a Average of several determinations, 1952. 
DANVILLE

(Population, 35, 066)

Ownership: Municipal; also supplies suburban population of about 800 . Total population supplied, about 35,900 .

Source: Dan River.

Treatment: Coagulation with alum and lime, activated carbon, aeration (spray), chlorination, sedimentation, rapid sand filtration, postchlorination, ammoniation, and adjustment of $\mathrm{pH}$ with soda ash.

Rated capacity of treatment plant: $6,000,000 \mathrm{gpd}$.

Raw-water storage: 3,000,000,000 gal.

Finished-water storage: 9,000,000 gal.

The treatment plant is on the north edge of the city near the cotton mills. The intake is in the reservoir about 100 yards above the impounding dam of the Riverside and Dan River Cotton Mills.

\section{ANALYSES}

(Analyses, in parts per million, by U. S. Geological Survey)

\begin{tabular}{|c|c|c|c|}
\hline & & $\begin{array}{l}\text { Raw } \\
\text { water }\end{array}$ & $\begin{array}{l}\text { Finished } \\
\text { water }\end{array}$ \\
\hline 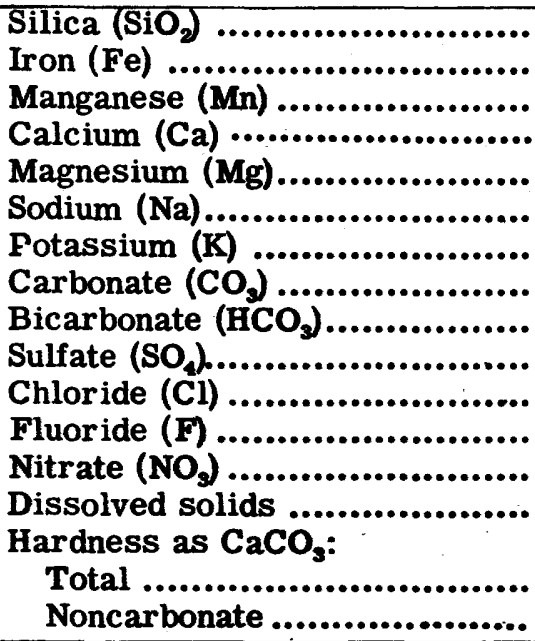 & $\begin{array}{c}13 \\
.01 \\
4.0 \\
1.0 \\
4.5 \\
0 \\
23 \\
4.0 \\
2.0 \\
.1 \\
.4 \\
47 \\
17 \\
0\end{array}$ & $\begin{array}{r}12 \\
--- \\
--- \\
2.7 \\
1.3 \\
0 \\
31 \\
3 \\
2.1 \\
.1 \\
.6 \\
--\end{array}$ & $\begin{array}{c}13 \\
.02 \\
11 \\
11 \\
2.0 \\
4.2 \\
1.4 \\
0 \\
37 \\
8.6 \\
5.2 \\
.1 \\
.2 \\
69\end{array}$ \\
\hline 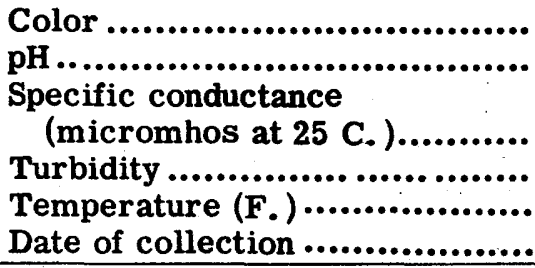 & July 21,1947 & $\begin{array}{r}25 \\
7.3 \\
60.3 \\
4.0 \\
75 \\
\text { Sept. } 11,1951 \\
\end{array}$ & $\begin{array}{r}0 \\
7.7 \\
97.0 \\
.5 \\
. \quad 73 \\
\text { Sept. } 11,1951 \\
\end{array}$ \\
\hline
\end{tabular}

Regular determinations at treatment plant, 1950

\begin{tabular}{l|r|r|r|r|r|r|r|r|r|r|r|r}
\hline & \multicolumn{3}{|c|}{$\begin{array}{c}\text { Alkalinity } \\
\text { as CaCO } \\
\text { (ppm) }\end{array}$} & \multicolumn{3}{c|}{ pH } & \multicolumn{3}{c|}{$\begin{array}{c}\text { Hardiness } \\
\text { as CaCO } \\
\text { (ppm) }\end{array}$} & \multicolumn{3}{c}{ Turbidity } \\
\cline { 2 - 13 } & Av & Max & Min & Av & Max & Min & Av & Max & Min & Av & Max & Min \\
\hline Raw water......... & 22 & 30 & 12 & 7.0 & 7.3 & 6.6 & -- & -- & -- & 1.2 & 4.0 & .2 \\
Finished water... & 30 & 40 & 21 & 8.7 & 9.4 & 8.3 & -- & -- & -- & .3 & 1.8 & .1 \\
\hline
\end{tabular}


FALLS CHURCH

(Population, 7, 535)

Ownership: Distribution system, municipal. The city purchases its water from Arlington County. Supplies about 15,000 people in Fairfax County. Total population supplied, about 22,500 .

Source: Potomac River. Finished water from Dalecarlia treatment plant, Washington, D. C. through Arlington County. Emergency supply: 2 wells $(4,6)$ 430 and $748 \mathrm{ft}$ deep; yield reported to be 40 and $35 \mathrm{gpm}$ respectively; two standby 6 in. connections to Arlington County distribution system in case of interruption of service in main line. (See Washington, D. C.)

Finished-water storage: 2 elevated tanks; 2 stand pipes. Total, 2,000,000 gal.

\section{FREDRICKSBURG}

(Population, 12, 158)

Ownership: Municipal; also supplies a suburban population of about 2,000. Total population supplied, about $14,200$.

Source: Rappahannock River.

Treatment: Primary sedimentation with alum, breakpoint chlorination, coagulation with alum, sedimentation, rapid (anthrafilt) filtration, and adjustment of pH with soda ash.

Rated capacity of treatment plant: $6,000,000$ gpd.

Raw-water storage: 24,000,000 gal.

Finished-water storage: 3,500,000 gal.

Raw water is obtained from a canal that runs by the treatment plant. This canal, and the dam that partially impounds the Rappahannock River, is owned by the Va. Electric \& Power Co. Water from the canal is run into the storage basin and from there is pumped to the treatment plant.

\section{ANALYSES}

(Analyses, in parts per million, by U. S. Geological Survey)

\begin{tabular}{|c|c|c|c|c|c|}
\hline & $\begin{array}{c}\text { Raw } \\
\text { water }\end{array}$ & $\begin{array}{c}\text { Finished } \\
\text { water }\end{array}$ & & $\begin{array}{c}\text { Rad } \\
\text { water }\end{array}$ & $\begin{array}{l}\text { Finished } \\
\text { water }\end{array}$ \\
\hline $\begin{array}{l}\text { Silica }\left(\mathrm{SiO}_{2}\right) \ldots \ldots \ldots \ldots \\
\text { Iron }(\mathrm{Fe}) \ldots \ldots \ldots \ldots . . \\
\text { Manganese }(\mathrm{Mn}) \ldots \ldots\end{array}$ & $\begin{array}{l}7.8 \\
.12 \\
--\end{array}$ & $\begin{array}{c}8.2 \\
.05 \\
--\end{array}$ & $\begin{array}{r}\text { Hardness as } \mathrm{CaCO}_{3} \text { : } \\
\text { Total ................... } \\
\text { Noncarbonate...... }\end{array}$ & $\begin{array}{r}20 \\
0\end{array}$ & $\begin{array}{r}20 \\
0\end{array}$ \\
\hline 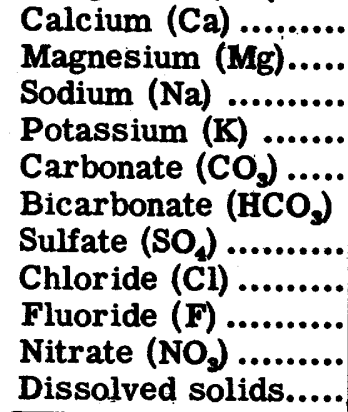 & $\begin{array}{c}5.0 \\
1.8 \\
3.4 \\
1.1 \\
0 \\
27 \\
2.4 \\
2.5 \\
.1 \\
.3\end{array}$ & $\begin{array}{c}5.1 \\
1.7 \\
17 \\
1.1 \\
0 \\
53 \\
7.7 \\
4.9 \\
.1 \\
.1\end{array}$ & $\begin{array}{c}\text { Color .................... } \\
\text { pH ......................... } \\
\text { Specific conductance } \\
\text { (micromhos at } \\
25 \text { C. ) ................ } \\
\text { Turbidity .............. } \\
\text { Temperature (F.)... } \\
\text { Date of collection ... }\end{array}$ & $\begin{array}{r}56.9 \\
25 \\
76 \\
76 \\
\text { Sept. } 13, \\
1951\end{array}$ & $\begin{array}{r}116 \\
1.0 \\
75 \\
\text { Sept. } 13, \\
1951\end{array}$ \\
\hline
\end{tabular}

Regular determinations at treatment plant, 1950

\begin{tabular}{|c|c|c|c|c|c|c|c|c|c|c|c|c|}
\hline & \multicolumn{3}{|c|}{$\begin{array}{l}\text { Alkalinity } \\
\text { as CaCO } \\
\text { (ppm) }\end{array}$} & \multicolumn{3}{|c|}{$\mathrm{pH}$} & \multicolumn{3}{|c|}{$\begin{array}{l}\text { Hardness } \\
\text { as } \mathrm{CaCO}_{3} \\
(\mathrm{ppm})\end{array}$} & \multicolumn{3}{|c|}{ Turbidity } \\
\hline & $\mathbf{A v}$ & Max & Min & Av & Max & $\operatorname{Min}$ & Av & Max & $\mathrm{Min}$ & Av & $\operatorname{Max}$ & Min \\
\hline $\begin{array}{l}\text { Raw water......... } \\
\text { Finished water... }\end{array}$ & $\begin{array}{l}25 \\
36\end{array}$ & \begin{tabular}{|l|}
28 \\
50
\end{tabular} & $\begin{array}{l}12 \\
29\end{array}$ & $\begin{array}{l}7.0 \\
6.7\end{array}$ & \begin{tabular}{|l|}
7.3 \\
8.9 \\
\end{tabular} & $\begin{array}{l}6.4 \\
6.4\end{array}$ & $=$ & -- & $=$ & $\begin{array}{l}64 \\
.7\end{array}$ & $\begin{array}{l}300 \\
1.5\end{array}$ & $\begin{array}{l}15 \\
.5\end{array}$ \\
\hline
\end{tabular}


HARRISONBURG

(Population, 10, 810)

Ownership: Municipal; also supplies about 1,000 people outside the city limits.

Total population supplied, about 11,800 .

Source: Riven Rock Spring (natural flow of Dry River and underflow of Dry River).

Treatment: Chlorination.

Rated capacity of treatment plant: 2,000,000 gpd.

Raw-water storage: None.

Finished-water storage: 21,000,000 gal.

The basin of Dry River is in the George Washington National Forest. The intake works are about 13 miles west of the city. The underflow in the valley of the river is trapped in a gallery by extending the intake dam underground across the valley floor for a distance of $900 \mathrm{ft}$ to a rock cliff on one side of the valley. The underground dam rests on bedrock. Water from the intake works is conducted through a 14 in. pipe line to the chlorinating plant about $\frac{1}{4}$ mile below the intake dam. The chlorinated water is piped to the distribution system and concrete storage reservoirs in the city through two interconnected parallel pipe lines. The supply is by gravity throughout.

\section{ANALYSES}

(Analyses, in parts per million, by U. S. Geological Survey)

\begin{tabular}{|c|c|c|c|c|c|}
\hline & $\begin{array}{c}\text { Raw } \\
\text { water }\end{array}$ & $\begin{array}{c}\text { Finished } \\
\text { water }\end{array}$ & & $\begin{array}{c}\text { Raw } \\
\text { water }\end{array}$ & $\begin{array}{c}\text { Finished } \\
\text { water }\end{array}$ \\
\hline $\begin{array}{l}\text { Silica }\left(\mathrm{SiO}_{2}\right) \\
\text { Iron }(\mathrm{Fe}) \ldots \ldots \ldots \ldots \\
\text { Manganese }(\mathrm{Mn}) \ldots \ldots . . . . .\end{array}$ & $\begin{array}{l}5.3 \\
.07 \\
--\end{array}$ & $\begin{array}{c}6.0 \\
.05 \\
--\end{array}$ & $\begin{array}{r}\text { Hardness as } \mathrm{CaCO}_{3} \text { : } \\
\text { Total ................... } \\
\text { Noncarbonate...... }\end{array}$ & $\begin{array}{l}7 \\
1\end{array}$ & $\begin{array}{l}8 \\
2\end{array}$ \\
\hline 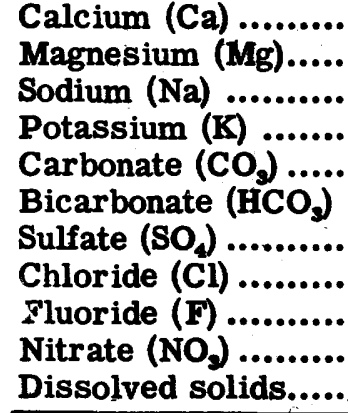 & $\begin{array}{l}1.4 \\
.9 \\
1.0 \\
.6 \\
0 \\
8 \\
3.6 \\
1.0 \\
.0 \\
.1\end{array}$ & $\begin{array}{l}1.4 \\
1.0 \\
1.0 \\
.7 \\
0 \\
7 \\
3.7 \\
1.8 \\
.0 \\
.1\end{array}$ & $\begin{array}{l}\text { Color } . . . . . . . . . . . . . . . . \\
\text { pH ......................... } \\
\text { Specific conductance } \\
\text { (micromhos at } \\
25 \text { C.) ................. } \\
\text { Turbidity ............. } \\
\text { Temperature (F.)... } \\
\text { Date of collection ... }\end{array}$ & $\begin{array}{r}23.6 \\
0 \\
61 \\
\text { Sept.12, } \\
1951\end{array}$ & $\begin{array}{r}5 \\
6.4 \\
\\
25.0 \\
0 \\
61 \\
\text { Sept. } 12, \\
1951\end{array}$ \\
\hline
\end{tabular}




\section{HOP EWELL}

(Population, 10, 219)

Ownership: Old Dominion Water Corp; also supplies about 5,000 people outside the city limits, and the population in Camp Lee, Prison Camp, and Bland sanitary Dist. Total known population supplied, about 15, 200.

Source: Appomattox River.

Treatment: Prechlorination, coagulation with alum, sedimentation, rapid sand filtration, postchlorination, and adjustment of $\mathrm{pH}$ with lime.

Rated capacity of treatment plant: $30,000,000$ gpd.

Raw-water storage: --

Finished-water storage: 3,000,000,000 gal.

\section{ANALYSES}

(Analyses, in parts per million, by U. S. Geological Survey)

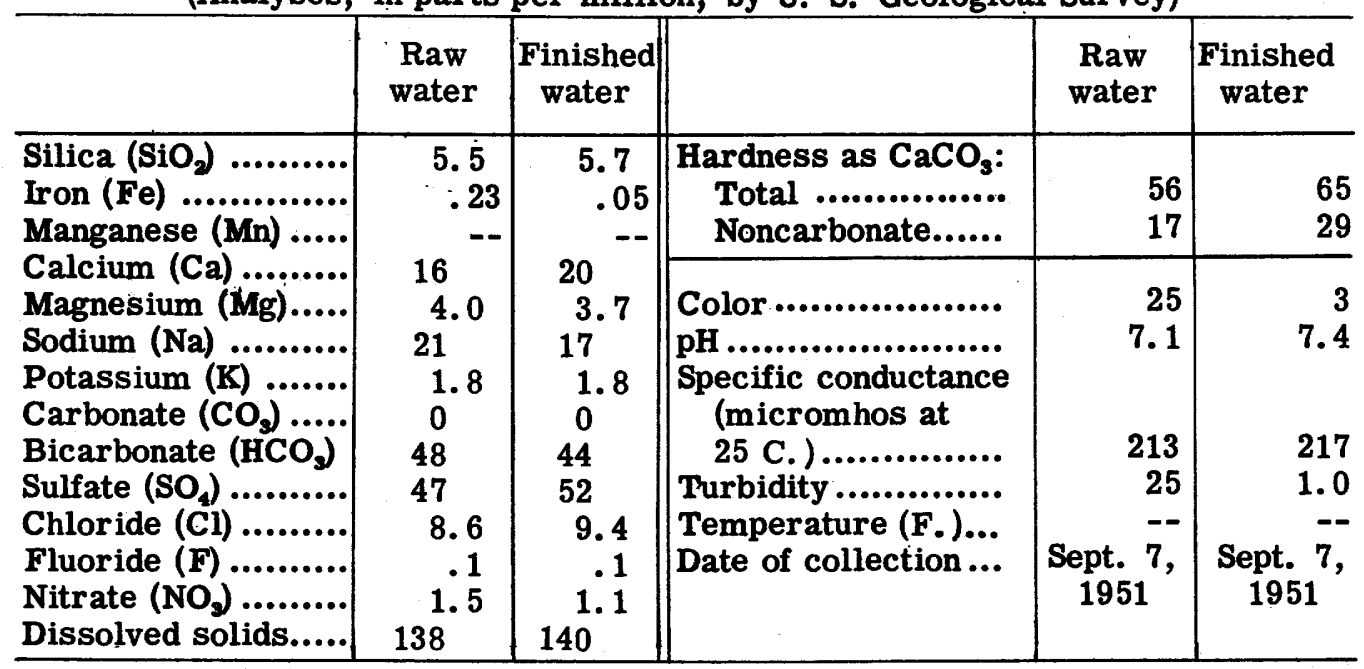

Regular determinations at treatment plant, 1950

\begin{tabular}{l|r|r|r|r|r|r|r|r|r|r|r|r}
\hline & \multicolumn{3}{|c|}{$\begin{array}{c}\text { Alkalinity } \\
\text { as } \begin{array}{c}\text { CaCO } \\
\text { (ppm) }\end{array}\end{array}$} & \multicolumn{3}{c|}{ pH } & \multicolumn{3}{c|}{$\begin{array}{c}\text { Hardness } \\
\text { as CaCO } \\
\text { (ppm) }\end{array}$} & \multicolumn{3}{c}{ Turbidity } \\
\cline { 2 - 12 } & Av & Max & Min & Av & Max & Min & Av & Max & Min & Av & Max & Min \\
\hline Raw water......... & -- & 35 & 18 & - & -- & -- & -- & -- & -- & -- & 350 & 30 \\
Finished water... & -- & 35 & 24 & - & 7.4 & 7.0 & -- & 60 & 30 & -- & -- & -- \\
\hline
\end{tabular}




\section{LYNCHBURG}

(Population, 47, 727)

Ownership: Municipal; also supplies a population of about 7,000 people outside the city limits, including Madison Heights Sanitary District in Amherst County. Total population supplied, about 55,000.

Source: Pedlar River impounded in Pedlar Lake about 23 miles northwest of the city. Auxiliary or emergency supply, James River.

Treatment: Coagulation with alum, sedimentation, sand filtration, chlorination, and fluoridation.

Rated capacity of treatment plant: $8,000,000 \mathrm{gpd}$.

Raw-water storage: $600,000,000$ gal.

Finished-water storage: 9,000,000 gal.

Water from Pedlar Lake flows by gravity through a 36 in. cast iron pipe to the treatment plant in the southwest section of the city. Finished water is stored in College Hill reservoir, Clay Street reservoir, and Fort Hill standpipe.

Distribution is by gravity flow.

\section{ANALYSES}

(Analyses, in parts per million, by U. S. Geological Survey)

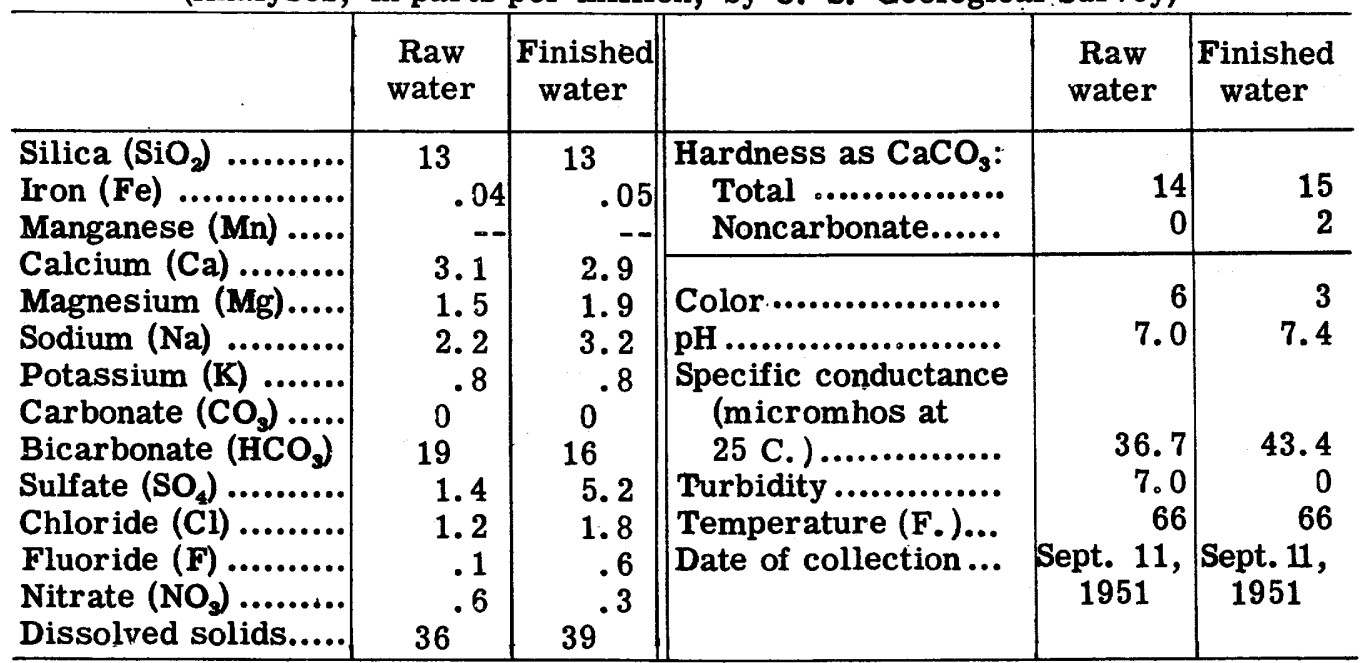

Regular determinations at treatment plant, 1950

\begin{tabular}{|c|c|c|c|c|c|c|c|c|c|c|c|c|}
\hline & \multicolumn{3}{|c|}{$\begin{array}{c}\text { Alkalinity } \\
\text { as CaCO } \\
\text { (ppm) }\end{array}$} & \multicolumn{3}{|c|}{$\mathrm{pH}$} & \multicolumn{3}{|c|}{$\begin{array}{l}\text { Hardness } \\
\text { as } \mathrm{CaCO} \\
\text { (ppm) }\end{array}$} & \multicolumn{3}{|c|}{ Turbidity } \\
\hline & Av & Max & Min & Av & $\operatorname{Max}$ & Min & Av & Max & Min & Av & Max & Min \\
\hline $\begin{array}{l}\text { Raw water.......... } \\
\text { Finished water }\end{array}$ & 11 & 14 & 9 & 6.5 & 6.9 & 6.2 & -- & -- & - & 9 & 32 & 5 \\
\hline Finished wa & 9 & 10 & 8 & 6.3 & 6.6 & 6.0 & -- & -- & - & 0 & 0 & 0 \\
\hline
\end{tabular}




\section{MART INSVILLE}

(Population, 17, 251)

Ownership: Municipal; also supplies about 1,000 people in suburban areas. Total population supplied, about 18,300 .

Source: Beaver Creek 60 percent of supply; Jones Creek 40 percent of supply.

Treatment: Prechlorination, coagulation with lime and alum, activated carbon, sedimentation, rapid sand filtration, postchlorination, and adjustment of $\mathrm{pH}$ with lime.

Rated capacity of treatment plant: 5,000,000 gpd.

Raw-water storage: 500,000 gal.

Finished-water storage: 2,000,000 gal.

\section{ANALYSES}

(Analyses, in parts per million, by U. S. Geological Survey)

\begin{tabular}{|c|c|c|c|}
\hline & $\begin{array}{c}\text { Beaver } \\
\text { Creek } \\
\text { (raw water) }\end{array}$ & $\begin{array}{c}\text { Jones } \\
\text { Creek } \\
\text { (raw water) }\end{array}$ & $\begin{array}{c}\text { Finished } \\
\text { water }\end{array}$ \\
\hline 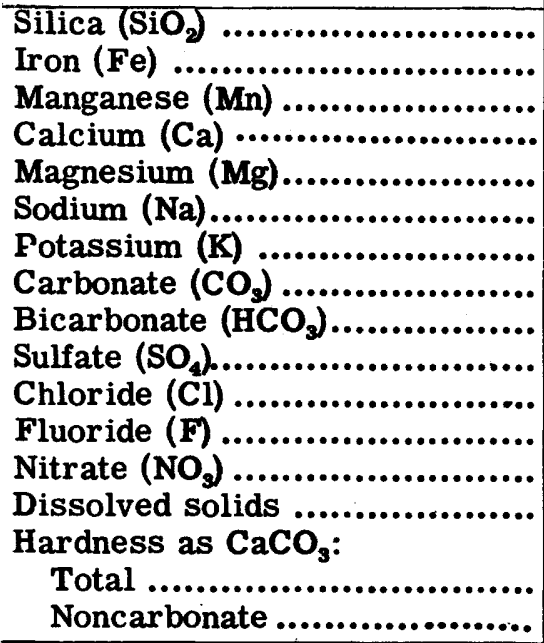 & $\begin{array}{l}20 \\
.82 \\
5.5 \\
2.5 \\
3.5 \\
1.3 \\
0 \\
34 \\
1.4 \\
2.9 \\
.2 \\
.3 \\
66 \\
24 \\
0\end{array}$ & \begin{tabular}{r|}
19 \\
.46 \\
$-\overline{-}$ \\
6.0 \\
1.7 \\
3.2 \\
1.0 \\
0 \\
28 \\
3.5 \\
1.4 \\
.1 \\
.3 \\
55 \\
22 \\
0
\end{tabular} & $\begin{array}{c}18 \\
.13 \\
12 \\
2.5 \\
3.5 \\
1.1 \\
0 \\
42 \\
9.7 \\
3.0 \\
.1 \\
.1 \\
76 \\
40 \\
6\end{array}$ \\
\hline 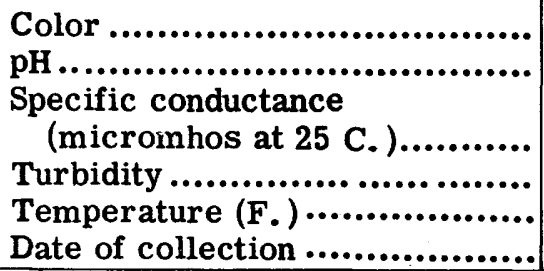 & $\begin{array}{r}35 \\
6.9 \\
65.7 \\
5.0 \\
65 \\
\text { Sept. } 11,1951\end{array}$ & $\begin{array}{r}30 \\
7.3 \\
49.8 \\
15 \\
64 \\
\text { Sept. } 11,1951\end{array}$ & $\begin{array}{r}0 \\
8.2 \\
98.4 \\
.5 \\
68 \\
\text { Sept. } 11,1951\end{array}$ \\
\hline
\end{tabular}

Regular determinations at treatment plant, 1950

\begin{tabular}{l|r|r|r|r|r|r|r|r|r|r|r|r}
\hline & \multicolumn{3}{|c|}{$\begin{array}{c}\text { Alkalinity } \\
\text { as CaCO } \\
\text { (ppm) }\end{array}$} & \multicolumn{3}{c|}{ pH } & \multicolumn{3}{c|}{$\begin{array}{c}\text { Hardness } \\
\text { as CaCO } \\
\text { (ppm) }\end{array}$} & \multicolumn{3}{c}{ Turbidity } \\
\cline { 2 - 13 } & Av & Max & Min & Av & Max & Min & Av & Max & Min & Av & Max & Min \\
\hline Raw water......... & 21 & 29 & 11 & 6.9 & 7.1 & 6.6 & -- & -- & -- & 70 & 2000 & 20 \\
Finished water... & 19 & 27 & 11 & 6.7 & 6.9 & 6.0 & -- & -- & -- & 0.5 & 0.5 & 0.5 \\
\hline
\end{tabular}




\section{NEWPORT NEWS}

(Population, 42,358)

Ownership: Municipal; also supplies Hampton, Hilton, Phoebus, and other communities in Elizabeth City, Warwick and York Counties. Total population supplied, about 150,000 .

Source: Chickahominy River 42 percent of supply; Lee Hall and Harwood Mill reservoirs 58 percent of supply. Skiffs Creek can be used in emergencies.

Treatment: Prechlorination, aeration, coagulation with alum, addition of carbon, chlorination, sedimentation, rapid sand filtration, postchlorination, and adjustment of $\mathrm{pH}$ with lime.

Rated capacity of treatment plants: 2 Lee Hall Reservoir plants: 8, 000, $000 \mathrm{gpd}$, and 6,000,000 mgd. Harwoòd Mill Reservoir plant: 6,000,000 mgd.

Raw-water storage: 1,754,000,000 gal.

Finished-water storage: Lee Hall underground reservoir, 3, 500,000 gal; concrete reservoir (in Newport News), 3,500,000 gal; 2 elevated tanks, 1, 000, 000 and $100,000 \mathrm{gal}$.

Lee Hall and Harwood Mill reservoirs store the headwaters of Warwick and Poquoson rivers, respectively. Water from the Chicahominy River is discharged from a 32 mile 18 in. pipeline into Harwood Mill reservoir. Combined waters from the Harwood Mill Reservoir are discharged into the Lee Hall Reservoir.

\section{ANALYSES}

(Analyses, in parts per million, by U. S. Geological Survey)

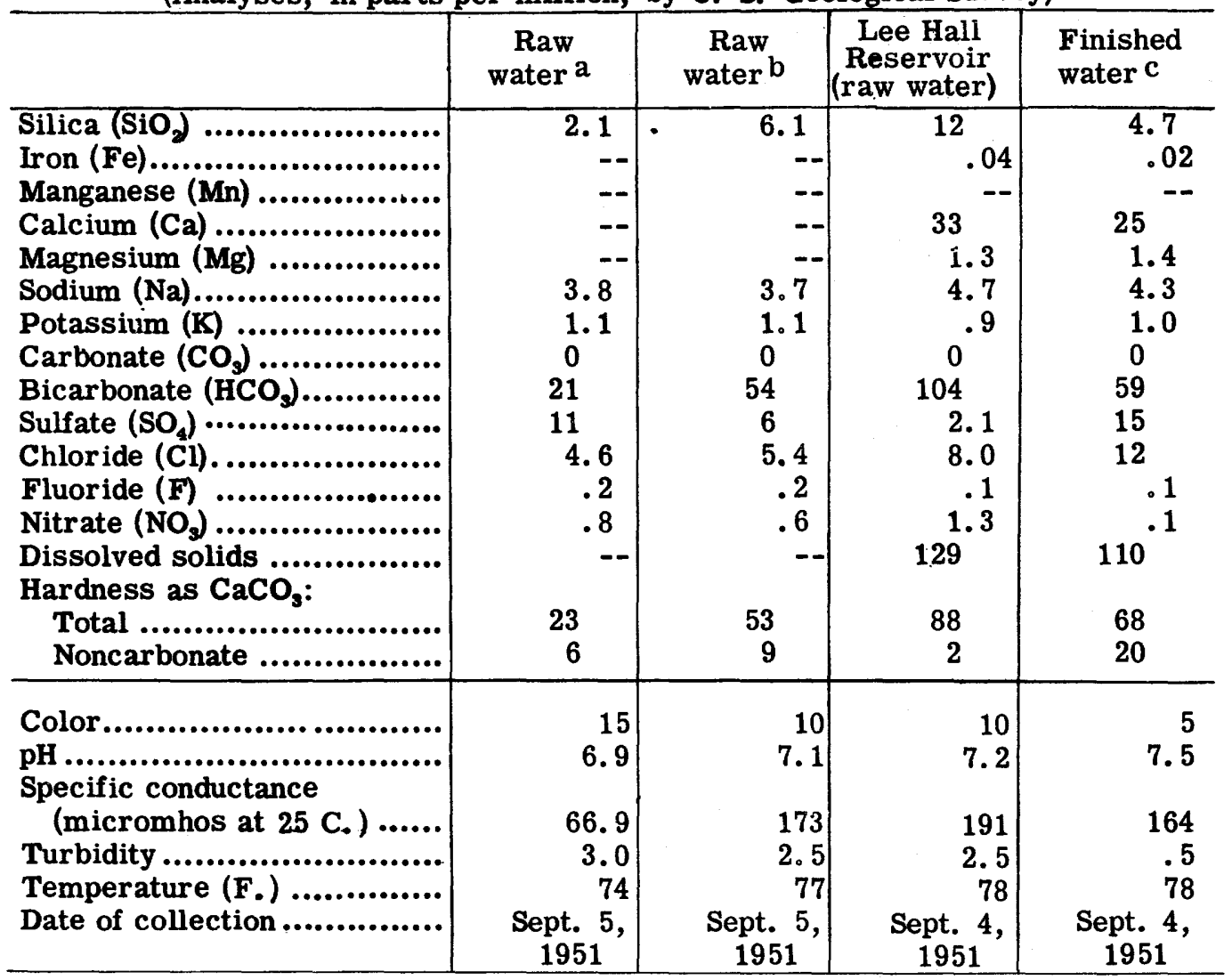

\footnotetext{
a Chickahominy River.

b Harwood Mill Reservoir.

c Supply System.
} 
NEWPORT NEWS--Continued

Regular determinations at treatment plant, 1950

\begin{tabular}{|c|c|c|c|c|c|c|c|c|c|c|c|c|}
\hline & \multicolumn{3}{|c|}{$\begin{array}{c}\text { Alkalinity } \\
\text { as CaCO } \\
\text { (ppm) }\end{array}$} & \multicolumn{3}{|c|}{$\mathrm{pH}$} & \multicolumn{3}{|c|}{$\begin{array}{l}\text { Hardness } \\
\text { as } \mathrm{CaCO} \\
\text { (ppm) }\end{array}$} & \multicolumn{3}{|c|}{ Turbidity } \\
\hline & Av & $\operatorname{Max}$ & Min & $A \nabla$ & Max & Min & Av & $\operatorname{Max}$ & Min & Av & $\operatorname{Max}$ & Min \\
\hline $\begin{array}{l}\text { Raw water......... } \\
\text { Finished water... }\end{array}$ & $\begin{array}{l}57 \\
53\end{array}$ & \begin{tabular}{|l|}
63 \\
59 \\
\end{tabular} & $\begin{array}{l}52 \\
49\end{array}$ & $\begin{array}{l}7.6 \\
8.0\end{array}$ & \begin{tabular}{|l|}
7.7 \\
8.0
\end{tabular} & \begin{tabular}{|l|}
7.5 \\
7.9
\end{tabular} & $\overline{93}$ & $\overline{113}$ & $\overline{73}$ & $\begin{array}{r}2.2 \\
.5\end{array}$ & \begin{tabular}{|r|}
2.3 \\
.5 \\
\end{tabular} & $\begin{array}{r}2.0 \\
.5\end{array}$ \\
\hline
\end{tabular}

\section{NORFOLK}

(Population, 213, 513)

Ownership: Municipal; also supplies Portlock, South Norfolk, Virginia Beach, other communities, suburban areas, and an unknown number of Army and Navy personnel. Total population supplied, about 300,000 .

Source: Two systems of impounding reservoirs (lakes): Lake Smith system comprised of a chain of reservoirs known as Lake Wright, Lake Taylor, Little Creek, Lake Lawson, Lake Smith and North Landing Lake about 5 miles northeast of the city, and for the most part in Princess Anne County; and Lake Prince system comprised of Lake Prince on Exchange Creek and Lake Burnt Mills, a subsidiary reservoir, in Nansemond County about 18 miles from the city. Auxiliary or emergency supply, Nottoway and Blackwater Rivers.

Treatment: (Both plants) prechlorination, coagulation with alum and lime, addition of activated carbon, bleaching clay when needed, sedimentation, rapid sand filtration, postchlorination, and adjustment of $\mathrm{pH}$ with lime.

Rated capacity of treatment plants: Moores Bridges plant, 24,000,000 gpd; 37th Street plant, 24,000,000 gpd.

Raw-water storage: 9,700,000,000 gal.

Finished-water storage: 20,000,000 gal.

Water from the Lake Smith supply system is treated at the Moores Bridges treatment plant located at the southern end of Lake Wright. Water from Lake Prince and Lake Burnt Mills is conducted in two parallel mains, interconnected at intervals, to the 37th Street treatment plant in Norfolk, where it is treated. The two parallel mains are also connected by a 48 in. concrete main to the Moores Bridges treatment plant, so that with this arrangement each of the three mains can deliver water to either plant. 
NORFOLK--Continued

ANALYSES

(Analyses, in parts per million, by U. S. Geological Survey)

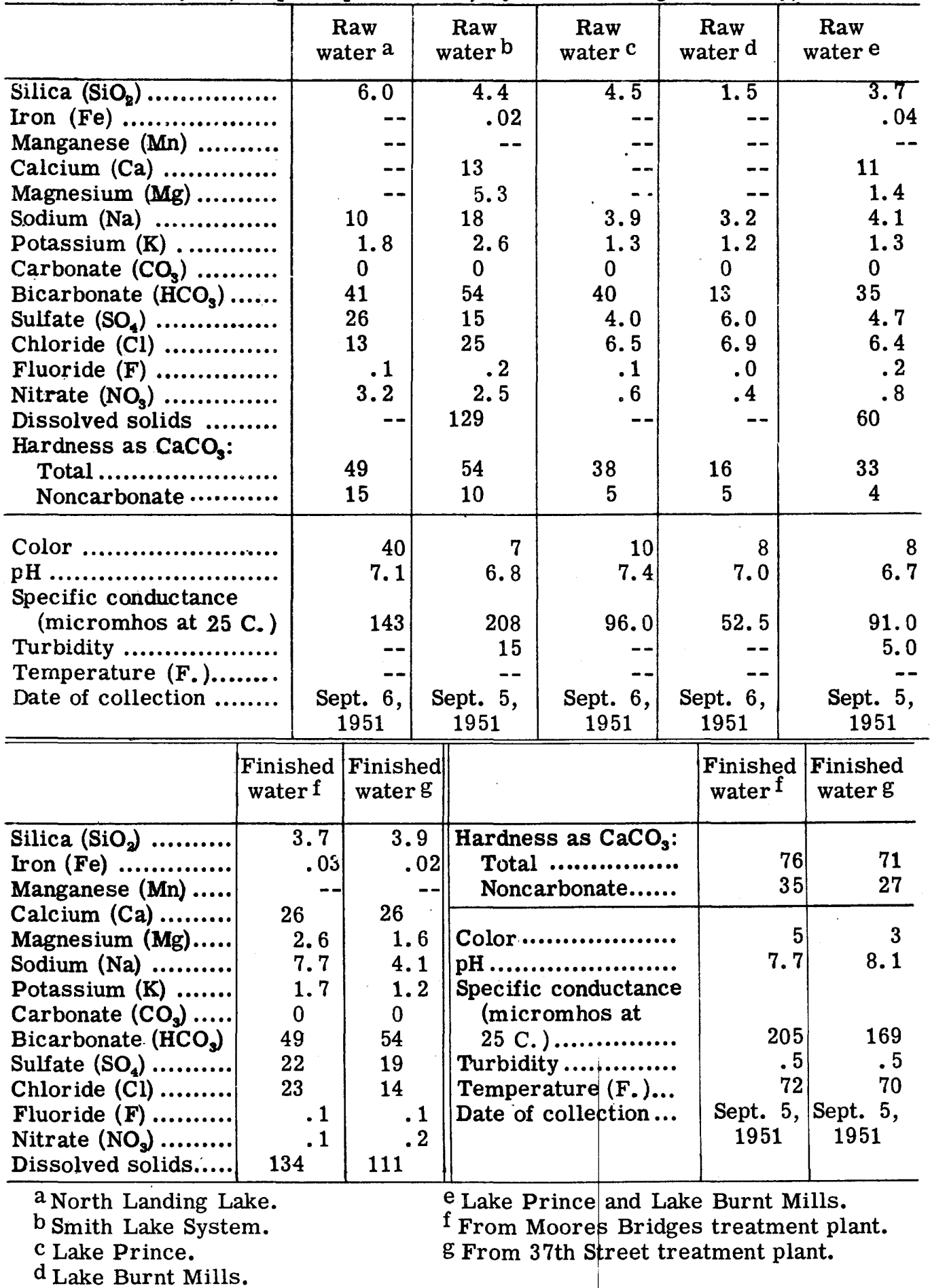


NORFOLK--Continued

Regular determinations at treatment plant, 1951

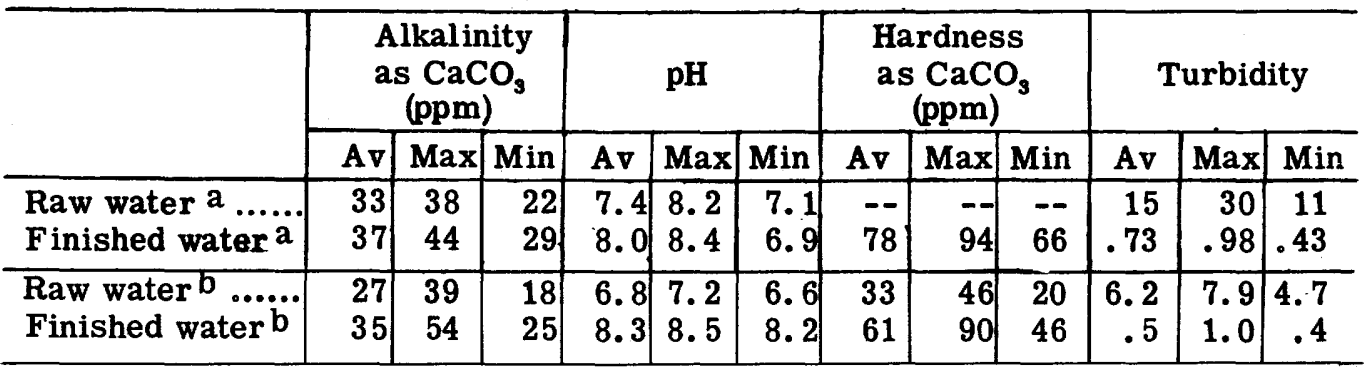

a Moores Bridges Plant.

b 37th Street Plant.

\section{PETERSBURG}

(Population, 35, 054)

Ownership: Municipal; also supplies suburban areas, other communities, and Fort Lee part of the time. Total population supplied, about 70,000.

Source: Appomattox River (through power canal owned by the Va. Electric \& Power Co.). Emergency supply, Wilcox Lake (Lieutenant Run impounded).

Treatment: Plain sedimentation, prechlorination, coagulation with alum and lime, sedimentation, addition of carbon, rapid sand filtration, postchlorination, and adjustment of $\mathrm{pH}$ with lime.

Rated capacity of treatment plant: 8,000,000 gpd.

Raw-water storage: 5,000,000 gal.

Finished-water storage: 3,240,000 gal.

The intake on the power canal is about 3 miles from the treatment plant, located within the city limits. Under normal operation conditions the flow of the water from the canal to the treatment plant is by gravity, but when the consumption exceeds a specified volume pumping becomes necessary. The city has prior right to $10,000,000 \mathrm{gpd}$. 
PETERSBURG--Continued

\section{ANALYSES}

(Analyses, in parts per million, by U. S. Geological Survey)

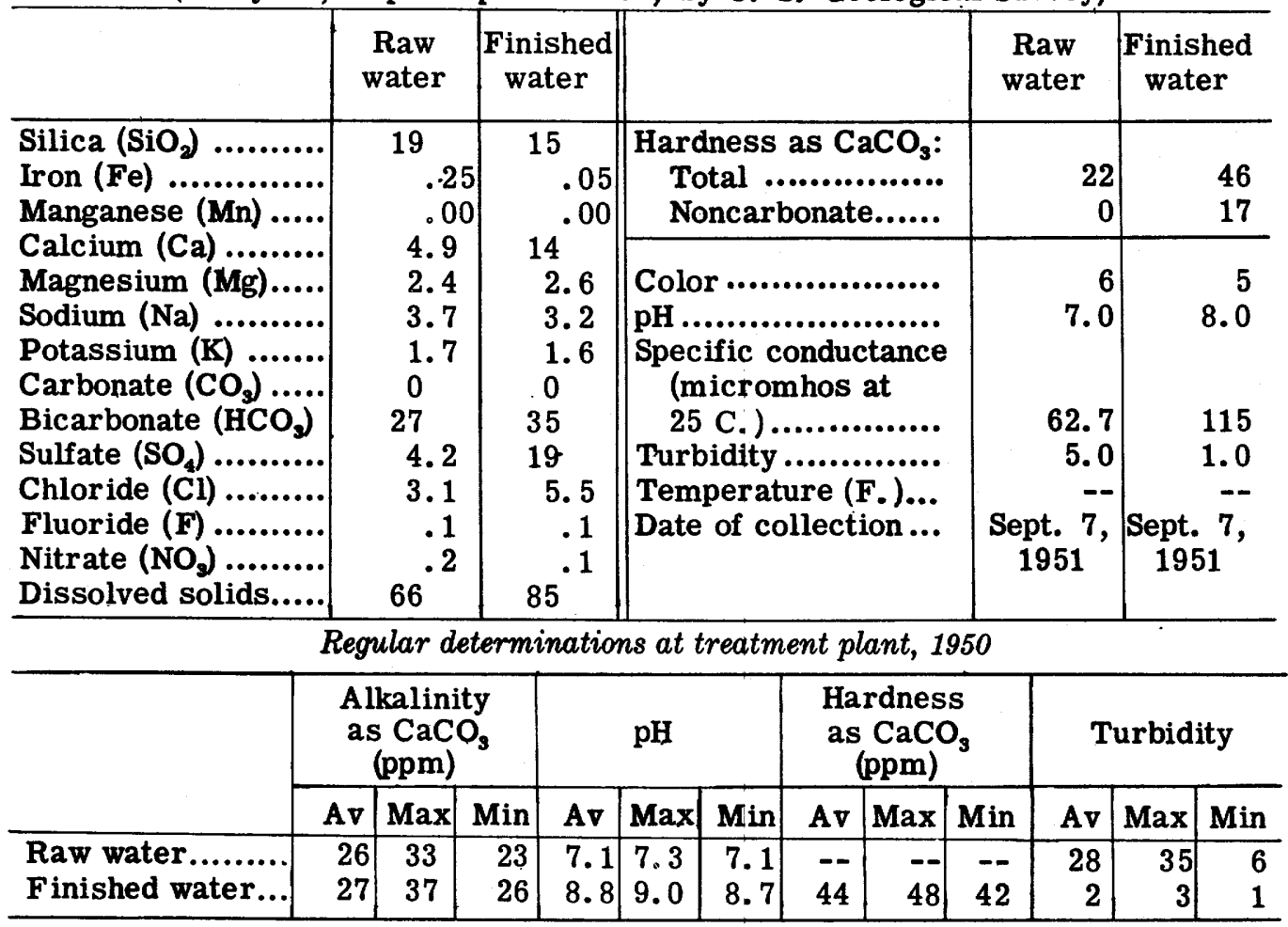


PORTSMOUTH

(Population, 80,039)

Ownership: Municipal; also supplies a population of about 50,000 in Norfolk Co., and 20,000 in the city of Suffolk and Nansemond Co. Total population supplied, about 150,000 .

Source: Lake Kilby 66 percent of supply; Lake Cahoon 34 percent of supply. Emergency supply, Nottoway River.

Treatment: Prechlorination, coagulation with lime and alum, sedimentation, activated carbon, rapid sand filtration, and addition of polyphosphate (Calgon) for corrosion control.

Rated capacity of treatment plant: $20,000,000$ gpd.

Raw-water storage: 2, 450,000,000 gal.

Finished-water storage: $11,000,000$ gal.

Lake Kilby is located just west of the corporate limits of Suffolk; Lake Cahoon is about 2 miles north of Lake Kilby in Nansemond County. Water is piped from Lake Cahoon to the treatment plant at the eastern end of Lake Kilby where the water from both sources is treated.

\section{ANALYSES}

(Analyses, in parts per million, by U. S. Geological Survey)

\begin{tabular}{|c|c|c|c|}
\hline$\cdot$ & $\begin{array}{r}\text { Lake Kilby } \\
\text { (raw water) }\end{array}$ & $\begin{array}{c}\text { Lake Cahoon } \\
\text { (raw water) }\end{array}$ & $\begin{array}{l}\text { Finished } \\
\text { water }\end{array}$ \\
\hline 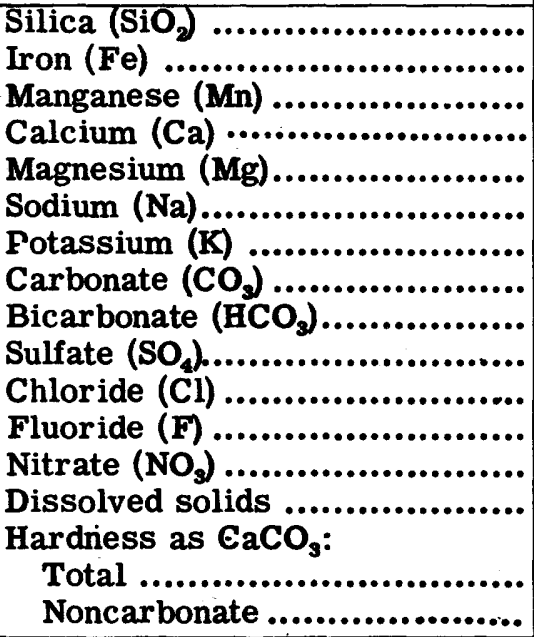 & \begin{tabular}{c|}
4.0 \\
.14 \\
5.9 \\
1.7 \\
6.1 \\
1.2 \\
0 \\
17 \\
7.0 \\
10 \\
.3 \\
.7 \\
60 \\
22 \\
8
\end{tabular} & $\begin{array}{c}3.7 \\
.04 \\
-- \\
5.2 \\
1.7 \\
4.7 \\
1.0 \\
0 \\
18 \\
5.2 \\
7.5 \\
.3 \\
.4 \\
49 \\
20 \\
5\end{array}$ & $\begin{array}{l}3.4 \\
.03 \\
14 \\
1 .- \\
1.4 \\
5.3 \\
1.1 \\
0 \\
22 \\
15 \\
14 \\
.0 \\
.1 \\
80^{.1} \\
41 \\
23\end{array}$ \\
\hline 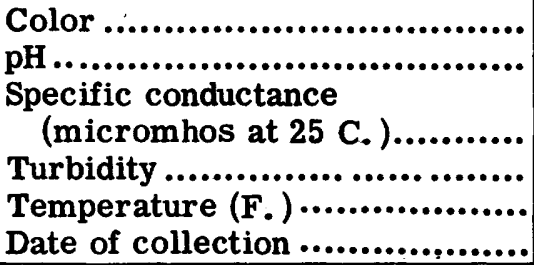 & $\begin{array}{r}33 \\
6.3 \\
77.7 \\
10 \\
69 \\
\text { Sept. } 6,1951\end{array}$ & $\begin{array}{r}15 \\
6.7 \\
64.5 \\
15 \\
71 \\
\text { Sept. } 6,1951 \\
\end{array}$ & $\begin{array}{r}3 \\
7.1 \\
\\
118 \\
1.0 \\
71 \\
\text { Sept. } 6,1951\end{array}$ \\
\hline
\end{tabular}

Regular determinations at treatment plant, 1949

\begin{tabular}{l|r|r|r|r|r|r|r|r|r|r|r|r|r}
\hline & \multicolumn{3}{|c|}{$\begin{array}{c}\text { Alkalinity } \\
\text { as } \begin{array}{c}\text { CaCO } \\
\text { (ppm) }\end{array}\end{array}$} & \multicolumn{3}{c|}{ pH } & \multicolumn{3}{c|}{$\begin{array}{c}\text { Hardness } \\
\text { as CaCO } \\
\text { (ppm) }\end{array}$} & \multicolumn{3}{c}{ Turbidity } \\
\cline { 2 - 13 } & Av & Max & Min & Av & Max & Min & Av & Max & Min & Av & Max & Min \\
\hline Raw water........ & 9 & 13 & 6 & 6.1 & 6.4 & 6.0 & -- & -- & --2 & 9 & 29 & 3 \\
Finished water... & 16 & 24 & 10 & 7.1 & 8.4 & 6.5 & 28 & 32 & 25 & 0 & 0 & 0 \\
\hline
\end{tabular}




\section{PULASKI}

(Population, 9, 202)

Ownership: Municipal; also supplies about 1,000 people outside the city limits. Total population supplied, about 10,200.

Source: Lake (impounded mountain stream) 97 percent of supply; Wardens Spring 3 percent of supply.

Treatment: Filtration and chlorination.

Rated capacity of treatment plant: 3,000,000 gpd.

Raw-water storage: 304,000,000 gal.

Finished-water storage: 1, 500,000 gal.

The Lake is about 5 miles west of town; Wardens Spring is about $1 \frac{1}{2}$ miles east of town.

\section{ANALYSES}

(Analyses, in parts per million, by U. S. Geological Survey)

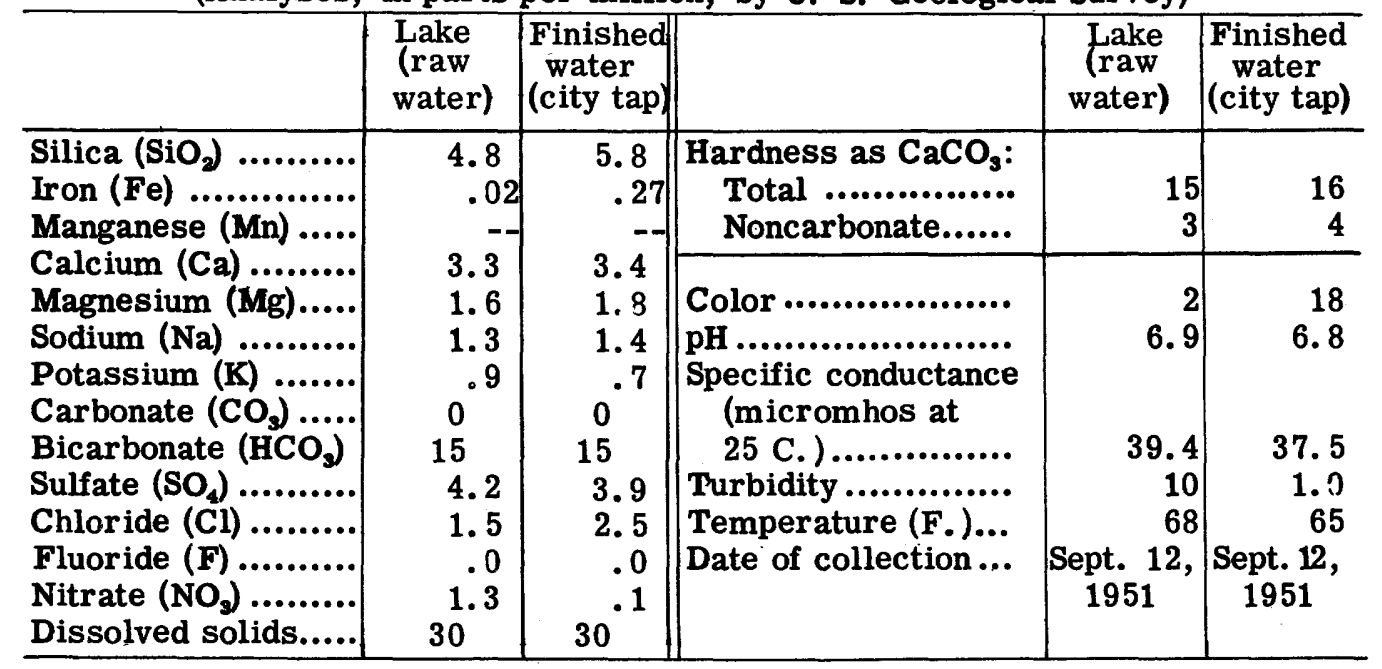

Regular determinations at treatment plant, 1952

\begin{tabular}{l|r|r|r|r|r|r|r|r|r|r|r|r}
\hline & \multicolumn{3}{|c|}{$\begin{array}{c}\text { Alkalinity } \\
\text { as CaCO } \\
\text { (ppm) }\end{array}$} & \multicolumn{3}{c|}{$\mathrm{pH}$} & \multicolumn{3}{c|}{$\begin{array}{c}\text { Hardness } \\
\text { as CaCO } \\
\text { (ppm) }\end{array}$} & \multicolumn{3}{c}{ Turbidity } \\
\cline { 2 - 13 } & Av & Max & Min & Av & Max & Min & Av & Max & Min & Av & Max & Min \\
\hline Raw water......... & 8 & 9 & 7 & 6.3 & 6.7 & 6.3 & 12 & 12 & 11 & 2 & 2 & 1 \\
Finished water... & 15 & 15 & 15 & 8.6 & 8.8 & 8.6 & 22 & 22 & 22 & 0 & 0 & 0 \\
\hline
\end{tabular}




\section{RICHMOND}

(Population, 230, 310)

Ownership: Municipal; also supplies a population of about 20,000 outside the city limits. Total population supplied, about 250,000 .

Source: James River. Gravity flow.

Treatment: Prechlorination, coagulation with alum (in summer) or ferrous sulfate (in winter), sedimentation, activated carbon, rapid sand filtration, postchlorination, ammoniation, adjustment of $\mathrm{pH}$ with lime, and addition of copper sulfate.

Rated capacity of treatment plant: $66,000,000$ gpd.

Raw-water storage: 170, 000,000 gal.

Finished-water storage: $58,000,000$ gal.

The treatment plant is on Douglasdale Road on the southwest edge of the city.

\section{ANALYSES}

(Analyses, in parts per million, by U. S. Geological Survey)

\begin{tabular}{|c|c|c|c|c|c|}
\hline & $\begin{array}{c}\text { Raw } \\
\text { water a }\end{array}$ & $\begin{array}{c}\text { Finished } \\
\text { water }\end{array}$ & & $\begin{array}{c}\text { Raw } \\
\text { water a }\end{array}$ & $\begin{array}{c}\text { Finished } \\
\text { water }\end{array}$ \\
\hline $\begin{array}{l}\text { Silica }\left(\mathrm{SiO}_{2}\right) \\
\text { Iron }(\mathrm{Fe}) \ldots \ldots \ldots \ldots \\
\text { Manganese }(\mathrm{Mn}) \ldots \ldots . . . . .\end{array}$ & $\begin{array}{l}7.5 \\
.03 \\
--\end{array}$ & $\begin{array}{c}8.5 \\
.02 \\
--\end{array}$ & $\begin{array}{r}\text { Hardness as } \mathrm{CaCO}_{3}: \\
\text { Total ................. } \\
\text { Noncarbonate...... }\end{array}$ & $\begin{array}{l}73 \\
26\end{array}$ & $\begin{array}{l}76 \\
47\end{array}$ \\
\hline 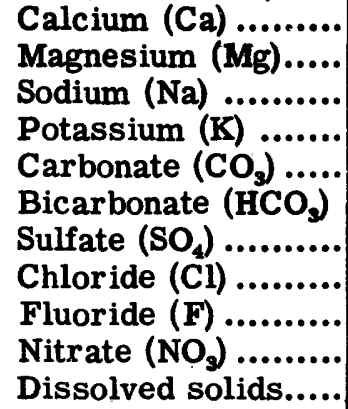 & $\begin{array}{l}21 \\
5.1 \\
12 \\
1.8 \\
0 \\
58 \\
37 \\
10 \\
.1 \\
133^{.6}\end{array}$ & $\begin{array}{l}23 \\
4.6 \\
9.9 \\
1.6 \\
0 \\
36 \\
48 \\
14 \\
.1 \\
.1\end{array}$ & $\begin{array}{l}\text { Color ................... } \\
\text { pH ......................... } \\
\text { Specific conductance } \\
\text { (micromhos at } \\
25 \text { C.) ................. } \\
\text { Turbidity ............. } \\
\text { Temperature (F.)... } \\
\text { Date of collection... }\end{array}$ & $\begin{array}{r}202 \\
\text { Sept.130, } \\
1951\end{array}$ & $\begin{array}{r}215 \\
.5 \\
77 \\
\text { Sept. } 3 \\
1951\end{array}$ \\
\hline
\end{tabular}

Regular determinations at treatment plant, 1950

\begin{tabular}{l|r|r|r|r|r|r|r|r|r|r|r|r|r}
\hline & \multicolumn{3}{|c|}{$\begin{array}{c}\text { Alkalinity } \\
\text { as CaCO, } \\
\text { (ppm) }\end{array}$} & \multicolumn{3}{c|}{ pH } & \multicolumn{3}{c|}{$\begin{array}{c}\text { Hardness } \\
\text { as CaCO, } \\
\text { (ppm) }\end{array}$} & \multicolumn{3}{c}{ Turbidity } \\
\cline { 2 - 14 } & Av & Max & Min & Av & Max & Min & Av & Max & Min & Av & Max & Min \\
\hline Raw water......... & 39 & 60 & 15 & 7.5 & 8.2 & 6.7 & -- & -- & -- & 30 & 250 & 5 \\
Finished water... & 30 & 48 & 12 & $\mathbf{8 . 8}$ & 9.3 & 8.4 & 65 & 80 & 50 & .1 & .2 & .1 \\
\hline
\end{tabular}

${ }^{\mathrm{a}}$ Composite of daily samples from the James River for the period Sept. 1 to $30,1951$. 


\section{ROANOKE \\ (Population, 91, 921)}

Ownership: Municipal; also supplies about 300 people outside the city limits. Total population supplied, about $92,200$.

Source: Impounding reservoirs (Carvin's Cove 40 percent, Beaver Creek and Falling Creek 15 percent), 55 percent of supply; Crystal Springs, 45 percent of supply.

Treatment: Carvin's Cove plant: Coagulation with ferric'sulfate, aeration, lime, sedimentation, rapid sand filtration, chlorination, and ammoniation. Falling Creek plant: coagulation with alum, sedimentation, pressure filtration, addition of lime and polyphosphate (Calgon), Crystal Springs water: chlorination.

Rated capacity of treatment plant: Carvin's Cove plant, 6,000,000 gpd; Falling Creek plant, 1, 500,000 gpd.

Raw-water storage: Carvin's Cove reservoir, 6,470,000,000 gal; Beaver Creek and Falling Creek reservoirs, 550,000,000 gal.

Finished-water storage: Carvin's Cove clear wells, 2,000,000 gal; Mill Mt. reservoir, 2, 000, 000 gal; City Farm reservoir, 2, 000,000 gal; Carroll Avenue. standpipe, 2, 000,000 gal; smaller elevated tanks and reservoirs, 3,000,000 gal.

Crystal Springs is within the city limits on the western slope of Mill Mountain, about $1 \frac{1}{2}$ miles from the center of the city. Carvin's Cove Reservoir is about 8 miles north of the city; Beaver Creek and Falling Creek reservoirs are about 7 miles northeast of the city.

Carvin's Cove treatment plant is about $3,000 \mathrm{ft}$ south of the impounding dam and the water flows to the treatment plant by gravity. Falling Creek treatment plant which treats water from both Beaver Creek and Falling Creek reservoirs is a short distance below Falling Creek dam.

The four principal finished-water storage reservoirs are strategically located in four sections of the city to aid in correcting unequal pressures due to over 200 $\mathrm{ft}$ differences in elevations in different sections of the city. Finished water from Carvin's Cove plant is conducted to both Mill Mt. reservoir which floats on the system and to other storage reservoirs in the city. Finished water from Falling Creek plant is served principally in the southeastern part of the city. 
ROANOKE--Continued

ANALYSES

(Analyses, in parts per million, by U. S. Geological Survey)

\begin{tabular}{|c|c|c|c|}
\hline & $\begin{array}{c}\text { Crystal } \\
\text { Springs } \\
\text { (raw water) }\end{array}$ & $\begin{array}{l}\text { Carvin's Cove } \\
\text { and Falling Cr. } \\
\text { raw water) }\end{array}$ & $\begin{array}{l}\text { Finished } \\
\text { water } \\
\text { (composite) }\end{array}$ \\
\hline Silica $\left(\mathrm{SiO}_{2}\right) \ldots \ldots \ldots \ldots \ldots \ldots \ldots \ldots$ & 11 & 5.4 & 10 \\
\hline Iron $(\mathrm{Fe}) \quad \ldots \ldots \ldots \ldots \ldots \ldots \ldots \ldots \ldots \ldots \ldots$ & .02 & .04 & .04 \\
\hline Manganese (Mn) .............. & & & \\
\hline 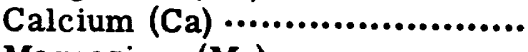 & 27 & 3.7 & 15 \\
\hline Magnesium (Mg).................... & 15 & 1.9 & 7.6 \\
\hline Sodium (Na) ........................... & 1.2 & 1.0 & 1.9 \\
\hline Fotassium (K) $\ldots \ldots \ldots \ldots \ldots \ldots \ldots \ldots \ldots$ & .5 & .5 & .4 \\
\hline Carbonate $\left(\mathrm{CO}_{3}\right) \ldots \ldots \ldots \ldots \ldots \ldots$ & & 0 & 0 \\
\hline Bicarbonate $\left(\mathrm{HCO}_{3}\right) \ldots \ldots \ldots \ldots \ldots$ & 151 & 18 & 82 \\
\hline 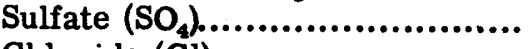 & 2.5 & 3.0 & 3.0 \\
\hline Chloride $(\mathrm{Cl}) \ldots \ldots \ldots \ldots \ldots \ldots \ldots \ldots \ldots$ & 1.0 & 1.5 & 2.2 \\
\hline Fluoride $(\mathbf{F}) \ldots \ldots \ldots \ldots \ldots \ldots \ldots \ldots \ldots \ldots$ & .0 & .1 & .1 \\
\hline Nitrate $\left(\mathrm{NO}_{3}\right) \ldots \ldots \ldots \ldots \ldots \ldots \ldots \ldots \ldots$ & 1.3 & .8 & .6 \\
\hline Dissolved solids ................... & 127 & 28 & 86 \\
\hline $\begin{array}{l}\text { Hardness as } \mathrm{CaCO}_{3}: \\
\text { Total } \ldots \ldots \ldots \ldots \ldots \ldots \ldots \ldots \ldots \ldots \ldots . \ldots \ldots\end{array}$ & 129 & 17 & 69 \\
\hline Noncarbonate ...................... & 5 & 2 & 2 \\
\hline Color ................... & & 3 & 2 \\
\hline pH .................... & 7.7 & 6.9 & 7.7 \\
\hline Specific conductance & & & \\
\hline (micromhos at 25 C. )........... & 228 & 39.4 & 139 \\
\hline Turbidity .............................. & & 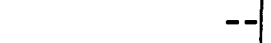 & -- \\
\hline Temperature (F. ) ............... & 62 & & 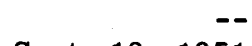 \\
\hline Date of collection.................. & Sept. 13,1951 & Sept. 13, 1951] & Sept. 13,1951 \\
\hline
\end{tabular}

Regular determinations at treatment plant, 1950

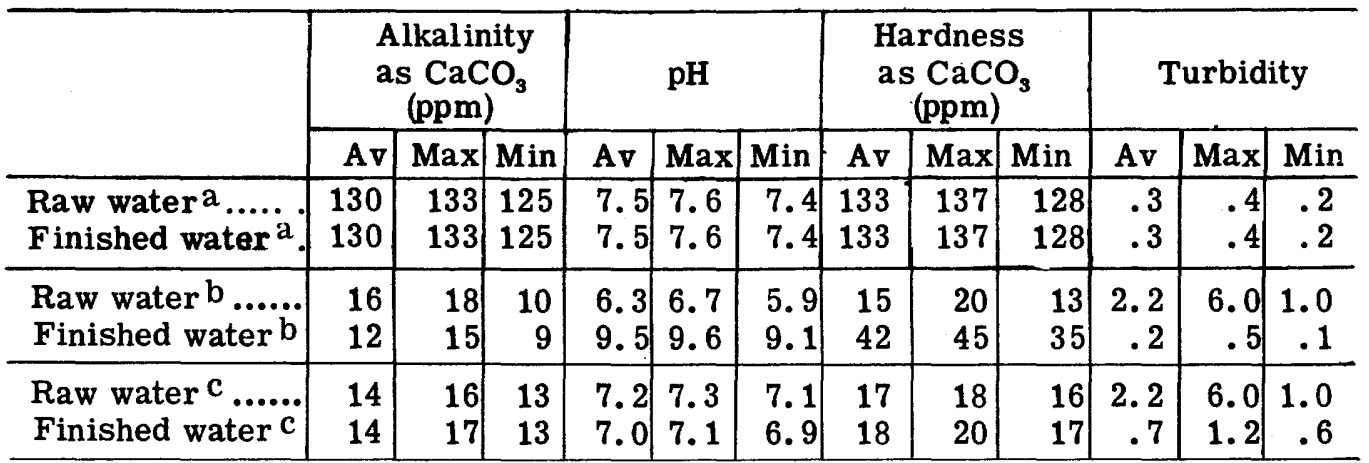

a Crystal Springs.

b Carvin's Cove.

c Falling Creek. 


\section{SOUTH NORFOLK}

(Population, 10,434)

Ownership: Supplied by Norfolk. (See Norfolk.)

STAUNTON

(Population, 19, 927)

Ownership: Municipal; also supplies about 300 people outside the city limits.

Total population supplied, about $20,200$.

Source: North River impounded. Auxiliary or emergency supply, Gardner's Spring about 3 miles west of the city.

Treatment: Prechlorination and addition of lime at N. River intake; coagulation with alum and silica (silicate + sulfuric acid), lime, sedimentation, rapid sand filtration, and postchlorination.

Rated capacity of treatment plant: $3,000,000$ gpd.

Raw-water storage: $125,000,000$ gal.

Finished-water storage: 7,000,000 gal.

The North River is impounded near Stokesville, about 35 miles northwest of Staunton. The water from the reservoir flows through a cast-iron pipe to the treatment plant, on Two-mile Hill about 2 miles west of the city. Finished water flows into the distribution system and to storage in the city.

\section{ANALYSES}

(Analyses, in parts per million, by U. S. Geological Survey)

\begin{tabular}{|c|c|c|c|}
\hline & $\begin{array}{l}\text { Gardner's } \\
\text { Spring } \\
\text { (raw water) }\end{array}$ & $\begin{array}{r}\text { North River } \\
\text { (raw water) }\end{array}$ & $\begin{array}{l}\text { Finished } \\
\text { water }\end{array}$ \\
\hline Silica $\left(\mathrm{SiO}_{2}\right) \ldots \ldots \ldots \ldots \ldots \ldots \ldots \ldots \ldots$ & -- & 4.1 & 5.4 \\
\hline Iron $(\mathrm{Fe})$ & -- & .04 & .02 \\
\hline Manganese (Mn) ..................... & -- & -- & - \\
\hline Calcium (Ca) $\ldots . . . . . . . . . . . . . . . . . . . .$. & -- & 1.6 & 10 \\
\hline Magnesium (Mg)..................... & -- & .9 & .9 \\
\hline 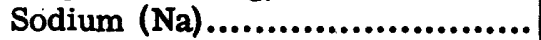 & -- & .8 & 1.7 \\
\hline Fotassium (K) ...................... & -- & .6 & .7 \\
\hline Carbonate $\left(\mathrm{CO}_{3}\right) \ldots \ldots \ldots \ldots \ldots \ldots$ & 0 & 0 & 0 \\
\hline Bicarbonate $\left(\mathrm{HCO}_{3}\right) \ldots \ldots \ldots \ldots \ldots . . . .$. & 294 & 8.0 & 25 \\
\hline Sulfate $\left(\mathrm{SO}_{4}\right) \ldots \ldots \ldots \ldots \ldots \ldots \ldots \ldots$ & 4 & 2.8 & 12 \\
\hline Chloride (Cl) $\ldots . . . \ldots \ldots \ldots \ldots \ldots \ldots \ldots$ & -- & .8 & 1.2 \\
\hline Fluoride $(F), \ldots \ldots \ldots \ldots \ldots \ldots \ldots \ldots$ & -- & .0 & .0 \\
\hline 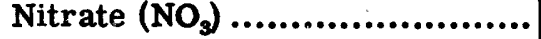 & 2.8 & .2 & .1 \\
\hline Dissolved solids ..................... & -- & 20 & 52 \\
\hline Hardness as $\mathrm{CaCO}_{3}$ : & & & \\
\hline Total $\ldots \ldots \ldots \ldots \ldots \ldots \ldots \ldots \ldots \ldots \ldots$ & 270 & 8 & 29 \\
\hline Noncarbonate ..................... & 29 & 1 & 8 \\
\hline Color ... & -- & & \\
\hline pH....... & 7.4 & 6.5 & 7.4 \\
\hline Specific conductance & & & \\
\hline (micromhos at 25 C. )..... & 428 & 21.1 & 71.3 \\
\hline Turbidity.....$\ldots \ldots \ldots \ldots \ldots \ldots \ldots$ & -- & & .5 \\
\hline Temperature (F.) $\ldots \ldots \ldots \ldots \ldots \ldots$ & 65 & & 69 \\
\hline Date of collection ................ & Sept. 11,1951 & Sept. 11, 1951 & Sept. 11, 1951 \\
\hline
\end{tabular}




\section{STAUNTON--Continued}

Regular determinations at treatment plant, Feb. to July, 1951

\begin{tabular}{l|r|r|r|r|r|r|r|r|r|r|r|r|r}
\hline & \multicolumn{3}{|c|}{$\begin{array}{c}\text { Alkalinity } \\
\text { as CaCO } \\
\text { (ppm) }\end{array}$} & \multicolumn{3}{|c|}{ pH } & \multicolumn{3}{c|}{$\begin{array}{c}\text { Hardness } \\
\text { as CaCO } \\
\text { (ppm) }\end{array}$} & \multicolumn{3}{c}{ Turbidity } \\
& Av & Max & Min & Av & Max & Min & Av & Max & Min & Av & Max & Min \\
\hline Raw water.......... & 14 & 28 & 0 & 7.8 & 8.2 & 7.4 & 16 & 20 & 10 & 3.2 & 6.9 & 1.1 \\
Finished water... & 22 & 28 & 10 & 8.4 & 9.0 & 7.6 & 28 & 32 & 22 & .6 & 1.1 & .2 \\
\hline
\end{tabular}

\section{SUFFOLK}

(Population, 12, 339)

Ownership: Supplied by Portsmouth. (See Portsmouth.)

WAYNESBORO

(Population, 12,357)

Ownership: Municipal; also supplies a small number of people outside the city

limits. Total population supplied, about 12,400 .

Source: Coyners Spring about 3 miles west of the city.

Treatment: Chlorination at the spring.

Rated capacity of treatment plant: $3,600,000 \mathrm{gpd}$.

Raw-water storage: None.

Finished-water storage: 2,000,000 gal.

The chlorinated water is pumped from the pump house adjacent to the spring basin to a 2,000,000 gal underground concrete reservoir, about $3 / 4$ mile southwest of the city, from which distribution to the city mains is by gravity.

\section{ANALYSES}

(Analyses, in parts per million, by U. S. Geological Survey)

\begin{tabular}{|c|c|c|c|c|c|}
\hline & $\begin{array}{l}\text { Raw } \\
\text { water }\end{array}$ & $\begin{array}{c}\text { Finished } \\
\text { water }\end{array}$ & & $\begin{array}{c}\text { Raw } \\
\text { water }\end{array}$ & $\begin{array}{l}\text { Finished } \\
\text { water }\end{array}$ \\
\hline $\begin{array}{l}\text { Silica }\left(\mathrm{SiO}_{2}\right) \ldots \ldots \ldots \ldots \\
\text { Iron }(\mathrm{Fe}) \ldots \ldots \ldots \ldots . . \\
\text { Manganese }(\mathrm{Mn}) \ldots \ldots\end{array}$ & \multirow{11}{*}{$\begin{array}{c}10 \\
.04 \\
19 \\
- \\
9.5 \\
.7 \\
1.2 \\
0 \\
96 \\
9.5 \\
.6 \\
.1 \\
.7 \\
96\end{array}$} & \multirow[t]{2}{*}{$\begin{array}{l}10^{-0} \\
19^{-}\end{array}$} & $\begin{array}{r}\text { Hardness as } \mathrm{CaCO}_{3}: \\
\text { Total .................. } \\
\text { Noncarbonate...... }\end{array}$ & $\begin{array}{r}86 \\
8\end{array}$ & $\begin{array}{r}86 \\
8\end{array}$ \\
\hline $\begin{array}{l}\text { Calcium }(\mathrm{Ca}) \ldots . . . . \\
\text { Magnesium }(\mathrm{Mg}) . . .\end{array}$ & & & \multirow{10}{*}{$\begin{array}{c}\text { Color ................... } \\
\text { pH .......................... } \\
\text { Specific conductance } \\
\text { (micromhos at } \\
\text { Turbidity .................. } \\
\text { Temperature (F.)... } \\
\text { Date of collection... }\end{array}$} & \multirow{4}{*}{$\begin{array}{r}5 \\
7.6\end{array}$} & \multirow{3}{*}{$\begin{array}{r}2 \\
7.6\end{array}$} \\
\hline Sodium (Na) ........... & & .7 & & & \\
\hline Potassium (K) ....... & & 1.2 & & & \\
\hline Carbonate $\left(\mathrm{CO}_{3}\right) \ldots .$. & & 0 & & & \\
\hline Bicarbonate $\left(\mathrm{HCO}_{3}\right)$ & & 96 & & 170 & 170 \\
\hline Sulfate $\left(\mathrm{SO}_{4}\right) \ldots \ldots \ldots \ldots$ & & 9.4 & & 0 & 0 \\
\hline Chloride $(\mathrm{Cl}) \ldots \ldots \ldots \ldots$ & & 1.0 & & 57 & 57 \\
\hline Fluoride (F) .......... & & .1 & & Sept.11, & Sept.11, \\
\hline Nitrate $\left(\mathrm{NO}_{3}\right) \ldots \ldots \ldots$ & & .9 & & 1951 & 1951 \\
\hline Dissolved solids..... & & 96 & & & \\
\hline
\end{tabular}




\section{WINCHESTER \\ (Population, 13, 841)}

Ownership: Municipal; also supplies about 2,000 people outside the city limits. Total population supplied, about 15,800 .

Source: Four limestone springs: Shawnee, Rouss, Fay's, and Old Town. Shawnee Spring is in the southeast part of the city; Rouss Spring, the main source of supply, about 1 mile southeast of the center of the city; Fay's Spring, 3 miles northeast of the city; and Old Town Spring, at the northwest side of the city.

Treatment: Superchlorination and dechlorination. Shawnee Spring water: coagulation with alum, sedimentation, rapid sand filtration, and chlorination.

Rated capacity of treatment plant: (Shawnee Spring plant only) 1,000,000 gpd.

Raw-water storage: None。

Finished-water storage: 3,200,000 gal.

Finished water from the spring basins is pumped into the distribution system and to storage.

\section{ANALYSES}

(Analyses, in parts per million, by U. S. Geological Survey)

\begin{tabular}{|c|c|c|c|c|c|}
\hline & $\begin{array}{c}\text { Shawnee } \\
\text { Spring } \\
\text { (raw water) }\end{array}$ & $\begin{array}{c}\text { Rouss } \\
\text { Spring } \\
\text { (raw water }\end{array}$ & $\begin{array}{c}\text { Fay's } \\
\text { Spring } \\
\text { (raw water) }\end{array}$ & $\begin{array}{c}\text { Old Town } \\
\text { Spring } \\
\text { (raw water) }\end{array}$ & $\begin{array}{c}\text { Finished } \\
\text { water } \\
\text { (city hall tap) }\end{array}$ \\
\hline Silica $\left(\mathrm{SiO}_{\mathrm{z}}\right) \ldots \ldots \ldots \ldots \ldots$ & -- & -- & -- & -- & 11 \\
\hline Iron $(\mathrm{Fe}) \ldots \ldots \ldots \ldots \ldots \ldots$ & -- & -- & -- & -- & .04 \\
\hline Manganese $(\mathbf{M n})$........... & -- & -- & -- & -- & -- \\
\hline Calcium (Ca) ............... & -- & -- & -- & -- & 87 \\
\hline Magnesium (Mg) ........... & -- & -- & -- & -- & 19 \\
\hline Sodium (Na) ................ & -- & -- & -- & -- & 3.4 \\
\hline Potassium (K) ............ & -- & - & -- & -- & 1.9 \\
\hline Carbonate $\left(\mathrm{CO}_{3}\right) \ldots \ldots \ldots . .$. & 0 & 0 & 0 & 0 & 6 \\
\hline Bicarbonate $\left(\mathrm{HCO}_{3}\right) \ldots . .$. & 387 & 368 & 322 & 272 & 310 \\
\hline Sulfate $\left(\mathrm{SO}_{4}\right) \ldots \ldots \ldots \ldots \ldots$ & 34 & 15 & 18 & 14 & 22 \\
\hline Chloride (Cl) .............. & -- & -- & -- & -- & 6.5 \\
\hline Fluoride $(F), \ldots \ldots \ldots \ldots \ldots$ & -- & -- & -- & -- & .1 \\
\hline Nitrate $\left(\mathrm{NO}_{3}\right) \ldots \ldots \ldots \ldots \ldots$ & 8.8 & 8.6 & 8.6 & 6.3 & 9.8 \\
\hline Dissolved solids $\ldots . . . . .$. & -- & -- & -- & -- & 358 \\
\hline Hardness as $\mathrm{CaCO}_{3}$ : & & & & & \\
\hline Total ....................... & 424 & 332 & 328 & 316 & 295 \\
\hline Noncarbonate ............ & 107 & 30 & 62 & 93 & 31 \\
\hline 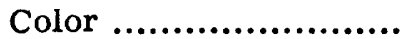 & -- & -- & -- & -- & 4 \\
\hline pH & 7.3 & 7.5 & 7.5 & 7.3 & 7.3 \\
\hline Specific conductance & & & & & \\
\hline (micromhos at $25 \mathrm{C}$. ) & 770 & 534 & 534 & 501 & 541 \\
\hline Turbidity & -- & -- & -- & -- & 1.0 \\
\hline Temperature (F.)........ & 58 & 58 & 61 & 57 & 66 \\
\hline Date of collection ......... & $\begin{array}{c}\text { Sept. } 12, \\
1951\end{array}$ & $\begin{array}{c}\text { Sept. } 12, \\
1951\end{array}$ & $\begin{array}{c}\text { Sept. } 12, \\
1951\end{array}$ & $\begin{array}{c}\text { Sept. } 12, \\
1951\end{array}$ & $\begin{array}{l}\text { Sept. 12, } \\
1951\end{array}$ \\
\hline
\end{tabular}




\section{BECKLEY}

(Population, 19, 397)

Ownership: Municipal; supplies also Mabscott, Sophia, and about 11,800 people out side the city limits. Total population supplied, about 34,300 .

Source: Glade Creek, impounded.

Treatment: Aeration, prechlorination, coagulation with alum and lime, sedimentation, rapid sand filtration, postchlorination, ammoniation, addition of Calgon for corrosion control.

Rated capacity of treatment plant: $2,300,000 \mathrm{gpd}$.

Raw-water storage: $565,000,000$ gal.

Finished-water storage: 3,835, 000 gal.

\section{ANALYSIS}

(Analysis, in parts per million, by U. S. Geological Survey)

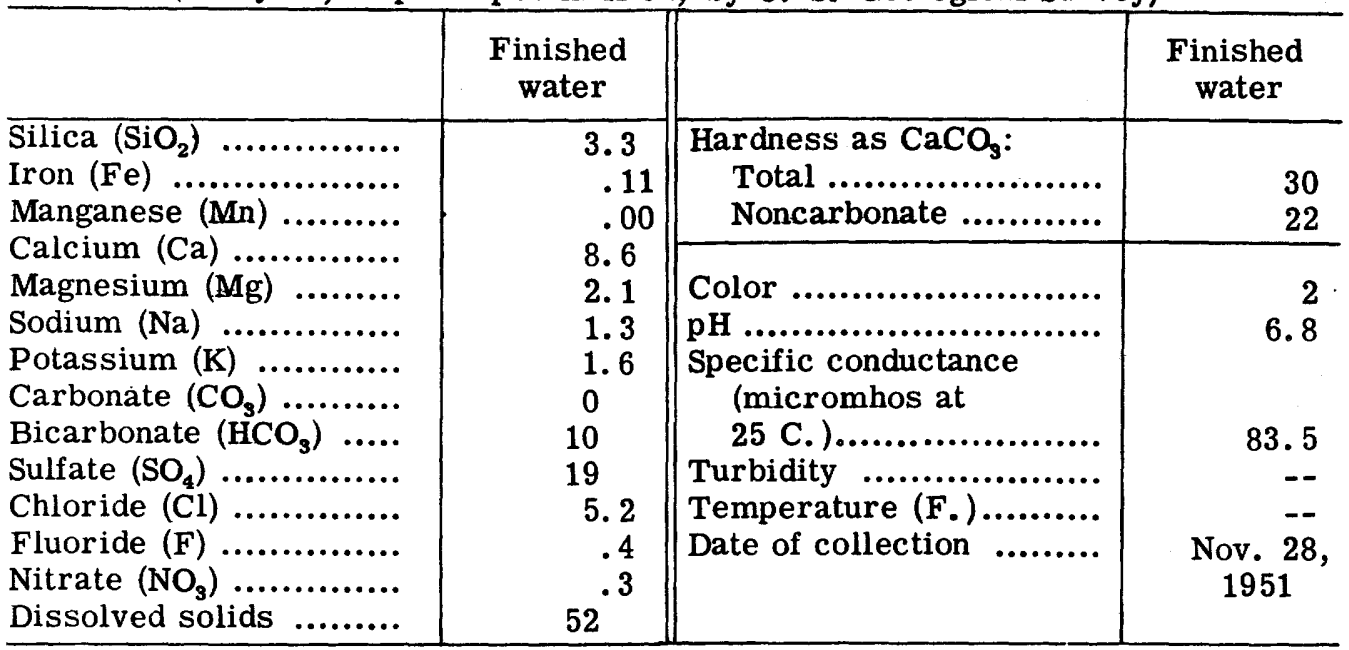

Regular determinations at treatment plant, 1950

\begin{tabular}{|c|c|c|c|c|c|c|c|c|c|c|c|c|}
\hline & \multicolumn{3}{|c|}{$\begin{array}{c}\text { Alkalinity } \\
\text { as } \mathrm{CaCO} \\
\text { (ppm) }\end{array}$} & \multicolumn{3}{|c|}{$\mathrm{pH}$} & \multicolumn{3}{|c|}{$\begin{array}{c}\text { Hardness } \\
\text { as } \mathrm{CaCO}_{3} \\
\text { (ppm) }\end{array}$} & \multicolumn{3}{|c|}{ Turbidity } \\
\hline & Av & $\operatorname{Max}$ & Min & Av & $\operatorname{Max}$ & Min & Av & $\operatorname{Max}$ & Min & Av & $\operatorname{Max}$ & Min \\
\hline $\begin{array}{l}\text { Raw water......... } \\
\text { Finished water... }\end{array}$ & $\begin{array}{r}8 \\
12\end{array}$ & $\begin{array}{l}14 \\
20\end{array}$ & \begin{tabular}{|l|}
3 \\
5 \\
\end{tabular} & $\begin{array}{l}6.3 \\
7.4\end{array}$ & $\begin{array}{l}7.1 \\
9.6\end{array}$ & $\begin{array}{l}6.1 \\
5.9\end{array}$ & $\begin{array}{l}16 \\
34\end{array}$ & $\begin{array}{l}22 \\
48 \\
\end{array}$ & $\begin{array}{r}8 \\
14\end{array}$ & $=$ & $\begin{array}{r}60 \\
0\end{array}$ & $\begin{array}{r}0.1 \\
0\end{array}$ \\
\hline
\end{tabular}




\section{BLUEFIELD}

(Population, 21, 506)

Ownership: West Virginia Water Service Company; supplies also about 2,000 people outside the city limits. Total population supplied, about 23,500.

Source: 22 springs and surface run-off feeding 2 reservoirs impounded by Ada Dam and Horton Dam. Auxiliary supply from Beaver Pond and Bailey Springs.

Treatment: Coagulation with alum, sedimentation, rapid sand filtration, and chlorination.

Rated capacity of treatment plant: 2, 000, 000 gpd.

Raw-water storage: 200, 000, 000 gal.

Finished-water storage: 2, 000, 000 gal.

\section{ANALYSIS}

(Analysis, in parts per million, by U. S. Geological Survey)

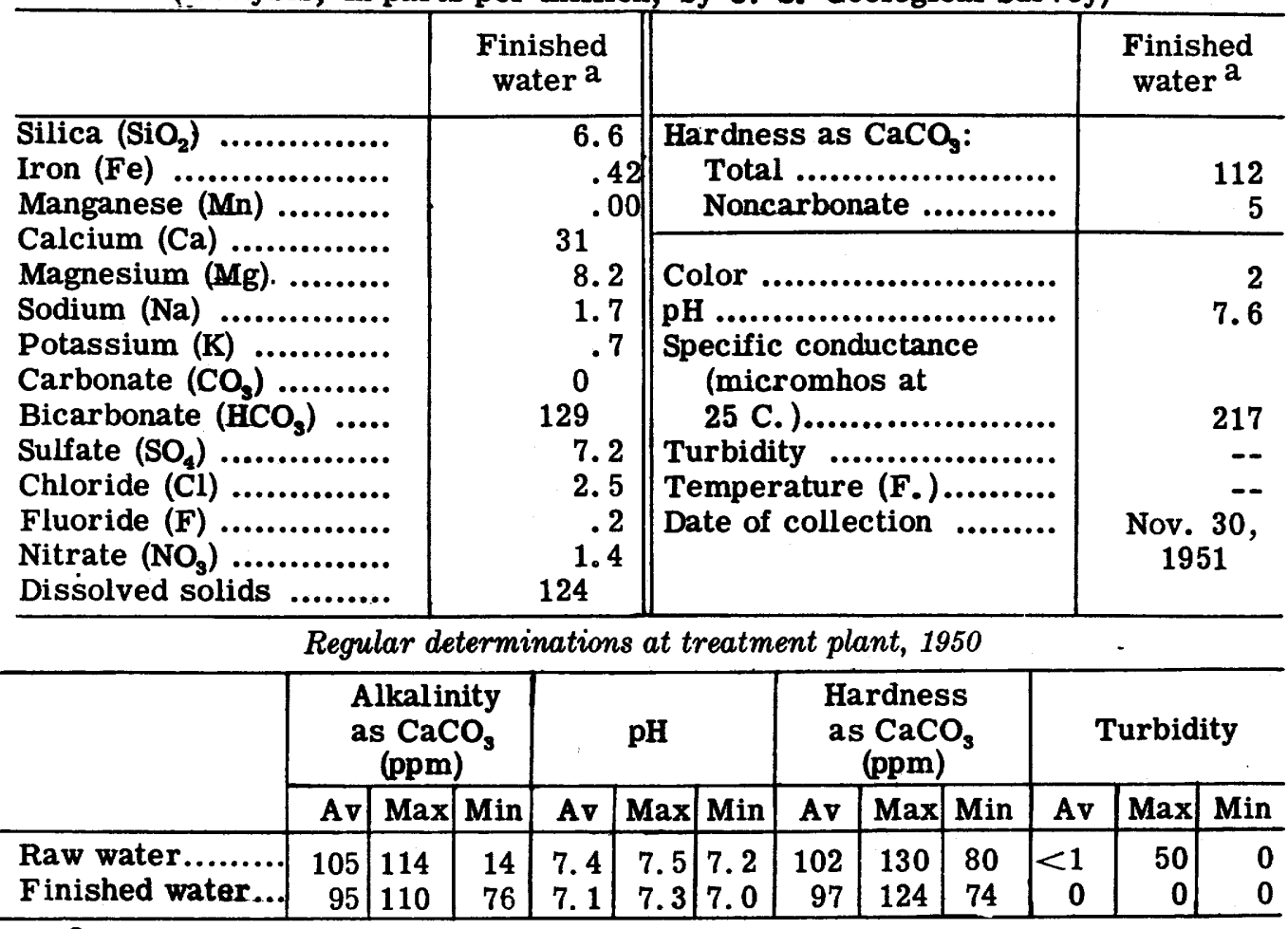

${ }^{a}$ All sources. 


\section{CHARLESTON \\ (Population, 73, 501)}

Ownership: West Virginia Water Service Company; supplies also South Charleston, and about 13, 900 people outside the city limits. The system is interconnected with Belle, Nitro, and St. Albans. Total population supplied, about 104, 000 .

Source: Elk River.

Treatment: Anhydrous ammonia, carbon, coagulation with alum and at times lime, aeration at times by air diffusion, chlorination beyond break-point, sedimentation, rapid sand filtration, and final adjustment of $\mathrm{pH}$ with lime.

Rated capacity of treatment plant: 20,000,000 gpd.

Raw-water storage: None..

Finished-water storage: 8, 850, 000 gal.

\section{ANALYSIS}

(Analysis, in parts per million, by U. S. Geological Survey)

\begin{tabular}{|c|c|c|c|}
\hline & $\begin{array}{l}\text { Finished } \\
\text { water }\end{array}$ & & $\begin{array}{l}\text { Finished } \\
\text { water }\end{array}$ \\
\hline $\begin{array}{l}\text { Silica }\left(\mathrm{SiO}_{2}\right) \\
\text { Iron }(\mathrm{Fe}) \quad \ldots \ldots \ldots \ldots \ldots \ldots \\
\text { Manganese }(\mathrm{Mn}) \ldots \ldots \ldots \ldots \ldots\end{array}$ & $\begin{array}{l}4.4 \\
.10 \\
.00\end{array}$ & $\begin{array}{l}\text { Hardness as } \mathrm{CaCO}_{3}: \\
\text { Total ....................... } \\
\text { Noncarbonate ........... }\end{array}$ & $\begin{array}{l}32 \\
17 \\
\end{array}$ \\
\hline 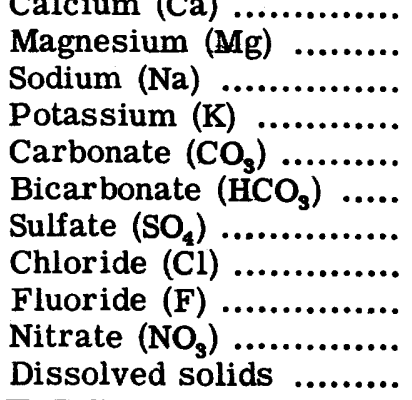 & $\begin{array}{l}9.6 \\
2.0 \\
2.3 \\
1.7 \\
0 \\
19 \\
15 \\
6.2 \\
.0 \\
1.8 \\
52\end{array}$ & 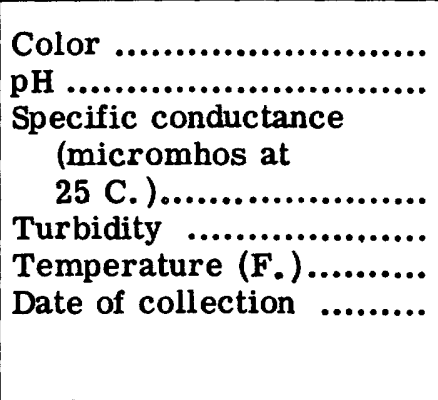 & $\begin{array}{r}89.4 \\
-- \\
-- \\
\text { Dec: } 27, \\
1951\end{array}$ \\
\hline
\end{tabular}

Regular determinations at treatment plant, 1950

\begin{tabular}{|c|c|c|c|c|c|c|c|c|c|c|c|c|}
\hline & \multicolumn{3}{|c|}{$\begin{array}{l}\text { Alkalinity } \\
\text { as CaCO } \\
\text { (ppm) }\end{array}$} & \multicolumn{3}{|c|}{ pH } & \multicolumn{3}{|c|}{$\begin{array}{c}\text { Hardness } \\
\text { as CaCO } \\
\text { (ppm) }\end{array}$} & \multicolumn{3}{|c|}{ Turbidity } \\
\hline & Av & $\operatorname{Max}$ & Min & Av & $\operatorname{Max}$ & Min & Av & $\operatorname{Max}$ & Min & Av & $\operatorname{Max}$ & Min \\
\hline $\begin{array}{l}\text { Raw water......... } \\
\text { Finished water.. }\end{array}$ & $\begin{array}{l}14 \\
21\end{array}$ & $\begin{array}{l}25 \\
36\end{array}$ & $\begin{array}{r}8 \\
13\end{array}$ & $\begin{array}{l}6.5 \\
9.0\end{array}$ & $\begin{array}{l}6.9 \\
9.6\end{array}$ & $\begin{array}{l}6.1 \\
8.4\end{array}$ & $\begin{array}{l}23 \\
37\end{array}$ & \begin{tabular}{l|}
42 \\
60
\end{tabular} & $\begin{array}{l}14 \\
22\end{array}$ & $\begin{array}{r}56 \\
>0\end{array}$ & $\begin{array}{r}1000 \\
0\end{array}$ & $\begin{array}{r}1 \\
>0\end{array}$ \\
\hline
\end{tabular}


CLARKSBURG

(Population, 32, 014)

Ownership: Municipal; supplies also Nutter Fort, Stonewood, Summit Park, and about 1,490 people outside the city limits. Total population supplied, about 38,400 .

Source: West Fork River impounded by a series of low dams.

Treatment: Coagulation with alum and lime, activated carbon, adjustment of $\mathrm{pH}$ to about 8.3 with lime, sedimentation, rapid sand filtration, postchlorination at times, softening by cation exchange at times.

Rated capacity of treatment plant: $9,000,000$ gpd.

Raw-water storage: $440,000,000$ gal

Finished-water storage: 3,064,000 gal.

\section{ANALYSIS}

(Analysis, in parts per million, by U. S. Geological Survey)

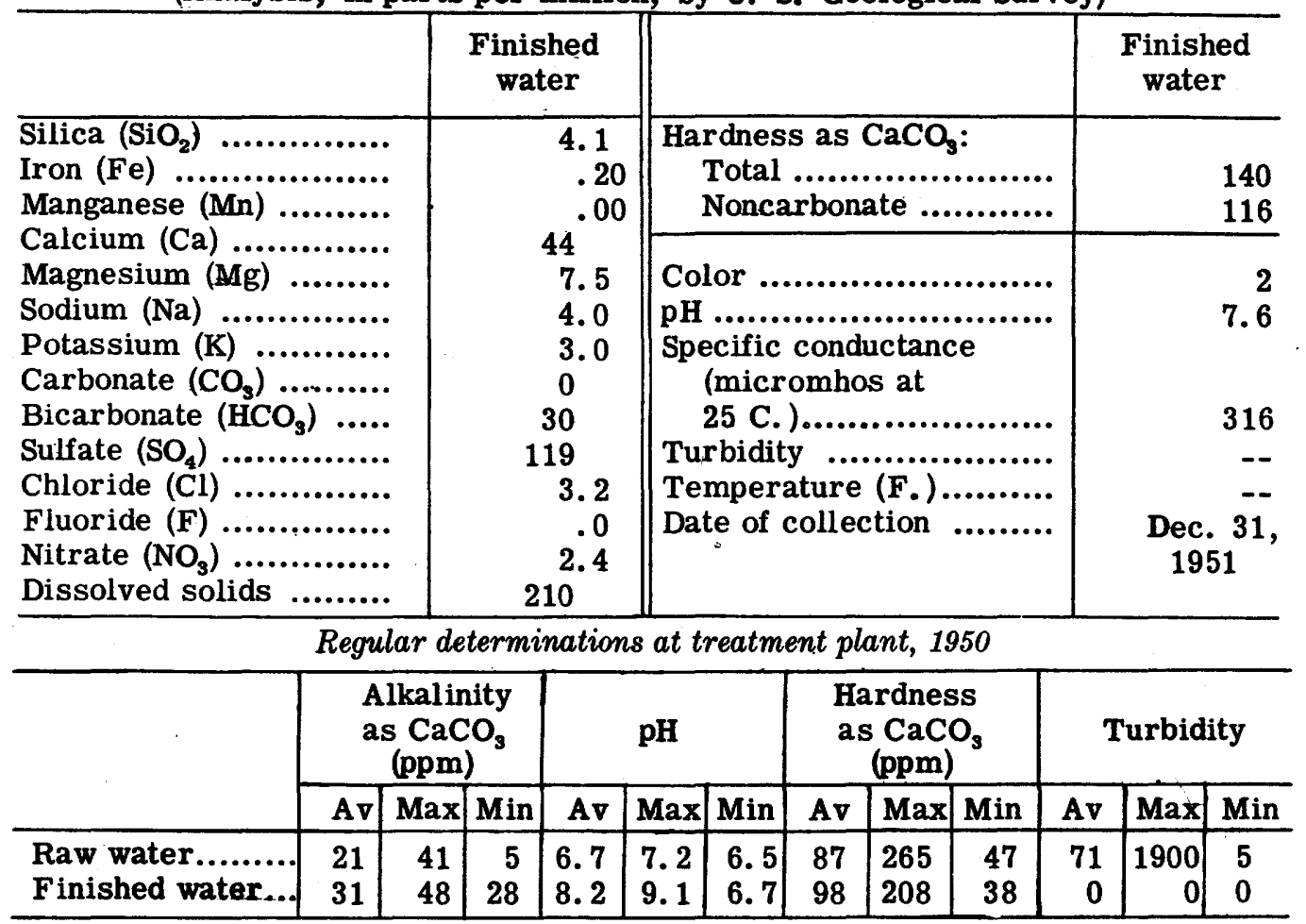




\section{ELKINS \\ (Population, 9, 121)}

Ownership: Municipal; supplies also about 500 people outside the city limits.

Total population supplied, about 9,600 .

Source: Tygart River.

Treatment: Prechlorination, coagulation with alum and lime, activated carbon at times, sedimentation, rapid sand filtration, postchlorination, and final adjustment of $\mathrm{pH}$ with lime.

Rated capacity of treatment plant: 2,000,000 gpd.

Raw-water storage: None.

Finished-water storage: 1, 350, 000 gal.

\section{ANALYSIS}

(Analysis, in parts per million, by U. S. Geological Survey)

\begin{tabular}{|c|c|c|c|}
\hline & $\begin{array}{c}\text { Finished } \\
\text { water }\end{array}$ & & $\begin{array}{c}\text { Finished } \\
\text { water }\end{array}$ \\
\hline 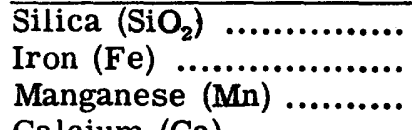 & $\begin{array}{r}3.2 \\
.03 \\
.00\end{array}$ & $\begin{array}{l}\text { Hardness as } \mathrm{CaCO}_{3}: \\
\text { Total ..................... } \\
\text { Noncarbonate ........... }\end{array}$ & $\begin{array}{l}45 \\
19 \\
\end{array}$ \\
\hline 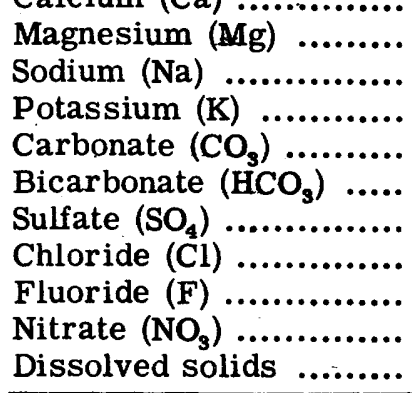 & $\begin{array}{r}14 \\
2.2 \\
1.5 \\
1.2 \\
0 \\
30 \\
22 \\
2.8 \\
.1 \\
64\end{array}$ & 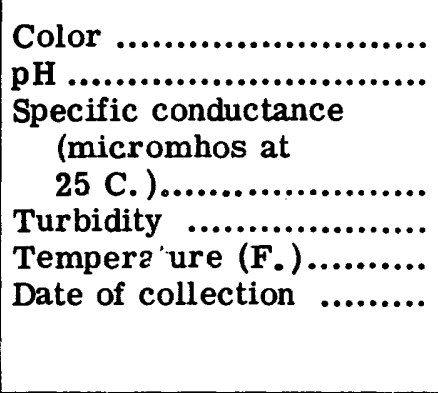 & $\begin{array}{c}108 \\
-- \\
-- \\
\text { Nov. } 23, \\
1951\end{array}$ \\
\hline
\end{tabular}

Regular determinations at treatment plant, 1950

\begin{tabular}{|c|c|c|c|c|c|c|c|c|c|c|c|c|}
\hline & \multicolumn{3}{|c|}{$\begin{array}{c}\text { Alkalinity } \\
\text { as } \mathrm{CaCO} \\
\text { (ppm) }\end{array}$} & \multicolumn{3}{|c|}{ pH } & \multicolumn{3}{|c|}{$\begin{array}{c}\text { Hardness } \\
\text { as } \mathrm{CaCO} \\
(\mathrm{ppm})\end{array}$} & \multicolumn{3}{|c|}{ Turbidity } \\
\hline & Av & $\operatorname{Max}$ & Min & Av & Max & $\operatorname{Min}$ & Av & $\operatorname{Max}$ & Min & Av & $\operatorname{Max}$ & Min \\
\hline $\begin{array}{l}\text { Raw water......... } \\
\text { Finished water... }\end{array}$ & $\begin{array}{l}20 \\
29\end{array}$ & \begin{tabular}{|l|}
24 \\
30
\end{tabular} & $\begin{array}{l}14 \\
26\end{array}$ & $\begin{array}{l}7.1 \\
8.2\end{array}$ & \begin{tabular}{|l|}
7.3 \\
8.3
\end{tabular} & $\begin{array}{l}6.8 \\
8.2\end{array}$ & -- & \begin{tabular}{|l|}
-- \\
--
\end{tabular} & - & $\overline{--}$ & $\begin{array}{l}-- \\
--\end{array}$ & -- \\
\hline
\end{tabular}


FAIRMONT

(Population, 29, 346)

Ownership: Municipal; supplies also Barracirville, Rivesville, and about 2,000 people outside the city limits. Total population supplied, about 33, 700 .

Source: Tygart River.

Treatment: Coagulation with alum and soda ash, prechlorination, sedimentation, rapid sand filtration, postchlorination, and addition of Calgon for corrosion control.

Rated capacity of treatment plant: $6,000,000 \mathrm{gpd}$.

Raw-water storage: $17,000,000$ gal.

Finished-water storage: 3, 500, 000 gal.

\section{ANALYSIS}

(Analysis, in parts per million, by U. S. Geological Survey)

\begin{tabular}{|c|c|c|c|}
\hline & $\begin{array}{c}\text { Finished } \\
\text { water }\end{array}$ & & $\begin{array}{c}\text { Finished } \\
\text { water }\end{array}$ \\
\hline \multirow{2}{*}{ 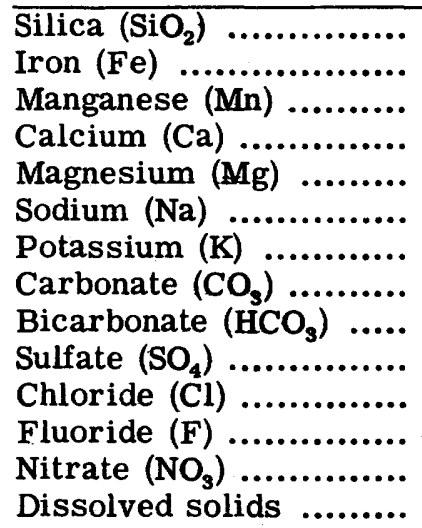 } & \multirow{2}{*}{$\begin{array}{c}3.9 \\
.20 \\
.14 \\
7.2 \\
1.2 \\
7.9 \\
2.0 \\
0 \\
13 \\
27 \\
1.5 \\
.0 \\
1.9 \\
58\end{array}$} & $\begin{array}{l}\text { Hardness as } \mathrm{CaCO}_{3}: \\
\quad \text { Total ....................... } \\
\text { Noncarbonate ........... }\end{array}$ & $\begin{array}{l}23 \\
12 \\
\end{array}$ \\
\hline & & 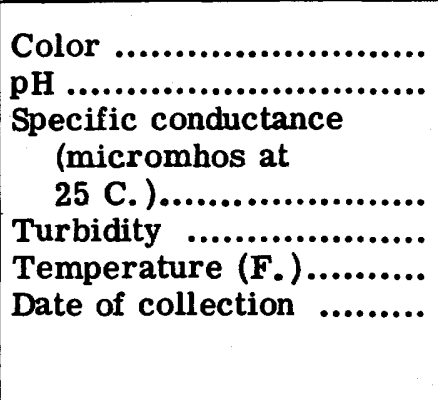 & $\begin{array}{r}96.6 \\
-- \\
-- \\
\text { Dec. } 27, \\
1951\end{array}$ \\
\hline
\end{tabular}

Regular determinations at treatment plant, 1950

\begin{tabular}{|c|c|c|c|c|c|c|c|c|c|c|c|c|}
\hline & \multicolumn{3}{|c|}{$\begin{array}{c}\text { Alkalinity } \\
\text { as } \mathrm{CaCO} \\
\text { (ppm) }\end{array}$} & \multicolumn{3}{|c|}{$\mathrm{pH}$} & \multicolumn{3}{|c|}{$\begin{array}{c}\text { Hardness } \\
\text { as } \mathrm{CaCO}_{3} \\
(\mathrm{ppm})\end{array}$} & \multicolumn{3}{|c|}{ Turbidity } \\
\hline & Av & Max & Min & Av & Max & Min & Av & Max & Min & Av & $\operatorname{Max}$ & Min \\
\hline $\begin{array}{l}\text { Raw water.......... } \\
\text { Finished water... }\end{array}$ & $\begin{array}{l}1.7 \\
9.9\end{array}$ & $\begin{array}{r}3.0 \\
24\end{array}$ & \begin{tabular}{|l|}
$\frac{a}{6} 18$ \\
6.0
\end{tabular} & $\begin{array}{l}6.0 \\
7.1\end{array}$ & \begin{tabular}{|l|}
6.6 \\
8.5
\end{tabular} & $\begin{array}{l}4.9 \\
6.9\end{array}$ & $\begin{array}{l}30 \\
30\end{array}$ & $\begin{array}{l}80 \\
78\end{array}$ & $\begin{array}{l}18 \\
18\end{array}$ & $\begin{array}{r}27 \\
0\end{array}$ & $\begin{array}{r}160 \\
0\end{array}$ & $\begin{array}{l}4 \\
0\end{array}$ \\
\hline
\end{tabular}

${ }^{\text {a }}$ Acidic. 


\section{HUNTINGTON}

(Population, 86, 353)

Ownership: Huntington Water Corporation; supplies also Barboursville, Chesapeake (Ohio), and about 30,000 people outside the city limits. Total population supplied, about 120,000 .

Souree: Ohio River.

Treatiment: Aeration, coagulation with alum and lime, activated carbon, sedimentation, rapid sand filtration, adjustment of $\mathrm{pH}$ with lime, and chlorination.

Rated capacity of treatment plant: 15,000,000 gpd.

Raw-water storage: None.

Finished-water storage: 2, 250,000 gal.

\section{ANALYSES}

(Analyses, in parts per million, by U. S. Geological Survey)

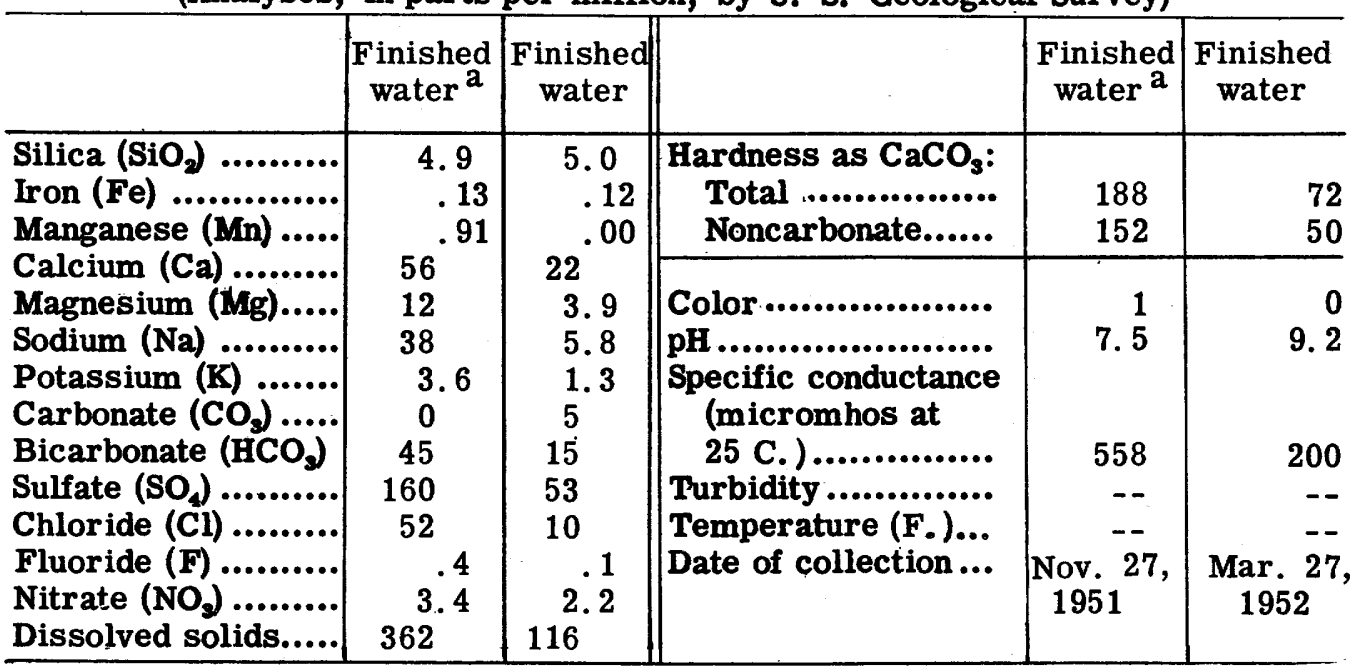

Regular determinations at treatment plant, 1950

\begin{tabular}{|c|c|c|c|c|c|c|c|c|c|c|c|c|}
\hline & \multicolumn{3}{|c|}{$\begin{array}{l}\text { Alkalinity } \\
\text { as } \mathrm{CaCO}_{3} \\
\text { (ppm) }\end{array}$} & \multicolumn{3}{|c|}{ pH } & \multicolumn{3}{|c|}{$\begin{array}{c}\text { Hardness } \\
\text { as CaCO } \\
\text { (ppm) }\end{array}$} & \multicolumn{3}{|c|}{ Turbidity } \\
\hline & Av & Max & Min & Av & Max & Min & Av & Max & Min & Av & Max & Min \\
\hline $\begin{array}{l}\text { Raw water.......... } \\
\text { Finished water... }\end{array}$ & $\begin{array}{l}25 \\
30\end{array}$ & $\begin{array}{l}53 \\
50\end{array}$ & $\begin{array}{l}13 \\
20 \\
\end{array}$ & $\begin{array}{l}7.1 \\
8.4\end{array}$ & $\begin{array}{l}7.4 \\
8.4\end{array}$ & $\begin{array}{l}6.5 \\
7.9\end{array}$ & $\overline{70}$ & $\overline{134}$ & $\overline{28}$ & -- & $\begin{array}{r}600 \\
0\end{array}$ & $\begin{array}{r}15 \\
0\end{array}$ \\
\hline
\end{tabular}

a River at low stage at time of collection of sample. 


\section{MARTINSBURG}

(Population, 15, €21)

Ownership: Municipal; supplies also about 100 people outside the city limits.

Total population supplied, about 15,700.

Source: Limestone spring. Auxiliary supply from spring-fed limestone quarry. Treatment: Chlorination.

Raw-water storage: 200,000, 000 gal (auxiliary supply).

Finished-water storage: $3,750,000$ gal.

\section{ANALYSIS}

(Analysis, in parts per million, by U. S. Geological Survey)

\begin{tabular}{|c|c|c|c|}
\hline & $\begin{array}{l}\text { Finished } \\
\text { water }\end{array}$ & & $\begin{array}{c}\text { Finished } \\
\text { water }\end{array}$ \\
\hline 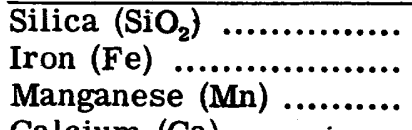 & $\begin{array}{rr}11 & \\
.19 \\
.00\end{array}$ & $\begin{array}{l}\text { Hardness as } \mathrm{CaCO}_{3}: \\
\text { Total } \ldots . . . . . . . . \ldots \ldots \ldots \ldots \\
\text { Noncarbonate .............. }\end{array}$ & $\begin{array}{r}264 \\
31 \\
\end{array}$ \\
\hline 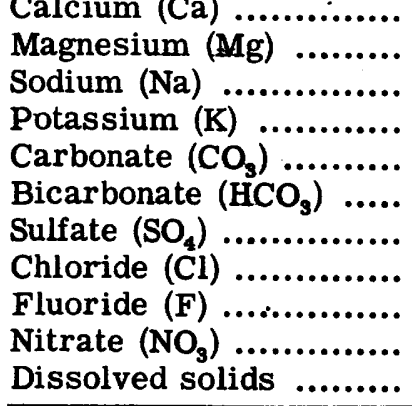 & $\begin{array}{c}80 \\
16 \\
1.6 \\
1.8 \\
0 \\
286 \\
18 \\
3.5 \\
14 \\
279\end{array}$ & 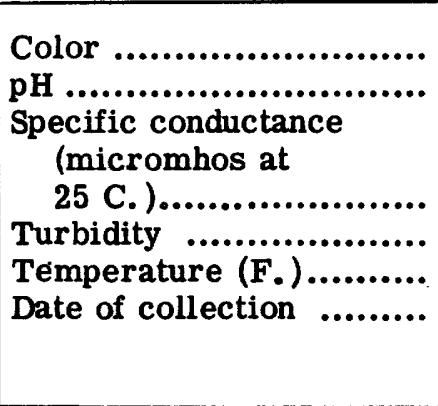 & Feb. 27 , \\
\hline
\end{tabular}




\section{MORGANTOWN}

(Population, 25, 525)

Ownership: Municipal; supplies also Star City, Westover, and about 8,000 people outside the city limits. Total population supplied, about 39,000.

Source: Monongahela River, 75 percent of supply; Tibbs Run, impounded, 25 percent of supply.

Treatment: Monongahela River supply: coagulation with alum and lime, activated carbon, sedimentation, rapid sand filtration, zeolite softening, and chlorination. Tibbs Run supply: addition of lime, and chlorination.

Rated capacity of treatment plant: $3,800,000$ gpd.

Raw-water storage: $35,000,000$ gal.

Finished-water stor age: 2,600,000 gal.

\section{ANALYSES}

(Analyses, in parts per million, by U. S. Geological Survey)

\begin{tabular}{|c|c|c|c|}
\hline & $\begin{array}{l}\text { Finished water } \\
\text { (Monongahela } \\
\text { River) }\end{array}$ & $\begin{array}{l}\text { Finished water } \\
\text { (Tibbs Run, } \\
\text { impounded) }\end{array}$ & $\begin{array}{c}\text { Filter effluent } \\
\text { (Monongahela } \\
\text { River) }\end{array}$ \\
\hline Silica $\left(\mathrm{SiO}_{2}\right) \ldots . .$. & 3.7 & 4.1 & 3.7 \\
\hline Iron $(\mathrm{Fe})$........... & .12 & .13 & .12 \\
\hline Manganese (Mn) ....... & .00 & .00 & .04 \\
\hline Calcium (Ca) $\ldots . . . . . . . . . . . . . . . . . .$. & 30 & 7.0 & 41 \\
\hline Magnesium (Mg)..................... & 4.9 & 1.2 & 7.1 \\
\hline Sodium (Na)........... & 33 & 1.2 & 10 \\
\hline Fotassium (K) $\ldots \ldots \ldots \ldots \ldots \ldots \ldots$ & 2.0 & 1.7 & 2.2 \\
\hline Carbonate $\left(\mathrm{CO}_{3}\right) \ldots \ldots \ldots \ldots \ldots \ldots$ & 0 & 0 & 0 \\
\hline Bicarbonate $\left(\mathrm{HCO}_{3}\right) \ldots \ldots \ldots \ldots \ldots . . . .$. & 19 & 13 & 17 \\
\hline 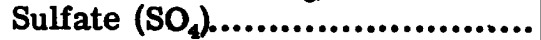 & 140 & 13 & 135 \\
\hline Chloride (Cl) ...................... & 7.5 & 2.5 & 3.2 \\
\hline Fluoride $(F)$.................... & .1 & .1 & .1 \\
\hline Nitrate $\left(\mathrm{NO}_{3}\right), \ldots \ldots \ldots \ldots \ldots \ldots \ldots . . . . . . . . . .$. & 2.4 & .4 & 2.4 \\
\hline Dissolved solids ................... & 237 & 39 & 222 \\
\hline Hardness as $\mathrm{CaCO}_{3}:$ & 04 & 92 & 139 \\
\hline Noncarbonate .......................... & 79 & 12 & 118 \\
\hline Color.. & & $\mathbf{5}$ & \\
\hline pH ................... & 8.5 & 7.2 & 8.1 \\
\hline Specific conductance & & & \\
\hline (micromhos at 25 C. )... & 371 & 60.1 & 339 \\
\hline Turbidity.................... & -- & -- & -- \\
\hline Temperature (F.)... & & -- & -- \\
\hline Date of collection .... & Jan. 1, 1952 & Jan. 1, 1952 & Jan. 1, 1952 \\
\hline
\end{tabular}

Regular determinations at treatment plant, 1951

\begin{tabular}{|c|c|c|c|c|c|c|c|c|c|c|c|c|}
\hline & \multicolumn{3}{|c|}{$\begin{array}{l}\text { Alkalinity } \\
\text { as CaCO } \\
\text { (ppm) }\end{array}$} & \multicolumn{3}{|c|}{ pH } & \multicolumn{3}{|c|}{$\begin{array}{l}\text { Hardness } \\
\text { as } \mathrm{CaCO}_{3} \\
(\mathrm{ppm})\end{array}$} & \multicolumn{3}{|c|}{ Turbidity } \\
\hline & Av & $\operatorname{Max}$ & Min & Av & Max & Min & Av & $\operatorname{Max}$ & Min & Av & $\operatorname{Max}$ & Min \\
\hline $\begin{array}{l}\text { Raw water.......... } \\
\text { Finished water... } \\
\text { Finished water... }\end{array}$ & $\begin{array}{l}-- \\
12 \\
13\end{array}$ & \begin{tabular}{r|}
6 \\
16 \\
20 \\
\end{tabular} & $\begin{array}{r}-13 \\
10 \\
9 \\
\end{array}$ & $\begin{array}{l}-- \\
9.0 \\
9.0\end{array}$ & \begin{tabular}{|l|}
-- \\
9.4 \\
9.4 \\
\end{tabular} & $\begin{array}{l}--- \\
8.8 \\
8.8\end{array}$ & \begin{tabular}{|l|}
92 \\
64 \\
28 \\
\end{tabular} & \begin{tabular}{|r|}
205 \\
166 \\
34
\end{tabular} & $\begin{array}{l}42 \\
42 \\
24\end{array}$ & $\begin{array}{r}40 \\
0 \\
0\end{array}$ & $\begin{array}{r}400 \\
5 \\
0\end{array}$ & $\begin{array}{l}5 \\
0 \\
0\end{array}$ \\
\hline
\end{tabular}

${ }_{b}^{a}$ Monongahela River.

b Tibbs Run. 


\section{MOUNDSVILLE}

(Population, 14, 772)

Ownership: Municipal.

Source: 3 wells (4 to 6), 69, 69, and $70 \mathrm{ft}$ deep, yield reported to be 400,400 , and $725 \mathrm{gpm}$. Emergency supply from Well 3.

Treatment: Chlorination. Emergency supply treated with polyphosphate (Calgon) for manganese control.

Raw-water storage: None.

Finished-water storage: 2, 000, 000 gal.

\section{ANALYSIS}

(Analysis, in parts per million, by U. S. Geological Survey)

\begin{tabular}{|c|c|c|c|}
\hline & $\begin{array}{r}\text { Finished } \\
\text { water a }\end{array}$ & & $\begin{array}{r}\text { Finished } \\
\text { water a }\end{array}$ \\
\hline \multirow{2}{*}{ 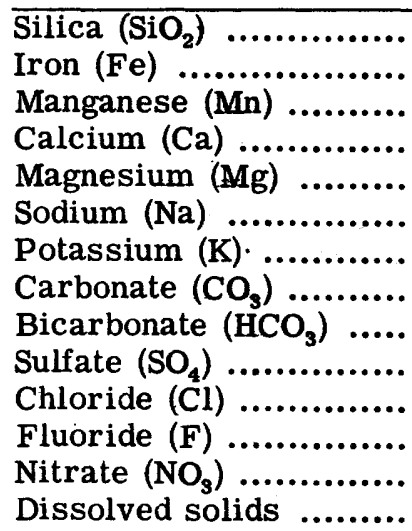 } & \multirow{2}{*}{$\begin{array}{l}{ }^{11} \\
.12 \\
.02 \\
87 \\
14 \\
23 \\
2.6 \\
0 \\
95 \\
213 \\
21 \\
.1 \\
2.2 \\
445\end{array}$} & 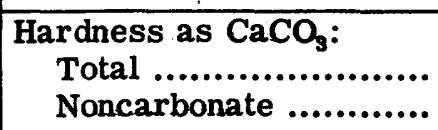 & $\begin{array}{l}274 \\
197\end{array}$ \\
\hline & & 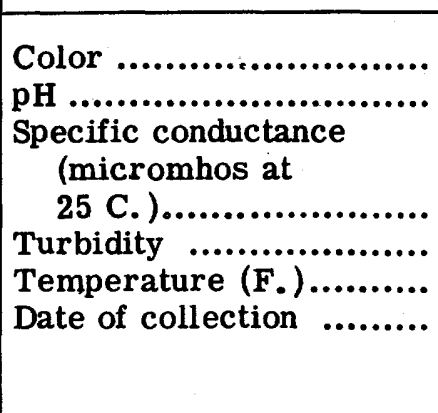 & $\begin{array}{r}611 \\
-- \\
\text { Dec. } 11, \\
1951\end{array}$ \\
\hline
\end{tabular}

Regular determinations at treatment plant, 1950

\begin{tabular}{|c|c|c|c|c|c|c|c|c|c|c|c|c|}
\hline & \multicolumn{3}{|c|}{$\begin{array}{c}\text { Alkalinity } \\
\text { as } \mathrm{CaCO} \mathrm{O}_{3} \\
\text { (ppm) }\end{array}$} & \multicolumn{3}{|c|}{$\mathrm{pH}$} & \multicolumn{3}{|c|}{$\begin{array}{c}\text { Hardness } \\
\text { as } \mathrm{CaCO}_{3} \\
\text { (ppm) }\end{array}$} & \multicolumn{3}{|c|}{ Turbidity } \\
\hline & Av & Max & Min & Av & $\operatorname{Max}$ & Min & Av & Max & Min & Av & $\operatorname{Max}$ & Min \\
\hline $\begin{array}{l}\text { Raw water......... } \\
\text { Finished water... }\end{array}$ & $\overline{82}$ & --- & $\overline{66}$ & $7 .-2$ & $7 .--$ & 7.0 & $\overline{--}$ & 276 & $\overline{168}$ & -- & -- & $\begin{array}{l}-- \\
--\end{array}$ \\
\hline
\end{tabular}

${ }^{\mathrm{a}}$ Composite, wells 5 and 6. 
NITRO

(Population, 3, 314)

Ownership: West Virginia Water Service Company; supplies also Dunbar, and about 11,500 people outside the city limits. The system is interconnected with Belle, Charleston, and St. Albans. Total population supplied, about 22,800 .

Source: Kinawha River.

Treatment: Aeration, prechlorination, coagulation with alum, activated carbon, sedimentation, rapid sand filtration, adjustment of $\mathrm{pH}$ with lime, and postchlorination.

Rated capacity of treatment plant: $36,000,000 \mathrm{gpd}$

Raw-water storage: None.

Finished-water storage: 1, 800, 000 gal.

\section{ANALYSIS}

(Analysis, in parts per million, by U. S. Geological Survey)

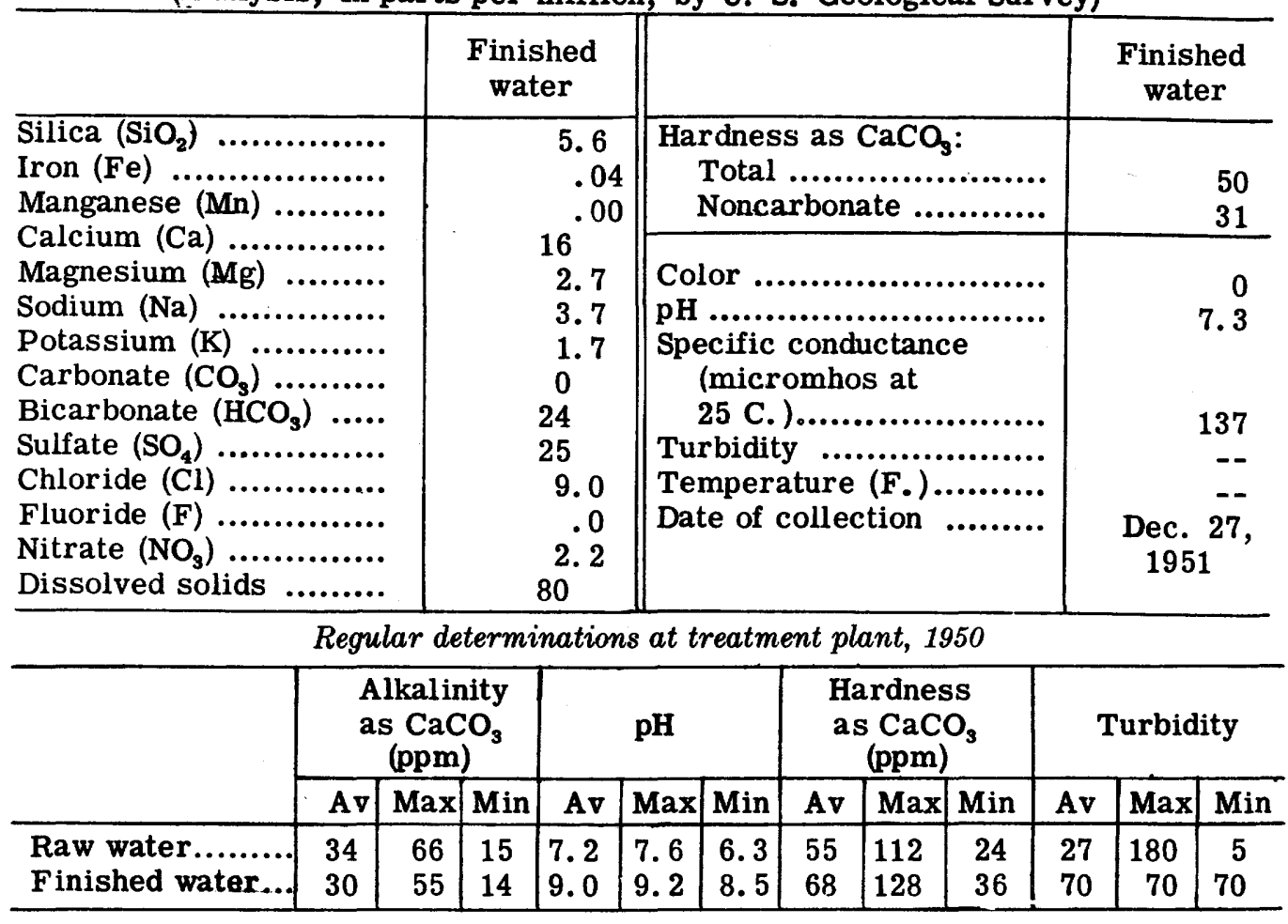




\section{PARKERSBURG}

(Population, 40,492)

Ownership: Municipal; supplies also about 5,000 people outside the city limits.

Total population supplied, about 45,500 .

Source: 3 wells (Ranney collectors 1 to 3 ), 57, 56, and $54 \mathrm{ft}$ deep, yield reported to be $2,500,2,000$, and 2,500 gpm. Emergency supply from Ohio River.

Treatment: Prechlorination, aeration, coagulation with lime, sedimentation, rapid sand filtration, addition of polyphosphate (Calgon), and postchlorination.

Rated capacity of treatment plant: 8,000,000 gpd.

Raw-water storage: None.

Finished-water storage: 7, 500,000 gal.

\section{ANALYSIS}

(Analysis, in parts per million, by U. S. Geological Survey)

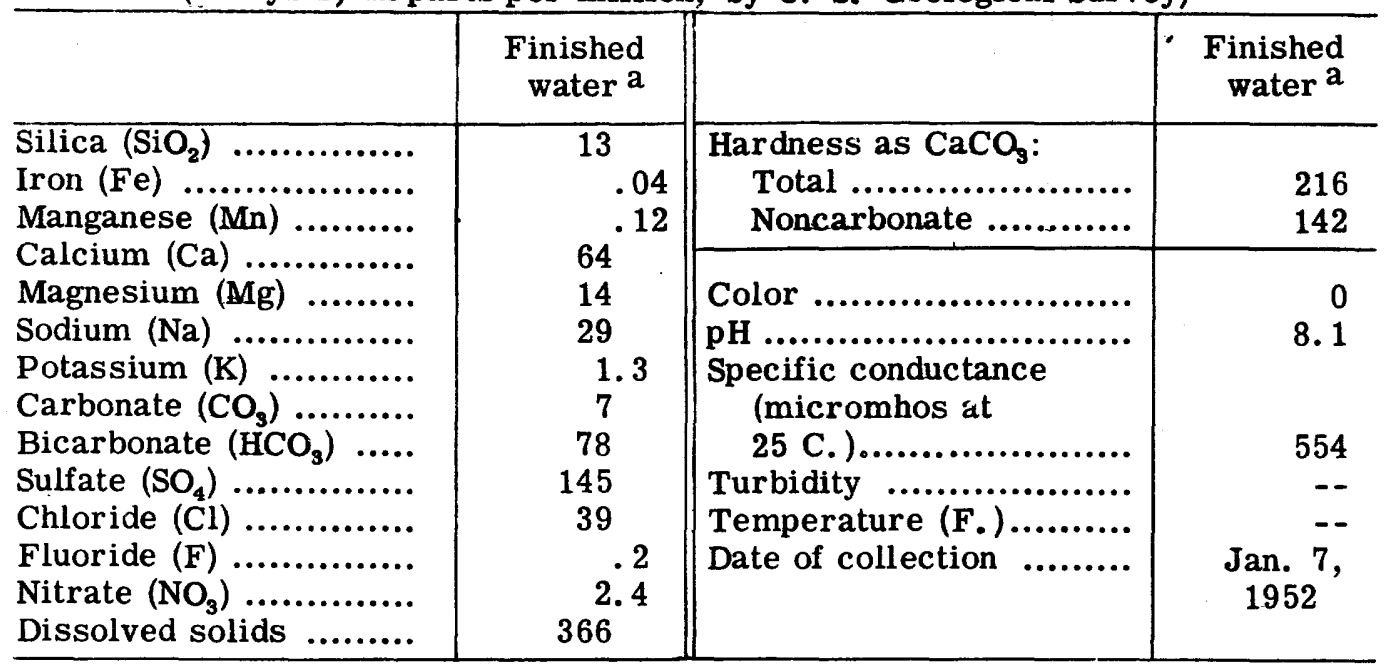

Regular determinations at treatment plant, 1950

\begin{tabular}{l|c|c|c|c|c|c|c|c|c|c|c|c}
\hline & \multicolumn{3}{|c|}{$\begin{array}{c}\text { Alkalinity } \\
\text { as CaCO } \\
\text { (ppm) }\end{array}$} & \multicolumn{3}{c|}{ pH } & \multicolumn{3}{c|}{$\begin{array}{c}\text { Hardness } \\
\text { as CaCO } \\
\text { (ppm) }\end{array}$} & \multicolumn{3}{c}{ Turbidity } \\
\cline { 2 - 14 } & Av & Max & Min & Av & Max & Min & Av & Max & Min & Av & Max & Min \\
\hline Raw water......... & 71 & 75 & 69 & 6.6 & 6.7 & 6.5 & 159 & 173 & 151 & -- & -- & -- \\
Finished water... & 82 & 86 & 81 & 8.4 & 8.6 & 8.2 & 167 & 178 & 157 & -- & -- & -- \\
\hline
\end{tabular}

${ }^{\mathrm{a}}$ Composite, collectors 1 and 3 . 


\section{ST. ALBANS}

(Population, 9, 870)

Ownership: West Virginia Water Service Company; supplies also about 8,500 people outside St. Albans. The system is interconnected with Belle, Charleston, and Nitro. Total population supplied, about 18,400 .

Source: Coal River.

Treatment: Prechlorination, coagulation with alum and lime, sedimentation, rapid sand filtration, adjustment of $\mathrm{pH}$ with lime, postchlorination. Aeration and activated carbon at times.

Rated capacity of treatment plant: 1,600,000 gpd.

Raw-water storage: None.

Finished-water storage: 300,000 gal.

\section{ANALYSIS}

(Analysis, in parts per million, by U. S. Geological Survey)

\begin{tabular}{|c|c|c|c|}
\hline & $\begin{array}{c}\text { Finished } \\
\text { water }\end{array}$ & & $\begin{array}{l}\text { Finished } \\
\text { water }\end{array}$ \\
\hline $\begin{array}{l}\text { Silica }\left(\mathrm{SiO}_{2}\right) \ldots \ldots \ldots \ldots \ldots \ldots \\
\text { Iron }(\mathrm{Fe}) \quad \ldots \ldots \ldots \ldots \ldots \ldots . . \\
\text { Manganese }(\mathrm{Mn}) \ldots \ldots \ldots \ldots\end{array}$ & $\begin{array}{l}6.6 \\
.05 \\
.00\end{array}$ & $\begin{array}{l}\mathrm{Hardness} \text { as } \mathrm{CaCO}_{3}: \\
\text { Total ...................... } \\
\text { Noncarbonate .......... }\end{array}$ & $\begin{array}{l}51 \\
37\end{array}$ \\
\hline 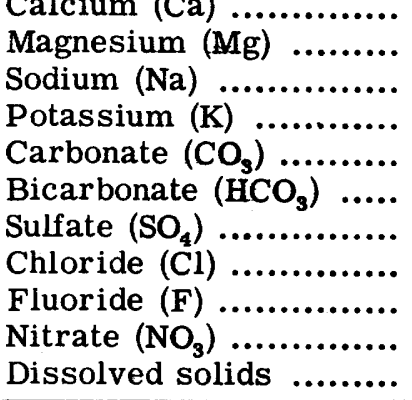 & $\begin{array}{r}14 \\
3.9 \\
4.5 \\
2.6 \\
0 \\
17 \\
40 \\
4.8 \\
.0 \\
1.9 \\
86\end{array}$ & 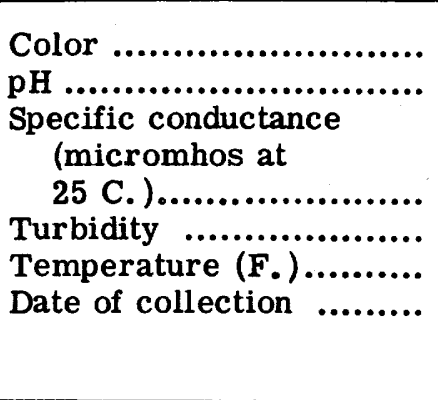 & $\begin{array}{r}147 \\
\text { Dec. } 27, \\
1951\end{array}$ \\
\hline
\end{tabular}

Regular determinations at treatment plant, 1950

\begin{tabular}{|c|c|c|c|c|c|c|c|c|c|c|c|c|}
\hline & \multicolumn{3}{|c|}{$\begin{array}{l}\text { Alkalinity } \\
\text { as } \mathrm{CaCO}_{3} \\
\text { (ppm) }\end{array}$} & \multicolumn{3}{|c|}{ pH } & \multicolumn{3}{|c|}{$\begin{array}{c}\text { Hardness } \\
\text { as CaCO } \\
\text { (ppm) }\end{array}$} & \multicolumn{3}{|c|}{ Turbidity } \\
\hline & $\mathrm{Av}$ & $\operatorname{Max}$ & Min & $A \nabla$ & $\operatorname{Max}$ & Min & $\mathbf{A v}$ & $\operatorname{Max}$ & Min & Av & Max & Min \\
\hline $\begin{array}{l}\text { Raw water.......... } \\
\text { Finished water... }\end{array}$ & $\begin{array}{l}20 \\
25\end{array}$ & $\begin{array}{l}58 \\
62\end{array}$ & $\begin{array}{l}10 \\
15\end{array}$ & $\begin{array}{l}6.8 \\
9.0\end{array}$ & $\begin{array}{l}7.3 \\
9.4\end{array}$ & $\begin{array}{l}6.3 \\
8.2\end{array}$ & $\begin{array}{l}50 \\
62\end{array}$ & $\begin{array}{l}100 \\
110\end{array}$ & $\begin{array}{l}24 \\
32\end{array}$ & $\begin{array}{r}61 \\
0\end{array}$ & $\begin{array}{r}1200 \\
0\end{array}$ & $\begin{array}{r}.8 \\
0\end{array}$ \\
\hline
\end{tabular}

SOUTH CHARLESTON

(Population, 16, 686)

Ownership: West Virginia Water Service Company.

Source: From Nitro and Charleston.

Treatment: (See Nitro and Charleston.) 


\section{WEIRTON \\ (Population, 24, 005)}

Ownership: Municipal.

Source: Ohio River.

Treatment: Aeration, coagulation with lime and ferrous sulfate at times, superchlorination, activated carbon at times, fluoridation with sodium silicofluoride, sedimentation, rapid sand filtration, and chlorine dioxide.

Rated capacity of treatment plant: 2, 000, $000 \mathrm{gpd}$.

Raw-water storage: None.

Finished-water storage: $1,720,000$ gal.

\section{ANALYSIS}

(Analysis, in parts per million, by U. S. Geological Survey)

\begin{tabular}{|c|c|c|c|}
\hline & $\begin{array}{c}\text { Finished } \\
\text { water }\end{array}$ & & $\begin{array}{l}\text { Finished } \\
\text { water }\end{array}$ \\
\hline $\begin{array}{l}\text { Silica }\left(\mathrm{SiO}_{2}\right) \quad \ldots \ldots \ldots \ldots \ldots \ldots \\
\text { Iron }(\mathrm{Fe}) \quad \ldots \ldots \ldots \ldots \ldots \ldots \\
\text { Manganese }(\mathrm{Mn}) \ldots \ldots \ldots \ldots\end{array}$ & $\begin{array}{r}4.4 \\
.21 \\
.00\end{array}$ & $\begin{array}{l}\text { Hardness as } \mathrm{CaCO}_{3}: \\
\text { Total ....................... } \\
\text { Noncarbonate ........... }\end{array}$ & $\begin{array}{r}100 \\
81 \\
\end{array}$ \\
\hline 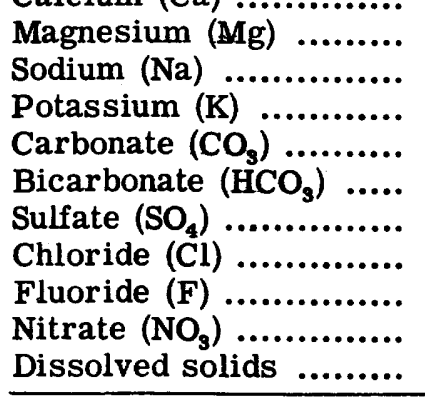 & $\begin{array}{l}4.4 \\
14 \\
2.6 \\
0 \\
24 \\
77 \\
22 \\
1.6 \\
2.0 \\
180 \\
\end{array}$ & 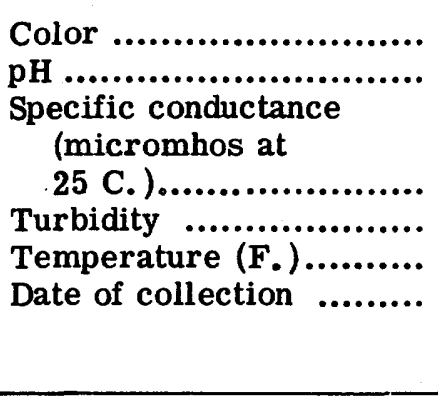 & $\begin{array}{r}289 \\
-- \\
-- \\
\text { Dec. } 12, \\
1951\end{array}$ \\
\hline
\end{tabular}

Regular determinations at treatment plant, 1950

\begin{tabular}{|c|c|c|c|c|c|c|c|c|c|c|c|c|}
\hline & \multicolumn{3}{|c|}{$\begin{array}{l}\text { Alkalinity } \\
\text { as CaCO } \\
\text { (ppm) }\end{array}$} & \multicolumn{3}{|c|}{ pH } & \multicolumn{3}{|c|}{$\begin{array}{c}\text { Hardness } \\
\text { as CaCO, } \\
\text { (ppm) }\end{array}$} & \multicolumn{3}{|c|}{ Turbidity } \\
\hline & Av & $\operatorname{Max}$ & Min & $\mathbf{A v}$ & $\operatorname{Max}$ & Min & Av & $\operatorname{Max}$ & Min & $A v$ & $\operatorname{Max}$ & Min \\
\hline $\begin{array}{l}\text { Raw water......... } \\
\text { Finished water... }\end{array}$ & $\begin{array}{r}8 \\
26\end{array}$ & $\begin{array}{l}22 \\
32\end{array}$ & $\begin{array}{r}0 \\
12\end{array}$ & $\begin{array}{l}6.7 \\
9.5\end{array}$ & $\begin{array}{l}7.2 \\
9.6\end{array}$ & $\begin{array}{l}5.3 \\
9.2\end{array}$ & $\begin{array}{l}112 \\
125\end{array}$ & $\begin{array}{l}205 \\
188\end{array}$ & $\begin{array}{l}60 \\
68\end{array}$ & $\begin{array}{r}120 \\
0\end{array}$ & $\begin{array}{r}1100 \\
0\end{array}$ & $\begin{array}{r}30 \\
0\end{array}$ \\
\hline
\end{tabular}


WHEELING

(Population, 58, 891)

Ownership: Municipal; supplies also Bethlehem, Triadelphia, and about 3,600 people outside the city limits. Total population supplied, about 64,400 .

Source: Ohio River.

Treatment: Aeration, superchlorination, coagulation with alum and ferrous sulfate, sedimentation, rapid sand filtration, chlorination, chlorine dioxide, adjustment of $\mathrm{pH}$ with caustic soda, and flouridation with sodium silicofluoride.

Rated capacity of treatment plant: 20,000,000 gpd.

Raw-water storage: None.

Finished-water storage: $11,500,000$ gal.

ANALYSIS

(Analysis, in parts per million, by U. S. Geological Survey)

\begin{tabular}{|c|c|c|c|}
\hline & $\begin{array}{c}\text { Finished } \\
\text { water }\end{array}$ & & $\begin{array}{c}\text { Finished } \\
\text { water }\end{array}$ \\
\hline $\begin{array}{l}\text { Silica }\left(\mathrm{SiO}_{2}\right) \quad \ldots \ldots \ldots \ldots \ldots \\
\text { Iron }(\mathrm{Fe}) \quad \ldots \ldots \ldots \ldots \ldots \ldots \\
\text { Manganese }(\mathbf{M n}) \ldots \ldots \ldots \ldots\end{array}$ & $\begin{array}{r}5.2 \\
.06 \\
.00\end{array}$ & $\begin{array}{l}\text { Hardness as } \mathrm{CaCO}_{3}: \\
\text { Total ...................... } \\
\text { Noncarbonate .......... }\end{array}$ & $\begin{array}{l}128 \\
105\end{array}$ \\
\hline 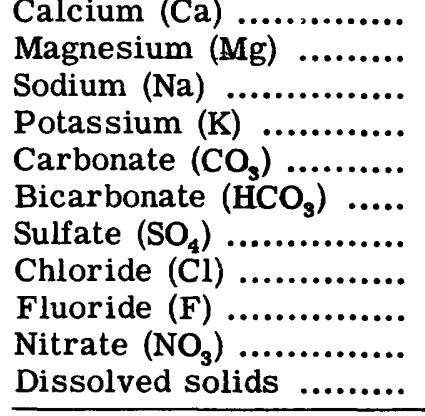 & $\begin{array}{c}40 \\
7.0 \\
14 \\
2.4 \\
0 \\
29 \\
104 \\
22 \\
.6 \\
1.9 \\
224 \\
\end{array}$ & 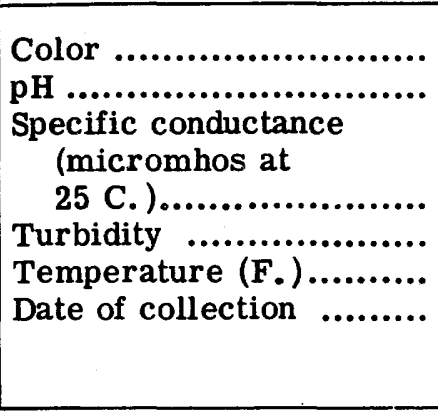 & $\begin{array}{r}345 \\
-- \\
-- \\
\text { Dec. } 11 \\
1951\end{array}$ \\
\hline
\end{tabular}

Regular determinations at treatment plant, 1950

\begin{tabular}{|c|c|c|c|c|c|c|c|c|c|c|c|c|}
\hline & \multicolumn{3}{|c|}{$\begin{array}{l}\text { Alkalinity } \\
\text { as CaCo } \\
\text { (ppm) }\end{array}$} & \multicolumn{3}{|c|}{$\mathrm{pH}$} & \multicolumn{3}{|c|}{$\begin{array}{l}\text { Hardness } \\
\text { as } \mathrm{CaCO} \\
(\mathrm{ppm})\end{array}$} & \multicolumn{3}{|c|}{ Turbidity } \\
\hline & Av & Max & Min & Av & $\operatorname{Max}$ & Min & Av & $\operatorname{Max}$ & Min & Av & $\operatorname{Max}$ & Min \\
\hline $\begin{array}{l}\text { Raw water.......... } \\
\text { Finished water... }\end{array}$ & \begin{tabular}{l|}
10 \\
22
\end{tabular} & \begin{tabular}{|l|}
24 \\
43 \\
\end{tabular} & \begin{tabular}{r|}
4 \\
14
\end{tabular} & $\begin{array}{l}6.6 \\
9.0\end{array}$ & $\begin{array}{l}7.5 \\
9.8 \\
9\end{array}$ & \begin{tabular}{|l|}
5.5 \\
8.1
\end{tabular} & $\begin{array}{l}111 \\
141\end{array}$ & $\begin{array}{l}192 \\
228\end{array}$ & $\begin{array}{l}52 \\
84 \\
\end{array}$ & $\begin{array}{r}80 \\
0\end{array}$ & $\begin{array}{r}800 \\
0 \\
\end{array}$ & $\begin{array}{r}20 \\
0 \\
\end{array}$ \\
\hline
\end{tabular}

